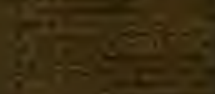


$59.82: 01$

FOR THE PEOPLE FOR EDVCATION FOR SCIENCE

\author{
LIBRARY \\ of
}

THE AMERICAN MUSEUM

of

NATURAL HISTORY 
if $O M E$ I C LA T URE

$$
\text { BIR DS } 59.82: 01
$$

George Robert Gray

\author{
( Unpublished book \\ about 1851)
}


homenclation of Birds $17 \cdot 1-324$.

G.R. Gray is us doubt the ant ion as . . $\$$

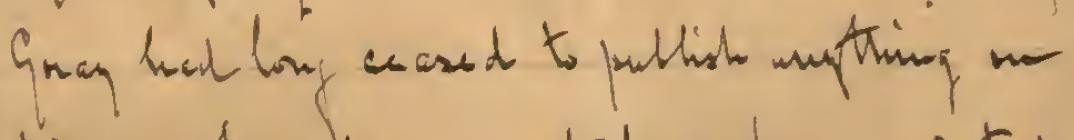
Lind when this mut Lav been printed

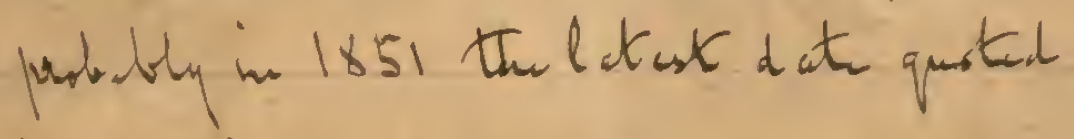
in it is p 289,318 \& 519 .

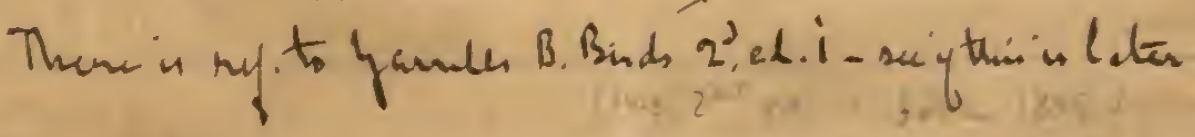

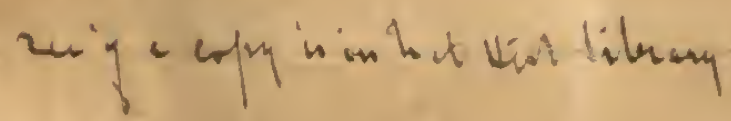

a van rare work mpateishio. 


\title{
NOMENCLATURE OF BIRDS.
}

\author{
$\frac{29-115926-70 \div-26}{0}$
}

K I N D о M, A N I M A L I A.

S U в IN g D о м, VER T E B R A T A.

\section{Class II. AVES. \\ Order I. ACCIPITRES.}

Accipitres, Linn. 1735; Mochr. 1752；Lath. 1790; Cur. 1895 $-1796$.

Dermatopodes, Mcehr. 1752.

Plumiperes, p. Schaff. 1774.

Fissipedes anisodactyles, aduncirostres, Schaff. 1774.

Retipedes, p. Scop. 1777 .

Rapaces, Scop. 1777 ; Temm. 1815; Ranz. 1823.

Raptatores, Illig. 1811.

Raptores, Vigors, 1826.

Raptrices, Macgill. 1840.

Gressores, p. G. accipitres, Sundev. 1835.

\section{Suborder I. ACCIPITRES DIURNI.}

Accipitres diurni, Vieill. 1816 ; Cuv. 1817.

Nudicolles-Plumicolles-Grallæ, p.-Brevirostres, Cur. 17991800.

Vulturini-Accipitrini, Illig. 1811.

Grallatores, p.-Uncirostres, p. Vieill. 1816.

Diurni, Ranz. 1823. 


\section{GYPAETIDÆ.}

Gypaëtidæ, Gr. 1842. Pr. B. 1850.

Vulturidæ, p. Sw. 1837. G. R. Gr. 1840-41-44. Pr. B. 1849 -50 .

\section{i. Gypaetine.}

Gypaëtinæ, Pr. B. 1831-38-49. G. R. Gr. 1840-41-44. Bl. 1849. Cass. 1849.

\section{Gypaetus.}

Gypaëtus, Storr. 1784.

Phene, Sav. 1809.

Gyptus, Dum. 1806.

1. Gypaetus barbatus.

B. M.

Vultur barbatus, $L . E d w . B . p l .106 . \quad$ V. barbarus et V. niger, Gm. V. aureus, Briss. Falco magnus, S. G. Gm. Gypaètus grandis, Storr. G. alpinus, G. castaneus et G. aureus, Daud. G. leucocephalus et G. melanocephalus, Mey. G. harbatus, Cuv. G. barbatus occidentalis, $S c h l$. G. nudipes et G. subalpinus, Brehm. G. meridionalis, Schl. G. altaicus, Gebl. Phene ussifraga, Sav. Gypaëtus barbatus, et G. hemachalanus, Hutt. Pl. col. 431. Rüpp. Syst. Uebers. Vog. N. Ost. Afr. t. 1. Gould, B. of Eur.pl. 4.

Mountains of Europe, Asia, N. E. Africa.

\section{VULTURIDA.}

Vulturidæ. Vig. 1825. Pr. B. 1831-38-49-50; Sw. 1831-37; G. R. Gr. 1840-41-44. Bl. 1849; Cass. 1849.

Vulturides, Sundev. 1835.

Sarcoramphidæ, Gr. 1848.

Falconidæ, p. Pr. B. 1831.

Cathartidæ, Gr. 1842.

Gypobieracidæ, Pr. B. 1850.

\section{i. Sarcoramphinde.}

Sarcoramphinæ, G. R. Gr. 1844. Bl. 1849. Cass. 1849. Vulturinæ, p. $\operatorname{Pr} . B .1838$.

Cathartinæ, G. R. Gr. 1840-41. Pr. B. $1849-50$. 


\section{Sarcoramphus.}

a. Sarcoramphus, Dum. 1806. Gypagus, Vieill. 1816. Cathartes, p. Ill. 1811. Zopilotes, Flem. 1822.

2. Sarcoramphus gryphus.

B. $M$ •

Vultur gryphus, $L$. $P l$. col. $133,408,494$. V. magellanicus' Shaw, Lev. Mus. pl. 1. V. condor, Shaw. Sarcoramphus gryphus, Goldf. S. cuntur, Dum. S. condor, Less. Gypagus gryphus, Vieill. Voy. de la Bonite, Ois, t. 2. Pr. B. Amer. Orn. iv. pl. 22. Humb. Obs. de Zool. t. 13.

S. America, (Andes), and S. W. of N. America.

3. Sarcoramphus papa.

B. M.

Vultur papa, L. $P l$. enl. 428. Cathartes pápa, Ill. Sarcoramphus papa, Dum. Gypagus papa, Vieill. Levaill. Ois. de l'Afr. t. 13. Spix, Av. Bras. ᄂ. 1. Edw. B. pl. 2.

S. America, (Mexico, Guiana, Brazil); S. of N. America.

b. Catharista, Vieill. 1816.

Cathartes, p. Ill. 1811.

4. Sarcoramphus californianus.

B. M.

Vultur californianus, Shaw, Nat. Misc. pl. 301. Cathartes californianus, Ranz. C. vulturinus, Temm. Pl. col. 51. Gypagus californianus, Vieill. Catharista californianus, $G$. $R$. $G r$. Sarcoramphus californianus, Vig. Licht. F. von Calif. t. 1. Berl. Trans. 1838.

S. IV. of N. America, (California.)

5. Sarcoramphus atratus.

B. M.

Vultur urubu, Vieill. V. iota, Wils. Amer. Orn. pl. 75, f. 2.

V. atrata, Bartr. V. brasiliensis, Ray. Cathartes foetens, Ill.

C. atratus, Rich. et $\mathrm{Sw}$. C. brasiliensis, $\mathrm{Pr}$. B. C. urubu, Less. Catharista urubu, Vieill. C. iota, $\operatorname{Pr} . B$. Percnopterus uruba, Steph. Ois, de l'Amer. Sept. t. 2.

S. of N. America. 
6. Sarcoramphus iota.

B. M.

Vultur iota, Mol. V. aura, L. Wils. Amer. Orn. pl. 75, f. 1. $P l$. enl. 187.? Cathartes aura, $I l l$. C. septentrionalis, $P r$. Max. C. ruficollis, Spix. C. jota, Pr. B. Catharista aura, Vieill. Percnopterus aura, Steph. Ois. de l'Amer. Sept. t. 2, bis. C. Burrovianus, Cass.

N. and S. America.

\section{ii. Vulturine.}

Vulturinæ, Pr. B. 1838-49-50; G. R. Gr. 1840-41-44; Bl. 1849: Cass. 1849.

Gypinæ, Bl. 1849 ; Cass. 1849.

\section{Vultur.}

a. Vultur, Mahr. 1752 ; Linn. 1756.

Egypius, Sav. 1809.

Polypteryx, Hodgs. 1844.

7. VULTUR MONACHUS.

B. M.

Vultur monachus, L. Edw. B. pl. 290. V. cinereus et V.cristatus, Gm. V. vulgaris, Daud. V. arrianus, Lapeyr. V. niger, Vieill. Egypius niger, Sav. V. imperialis, Temm. Pl. col. 426. Polypteryx cupido $\mathrm{v}$. cinereus, Hodgs. Pl. enl. 425. Gould, B. of Eur.pl. 2. Disc. de l'Egypte, Ois, t. 11. Naum. Vog. t. 1.

S. Europe, Asia, N. E. Africa.

8. Vultur occipitalis.

B.M.

Vultur occipitalis Burch. V. yalericulatus, Temm. Pl. col. 13.

Levaill. Ois. de l Afr. t. 12. V. eulophus, Ehrenb. Riipp. Atlas, t. 22 .

N. E. and S. Africa.

b. Otogyps, G. R. Gr. 1841.

Hemigyps Hodgs. 1844.

Vultur, p. Pr. B. 1850.

9. Vultur auricularis.

Vultur auricularis, Daud. Leraill, Ois, de l'Afr. 9. V.M. pius, Temm. Pl. col 407 V nubicus, $H$. Smith . ægyauricularis, G. R. Gr. Griff. An. Kingd. 1, pl. p. 64 .

Greece, N. E. and S. Africa. 
10. Vultur calvus.

B. M.

Vultur calvus, Scop. Sonn. Voy. t. 104. V. Ponticerianus, Daud. Hemigyps ponticerianus, Hodgs. Otogyps calvus, $G$. R. Gr. Ann. du. Mus. 1, t. 20. Shaw, Nat. Misc. pl. 941. Pl. col. 2. Gr. Ill. Ind. Zool. pl. 15, f. 2.

India.

c. Gyps, Sav. 1809.

11. Vultur Fulvus.

B. M.

Vultur fulvus, $G m$. Pl. enl. 426. V. leucocephalus, Mey.

V. percnopterus et V. vulgaris, Daud. V. castaneus, Shaw.

V. trincalos, Bechst. V. persicus, Pall. V. albicollis, Linderm. $V$. fulvus occidentalis et $V$. fulvus Ruppelii, Schl. Rupp. Zool. Atl. t. 32 . Gyps vulgaris, Sav. G. fulvus, $G$. $R$. $G r$. G. indicus, G. occidentalis et G. Kolbii, $P r . B$. Vultur indicus, Temm. Pl. col. 26. V. Kolbii, Daud. Levaill. Ois. de IAfr.t. 10. V. Ruppelli, Natt. Gould,B. of Eur.pl. 1. Shau, Nat. Misc. pl. 141. Yarr. Brit. B.2 edit. i. fig. p. 1. Naum. Vog. t. 2.

Ireland! S. and W. Europe, India, Africa.

12. VULTUR BENGALENSIS.

B. M.

Vultur bengalensis, Gm. Lath. Gen. Syn. pl. 1. F. chaugoun, Daud. Levaill. Ois. de 'Afr.t. 11. V. leuconotus, Gr. Ill. Ind. Zool. pl. 14. V. indicus, Scop.? Sonn. Voy. t. 105.? Gyps hengalensis, G. R. Gr. Gr. Ill. Ind. Zool. pl. 15.

India and Senaar (?)

13.? VCLTUR TENUIROSTRIS.

Vultur tenuirostris et $\mathrm{V}$. tenuiceps, Hodgs. $\mathrm{V}$ indicus, B. Gyps tenuirostris, G. R. Gr. and Mitch. Gen. of B. pl. 3. India.

G. bengalensis, jun. $\operatorname{Pr} . B$. G. indicus, $B l$.

\section{iii. Neophronin.e.}

Neophroninæ, G.R. Gr. 1848.

Neophrinæ, Cass. 1849.

Cathartinæ, p. G. R. Gr. 1840.

Racaminæ, G. R. Gr. 1840-41.

Gypohieracinæ, G. R. Gr. 1844. Pr.B. 1849-50. 


\section{NEOPHRON.}

a. Neophron, Sav. 1809.

Percnopterus, Cuv. 1817.

14. NeOphron PERCNopterus.

B. M.

Vultur percnopterus, L. Pl. enl. 427, 429. V. stercorarius, Lapeyr. V. leucocephala, Briss. V. fuscus, Bodd. Gm. V. albus et V. gingianus, Daud. V. meleagris, Pall. Cathartes percnopterus, Temm. Percnopterus ægyptiacus, Steph. Neophron percnopterus, Sav. Levaill. Ois. de l'Afr. t. 14. Lath. H. of B. pl. 5. Gould, B. of Eur. pl. 3. Jard. \& Selby's Ill. of Orn. pl. 33. Naum. Vög. t. 3. Yar. Brit. B. 2 edit. i. fig. pp. $6,10$.

England! S. Europe, Africa, India.

b. Caprornis, Kaup, 1849.

15. NeophroN pileatus.

B. M.

Vultur pileatus, Burch. Cathartes monachus, Temm. Pl. col. 222. Neophron carunculatus, A. Smith. N. pileatus, G. R. Gr. Percnopterus niger, Less. P. monachus, Steph.

S., W. and N.-E. Africa.

\section{GypohiERAX.}

Gypohierax, Rüpp. 1835.

Racama, Gr. 1840.

16. Gypohiebax ANGOLExsis.

B. M.

Falco angolensis, Gm. Shaw, Lev. Mus. pl. p. 153. Penn. Tour in Wales, i. pl. 19. Vultur angolensis, Lath. Gypaëtus angolensis, Daud. Polyborus? hypoleucus, Benn. Jard \& Selby's Ill. of Orn. n. s. pl. 13. Racama angolensis, Gr. Gypohierax angolensis, Ruipp. Cathartes (Gypohierax) angolensis, Kaup.

W. Africa. 


\section{FALCONIDÆ.}

Falcunidæ, Leach. Vig. 1825. Pr. B. 1831-38-49-50. Sw. 1831. -37. G. R. Gr. 1840-41-44. Bl. 1849. Fulconides, Sundev. 1835.

\section{i. Polyborina.}

Polyborinæ, G. R. Gr. 1840-41-44. Pr. B. 1849-50. Bl. 1849. Ayuilina, Vig. 1825.

Falconinæ, p. Pr. B. 1831.

Cymindinæ, p. Sw. 1837.

Buteoninæ, p. Kaup, 1847.

\section{IBYCTER.}

a. Ibycter, Vieill. 1816.

Gymnops, Spix, 1824.

17. IBYCTER A MERICANUS.

Falco americanus, Bodd. Plenl. 417. F B. M. F. nudicollis, Daud. F. formosus, Lath. Caracara aquilina, Cuv. Gymnops aquilinus, Spix. Ibycter leucogaster, Vieill. I. americanus, G.R. Gr. I. aquilinus, Steph. Polyborus (Daptrius) nudicollis, Kaup. Gal. des Ois. t. 6. Shaw, Nat. Misc. pl. 485.

S. America, (Cayenne, Brazil.)

18. IBYCTER GYMNOCEPHALUS.

Ibycter gymnocephalus, D'Orb. \& Lafr.

S. America, (Bolivia).

b. Daptrius, Vieill. 1816.

19. IBYCter ater.

Daptrius ater, Vieill Gal. B. M. pl. 32. Falco aterimus, Temm. Pl. Shaw, Gen.Zool.xiii. aterrimus, Cuv. Gymnops aterrimus, col. 37, 342. Caracara trius) aterrimus, Kaup. Ibycter ater, $S w$. Ibycter (DapS. America, (Guiana, Brazil.) 
20? Ibycter faschatus. Gymnops fasciatus, Spix, Av. Bras. t. 4. Caracara fasciatus,
Cuv.

S. America, (Brazil.)

\section{Milvago.}

a. Milvago, Spix, 1824.

Parasifalco, Less. 1837.

21. Milvago chimachima.

B. M.

Polyborus chimachima, Vieill. P. crotophagus, Pr. Max. P. degener, Steph. Milvago ochrocephalus, Spix, Av.Bras. t. 5. M. chimachima, G. R. Gr. Falco degener, Ill. Parasifalco, chimachima, Less. Gymnops strigilatus, Spix, Av. Bras. t. 4. Haliaëtus ochrocephalus, Cuv. H. chimachima, Less. Polyborus (Milvago) degener et Ibycter (Milvago) chimachima, Kaup. Jard. \&. Selby's Ill. of Om. pl. 2.

America, (Brazil).

22. Milfigo chimango.

Aquila pezoporus, Meyen N. M. (IIilva (Milvago) pezoporus et Ibycter (Milvago) chimango, Kaup. Haliaëtus chimango et Parasifalco chimango, Less. Milvago chimango, G. R. Gr.

S. America, (Brazil, Chili).

b. Senex, Gr. 1839 .

Aëtotriorchis, Kaup, 1844.

Polyborus, p. Pr. B. 1850 .

23. Milvago australis.

Falco australis et $\mathrm{F}$ ambustus, nove novæ zealandiæ, Temm. Vultur plancus o , Forst. Circaëtus noræ zealandiæ, Cuv. C. antarcticus, Less. Ibycter (Aëtotriorchis) australis, Kaup. Milvago leucurus, et M. australis, G. R. Gr. Polyborus australis, Pr. B. P. noræ zealandiæ, Steph. Jard. \& Selby, Ill. of Orn. n. s. pl. 5.

S. America and Falkland Islands. 
c. Phalcobænus, D'Orb. \& Lafr. 1837.

24. Milvago megalopterus.

B. M. Aquila megaloptera, Meyen, Nov. Acta, (1834) t. 8. Phalcobænus monlanus, D'Orb. \& Lafr. Voy. dans l'Amer. Mer. Ois. t. 2, f. 1, 2. P. megalopterus, $\boldsymbol{P r} . \mathbf{B}$. Ibycter (Phalcobænus) montanus, Kaup. Milpago megalopterus, G. R. Gr. M. albigularis, Gould, Voy. of Beagle, Birds, pl. 1. M. montana, Bl.

S. America, (Chili).

\section{Polyborus.}

Polyborus, Vieill. 1816.

Caracara, Cuv. 1817.

25. Polyborus Brasiliensis.

B. $M$.

Fulco brasiliensis, Gm. F. plancus, Mill. Illustr. pl. 17. F. cheriway, Jacq. F. tharus, Mol. Vultur plancus, Forst. Polyborus vulgaris et $\mathrm{P}$. cheriway, Vieill. Caracara brasiliensis, Cuv. C. vulgaris, Less. Polyborus brasiliensis, Sw. Zool. 1ll. n. s. pl. 2. Gal. des Ois. t. 7. Spix, Av. Bras, t. 1a.

S. America, S. of N. America, and Hermite Island:

\section{ii. Buteonin瓜.}

Buteoninæ, Sw. 1837. Pr. B. 1838-49-50. G. R. Gr. 184041-49. Bl. 1849 .

Falconinæ, p. $\operatorname{Pr} . B .1831$.

Thrasaëtinæ, p. Bl. 1849.

1. Buteo.

a. Buteo, Cuv. 1799-1800.

26. Bute o Vulgaris.

Falco buteo, $L . \quad P l$. enl 419 . sicolor, Gm. F. vulgaris, Bechst B Auteo, Pall. Buteo tus, Vieill B . B. CesciaB murl. B. albus, Daud. B. septentrionalis, B. mediun et . Brehm. Bulgaris japonicus, Temm. \& Schl. B. fuscus, Macgill. Fanna Jap. t. 6, B. Gould, B. of Eur. pl. 14. Naum. Vog. t. 32, 33. Yarr. Brit. B. 2 edit. i. fig. p. 82. Great Britain, Europe, Asia minor, Japan, N. Africa.

27 ? BUTEO PYGMæUS.

Buteo pygmæus, $B l$.

Tanesserim. 
28. Buteo rufinus.

B. $\mathbf{M}$.

Circus rufinus, Rüpp. Zool. Atlas, t. 27. Buteo canescens, Hodgs. B. longipes et B. rufiventer, Jerd. B. rufinus, Kaup. B. vulgaris, $B l$. B. circentis, Levaill.

India and N.-E. Africa.

29. BUTEO JACKAL.

B. M.

Falco jackal, Daud. Levaill. Ois. de ' Afr. t. 16. Buteo jackal, Cuv.

S. Africa.

30. Bdteo hugur.

B. M.

Falco augur et F. hydrophilus, Rüpp. Abyss. t. 16, 17. Buteo augur, Ruipp.

N.-E. Africa, (Abyssinia).

31. Buteo plumipes.

B. M.

Circus plumipes et Buteo plumipes, Hodgs.

India, (Nepaul).

32. Buteo? Desertorum.

Falco desertorum, Daud. Levaill. Ois. de l'Afr. t. 17. Buteo desertorum, Vieill. B. capensis, p. Temm. et Schl. Tinnunculus desertorum, $G . R$. $G r$.

S. Africa.

\section{b. Pœcilopternis, Kaup, 1847. Craxirex, Gould, 183٪? Buteo, p. Pr. B. 1849.}

33. Buteo borealis.

B. M.

Falco borealis, F. leverianus, F. variegatus, F. cinereus, F. jamaicensis et F. obsoletus, Gm. F. ferrugineocauda, Vieill. F. Harlani, Audub. B. of Amer. pl. 86. Buteo borealis, B. ferrugineocaudus, B. cinereus, B. obsoletus, B. americanus, B. fulvus, Vieill. B. vulgaris, Rich. \& Sw. Fauna Bor.-Amer. pl. 27. B. Swainsoni, Pr, B. B. montana, Nutt. B. ventralis, Gould. B. ferrugineocaudus, Cuv. Astur borealis et A. leverianus, Cuv. Accipiter ruficaudus, Vieill. Ois. de l'Amer. Sept. t. 14. Buteo (Pcecilopternis) borealis, Kaup. Circus variegatus, Vieill. Edw. B. pl. 53. Wils. Amer. Orn. pl. 52, f. 1, 2. Vieill. Ois. de l'Amer. Sept. t. 6. Audub. B. of Amer. pl. 372. Gosse, B. of Jam. pl. 2.

N. and S. America and W. Indies. 
34. Buteo lineatus.

B. M.

Falco lineatus, Gm. Wils. Amer. Orn. pl. 53, f. 3. F. hyemalis, Wils. Amer. Orn. pl. 35, f. 1. F. buteoìdes, Nutt. Buteo lineatus et B. fuscus, Vieill. B. hyemalis, Less. Nisus lineatus et N. hyemalis, Cuv. Circus hyemalis, $\operatorname{Pr}$. B. Astur? hyemalis, Jard. Buteo (Pocilopternis) lineatus, Kaup. Vieill. Ois. de l'Amer. Sept, t. 5. Audub. B. of Amer. pl. 56.

N. America \& California.

35. Beteo PENNSYLtanicus.

B. MI.

Falco pennsylvanicus, Wils. Amer. Orn, pl. 54, f. 1. F. latissimus, Ord. F. Wilsoni, $\operatorname{Pr}$. B. Astur pennsylvanicus, $C u v$. A.? latissimus, Jard. Buteo pennsylvanicus, $P r . B$. Sparvius platypterus, Vieill. Buteo (Pæcilopternis) Wilsonii, Kaup. Audub. B. of Amer. pl. 91.

N. and S. America.

36. Buteo galapagoensis.

Craxirex galapagoensis, Gould, Voy. of Beagle, pl. 2.

Buteo galapagoensis, G. R. Gr.

Islands of Galapagos.

c. Tachytriorchis, Kaup, 1844.

37. Buteo erythronotus.

B. M.

Haliaëtus erythronotus, King. Falco polysoma, Quoy \& Gaim, Voy. de l'Uraine, Ois. t. 14. Aquila braccata, Meyen, Nova Acta, (1833) t. 8. Buteo tricolor et B. unicolor, D'Orb. \& Lafr. Voy. dans l'Amer. Ois.t. 3, f. I, 2. B. varius, Gould. B. braccata, Cab. B. erythronotus, G. R. Gr. B. polysoma, Less. Astur polysomus, Cuv. Buteo ('Tachytriorchis) pterocles, p. et Buteo (Pœcilopternis) erythronotus, Kaup. Tachytriorchis erythronotus, $\operatorname{Pr}$. $B$.

S. America, (Chili, Str. of Magellan, Caraccas), Falkland Islands.

38. Buteo PTERocles.

B. M.

Falco pterocles, Temm. Pl. col. 56, 139. Buteo pterocles, Cuv. B. albicaudatus et B. nigricollis, Vieill. B. albicanda, Less. Tr. d'Orn.t. 15, f. 2. Spizaëtus leucurus, Vieill. Buteo ('Tachytriorchis) pterocles, Kaup. Tachytriorchis pterocles, Pr. B.

S. America, (Brazil). 
39. Bdteo albonotatus. B. M.

Buteo albonotatus, $G . R$. Gr. B. (Tachytriorchis) albonotatus, Kaup. Tachytriorchis albinotatus, $\operatorname{Pr} . B$.

Mexico.

40. Buteo leucops.

B. M.

Buteo leucops, G.R. Gr. B. (Pœcilopternis) infulatus, Kaup. Tachytriorchis leucops, $\operatorname{Pr} . B$.

Islands of Galapagos.

d. Gypoictinia, Kaup, 1845.

41. Buteo melanosternum.

Buteo melanosternum, Gould, B. of Austr. pl. 20. Milrus (Gypoictinia) melanosternum, Kaup. Gypoictinia melanosternum, $\operatorname{Pr} . B$.

New South Wales, S. and W. Australia.

e. Buteogallus, Less. 1831.

Ichthyoborus, Kaup, 1845.

42. Buteo equin octialis.

B. M.

Falco æquinoctialis, Gm. F. buson, Daud. Levaill. Ois, de l'Afr. t. 2l. Polyborus buson, Vieill. Aquila urubitinga, Spix, Av. t. 1 ? Buteogallus cathartoïdes et B. buson, Less, Pr. B. Circaëtus solitarius, Tschudi ? Pr. B. Buteo (Buteogallus) buson et Buteo (Ichthyoborus) æquinoctialis, p. Kaup.

S. America, (Brazil, Guiana).

43. BUTEO NigRiColLis.

B. M. Falco nigricollis, Lath. F. busarellus, Daud. Levaill. Ois. de l'Afr. t. 20. F. melanobronchos, Shaw. Aquila milvoïdes, Spix, Av. t. 1, $d$. Buteo busarellus, Cuv. B. nigricollis, $G$. R. Gr. Morphnus milvoïdes, Cuv. Circus busarellus et B. leucocephalus, Vieill. Buteo (Icthyoborus) busarellus et Buteo (Ichthyoborus) æquinoctialis, p. Kaup. Buteogallus busarellus, Pr. $B$.

S. America, (Brazil). 


\section{f. Leucopternis, Kaup, 1847.}

Pseudastur, Bl. 1848.

44. Buteo melayops.

B. M.

Falco melanops, Lath. $P l$. col. 105. Buteo melanops, G. R. $G r$. Sparvius melanops, Vieill. Asturina (Leucopternis) melanops, Kaup. Leucopternis melanops, $\operatorname{Pr}$. $B$.

S. America, (Guiana).

45. Buteo Kuhlit.

B. M.

Leucopternis Kuhli, Pr. B. Consp. p. 19.

S. America.

46. BU'TEO AlBicollis.

B. M.

Falco albicollis, Lath. F. picatus, Shaw. Buteo albicollis, G. $\boldsymbol{R}$. $G r$. Asturina (Leucopternis) albicollis, $\boldsymbol{K}$ aup. Leucopternis albicollis, $\boldsymbol{P r}$. $B$.

S. America, (Cayenne).

47. Buteo scotopterus.

B. $\mathbf{M}$.

Falco scotopterus, $\operatorname{Pr}$. Max. F. lacernulatus, Temm. Pl. col. 437. Buteo lacernulatus, Cuv. Asturina (Spizageranus) scotoptera et A. (Leucopternis) scotopterus, Kaup. Leucopternis lacernulatus, $\operatorname{Pr}$. $B$.

S. America, (Brazil).

48. Buteo polionotus.

B. $\mathrm{M}$.

Buteo polionotus, G. R. Gr. Buteo melanotus, Vieill.? Leucopternis polionotus, $\operatorname{Pr} . B$.

S. America, (Guiana).

49. Buteo pecilonotus.

Buteo pocilonotus, Cuv. B. brachyurus, Vieill.? B. Ghiesbreghtii, Dubus, Esquis. Orn. t. 1. Falco pœcilonotus, Temm. $P l$. col.9. Pseudastur pœcilonotus, $B \boldsymbol{B}$. Asturina pœcilonotus et A. (Leucopternis) pœecilonotus, Kaup. Leucopternis pœcilonotus, $P r . B$.

S. America, (Guiana).

50.? Buteo abBreviatus.

Buteo abbreviatus, Licht. R. Schomb. Reis. iii. p. 739.

S. America, (British Guiana). 
g. Butaquila, Hodgs. 1844.

Hemiaëtus, Hodgs. 1844.

51. Buteo strophiatus.

B. $M$.

Heminëtıs strophiatus, Hodgs. Buteo leucocephalus et B. aquilinus, Hodgs. B. hemilasius, Temm. \& Schl. Fauna Jap. t. 7.? Butaquila leucocephala, Hodgs. Archibuteo strophiatus, G.R. Gr. "Falco asiaticus, Lath." Bl. Buteo (Arclibuteo) strophiatus, Kaup.

Nepal and Japan.

52. Buteo HeMtPtilopus.

Archibuteo hemiptilopus, Bl. A. cryptogenys, Hodgs. A. griseogenys, Bl.? Calc. Journ. of Nat. Hist. 1847, pl. 3, f. 1 . Tibet, Sikim?

\section{h. Archibuteo, Brehm, 1828. \\ Triorchis, Kaup, 1829. \\ Butaëtes, Less, 1831.}

53. Buteo lagopus.

B. M.

Falco lagopus, Brün. F. plumipes, Daud. F. sclavonicus, Lath. F. pennatus, Cuv. Accipiter lagopus, Pall. Archibuteo lagopus, G. R. Gr. A. planiceps et A. alticeps, Brehm. Butaëtes buteo et B. lagopus, Less. B. Lessonii, A. Smith. B. lagopus, $\operatorname{Pr}$. B. Buteo lagopus, Leach. Triorchis lagopus, Kaup. Naum. Vög. t. 34. Gould, B. of Eur. 1, pI. 15. Yarr. Brit. 2 edit. i. fig. p. 87.

Great Britain, Europe and S. Africa.

54. Buteo Sancti Johansis.

B. $M$.

Falco Saneti Johannis et F. novæ terræ, $G m$. F. niger et F. lagopus, Wils. Amer. Orn. pl. 53, f. 1,2, and pl. 33, f. 1. F. spadaceus, Forst. Buteo ater et B. Sancti Johannis, Vieill. B. niger, Cuv. Butaëtes Sancti Johannis, Less. Archibuteo Sancti Johannis, G. R. Gr. Rich. \&. Sw. Fauna Bor.-Amer. pl. 28. Audub. B. of Amer. pl, 166, 422.

N. America.

55. Buteo ferrugineus.

Falco (Buteo) ferrugineus, Licht Archibuteo

Gr. \& Mitch. Gen. of Birds,

Buteo Aro Mexico. 
Examine, Buteo solitarius, Peale, U. S. Expl. Exped. viii. p. 62, pl. 16.

Island of Hawii.

iii. Aquilinfe.

Aquilinæ, Sw. 1837 ; Pr. B. 1838-49-50; G.R. Gr. 1841-45;

Kaup, 1847 ; Bl. 1849.

Aquilina, Vig. 1824.

Falconinæ, Pr. B. 1831.

Buteoninæ, p. et Accipitrinæ, p. Sw. 1837.

Aquilæ et Astures, p, Kaup, 1843.

Accipitres, p. Kaup, 1847.

Circaëtinæ, Thrasaëtinæ et Haliaëtinæ, Bl. 1849.

\section{Aquila.}

a. Aquila, Møhr, 1752 .

Aëtos, Nitzsch, 1840.

56. AqUILA chrYsaEtos.

B. M.

Falco chrysaëtos, F. melanaëtos, F. fulvus, et F. canadensis, L. Pl. enl. 409, 410. F. niger et F. americanus, Gm. F. melanonotus, Lath. F. regalis, Temm. Aquila chrysaëtos et A. nobilis, Pall. A. fulva, Meyer. A. regia, Less. A. melanaëtus, Brehm. A. daphænia, Hodgs. Wils. Amer. Orn. pl. 55, f. 1. Gould, B. of Eur. pl. 6. Naum. Vög. t. 8, 9. Yarr. Brit. B. 2 edit. i. fig. p. 11.

Great Britain, Europe, N. Asia and N. America.

57. Aquila mogidnik.

B. M.

Falco mogilnik, S. G. Gm. A. imperalis, Cuv. A. heliaca, Sav. A. chrysaëtos, Leisler. A. bifasciata, $G r$. A. nipalensis et A. crassipes v. nævia, Hodgs. Falco imperialis, Bechst. Pl. col. 151, 152. Disc. de l'Egypte, t. 12. Gould, B. of Eur.pl. 5. Gr. Ill. Ind. Zool. i. pl. 17, pl. 28. As. Res, ii. pl. Naum. Vög.t. $6,7$.

E. Europe, Central Asia and N. Africa.

58. Aquila N NEVIA.

B. M.

Falco nævius et F. maculatus, $G m$. Aquila næria, Meyer. A. pomarina, Brehm. A. planga, Vieill. A. bifasciata, Hornsch. A. melanaëtos, Sav. A. clanga, Pall. A. vittata, Hodgs. Spizaëtus fuscus, Vieill. Disc. de $T$ Egypte, t. I, t. 2, f. 1. Gould, B. of Eur. pl. 8. Naum. Vög. t. 10, 11. Yarr. Brit. B. 2 edit. i. fig. p. 10**

Ireland! E. Europe, Central and N. Asia and N. Africa. 
59. Aquila hastata.

Morphnus hastatus, Less. Spizaëtus punctatus, Jerd. Limnaëtus unicolor et Aquila hastata, $B l$.

India.

60. Aquila netiojdes.

B. $M$.

Falco nævioïdes, Cuv. F. rapax, Temm. $P l$. col. 455. F. albicans, Rüpp. F. senegallus, Cuv. F. belisarius, Levaill. Expl. Sci. de l'Algir. t. 2. F. obsoletus, Licht.? Aquila nævioïdes et A. senegallus, Cuv. A. choka, A. Smith. A. fulvescens, A. fusca et A. punctata, Gr. A. vindhiana, Frankl. A. rapax, Less. Gr. Ill. Ind. Zool. pl. 29, 27, 16.

S. and N. Africa, India.

b. Pseudaëtus, Hodgs. 1844.

Tolmaëtus, $B l$. 1845.

Eutolmaëtus, $B l .1848$.

61. Aquila Bonellit.

B. M.

Falco Bonellii, Temm. Pl.col. 288. F. ducalis, Licht. Aquila Bonellii, Pr. B. A. intermedia, Bonelli. A. bifasciata, Vieill. A. vipalensis v. rubriventer, Hodgs. A. minima, Cetti. Tolmaëtus Bonellii et Eutolmaëtus Bonellii, $B l$. Nisaëtus grandis, Hodgs. N. niveus, Jerd. Aquila (Hieraëtus) Bonellii, Kaup. Gould, B. of Eur. pl. 7.

Middle of Europe, N. Africa and Nepal.

c. Hieraaëtus, Kaup, 1844.

Hieraëtus, Kaup, 1847.

62. Aquila pennata.

B. M.

Falco pennatus, Gm. Pl. col. 33. Gould, B. of Eur. pl. 9. Aquila pennata, Cuv. A. minuta, Brehm. Spizaëtus milvoïdes, Jerd. Aquila (Hieraëtus) pennata, Kaup. Hieraëtus pennatus, $B l$.

Europe, Africa and India.

63. Aquila morphnoides.

Aquila morphnoïdes, Gould, B. of Austr.pl. 2. Aquila (Hieraëtus) morphnoides, Kaup.

New South Wales and S. Australia. 
d. Pteroaëtus, Kaup, 1844.

64. Aquila vulturina.

B. M.

Falco vulturinus, Daud. Levaill. Ois. de l'Afr. pl.6. F. niger, James. Aquila vulturina et A. Verreauxii, Less. Cent. Zool. t. 38. Gypaêtos cafer et G. vulturina, Temm. Haliaëtus vulturinus, Cuv. Aquila (Pteroaëtus) vulturina, Kaup.

S. and N.-E. Africa.

e. Heteropus, Hodgs. 1842.

Neopus, Hodgs. 1847.

Onichaaëtus, Kaup, 1844.

Onychaëtus, Kaup, 1847.

Ictinaëtus, Jerd. 1844.

65. Aquila malayensis.

B. M.

Falco malayensis, Reinw. Pl. col. 117. Aquila malayensis,

Cuv. A. malayana, Less. A. (Heteropus) pernigra et Neopus pernigra, Hodgs. Nisaëtus? ovivorus et Ictinaëtus malayensis, Jerd. Aquila (Onychaëtus) malayensis, Kaup.

Malacea and Nepal.

f. Uroaëtus, Kaup, 1844.

Uraëtus, Kaup, 1845.

66. Aquila audax.

B. $M$.

Vultur audax, Lath. Gould, B. of Austr. 1, pl. 1. Falco fucosus, Cuv. Pl. col. 32. Aquila audax, Lath. A. fucosa, Cuv. A, albirostris, Vieill. A. cuneicaudata, Brehm. Aquila (Uroaëtus) audax, Kaup.

Australia (except the North).

\section{Spizaetus.}

a. Spizaëtus, Vieill. 1816.

Plumipeda, Flem. 1822.

Harpyia, Spix, 1824.

67. Spizaetus ornatus.

B. $\mathrm{M}$.

Falco ornatus et F. manduyti, Daud. F. superbus, F. coronatus et F. fastuosus, Shaw. Spizaëtus ornatus, Vieill. Gal. des Ois. t. 21. Morphnus ornatus, Cuv. Plumipeda superbus, Flem. Harpyia braccata, Spix. H. ornata, Sw. Aquila urutaurana, Dum. Levaill. Ois. de l'Afr. t. 26. Spix. Av. Bras. t. 3.

S. America (Brazil). 
68. Spizaetus bellicosus.

B. M.

Falco bellicosus, Daud. Levaill. Ois, de l'Afr. t. 1. F. armiger, Shaw. Aquila bellicosa, Dum. A. armigera, Cuv. Spizaëtus bellicosus, Kaup. A. Smith, Ill. S. Afr. Zool. pl. 42.

s. Africa.

69. Spizaetus coronatus.

B. M.

Falco coronatus, $L$. Edw. Birds, pl. 224. F. albescens, Daud, Levaill. Ois. de ' Afr. t. 3. Aquila coronata, A. Smith. Morphnus albescens, $C u v$. Spizaëtus coronatus et S. albescens, Vieill. A. Smith, Ill. S. Afr. Zool. pl. 41.

S. Africa.

\section{b. Pternura, Kaup, 1845. \\ Pterura, G.R. Gr. 1848.}

70. Spizaetus tyrannus.

Falco tyrannus, $\mathrm{Pr} . M a x . P l . c o l .73$. Spizaëtus tyran G. R. Gr. Morphnus tyrannus, Cuv. Spizaëtus (Pternura) tyrannus, Kaup. S. braccatus, $O$ Des Murs, lconogr. Ornith. t.

S. America (Brazil).

71? Spizaetus Isidori. Aquila Isidori, O Des Murs, Iconogr. Ornith. t. 1. Spizaëtus,
Isidori, G. R. Gr.

S. America.

c. Spizastur, Less. 1837.

72. Spizaetus melanoleucus.

Buteo melanoleucus, Vieill. Gal, des Ois. t. 14 lus, Cuv. Falco atricapillus, $C$. T. 14. B. atricapilzastur atricapillus, Less. et Spizaëtus (Spizastur) Morphnus (Spizastur) atricapillus, nolencus, $G . R$. $G r$.

S. America (Cayenne). 


\section{d. Lophaëtus, Kaup, 1847.}

73. Spizaetus occipitalis.

Falco occipitalis, Daud. Levaill. Ois. de l'Afr. t. 2. F. senegalensis, Daud. Spizaëtus occipitalis, Vieill. Morphnus oceipitalis, Cuv. Harpyia occipitalis, $S w$. Spizaëtus (Lophä̈tus) occipitalis, Kaup.

S. and N.-E. Africa.

e. Limnaëtus, Vig. 1830.

Nisaëtus, Hodgs. 1836.

74. Spizaetus cirrhatus.

B. M.

Falco cirhatus et F. ceylonensis, Gm. F. caligatus, Raffl. F. lymnaëtus, Horsf. Pl. col. 134. F. niveus, Shaw, Temm. Pl. col. 127. F. cristatellus, Temm. Pl. col. 282. F. Latbami, Tick. Morphnus cristatellus, M. niveus et M. lymnaëtus, Cuv. Spizaëtus cirrhatus, Kaup. S. cristatellus, Jard. \& Selby. S. lymnaëtus, $B l$. S. orientalis et S. lanceolatus, Temm. \& Schl. Fauna Jap. t. 3, 7? Nisaëtus pallidus, N. pulcher et N. nipalensis, Hodgs. N. alboniger, $\boldsymbol{B} l$. Limnaëtus Horsfieldii, L. unicolor, et L. caligatus, Vigors. Morphnus cristatellus, M. niveus, et M. lymnaêtus, Less. M. (Lymnaëtus) caligatus, Kaup. Jard. \& Selby, Ill. of Orn. pl. 1. As. Res. 1833, pl. 1 .

India and its Archipelago.

75. Spizaetus Kieneri.

Astur Kieneri, Gerv. Mag. de Zool. 1835. Ois. t. 35. Spizaëtus albogularis, Tick. S. Kieneri, G.R. Gr. India.

\section{Morphnus.}

a. Morphnus, Cuv. 1817.

Morphinus, Flem. 1822.

76. Morphnus guianensis.

Falco guianensis, Daud. Less, Tr, d Orn t B. M. nini, Shaw MLrphnus cristatus, Jess M. SonSpizaëtus variegatus, Vieill. Harpyia guianensis, $S w$. Asturina (Thrasaëtus) guianensis, et A. (Morphnus) guianensis, Kaup.

S. America (Guiana). 
b. Urubitinga, Less. 1837.

Spizageranus, Kaup, 1844.

Hypomorphnus, Cab. 1844.

77. Morphnus trubitinga.

B. M.

Falco urubitinga, Gm. F. longipes, $I l l$. Morphnus urubitinga, Cuv. Aquila picta, Spix. Av. Bras. t. 1? Pandion fulvus, Vieill. Spizaëtus niger, S. ater, et S. maculatus Vieill. Harpyia urubitinga, Sw. Hypomorphnus urubitinga, $C a b$. Asturina (Spizageranus) urubitinga et A. (Rupornis) urubitinga, Kaup. Urubitinga longipes, $\operatorname{Pr}$. $B$.

S. America (Brazil, Cayenne), and W. Indies?

78? Morphyes LeUCUrus.

Hypomorphnus leucurus, Lafr. Rev. et Mag. Zool. 1850, p. 388 .

S. America.

79. Morphnus meridionalis.

B. M. Falco meridionalis, Lath. F. rutilans, Licht. Pl. col. 25. Aquila buson, Spix. Circus rufulus, Vieill. C. rutilans, Steph. Hypomorphnus rutilans, Cab. Buteo rutilans, Less. Asturina (Rupornis) meridionalis, Kaup. Morphnus meridionalis, G. R. Gr. Urubitinga meridionalis, $\operatorname{Pr} . \boldsymbol{B}$.

Mexico and S. America (Brazil, Paraguay, Guiana).

80. Morfunus mexicanus.

Morphnus mexicanus, Dubus.

Mexico.

\section{Thrasaetus.}

a. Thrasaëtus, G. R. Gr. 1837.

Harpia, Vieill. 1816.

Harpyia, Cuv. 1817.

81. Thrasaetus harpya.

B. M.

Vultur harpyia, $L$. Falco harpyia, F. imperialis, F. calquin, F. regalis et F caracea? Shaw, Gen. Zool. 1, pl. 15. F. destructor, Daud. Pl. col. 14. F. Jacquini et F. cristatus? Gm. Gypaëtus harpyia et G. coronatus, Daud. Morphnus harpyia, Cab. Harpyia destructor, Cuv. H. ferox, Less. H. maxima, Vieill. H. imperialis, Steph. Thrasaëtus hurpyia, G. R. Gr. Asturina (Thrasaëtus) harpyia, Kaup. Less. Tr. Orn. t. f. 1. Less. Compl. Buff: Ois, t. 1.

S. America (Guiana). 
b. Harpyhaliaëtus, Lafr. 1842.

82. Thrasaetos? coronatus.

B. M. Harpyia coronata, Vieill. Falco coronatus, Temm. $\mathrm{Pl}$. col.234. Circaëtus coronatus, Cuv. Asturina (Thrasaëtus) Azaræ, Kaup. Harpyhaliaëtus coronatus, Lafr. Thrasaëtus coronatus, Pr. B. Cymindis coronatus, Steph. Haliaëtus unifasciatus, G. R. Gr.

S. America (Paraguay, Brazil, La Plata).

\section{Circaetus.}

a. Circaëtus, Vieill. 1816.

Pygargus, Koch, 1816.

83. Circaetus gallicus.

B. M.

Falco gallicus, Gm. Pl. enl. 413. F. leucopsis, Bechst. F. longipes, Nils. F. brachydactylus, Temm. Aquila brachydactyla, Meyer. A. leucamphomma, Borkh. Accipiter hypoleucus, Pall.? Circaëtus gallicus, Boie. C. leucopsis et C. anguium, Brehm. C. hypoleucos, Keys. \& Bl.? Gould, B. of Eur. 1, pl. $13 . \quad$ Naum. Vög. t. 15.

E. Europe, Arabia and N.-E. Africa.

84. Circaetus thoracicus.

Circaëtus thoracicus, Cuv. C. pectoralis, A. Smith. Aquila undulata, Licht.?

S. and N.-E. Africa.

85. Circaetus fasciolatus.

Circaëtus fasciolatus, G. R. Gr.

B. M.

S. Africa, (Natal).

86. Circaetus cinereus.

Circaëtus cinereus, Vieill. Gal. des Ois. $t .12$. C. funereus, Rüpp. Fauna Abyss, t. 14. Falco circaëtus, Temm.

N.-E. Africa. 
b. Spilornis, G. R. Gr. 1840.

Hæmatornis, Vig. 1831.

Ophaëtus, Jerd. 1844.

87. Circaetus bacha.

B. $M$.

Falco bacha, Daud. Levaill. Ois. de l'Afr. t. 15. F. bido, Horsf. Spilornis bacha et Circaëtus bacha, G. R. Gr. Hæmatornis bido, Bl. Buteo bacha, Cuv. Circaëtus (Spilornis) bacha, Kaup. Cymindis? bacha, Steph.

Java, Borneo.

88. Circaetus cheela.

B. $\mathrm{M}$.

Falco cheela, Daud. F. albidus, Cuv. Pl. col. 19. Hæmatornis undulatus, Vigors. Gould, Cent. pl. 1. H. cheela, Bl. Buteo bacha, Frankl. B. melanotis, Jerd. B. albidus, Cuv. Circaëtus cheela, G. $R$. Gr. C. nipalensis, C. mithilensis, C. tarayensis et C. maculatior, Hodgs. C.? undulatus, Jerd. Cymindis? albidus, Steph. Spilornis undulata, G. R. Gr. Circaëtus (Spilornis) cheela et C. (S) undulatus, Kaup. As. Res. 1833, pl. 2. Haliaëtus cheela, Vieill.

India.

89. Circaetus holospilus.

B. M.

Buteo holospilus, Vigors. Spilornis holospilus et Circaëtus holospilus, G.R. Gr.\& Mitch. Gen. of B. pl. 7. Circaëtus (Spilornis) holospilus, Kaup.

Philippine Islands.

c. Poliornis, Kaup, 1844 .

Butastur, Hodgs. 1844.

Bupernis, Hodgs. 184?

Buteopernis, James.?

Valeria, Boie, MSS.

90. Circaetus teesa.

B. M.

Circus teesa, Frankl. Buteo teesa, Gr. Ill. Ind. Zool. pl. 30. Astur Hyder, Sykes. Butastur teesa, Hodys. Poliornis teesa, G. R. Gr. P. fasciatus, Hay? Circaëtus (Poliornis) teesa, Kaup.

India. 
91. Circaetus liventer.

B. M.

Falco liventer, Temm. $P l$. col. 438. Buteo pallidus, Less. B. liventer, Cuv. Astur liventer et Poliornis liventer G. $R$.

Gr. Circaëtus (Poliornis) liventer, Kaup.

Malayan Archipelago.

92. Circaetus polyogenys.

B. M.

Falco poliogenys, Temm. Pl. col. 325. Buteo poliogenys, Cuv. B. poliogenys aut pyrrhogenys, Temm. \& Schl. Fauna Jap. t. 7, $B$. Poliornis indicus et P. pyrrhogenys, G. R. Gr. $\mathbf{P}$. poliogenys, $\operatorname{Pr} . \boldsymbol{B}$. Circaëtus (Poliornis) poliogenys et $\mathbf{C}$. (Poliornis) indicus, Kaup.

Philippine Islands and Japan.

93 ? Circaetus barbatus.

B. M.

Astur barbatus, Eyton. Poliomis pyrrhogenys, p. G. R. Gr. Malacca.

\section{Pandion.}

a. Pandion, Sav. 1809. Triorchis, Leach, 1816.

Balbusardus, Flem. 1828.

94. Pandion haliaetus.

B. M.

Falco haliaëtus, L. Pl. enl. 414. Gould, B. of Eur. pl. 12. F. arundinaceus, $G m$. Accipiter haliaëtus, $P$ all. Aquila balbuzard, Dum. A. haliaëtus, Meyer. Pandion haliaëtus, Cuv. P. fluviatilis, Sav. P. ichthyaëtus, Kaup. P. alticeps et P. planiceps, Brehm. P. fluvialis, Less. Balbusardus haliaëtus, Flem. Triorchis fluvialis, Leach. Falco carolinensis et $\mathrm{F}$. cayanensis, Gm. Wils. Amer. Orn. pl. 37, f. 1. Aquila piscatrix, Vieill. Ois. de l'Amer. Sept. t. 4. Pandion americanus, Vieill. Gal. des Ois. t. 11. Aquila (Pandion) haliaëta, Rich. \&. Sw. Pandion indicus, Hodgs. Naum. Vög. t. 16. Yarr. Brit. B. 2 edit. i. fig. p. 25.

Great Britain, Europe, N. America, India and the Coast of the Red Sea.

95. Pandion leucocephatus.

B. M.

Pandion leucocephalıs, Gould, B. of Austr. pl. 6. P. Gouldii, Kaup.

Australia. 
b. Ichthyaëtus, Lafr. 1839 .

Polioaètus, Kaup, 1847.

96. Pandion ichthyaetus.

B. M.

Falco icthyaëtus, Horsf. Res. Zool. pl. Haliaëtus plumbeus, Hodgs. H.ichthyaëtus, Cuv. Ichthyaëtus bicolor, G. R. Gr. I. hucarius, Hodgs. Pontoaëtus ichthyaëtus, G. R. Gr. Pandion ichthyaëtus, Vig. Pandion (Polioaëtus) ichthyaëtus, Kaup.

India and Malayan Arcbipelago.

97. Pandion humilis.

B. M.

Falco (Pandion) humilis, Temm. \& Müll. Verh, over de Natur. Gesch. de Nederl. t. 6. Ichtbyaëtus nanus, et Pontoaëtus nanus, $B$ l. Pandion (Ichthraëtus) humilis, et Pandion (Polioaëtus) humilis, Kaup.

India and Malayan Archipelago.

\section{Haliaetus.}

a. Haliaètus, Sav. 1809 .

98. Haliaktes atbicilla.

B. M.

Vultur alhicilla, L. Pl. enl. 112, 415. Falco ossifraga, et F. melanaëtos, $L$. F. albicaudus, $G m$. F. hinnularius, Lath. Aquila ossifraga, et A. albicilla, Pall. Su: Haliaëtus nisus, Sar. H. orientalis, H. borealis, H. islandicus, H. groenlandicus, et H. albicilla, Brchm. H. Pygargus, Eylon. Gould, B. of Eur. pl. 10. Kitul. Kupf. der Wog. t. 2, f. 2. Naum. Vög. t. 12, 13, 14. Yarr. Brit. B. 2 edit. i. fig. p. 20.

Great Britain, Europe, N. America, N. Africa and N.-E. Asia.

99. Haltaetus leccocephalus.

B. M.

Falco leucocephalus, L. Pl. enl. 411. Wils. Amer. Orn. pl. 36. F. pygargus, Daud. F. ossifragus, Wils. Amer. Orn. pl. 55, f. Aquila leucocephala, Pall. Sw. Haliaẽtus leucocephalus, Cux. Vieill. Ois. de TAmer. Sept. 1. 3. Gould, B. of Eur. pl. 11. Haliaêtus Washingtoni, Audub. B. of Amer. pl. 11. (q)

N. America and Norway. 
b. Thallasoaëtus, Kaup, 1844.

Thalassaëtus, Kaup, 1847.

Thalassiaëtus, Reichenb. 1850.

100. Haliaetus pelagicus.

Aquila pelagica, Pall. Falco imperator, Kitıl. Kupf. der Vög. t. 2, f. 1. F. leucopterus, Temm. Pl. col. 489. F. Stelleri, Tyzenh. Haliaëtus pelagicus, Temm. \&. Schl. Fauna Jap. t. 4, H. leucopterus, Less. Haliaëtus (Thalassaëtus) pelagicus. Kaup.

Siberia, Kamtschatka and Japan.

c. Cuncuma, Hodgs. 1837.

Pontoaëtus, Kaup, 1844.

Pontaëtus, Kaup, 1845.

Blagrus, $B l .1849$.

101. Haliaetus Macer.

B. M.

Falco Macei, Temm. Pl. col. 8, 223. Haliaëtus Macei, Cuv. H. fulviventer, Vieill. H. unicolor, Gr. Ill. Ind..Zool, pl. 19. H. lanceolatus, Hodgs. H. albicilla, Vig. \& Horsf. Pandion lineatus, Jerd.? Haliaëtus (Cuncuma) albipes, Hodgs. Ha. liaëtus (Pontoaëtus) Macei, Kaup. Ćuncuma Macei, G. $R$. $G r$.

India.

102 ? Haliaetus LEUCORYPHUS.

Aquila leucorypha, Pall. Haliaëtus leucoryphus, Keys. $\& \mathrm{Bl}$.

Caspian Sea and Ural Mountains.

103? HaliaEtus SINENSIS.

Falco sinensis, Gm. Lath. Gen. Syn. 1, pl. 3. Haliaëtus sinensis, G. R. Gr. H. Macei, juv. Kaup. Aquila sinensis, Steph. China.

104. Haliaetus leucogaster,

B. M.

Falco leucogaster, Gm. Pl. col. 49. F. blagrus. Daud. Levaill.

Ois. de l'Afr. t. 5. F. albicilla, var. Lath. F. dimidiatus, Raff. Haliaëtus blagrus, Cuv. H. leucogaster, Vig. H. sphenurus, Gould, B. of Austr. pl. 3. Ichthyaëtus cultrunguis, et Blagrus leucogaster, $B l$. Haliätus (Pontoaëtus) leucogaster, Kaup. Cuncuma leucogaster, G. R. Gr.

S. and W. Africa, India, Australia, Java, Sumatra and Celebes. 
105.? Haliatus maritimus.

Falco maritimus, Wurm. Haliaëtus maritimus, G.R.Gr. Java.

106. Haliaetus vocifer.

B. M.

Falco vocifer, Daud. Levaill. Ois. de l'Afr. t. 4. Aquila vocifer, Dum. Haliaëtus vocifer, Cuv. H. vociferoïdes, O. des Murs, Iconogr. Ornith. t. 17. Haliaëtus (Pontoaëtus) vocifer, Kaup, Cuncuma vocifer, G. R. Gr.

Greece, N.-E. and S. Africa.

d. Geranoaëtus, Kaup, 1844.

Heteroaëtus, Kaup, 1845.

Heteraëtus, Reichenb. 1850.

107. Haliaetus melanoleucus.

Spizaëtus melanoleucus, Vieill $\quad$ B. M. 302. Haliaëtus . melanoleucus, Less. Buteo aguia, Cab. Haliaëtus (Geranoaëtus) aguia, et H. (Heteroä̈tus) aguia, Kaup. Cuncuma melanoleuca, G. R. Gr. Geranoaëtus aguia, $\operatorname{Pr} . B$. Spizaëtus fuscescens, Vieill.

S. America (Brazil, Paraguay).

\section{e. Helotarsus, Smith, 1830. \\ Terathopius, Less. 1830.}

108. Haliaetus ecaudatus.

Falco ecaudatus, Daud. Levaill Ois. tarsus typus, $A$ Smith. Heloecaudatus, $C u v$. T. ecaudatus, G. R. Gr. Circaëtus N.-E. and S. Africa.

f. Haliastur, Selby, 1840.

Dentiger, Hodgs. 1844.

Ictinoaëtus, Kaup, 1844.

Milvaquila, Burm. 1850.

109. Haliaetus indus.

Falco indus, Bodd. $P l$ enl. 416. F ponticerianu, B. M.

. Fonticerianus, $\mathrm{Gm}$. $\mathrm{Ha}$ Cux. H. pondicerian. H. garuda, Less. H. ponticerianus, H. indus, G. R. G . Haliastur ponticerianus, Selby. dicaudus, India and Philippine Islands. 
110. Haltaetus leucosternus.

B. M.

Falco ponticerianus, Shaw, Nat. Misc. pl. 389. Haliaëtus leucosternon, Gould, B. of Austr. 1, pl. 4. Haliastur leucosternus, G. R. Gr. Haliaëtus (Ictinoaëtus) leucosternon, Kaup.

N. Australia and New South Wales.

111. Haliaetus sphenurus.

B. M.

Milvus sphenurus, Vieill. Gal. des Ois. t. 15, Sw. Haliaëtus canorus. Vig. \& Horsf. Haliastur sphenurus, Gould, B. of Austr. 1, pl. 5. Haliaëtus (Ictinoaëtus) canorus, Kaup.

Australia (except V. D.'s Land.)

\section{iv. Falconin}

Falconinæ, Sw. 1837, p ; Pr. B. 1831-38-49-50; G. R. Gr. $1840-41-44 ;$ Kaup, $1847 ; B l .1849$.

Falconina et Accipitrina, p. Vig. 1825.

Falcones, Kaup, 1844.

\section{Falco.}

a. Falco, L. 1735 .

Rhynchodon, Nitzsch, 1840.

Ichthierax, Kaup, 1844.

Hierofalco, Cuv. 1817.

112. Falco candicans.

B. M.

Falco candicans, Gm. Pl. enl. 446. F. grœnlandicus, Brehm. Hanc. Gould, B. of Eur. 1, pl. 19. Hierofalco candicans, Cuv. H. gyrfalco, Eyton. H. gronlandicus, Brehm. Gytfalco candicans, Flem. Yarr. Brit. B. 2 edit. i. fig. p. 31. Falco (Hierofalco) candicans, Kaup. Falco islandicus, Brünn. Hanc. F. gyrfalco, Keys \& Bl. F. candicans, islandicus, Schl. Hierofalco islandicus, Brehm. Falco (Hierofalco) islandicus, Kaup. Falco gyrfalco $(L !), S c h l$. F. laniarius, $\boldsymbol{L}$. Pl. enl. 462. Falco (Hierofalco) gyrfalco, Kaup.

Great Britain, N. Europe, Greenland, Iceland and N. America.

113. Falco subigiger.

B. M.

Falco subniger, G. R. Gr. Gould, B. of Austr. 1, pl.9. Falco (Hierofalco) subuiger, Kaup.

S. Australia. 
114. Falco peregrinator. B. M.

Falco peregrinator, Sundev. F. shaheen, Jerd. Ill. Ind. Orn. pl. 12. F. sultaneus, Hodgs. F. rhombeus, Lath?

India, (Nepal).

115. FALCO PEREgrinus.

B. M.

Falco peregrinus, Gm. $P l$. enl. 430, 421, 470, 469. F. barbarus, $L$. F. abietinus, Bechst. F. punicus, Levaill. F. micrurus, Hodgs. F.calidus, Lath. F. cornicum, Brehm. F. communis, Gm. Schl. F. peregrinoïdes, v. d. Mulhe. F. minor, Pr. B. F. peregrinus, minor, Schl. The Lanner, Penn. F. orientalis, Gm. Sparvius orientalis, Vieill. Gould, B. of Eur. pl. 21. Yarr. Brit. B. 2 edit. i. fig. p. 37.

Great Britain, Europe, India and Africa.

116 ? Falco anatum.

B. M.

Falco peregrinus, Wils. Amer. Orn. pl. 76. F. anatum, $\operatorname{Pr} . \stackrel{B}{B}$.

$\mathrm{N}$. America and $\mathrm{W}$. Indies.

117 ? Falco melanogenYs.

B. 11 .

Falco peregrinus, Vig. \& Horsf. F. melanogenys, Gould. F. macropus, Sw. F. peregrinoides? Peale. Gould, B. of Austr. pl. 8 .

Australia.

118. Falco peregrinoides.

B. M.

Falco peregrinoïdes, Temm. $P l$. col. 479. F. frontalis, Daud? Levaill. de l'Afr. t. 28? F. galericulatus, Shaw.? F. (Ichthierax) frontalis, Kaup?

N. E. and S. Africa.

b. Gennaia, Kaup, 1847.

119. Falco lanarius.

B. M.

Falco lanarius $(L$ ? $)$ Pall. $P l$. enl. 470. F. Feldeggii, Schl.

F. sacer, Gm. Schl. F. cyanopus, Tiedem. F. cherrug, Gr. Ill.

Ind: Zool. pl. 25. Falco (Gennaia) lanarius, Kaup. Naum.

V̈̈g. t. 23. Gould, B. of Eur. 1, pl, 20.

E. Europe and N. India. 
120. Falco tanypterus.

Falco tanypterus, Licht. Schl. Abhand.t. 12, 13. Falco(Gennaia) tanypterus, Kaup.

N. E. Africa and Dalmatia.

121. Falco hypoleucus.

Falco hypoleucos, Gould, B. of Austr. 1, pl. 7. Falco (Gennaia) hypoleucus, Kaup.

S. and W. Australia.

122. Falco cervicalis.

B. MI.

Falco cervicalis, Licht. F. biarmicus, Temm. Pl. col. 324. F. chicqueroïdes, A. Smith. Falco (Gennaia) cervicalis, Kaup.

S. and N.-E. Africa.

123. Falco jugGer.

B. M

Falco jugger, $G r$. Ill. Ind. Zool. pl. 26. F. lugger, Jerd. F. thermophilus, Hodgs. Falco (Gennaia) jugger, Kaup.

N. India.

c. Hypotriorchis, Boie, 1826.

Dendrofalco, G. R. Gr. 1840.

124. Falco subbuteo.

B. $\mathrm{M}$.

Falco subbuteo, L. Pl. enl. 432. F. barletta, Daud. F. subbuteo, major, Lath. F. pinetarius, Shaw. F. hirundinum, Brehm. Hypotriorchis subbuteo, Boie. Falco (Hypotriorchis) subbuteo, Kaup. Gould, B. of Eur. pl. 22. Yarr. Brit. B. 2 edit. i. fig. p. 45.

Great Britain, Europe, Asia and Africa.

125. Falco ELEONORE.

Falco eleonoræ, Gené, Mem. Acad. Torina, 1840, t. 1, 2 T. concolor, Temm. et $v$. d. Mulhe. F. arcadicus, Linderm. Isis, 1843, t. 1. Falco (Hypotriorchis) eleonoræ, Kaup. Hypotriorchis eleonoræ, G. R. Gr. Pr. B. Fauna Ital. Aves, t. 1.

Sardinia and Greece.

126. FAlco severus.

B. M.

Falco severus, Horsf. F.Aldrovandi, Reinw. Pl. col. 128. F. rufipedoïes, M'Clell. F. guttatus, G. R. Gr. F. religiosa, Temm. Hypotriorchis severus, G. R. Gr. Falco (Hypotriorchis) severus, Kaup.

N. Asia, Java and Philippine Islands. 
12\%. Falco Rufigularis.

B. M.

Falco rufigularis, Daud. F. aurantius, var. Lath. F. cucullatus, Sw. P. thoracicus, Donov. F. deirolencus, Temm. Pl. col. 348. Hypotriorchis rufigularis, G. R. Gr. Falco (Hypotriorchis) aurantius, Kaup. Penn. Gen. of B. pl. 1.

S. America, (Brazil, Bahia, Guiana).

128. Falco frontatus.

B. M.

Falco frontatus, Gould, B. of Austr. 1, pl. 10. F. lunulatus, Lath.? F. longipennis, Sw. F. subbuteo, Brehm. Sparvius lunulatus, Vieill.? Hypotriorchis frontatus, G. R. Gr. Falco (Hypotriorchis) frontatus, Kaup.

Australia.

$$
\text { d. Esalon, Kaup, } 1829 .
$$

129. Falco ÆSALON.

Falco asalon et $\mathrm{F}$. cæsius, Meyer. F. lithofalco, Gm. Pl. enl. 447, 468. F. cæsins, Meyer. F. regulus, Pall. F. sibiricus, Shaw. F. smirillus, Sav. Esalon lithofalco, Kaup. Falco (Æsalon) li. thofalco, Kaup. Hypotriorchis æsalon, G. R. Gr. Pall.Zoogr. t. 7. Gould, B of Eur. pl. 24. Yarr. Brit. B. 2 edit. i. fig. p. 53 .

Great Britain, Europe and N.-E. Africa.

130. Falco tibialis.

Falco tibialis, Daud Levaill Ois de $\mathrm{Afr}$. 29. F. B. Temm. Pl col 330. F ardosiacus, Vicill. F. F. concolor, $B$. of P. col. 330 . F. ardosiacus, Vieill. F. unicolor, $S w$. B. of $W . A f r .1$, pl. 3. Hypotriorchis tibialis et $\mathrm{H}$. concolor, G. R. Gr. H. ardesiacus, $\operatorname{Pr}$. B. Falco (Esalon) tibialis et F. (Æsalon) concolor, Kaup. Tinnunculus concolor, Rüpp. Gould, B. of Eur. 1, pl.25.

W. and N.-E. Africa and the coast of the Mediterranean Sea.

131. Falco columbarius.

Falco columbarius, $L$ Wils. temerarius, Audub, F, intermixtus, Bosc. pl. 15, f. 3. F, Rich. Fauna Bor-Amer. pl. 25. Tinnunculus columbarius, Vieill. Ois. de TAmer. Sept. t. 11. Falco et Nisus columbarius, Cuv. Astur columbarius, Boie. Hypotriorchis columbarius, G.R. Gr. Falco (Esalun) columbarins, Kaup.

$N$. America and W. Indies. 
132. Falco femoralis.

B. M .

Falco femoralis, Temm. Pl. col. 121,343. F. aurantius, var. $\gamma$. Lath. F. thoracicus, Ill. F. cyanescens, Vieill. F. elegans, Sw.MSS. F. hæmorrhoidalis, Hahn. Bidens femoralis, Spix. Hypotriorchis femoralis, Boie. Falco (Esalon) femoralis, Kaup.

S. America, (Mexico, Brazil and E Patagonia).

133. Falco chicquera.

B. $M$.

Falco chicquera, Daud. Levaill. Ois. d.Afr. t. 30. F. ruficollis et F. macrodactylus, $S w$. B. of $W$. Afr. pl. 2. F. cirrhata, var. Lath. Hypotriorchis chicquera, G. R. Gr. Falco (Esalon) chicquera, Kaup. Gould, Cent. of B. pl. 20.

India, N.-E. S. and W. Africa.

\section{e. Jeracidea, Gould, 1837.}

Hieracidea, Riechenb. 1850.

134. Falco Berigora.

B. $M$.

Falco berigora, Vig. \& Horsf. Jeracidea berigora et J. occidentalis, Gould, B. of Austr. 1, pl. 11, 12.

New South Wales, S. and W. Australia, Van Diemen's Land.

135. Falco Nover SeElandia.

Falco novæ seelandiæ, Gm. F. brunneus, Gould, F. harpe, Forst. F. australis, Homb. \& Jacq. Voy. au Pole Sud, Ois. t. 1, f. 1. F. ferox, Peale, U. S. Explor. Exped. pl. 18. Hypotriorchis novæ zealandiæ, $G$. R. Gr. Jeracidea novæ zealandiæ, Kaup.

New Zealand and Auckland Islands.

\section{f. Tinnunculus, Vieill. 1807.}

Cerchneis, Boie, 1826.

Falcula, Hodgs. 1837.

Egypius, Kaup, 1829.

136. Falco tinnunculus.

Falco tinnunculus, L. Pl. enl.401, 471. F. brunneus, Bechst. F. fasciatus, Retz. F. rufescens, $S w$. F. interstinctus, $\boldsymbol{M}$ Clell. F. tinnunculus, japonicus, Temm. \& Schl. Fauna Jap. t. 1, B. Tinnunculus alaudarius et T. interstinctus, G. R. Gr. Cerchneis tinnuncula, Boie, Brehm. C. murorum et C. media, Brehm. Egspius tinnunculus, Kaup. Gould, B. of Eur. pl.

26. Yarr. Brit. B. 2 edit. i., fig. p. 57.

Great Britain, Europe, India, Japan, N. and W. Africa. 
137. Falco moldccensis.

Tinnunculus moluccensis, Schl. Homb. \& Jacq. Voy. au Pole Sud, t. 1, f. 2.

Malayan Peninsula.

138. FALCO RUPICOLUS.

B. M.

Falco rupicolus, Daud. Levaill. Ois. de l'Afr.t. 35. F. capensis, Shaw. Cerchneis rupicola et C. capensis, Boie. Tinnunculus rupicolus, G. $R$. $G r$.

S. Africa.

139. Fatco rupicoloides.

B. M.

Falco rupicoloïdes, A. Smith, Ill. S. Afr. Zool. pl. 92. Tinnunculus rupicoloïdes, $G . R$. $G r$.

S. Africa.

140. Falco punctatus.

B. M.

Falco punctatus, Cuv. Pl. col. 45. Cerchneis punctatus, Boie. Tinnunculus punctatus, G. R. Gr.

Madagascar.

141 Falco gracilis.

Falco gracilis, Less. O. des Murs, Iconogr. t. Tinnunculus gracilis, G. $R . G r$.

Seychelle Island.

142. Falco Cenchroides.

B. M.

Falco cenchrö̈des, Vig. \&.Horsf. Gould, B. of Austr. 1, pl. 13.

Cerchneis immaculatus, $B$ rehm. Tinnunculus cenchroïdes, $G$. R. $G r$.

Australia (except Van Diemen's Land.)

g. Tichornis, Kaup, 1844.

143. Falco cenchris.

B. M.

Falco cenchris, Naum. Vög. Nacht. t. 29. F. tinnunculoides, Schinz. F. tinnuncularius, Vieill. F. xanthonyx, Natt. F. Naumanni, Fleisch. Agypius, cenchris, Kaup. Cerchneis cenchris, Boie. Tinnunculus cenchris, $\operatorname{Pr}$. B. Tinnunculus (Tichornis) cenchris, Kaup. Voy. de la Morea, Ois. t. 2, 3. Gould, B. of Eur. pl. 27.

E. Europe and N.-E. Africa. 


\section{h. Erythropus, Boie, 1828. Pannychistes, Kaup, 1829.}

144. Falco vespertinus.

B. M.

Falco vespertinus, $L . P l$. enl. 431 . F. rufipes, Besele. Tinnunculus vespertinus, $G$. $R$. Gr. Cerchneis vespertinus, Boie. Erythropus vespertinus, Brehm. Pannychistes rufipes, Kaup. Falco (Erythropus) rufipes et F. (E.) vespertinus, Kaup. Gould, B. of Eur. pl.23. Kittl. Kupf. der Vög. t. 3, f. 1. Pall. Zoogr. t. 6. Yarr. Brit. B. 2 edit. i. fig. p. 49.

Great Britain, Europe, Greece, N.-E. Africa, and Nepal.

\section{i. Pœcilornis, Kaup, 1844.}

145. Falco sparverius.

B. $\mathrm{M}$.

Faleo sparverius, $L$. Pl. enl. 444, 465. Wils. Amer. Orn. pl. 32, f. 1, pl. 16, f. 1. F. dominicensis, Gm.? F. gracilis et F. isabellinus, Sw.? Bidens sparverius, Spix.? Cerchneis sparveritus, Boie. Tinnunculus sparverius, Vieill. Tinnunculus (Pœcilornis) sparverius, Kaup.

N. and S. America.

146. Falco sparveroides.

Falco sparveroïdes, Vig. Fras. Zool. Typ. pl. Voy. l'isl. Cuba, Ois. t. 1. Tinnunculus sparveroïdes, G. R. Gr. T. (Pœcilornis) sparveroïdes, Kaup.

Cuba.

147. Falco cinnamomines.

B. M.

Falco cinnamominus, $S w$. Tinnunculus sparverius, Vieill. Ois. de l'Amer. Sept.t. 12, 13? T. cinnamomeus, G. R. Gr. Southern parts of S. America.

\section{j. Polihierax et Poliohierax, Kaup, 1847.}

148. Falco semitorquatus.

Falco semitorquatus, A. Smith, Ill. S. Afr. Zool.t. 1. Tinnunculus semitorquatus, G. R. Gr. 'Tinnunculus (Polihierax) semitorquatus, Kaup. Polihierax semitorquatus, $\operatorname{Pr} . B$.

S. Africa. 


\section{Harpagus.}

Harpagus, Vigors, 1824.

Bidens, Spix, 1824.

Diodon, Less. 1831.

Diplodon, Nitzsch, 1840.

149. Harpagus diodon.

Falco diodon Temm Bidens femoralis et $\mathbf{B}$. cinerascens, Spix, Av. Bras. t. 8. Diodon brasiliensis, p. Less. Harpagus diodon, Vigors.

S. America, (Brazil).

150. Harpagus bidentatus.

B. M.

Falco bidentatus, Lath. $\mathrm{Pl}$. col. 38, 228. Bidens rufiventer et B. albiventer, Spix, Av. Bras. t. 6, 7. Diodon brasiliensis, p. Less. Harpagus bidentatus, Vigors.

S. America, (Guiana, Brazil).

\section{IERAx.}

Ierax, Vigors, 1824.

Hierax, Cuv. 1829.

151. Ierax carolescens. B. $M$.

Falco cærulescens, L. Edw. B. pl. 108. F. fringillarius, Drap. Jerax cærulescens, Vigors. J. malayensis, Strickl. Hierax fringillarius, $\boldsymbol{B l}$.

Java, Borneo.

152. IERAX BENGaLENSIS.

B. M. Jerax eutolmus, Hodgs. Falco bengalensis, Bl. Jerax erythrogenys, Vig. J. bengalensis, $\mathrm{Pr}$. B. Hierax eutolmus, $B l$. H. melanoleucos, $B l$.

India (Nepal, Tanesserim).

153. Ierax sericeus.

B. M.

Falco sericeus, Kittl. Mem. de l'Acad. Imp. St. Petersb. (1835) t. 1. Kittl. Kupf. der Vög. t. 3, f. 3. F. Gironnierii, Eyd. et Souley, Voy. de la Bonite, Ois. t. 1. Ierax sericeus, G. R. Gr.

China and Philippine Islands. 


\section{v. Miluinæ.}

Milvinx, Pr. B. 1838-49-50; G. R. Gr. 1840-41-45.

Milvina, Vigors, 1825.

Aquilina, p., Accipitrina, p. et Buteonina, p., Vigors, 1825.

Falconinæ, p. et Buteoninæ, p., Pr. B. 1838.

Cymindinæ, p., Buteoninæ, p. et Accipitrinæ, p., $S w .1837$.

Circi, p. et Buteones, p. Kaup, 1844.

Buteoninæ, Kaup, 1847.

Perninæ, Elaninæ et Haliaëtinæ, p., Bl. 1849.

\section{BAZA.}

a. Baza, Hodgs. 1836.

Lophotes, Less. 1831.

Lepidogenys, Gr. 1839.

Hyptiopus, Hodgs. 1841.

Lophastur, $B l .1842$.

Aviceda, p. $\operatorname{Pr}$. B. 1850.

154. BAZA LOPHOTES.

B. M.

Falco lophotes, Cuv. Temm. Pl. col. 10. F. Lathami et Lepidogenys Lathami, Gr. Buteo cristatus, Vieill. Lophotes indicus, Less. Baza syama, Hodgs. B. lophotes, G. R. Gr. Pernis (Hyptiopus) lophotes, Kaup. Aviceda lophotes, Pr. B. India.

155. Baza sUbCristata.

B. M.

Lepidogenys subcristatus, Gould, B. of Austr. 1, pl. 25. Baza subcristata, G.R. Gr. Aviceda subcristata, Pr. B. Pernis (Hyptiopus) subcristatus, Kaup.

New South Wales and N. Australia.

156. BAZA JERDONI.

Pernis (Lophastur) Jerdoni, $B l$. Baza Jerdoni, Bl. G. R. Gr.

"? Falco Reinwardtii, Mill." Bl. Aviceda lophotes? $\operatorname{Pr}$. B.

Malacea.

157. BazA Magnirostris.

B. M.

Baza magnirostris, G. R. Gr. Aviceda magnirostris, Pr. $B$. Pernis (Hyptiopus) magnirostris, Kaup.

Philippine Islands. 
158. Baza Reinwardit.

Falco (Lophotes) Reinwardtii, Miull. \& Schl. Verh. Nederl. t. 5. Aviceda Reinwardtii, $\operatorname{Pr}$. B. Baza Reinwardtii, G. R. Gr. Pernis (Hyptiopus) Reinwardtii, Kaup.

Celebes.

159 ? Baza SUMATRENSIS.

Aviceda sumatrensis, Lafr. Baza sumatrensis, G.R. Gr. Aviceda Reinwardtii, p. Pr. $B$.

Sumatra.

$$
\begin{aligned}
& \text { b. Aviceda, Sw. } 1837 . \\
& \text { Ichthierax (Kaup), Pr. B. } 1850 .
\end{aligned}
$$

160. Baza cuculordes.

B. M.

Aviceda cuculoïdes, $S w$. B. of W. Afr. pl. 1. A. Verreauxii et

A. buteoïdes, Lafr. Aviceda frontalis (Daud.? $) \operatorname{Pr} . B$.

W. and S. Africa.

\section{Pernis.}

Pernis, Cuv. 1817.

161. Pernis apivorus.

B. M.

Falco apivorus, L. Pl. enl. 420. F. poliorhynchus, Bechst. F. dubius, Sparr. F. incertus, Lath. Accipiter lacertarius, Pall. Buteo apivorus, Vieill. Pernis apivorus, $C u v$. P. apium et P. vesparum, Brehm. P. communis, Less. Gould, B. of Eur. pl. 16. Jard. \& Selby, Illustr. Ornith. n. s. pl. 1. Yarr. Brit. B. 2 edit. i. fig. p. 91.

Great Britain, Europe, Siberia, Japan, W. and N..E. Africa.

162 ? Pernis tachardes.

Falco tachardus, Daud. Levaill. Ois. de l'Afr.t. 19. Buteo tachardus, Vieill. Pr. B. B. capensis, p., Temm. \& Schl. Pernis madagascariensis, $A$. Smith?

S. Africa, Madagascar?

163. Pernis cristata.

B. $M$.

Pernis cristata, Cuv. Reg. An. t. 3, f. 4. P. Elliottii, James. P. bharatensis, v. apivorus, Hodgs. P. maculosa, P. torquatus, $\mathrm{P}$. ruficollis et P. albigularis, Less. Falco ptilorhynchus, Temm. Pl.col. 44. Verh. over de Natur. Gesch. de Nederl. Ois. t. 7 .

India and Malayan Archipelago. 


\section{Cymindis.}

a. Cymindis, Cuv. 1817.

Leptodon, Sundev. 1836.

Odontotriorchis, Kaup, 1844.

164. Cymindis Cayanensis.

B. $M$.

Falco cayanensis, Gm. Pl. col. 270. F. palliatus, Pr. Max.

Pl.'col. 204. Spix, Av. Bras. t. 8, c. Cymindis cayanensis, Cuv. C. buteonides, Less. Asturina cyanopus, Vieill. Mag. de Zool. 1835. Ois. t. 22. Sparvius monachus, S. cinereus, S. bicolor, et S. griseus, Vieill. Cymindis (Odontriorchis) cayanensis, Kaup. Pernis (Odontriorchis) cayanensis, Kaup.

S. America (Brazil, Guiana).

b. Regerhinus, Kaup, 1845.

165. Cymindis uncinatus.

B. $M$.

Falco uncinatus, Illig. $P l$. col. $103,104,115$. F. vitticaudus, Pr. Max. Cymindis uncinatus, Cuv. C. cuculoïdes, Sw. Pernis (Regerhinus) uncinatus, Kaup. Mag. de Zool. 1835. Ois. t. 21.

S. America (Guiana, Brazil).

166. Crmindis Wilsoni.

Cymindis Wilsoni, Cass. Journ. Acad. N. S. Philad. 1847, pl. 7.

Cuba.

\section{Milvos.}

a. Milvus, Cuv. 1799-1800.

167. Milvus Regalis.

B. $\mathrm{M}$.

Falco Milrus, $L . P l$. enl. 422. F. austriacus, $G m$. Accipiter korschun, S. G. Gm. A. regalis, Pall. Milvus regalis, Briss. M. ictinus, Sav. M. castaneus et M. russicus, Daud. M. jaicensis, Lepech. M. vulgaris, Flem. M. ruber, Brehm. Gould, B. of Eur. pl. 28. Yarr. Brit. B. 2 edit. i. fig. p. 71.

Great Britain; Europe and N. Africa. 
b. Hydroictinia, Kaup, 1844.

168. Milvus ater.

B. M.

Falco ater, $\mathrm{Gm}$. $\quad \mathrm{Pl}$. enl. 472. F. cinereo-ferrugineus, Forst. F. migrans, Bodd. F. fusco-ater, Meyer. Milvus ater, Daud. M. œtolius, Sav. M. fuscus, Brehm. M. niger, $\operatorname{Pr} . B$. Accipiter milvus, Pall. Milvus (Hydroictinia) ater, Kaup. Gould, B. of Eur. pl. 29.

Europe, Asia, (temperate parts).

169 Milvus govinda.

Milrus govinda, Sykes $\mathrm{M}$. \&. Scht Fom Jap. 9. Schl. Fauna Jap. t. 5. M. indicus, v. cheela, Hodgs. M. ater, Bl. Haliaëtus lineatus, $G r$. Ill. Ind. Zool. pl. 18. Milvus (Hydroictinia) govinda, Kaup. Fauna Jap. t. $5, \boldsymbol{B}$.

India and Japan.

170. Milves affinis. B. M. Milvus affinis, Gould B. of Austr. pl. 21. M. ater? Bl. Milrus (Hydroictinia) aflinis, Kaup.

Anstralia.

171. Milvus eggrties.

Falco ægyptius et F. Forskallii, Gm. F. parasitus, Daud. $\quad$ F. parasiticus, Lath. Levaill. Ois. de l'Afr. t. 22. Milvus ægyptius, G. R. Gr. M. parasitus, $P r . B$. M. parasiticus, Less. Milvus (Hydroictinia) parasiticus, Kaup. Disc. de l'Egypte, Ois. t. 3, t. 1.

Dalmatia ; W., N. and S. Africa.

c. Lophoictinia, Kaup, 1847.

172. Milves isurus.

Milrus isurus, Gould, B. of Austr. i. pl.22. Falco pacificus Lath.? Milvus (Lophoictinia) isurus, Kaup.

New South Wales and S. Australia. 


\section{Elanus.}

a. Elanus, Sav. 1809.

Elanoïdes, Vieill. 1818.

173. Elands melanopterus.

B. $\mathrm{M}$.

Falco melanopterus, Daud. Levaill. Ois, de l'Afr. t. 36, 37. F. sonninensis et F. vocifer, Lath. F. clamosus, Shaw. Buteo vocifer, Vieill. Elanus melanopterus, Leach, Zool. Misc. pl. 122. E. cæsius, Sav. E. minor, $\operatorname{Pr}$. B.? Elanoïdes cæsius, Vieill. Gould, B. of Eur. pl. 31. Disc. de lEgypte, Ois. t. 2, f. 2 .

Europe, Africa, India and its Archipelago.

174. Elandes axilla Ris.

Falco axillaris, Lath. Gould, B. of Austr. i. pl. 23. Elanus melanopterus, Vig.\&. Horsf. E. notatus, Gould. E. axillaris, G. R. Gr. Circus axillatis, Vieill.

Australia (except the North).

175. Elanus scriptus,

B. M.

Elanus scriptus, Gould, B. of Austr. i. pl. 24.

S. Australia.

176. Elanus letcurus.

B. M.

Milvus leucurus, Vieill. Falco dispar, Temm. Pl. col. 319. F. melanopterus, $\mathrm{Pr}$. B. Amer. Orn. pl. 11, f. 1. Elanus dispar, Cuv. E.leucurus, G. R. Gr. Elanoïdes leucurus, Vieill. Southern parts of N. America; S. America, (Paraguay, Brazil, Bahia).

b. Gampsonyx, Vigors, 1825.

177. Eianus Swatssoni.

B. M.

Gampsonyx Swainsoni, Vigors. Gr. \& Mitch. Gen. of B. pl. G. Holmii, Blackw.? Elanus torquatus, Cuv. Falco rufifrons, Pr. Max. Nertus rufifrons, Boie. Elanus (Gampsonyx) Swainsoni, Kaup.

S. America, (Brazil). 


\section{Nauclerus.}

a. Nauclerus, Vigors, 1825.

Elanoïdes. G. R. Gr.

178. Nauclenus Furcatus.

B. M.

Falco furcatus, L. Wils. Amer. Orn.pl. 51, f. 2. Gould, B. of Eur. pl. 30. Shaw, Nat. Misc. pl. 204. Elanus furcatus, Cuv. Elanoides furcatus et E. yetapa, Vieill. Nauclerus furcatus, Vigors. Milvus furcatus, Viéill. Ois. de l'Amer. Sept. t. 10. Yarr. Brit. B. 2 edit. i. fig. p. 77.

England! S. America and Southern parts of N. America.

b. Chelidopteryx, Kaup, 1843.

Chelictinia, Less. 1843.

179. Nauclerus Riocouri.

B. M.

Elanoïdes Riocouri, Vieill. Gal. des Ois. t. 16. Falco Riocuurii, $P$. col. 85. Elanus Riocouri, Cuv. Nauclerus Riocouri, Vigors. Nauclerus africanus, Sw. Chelictinia Riocourii, Less. Nauclerus (Chelidopteryx) Riocouri, Kaup.

W. Africa.

\section{ICTINIA.}

a. Ictinia, Vieill. 1816.

180. ICTINIA MISSISSIPPENSIS.

B. M.

Falco mississippensis, Wils. Amer. Orn. pl. 25, f. 1. Milvus mississippensis, Cuv. Ictinia mississippensis, Kaup. I. ophiophaga, Vieill. Nertus mississippensis, Boie.

Southern parts of N. America.

b. Nertus, Boie, 1828.

Pœeilopteryx, Kaup, 1843.

181. ICtinia Plumbea.

B. M.

Falco plumbea, $G m$. $P l$. col. 180. Ictinea plumbea, Vieill. Milvus cenchris, Vieill. Ois. de l'Amer. Sept. t. 10, bis. Nertus plumbeus, Boie. Buteo plumbeus, Cuv. Ictinia (Pcecilopteryx) plumbea, Kaup. Spix, Av. Bras. t. 8.

South America, (Brazil, Guiana) and Mexico. 


\section{Rostrhamus.}

Rostrhamus, Less, 1831.

182. Rostrhamus soctabilis.

B. M.

Herpetotheres sociabilis, Vieill. Falco hamatus, Illig. Pl.col. 61,231. F. leucopygus, Spix, Av. Bras. t. 2. Buteo hamatus, Vieill. Cymindis hamatus, Cuv. Rostrhamus sociabilis, D'Orb. \&. Lafr. R. hamatus, G. R. Gr. R. niger, Less. Mag. Zool. (1834) Ois. t. 20.

S. America, (Brazil).

vi. ACCIPITRINE,

Accipitrinæ, Sw. 1837; Pr.B. 1838-49-50; G. R. Gr. 1840$4 \mathrm{I}-49 ;$ Kaup, $1847 ; B l .1849$.

Accipitrina, Vigors, 1825.

Falconinæ, p. $\operatorname{Pr}$. B. 1831.

Circinæ, p. Bl. 1849.

\section{Astur.}

a. Astur, Lacep. 1800-01.

Dædalion, Sav. 1809.

Sparvius, Vieill. 1816.

183. Astur paldMbariUs.

B. MI

Falco palımbarius, $L$. $\quad P l$. enl. $418,461,423$. F. gentilis, $L$.

F. gallinarius, Gm. F. albescens, Bodd. Astur palumbarius,

Bechst. A. gallinarum, Brehm. Dædalion palumbarius, Sav.

Accipiter astur, Pall. Zoogr. t. 11. A. palumbarius, Jenyns. Buteo palumbarius, Flem. B. gallinarius, Bechst. Gould, B. of Eur. i. pl. 17. Yarr. Brit. B. 2 edit. i. fig. p. 62.

Great Britain, Europe, Nepal and N.-E. Africa.

184. Astur atricapillus.

B. M.

Falco atricapillus, Wils. Amer. Orn. pl. 52, f. 3. F. regalis, Temm. Pl. col. 495. Sparvius atricapillus, Vieill. Drdalion pictum, Less, Astur palumbarius, p. Kaup. Fauna Bor.Amer. Birds, pl. 26. Pr. B. Amer. Orn. pl. 10, f. 1. Jard. \&. Selby, Ill. Ornith, pl. 121.

N. America. 
185. Astur melanoleucus.

B. M.

Astur melanoleucus, A. Smith, Ill. S. Afr. Zool. pl. 18. A. Smithi, Kaup. Accipiter melanoleucus, A. Smith.

S. Africa.

\section{b. Spizageranus, Kaup, 1845 .}

186. Astur unicinctus.

B. M.

Falco unicinctus, Temm. Pl. col. 313. F. anthracinus, Licht.

Nisus unicinctus, Less. Astur unicinctus, Cuv. Buteo Harrisii, $A u b u b . B$. of Amer. pl. 392. B. unicinctus, G. R. Gr. Polyborus tæniurus, Cab. Tschudi, Fauna Per. t. 1. Hypomorphnus unicinctus, $C a b$. Asturina (Spizageranus) unicinctus, Kaup.

S. America, (Brazil, Peru, Chili) and Mexico (?)

$$
\text { c. Leucospiza, Kaup, } 1844 .
$$

187. Astur Novæ hollandiæ.

B. M.

Falco novæ hollandiæ, $\mathrm{Gm}$. F. albus, Shaw. F. lencaëtos, Forst. F. clarus, Lath. Astur novæ hollandiæ, Cuv. A. albus, Sw. A. Rayii, Vig. \& Horsf. Sparvius niveus, Vieill. Dædalion candiclum, Less. Astur (Leucospiza) novæ hollandiæ, Kaup. Jard. \& Selby, Illustr. Orn. pl. 1. Gould, B. of Austr. pl. White's Bot. Bay, pl. p.

New South Wales; S. and N. Australia.

\section{d. Lophospiza, Kaup, 1844.}

188. Astur trivirgatus.

Falco trivirgatus, Temm. Pl. col. 303. F. anceps, Temm. Astur trivirgatus, Cuv. A. cristatus, G. R. Gr. A. palumbarius, Jerd. A. indicus, Hodgs. Spizaëtus rufistinctus, $M$ 'Clell. Astur (Lophospiza) trivirgatus, Kaup. Dædalion trivirgatum, Less.

W. India, Philippine Islands and Sumatra.

189. AStur GRISEICEPS.

Astur griseiceps, Temm. Astur trivirgatus, p.? Pr. B. Astur (Lophospiza) griseiceps, Kaup.

Cielebes. 
190. Astur trinotatus.

Astur trinotatus, Temm. Accipiter trinotatus, Pr. B. Consp. p. 33. Astur (Lophospiza) trinotatus, Kaup.

Celebes.

191. Astur longichudus.

Falco longicaudus, Garn. Voy. de la Coqu. t. 10. Dædalion longicauda, Less.

New Guinea.

\section{e. Asturina, Vieill. 1816.} Rupornis, Kaup, 1844.

192 Astur Nitidus.

Falco nitidus, Lath. F. striolus, B. M.

Dædalion nitidus, Temm. Pl. col. 87, 294. Cuv. Asturin Less. Astur nitidus, Steph. A. striolatus, Kaup.

S. America, (Brazil, Bahia, Caraccas, Guiana) aud Mexico.

193. Astur poliogaster.

Falco poliogaster, Temm Pl B. M. Cuv. Nisus pileatus, Tschudi. $_{\text {. }}$. 264,295 . Astur poliogaster, rina poliogaster, Kaup.

S. America, (Brazil, Peru).

194. Astur leucorrhous.

Falco leucolrhous, Quoy \& Gaim. Voy. de l'Uranie, t. 13. Astur lencorrhous, $C u v$. Nisus leucorrhöus, $C a b$. Dædalion leucorrhous, Less. Asturina leucorrhous, Kaup.

S. America, (Caraccas).

\section{Astur magnirostris.}

Falco magnirostris, $G m$. $P l$ B. M.

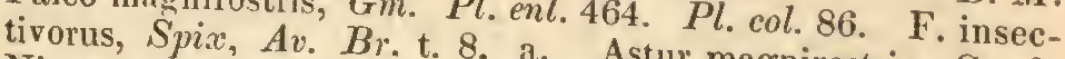
Nisus magnirostris, Cuv. Asturina Astur magnirostris, Steph. turina (Rupornis) magnirostris, Kaup.

S. America, (Guiana, Brazil, Caraccas, Bolivia).

196. Astur albifrons.

Asturina albifrous, $K a u p, 1$ sis, 1847, t. f. Astur albifrons, $G$.
$R$. $G$.

S. America. 
Examine, Astur rufitorques, Peale, U. S. Expl. Expd. viii. p. 68, pl. 19.

Feejee Islands.

Astur — ? Griff. An. Kingd. i. pl. p. 236.

f. Melierax, G. R. Gr. 1840.

197. Astur canorus.

B. M

Falco canorus, Thunb. F. musicus, Daud. Levaill. Ois. de l'Afr. t. 27. F. polyzonus, Rüpp. Fauna Abyss. t. 15, f. 1 . Nisus musicus, Cuv. N. canorus, Less. Melierax musicus, G. R. Gr. Pr. B. M. polyborus, $P r . B$. Astur musicus, $S w$. Less. Nisus (Melierax) musicus, Kaup. Astur (Melierax) cantans, Kaup. Accipiter musicus, Vig.

S. and N-E. Africa.

g. Micronisus, G. R. Gr. 1840.

Nisastur, $B l .1844$.

198. Astur sphenurus.

B. $\mathrm{M}$.

Falco sphenurus, Rüpp. Syst. Uebers. von Nord-Ost-Afr. t. 2.

Micronisus sphenurus, G. R. Gr. Accipiter brachydactylus, $S w$. A. polyzonoïdes, $A$. Smith, Ill. S. Afr. Zool. pl. 11. A. sphenurts, G. R. Gr. Nisus sphenurus, Rüp. Astur (Micronisus) Ruppellii, Kaup. Melierax sphenurus, Hartl.

S., W. and N.-E. Africa.

199. Aster badius.

B. M.

Falco badius, Gm. Brown's Illusir. pl. 3. F. Brownii, Shaw. F. Dussumieri, Temm. $\quad P l$. col. 308, 336. Micronisus badius, G. R. Gr. Astur Dussumieri, Cuv. Sparvius badius, Vieill. Nisus Dussumieri, Less. N. manillensis Meyen, Nova Acta, \&c., 1834, Suppl. t. 9. Accipiter dukhunensis, Sykes. A. fringillarius, Jerd. A. scutarius et A. fringillaroïdes, Hodgs. A. badius, G. R. Gr. Astur (Micronisus) badius, Kaup.

India and Malayan Archipelago. 
200. Astur gabar.

B. M.

Falco gabar, Daud. Levaill. Ois. de l'Afr. t. 33. Pl. col. 122, 140. Micronisus gabar, G. R. Gr. Pr. B. M. niger, $P r . B$. Nisus gabar et N. niger, Cuv. Accipiter erythrorhynchus, $S w$. A. gabar, Vig. Astur (Micronisus) gabar, Kaup. Falco Banksi, Temm. Sparvius niger et S. leucorrhous, Vieill. Accipiter niger, Gould, Syn. B. of Austr. iii. pl.f. 1. Melierax gabar, Hartl.

Greece and Africa.

201. Astur monogramuicus.

Falco monogrammicus, Temm. Afr. i. pl. 4 Accipiter. Pl. col. 314. Sw. B. of W. Afr. 1. pl. 4. Accipiter monogrammicus, G. R. Gr. Astur monogrammicus, Cuv. Micronisus monogrammicus, $\boldsymbol{P r} . \boldsymbol{B}$. Astur (Micronisus) monogranmicus, Kaup. Nisus monngram-

W. Africa. micus, Less. Melierax monogrammicus, Hartl.

\section{ACCipiter.}

a. Accipiter, Briss. 1760.

Nisus, Cuv. 1799-1800.

Terax, Leach, 1816.

202. Accipiter nisus.

Falco nisus, $L$. $P l$. enl. 467,41 nisus, Vieill. Accipiter 1 . F. lacteus, Gm. Sparvius of Eur. i. 11. 18. A frould, B. A. subtypicus, H. fringillarius, Ray. A. nisosimilis, Tick. fringillarum, Hodgs. Nisus communis, $C u v$. N. elegans, N. Ierax fringill $\mathrm{N}$. peregrinus, Brehm. N. fringillarius, Kaup. Keys \& Bl. A A 2 edit. i. fig. p. 67.

Great Britain, Europe, Asia minor, Japan and N.-E. Africa.

203. Accipiter ertithronemia.

Accipiter erythronemia, G.R. Gr Fol B. M .

S. America (Chili, Brazil !)

204. Accipiter tachiro.

Falco tachiro, Daud. Levaill. Ois. 420. F. polyzonos, Temm . As. de l'Afr. t. 24. Pl. col.377, Abyss. t. 18, f. 1. Buteom. F. unduliventer, Rüpp. Fauna R. $G r$. Nisus polyzonos tachiro, Cuv. Accipiter tachiro, $G$. renter, $R$ üpp. Astur tachips. N. tachiro, Cuv. N. unduliN.-E. and S. Africa. 
205. Accipiter refiventris.

B. M.

Accipiter rufiventris, A. Smith, Ill. Zool. S. Afr. Birds, pl. 93. Falco exilis, Temm. Pl. col. 496. F. perspicillatus, Rüpp. Fauna Abyss. t. 18, f. 2. Nisus perspicillatus, Kaup. N. exilis, Less. Dædalion perspicillatus, Rüpp.

N.-E. and S. Africa.

206. Accipiter fuscus.

B. M.

Falco fuscus, Gm. F. pennsylvanicus, Wils. Amer. Orn. pl. 46, f. 1, Pl. col.67. F. velox, Wils. Amer. Orn. pl. 45, f. 1. F. dubius et F. obscurus, $G m$. Accipiter fuscus, G.R. $G r$. A. striatus, Vieill. Ois. de l'Amer. Sept. t. 14. A. pennsylvanicus et A. velox, Steph. A. mexicanus, $S w$. A. fringilloïdes, $V i$ gors? Nisus Malfini, Less. N. pennsylvanicus et N. velox, Cuv. N. striatus, D'Orb. \& Lafr. Sparvius lineatus, S. striatus et $\mathbf{S}$, ardosiacus, Vieill.

N. and S. America.

207 ? ACCipiter ferrugineus,

Falco ferrugineus, Erman, Verz. Thier und Pflang. p. 16.

S. America (Brazil).

208. Accipiter pileatus.

B. M.

Falco pileatus, $\operatorname{Pr} . \mathbf{M a x} . \quad \mathrm{Pl}$. col. 205. Nisus pileatus et N. variatus, Cuv. Accipiter pileatus, Steph. Sparvius guttatus et S. major, Vieill.

S. America (Brazil, Chili).

209. Accipiter Cooperi.

Falco Cooperi, Pr. B. Amer. Orn. pl. 10, f. 1. F. Stanleyi, Audub. B. of Amer. pl. 56. Accipiter Cooperi, G. R. Gr. Astur Cooperi, Pr. B.

N. America (New Jersey).

210. Accipiter madagascariensis. Accipiter madagascariensis, Verr.

B. M.

Madagascar.

b. Hieraspiza, Kaup, 1844.

211. Accipiter tinus.

Falco tinus, Lath. F. superciliosus, $L . ?$ Accipiter tinus, $G$. $R$. Gr. Sparvius minutus et S. subniger, Vieill. Nisus (Hieraspiza) tinus, Kaup.

S. America. 
212. Accipiter minullus.

B. M.

Falco minullus, Daud. Levaill. Ois. de l'Afr. t. 54. Accipiter minullus, Steph. Nisus minullus, Cuv. Nisus (Hieraspiza) minullus, Kaup.

S. Africa.

213. Accipiter virgatus.

B. M.

Falco virgatus, Temm. Pl. col. 109. F. minutus, Linn.? F. Brissonianus, Shaw? F. Delafonii, H. Smith, Griff. Anim. Kingd. i. pl. p. 239. Accipiter virgatus, Steph. A. besra, Jerd. Ill. Ind. Orn. pl. 4. A. affinis, Hodgs. A. nisoïdes, Bl.? Nisus virgatus, Cuv. N. minutus, Less. N. gularis, Temm. \&. Schl. Fauna Jap. t. 2. Nisus (Hieraspiza) virgatus, Kaup.

N. India, Java and Japan.

\section{c. Urospiza, Kaup, 1845.}

214. ACCIPITE R CIRRHOCEPHalUs.

B. M.

Sparvius cirrhocephalus, S. tricolor et S. melanops? Vieill. Accipiter cirrhocephalus, G. R. Gr. A. torquatus, Vig. $\oint$ Horsf. Gould, B. of Austr. i. pl. 19. F. nisus, var. et F. melanops, Lath.? Nisus australis, Less. Nisus (Urospiza) torquatus, Kaup. Astur (Micronisus) torquatus, Kaup. Nisus tricolor, Kaup.

Australia.

215. ACcipiter torQuatus.

Astur cruentatus, Gould, B. of Austr. i. pl. 18. Nisus (Urospiza) crueutus, Kaup. Falco torquatus, Temm. Pl.col. 43, 93. Accipiter cruentatıs, G. R. Gr. Nisus torquatus, Kuup.

W. and N.? Australia.

216. ACCIPITE R APPRoximans.

B. $\mathrm{M}$.

Astur approximans et A. fasciatus, Vig. \& Horsf. A. radiatus, Cuv. Gould, B. of Austr. i. pl. 17. Falco radiatus, Temm. $P l$. col. 123. Astur radiatus, Cux. Nisus (Urospiza) radiatus et N. (U.) approximans, Kaup. Accipiter approximans, G. $\boldsymbol{R}$. Gr.

New South Wales and S. and N. Australia. 
217. Accipiter radiatus.

B. M. Falco radiatus, Lath. Gen. Syn. Suppl. pl. 121. Gould, B. of Austr. i. pl. Astur radiatus, Steph. A. testaceus, (Emest) Kaup. Sparvius radiatus, Vieill. Haliätus Calei, Vig. § Horsf. Accipiter radiatus, G. R. Gr.

New South Wales.

218.? Accipiter hiogaster.

Falco hiogaster, Müll. \& Schl. Accipiter virgatus, p. G. $R$.

Gr. A. hiogaster, $\operatorname{Pr}$. 13. Nisus (Urospiza) hiogaster, Kaup, Voy. au Pole Surl, Ois. t. 2, f. 1.

Amboina.

$$
\text { d. Tachyspiza, Kaup, } 1844 .
$$

219. Accipiter soloensis.

B. M.

Falco soloënsis, Horsf. F. cuculö̈des, Temm. Pl. col. 110 , 129. Nisus soloëusis, Less. N. cuculoïdes, Cuv. Accipiter soloënsis, Steph. Nisus (Tachyspiza) soloënsis, Kaup.

N. China and Java.

e. Scelospiza, Kaup, 1847.

220. Accipiter Francesit.

B. M.

Accipiter Francesii, A. Smith. Nisus (Scelospiza) Francesii, Kaup.

Madagascar.

221 ? ACCIPITER CARBONARIUS.

Accipiter carbonarius, Licht.

Africa.

\section{Herpetotheres.}

a. Herpetotheres, Vieill. 1818.

Physeta, Vieill. 1816.

Macagua, Less. 1831.

Cachinna, Flem. 1822.

222. HerpetothFres CaCHINNANS.

B. M.

Falco cachinnans, $L$. Spix, Av. Bras. t. 3, a. Herpetotheres cachinnans, Vieill. Gal.des Ois. t. 19. Cachinna herpetotheres, G. R. Gr. Astur cachinnans, Cuv. Macagua cachinnans, Less. Circaëtus (Herpetotheres) cachinnans, Kaup.

S. America, (Brazil, Guiana) and Mexico. 
b. Micrastur, G. R. Gr. 1841 .

Brachypterus, Less. 1837.

Climacocercus, Cab. 1844.

Camifex, Less.

Climacourus, Pr. B. 1850.

223. Herpetotheres Melanoledcus.

B. M.

Falco brachypterus, Temm. $P l$. col. 141, 116. F. leucomelas, Ill. Sparvius semitorquatus, S. ruficollis et $\mathbf{S}$. melanoleucus, Vieill. Nisus brachypterus, Cuv. Astur brachypterus, Steph. Micrastur brachypterus, G.R. Gr. Circaëtus (Herpetotheres) melanoleucus, et C. (H.) brachypterus, Kaup.

S. America (Brazil, Paraguay, Guiana).

224. Herpetotheres concentricus.

B. M.

Falco concentricus, Ill. Nisus concentricus, Less. Micrastur concentricus, G. R. Gr. Sparvius gilvicollis, Vieill. Circaëtus (Herpetotheres) concentricus, Kaup. Climacocercus concentricus, $C a b$.

S. America (Peru).

225. Herpetotheres xanthothorax,

B. M

Falco xanthothorax et F. leucauchen, Temm. Pl. col. 92, 306. Astur leucauchen, Cuv. Astur xanthothorax, Steph. Nisus xanthotborax, Cuv. N. leucauchen, Less. Micrastur xanthothorax, G. R. Gr. Circaëtus (Herpetotheres) xanthothorax, Kaup.

S. America (Guiana, Brazil).

226. Herpetotheres guerilla.

Micrastur guerilla, Cass.

Mexico.

\section{Geranospiza.}

Geranospiza, Kaup, 1847.

Ischnoscelis, Strickl. 1844.

Geranopus, Kaup, 1850.

227. Geraxospiza gracilis.

B. M.

Falco gracilis et F. hemidactylus, Temm. Pl. col.91, 3. F. Wiedii, brasiliensis, Griff. Anim. Kingd. i. pl.p. 238. Nisus hemidactylus, Cuv. N. gracilis, Less. Sparvius cærulescens, Vieill. Astur hemidactylus et A. gracilis, Steph. Brachypterus hemidactylus et B. gracilis, Less. Ischnoscelis gracilis, Strickl. Geranospiza gracilis et Geranopus gracilis, Kaup.

S. America (Brazi], La Plata) and the Island of Puna. 
228.? Geranospiza nigra.

Ischnocelis niger, Dubus. Geranospiza vigra, G. R. Gr. S. America.

vii. Circinæ.

Circinæ, Pr. B. 1838-49-50. G. R. Gr. 1840-41-45. Bl. 1849.

Buteonina, p. Vigors. 1825.

Falconinæ, p. $\operatorname{Pr}$. B. 1831.

Buteoniuæ, p. Sw. 1837.

Milvinæ, p. et Aquilinæ, p. Kaup, $184 \%$.

Polyborinæ, p. Pr. B. 1849.

\section{Circus.}

a. Circus, Lacep. 1800-1801. .

Pygargus, Koch, 1816.

229. Circus exteinosus.

Falco æruginosus, $L$. $P l$. enl 460,424 . F rufus, $G$ B. M. arundinaceus, Bechst. F. Krameri, Kram. Circus reruginosus, Sav. C. variegatus, Sykes. C. rufus, var, indicus et C. Sykesi, Less. C. rufus, Eytun. Buteo æruginosus, Flem. B. rufus, Jenyns. Accipiter circus, Pall. Pygargus rufus, Koch. Gould, B. of Eur. pl. 32. Yarr. Brit. B.2 edit. i. fig. p. 97. Great Britain ; Europe, Asia and N.-E. Africa.

230. Circus Ravivorus.

B. M.

Falco ranivorus, Daud. Levaill. Ois, de l'Afr. t. 23. Circus ranivorus, Vieill. Pygargus ranivorus, Kaup.

S. Africa.

231. Circos Gouldir.

Circus assimilis, Gould, B. of Austr. pl. 26. C. Gouldii, Pr. B.

Australia (except the Northern) and New Zealand.

232. Circus spiloyotus.

Circus spilonotus, Kaup.

India. 
b. Strigiceps, Pr. B. 1831 .

233. Circus cyaneus.

B. $\mathrm{M}$

Falco cyaneus, et F. pygargus, $L . \quad P l$. enl. 443,480 . F. bohemicus, F. albicans, F. griseus, et F. montanus, Gm. F. cinereus, et F. rubiginosus, St. Poseg. F. strigiceps, Nils. Accipiter variabilis, Pall. Circus cyaneus, Boie. C. gallinarius, Sav. C. pygargus, Cuv. C. Egithus, Leach. Pygargus dispar, Koch. Buteo cyaneus, Jenyns. Strigiceps pygargus, et S. cyaneus, Pr. B. Gould, B. of Eur. pl. 33. Yarr. Brit. B. 2 edit. i. fig. p. 101. Falco hudsonius, L. Wils. Amer. Orn. pl.. 51, 1. 2. Vieill. Ois. de l'Amer. Sept. t. 9. Pr. B. Amer. Orn. pl. 12. F. uliginosus, F. variegatus, F. albidus, et F. Buffonii, Gm. F. europogistus, Bosc. Visill. Ois. de l'Amer. Sept. t. 8. Circus hudsonius, C. uliginosus, et C. variegatus, Vieill. Circus (Strigiceps) cyaneus, $\operatorname{Pr}$. B. C. (S.) uliginosus, Kaup. Buteo (Circus) cyaneus? var.? Americanus, Sw. \& Rich. Fauna Bor.-Amer. ii. pl. 29. Strigiceps hudsonius, $\operatorname{Pr} . B$.

Great Britain; Europe, Siberia, N. Africa and N. America.

234. Circus melayoleucus.

B. M.

Falco melanoleıcus, Gm. Levaill. Ois. de l'Afr. t. 32. F. herbicola, Tick. Circus melanoleucus, Vieill. Circus (Strigiceps) melanoleucus, Kaup. Strigiceps melanoleucus, $\operatorname{Pr}$. $B$. India.

235. Circus cinereus.

B. $\mathrm{MI}$.

Falco histrionicus, Quoy \& Gaim. Voy. de l'Uranie, t. 15, 16. Circus cinereus, Vieill. C. poliopterus, Cab.? Circus (Strigiceps) histrionicus, Kaup. Strigiceps histrionicus, $\operatorname{Pr} . B$.

S. America (Chili) and Falkland Islands.

236? Circus acoli.

B. M.

Falco acoli, Daud. Levaill. Ois. de l'Afr. t. 31. Circus acoli, Vieill. C. cinerens, p. Kaup. Circus (Strigiceps) acoli, Kaup. Strigiceps histrionicus, p. $\operatorname{Pr}, B$.

S. Africa?

237. Circes frenatus.

Falco frenatus, $I l l$. Circus campestris. Vieill. C. cinereus, p. G. R. Gr.

S. America. 
238. Circus ater.

B. M.

Circus ater, Vicill. C. Lalandi, A. Smith, Ill. S. Afr. Zool. Birds, pl. 58. Falco maurus, Temm. Pl. col. 461. Circus (Strigiceps) maurus, Kaup. Strigiceps ater, Pr. B. C. cineraceus, var.?

S. and N.-E. Africa.

c. Glaucopteryx, Kaup, 1844.

239. Circus cineraceus.

B. M.

Falco cineraceus, et F. cinerarius, Mont. Gould, B. of Eur. pl. 35. Yarr. Brit. B.2 edit. i. fig. p. 107. Circus cineraceus, Cuv. C. Montagui, Vieill. C. cineraceus, s. pallidus, et C. nipalensis, Hodgs. C. cinerarius, Leach. C. cinerascens, Steph. Buteo cineraceus, Flem. Circus (Glaucopteryx) cineraceus, Kaup. Strigiceps cinerasceus, Pr. $B$.

Great Britain; Europe, and India.

240. Circus Swainsoni.

B. M.

Circus Swainsoni, A. Smith, Ill. S. Afr. Zool. pl. 43, 44. C. albescens, Less. C. pallidus, Sykes. C. dalmaticus, Rüpp. Mus. Senk. 1834, t. 11. C. Feldeggii, Bruch. Falco æquipar, Cuv. MSS. Circus (Glaucopteryx) Swainsoni, Kaup. Strigiceps Swainsoni, $\operatorname{Pr} . B . \quad P l$. enl. 459 .

Dilmatia, Greece, India and S. Africa.

\section{d. Spizacircus, Kaup. 1845.}

241. Circus macropterus.

B. M. Circus macropterus, et C. albicollis, Vieill. C. superciliosus, Less. Tr. d'Orn. t. 3, f. 1. C. megaspilus, Gould. C. palustris, Cuv. Falco palustris, $\operatorname{Pr}$. Max. Circus (Spizacircus) maciopterus, Kaup. Pl.col. 22.

S. America (Brazil, Cayenne). 
e. Spilocircus, Kaup, 1847.

242. Circts Jardinit.

Circus Jardinii, Gould, B. of Austr. i. pl. 27. C. assimilis, Jard. \& Selby, Illustr. of Orn. pl. 51. Circus (Spilocircus) Jardinii, Kaup. Strigiceps Jardinii, $\operatorname{Pr} . B$.

New South Wales and S. Australia.

Examine, Circus cærulescens, Vieill.

S. America.

Circus leucophrys, Vieill.

India.

Falco javanica, Gm. Circus javanicus, Vieill. Java.

Circus approximans, Peale, U. S. Expl. Exped. viii. p. 64, pl. 18.

Feejee Islands.

\section{Polyboroides.}

Polyboroïdes, A. Smith, 1830.

Gymnogenys, Less. 1830.

243. Polyboroides radiatus.

B. $\mathrm{M}$.

Vultur radiatus, Scop. Sonn. Voy. Ind. t. 103. Falco madagascariensis, Daud. F. gymnogenys, Temm. Pl. col. 307. Sparvius madagascariensis, Vieill. Polyboroïdes, trpus, $A$. Smith. Ill. S. Afr. Zool. pl. 81, 82. P. radiatus, G. R. Gr. Gymnogenys madagascariensis, Less. Nisus gymnogenys, Cuv. Circaëtus (Gymnogenys) radiatus, Kaup.

S.-W. and N.-E. Africa and Madagascar. 


\section{SERPENTARIDA.}

Serpentaridæ, Selys, 1842.

Gypogeranidæ, Steph. 1825. Pr. B. 1831-49-50; Kaup, 1847. Gypogeranides, Sundev. 1835.

Falconidæ, p. G. R. $G r .1840-41$.

\section{i. Serpentarine.}

Gypogeraninæ, Pr. B. 1849-50.

Circinæ, p. G. R. Gr. 1840-41.

\section{Serpentarius.}

Serpentarius, Cuv. 1797-1798.

Sagittarius, Vosm. 1767.

Secretarius, Dum. 1806.

Gypogeranius, Illig. 1811.

Ophiotheres, Vieill. 1816.

244. Serpentaridus secretarius.

B. M. Otis secretarius, Scop. Sonn. Voy. Ind. t. 50. Falco serpentarius, $\mathrm{Gm}$. Pl. enl. 721. Serpentarius reptilivorus, Daud. S. africanus, Shaw. S. cristatus, Less. Levaill. Ois. de l'Afr. t. 25. Ophiotheres cristatus, Vieill. Gypogeranus africanus, $A$. Smith. G. capensis, G. gambensis, et G. philippensis, Ogilby. S.-W. and N.-E. Africa. 


\section{Suborder 1I. ACCIPITRES NOCTURNI.}

Accipitres nocturni, Vieill. 1816; Cuv. 1817.

Nycteriens, Cuv. 1799-1800.

Ėgolii, Vieill. 1816.

Notturni, Ranz. 1823.

\section{STRIGID庄.}

Strigidæ, Leach; Vig. 1825 ; Sw. 1831-37; Pr. B. 1838-49 -50; G.R. Gr. 1840-41-45; Bl. $1849 ;$ Kaup. 1849.

Strixideæ, D'Orb. \&. Lafr. 1837.

Strigides, Sundev. 1835.

Falconidæ, p. Pr. B. 1831.

\section{i. Surnine.}

Surninæ, Pr. B. 1838-49-50; G. R. Gr. 1840-41-45; Cass. 1849 ; Kaup, 1849.

Noctuina, Vig. 1825.

Buboninæ, p. et Atheninæ, $B$ l. 1849.

Atheninæ, Nycteininæ et Striginæ, p. Cass. 1849.

\section{Survia.}

Surnia, Dum. 1806.

Syrnia, Steph. 1826.

245. Surnia ulula.

B. M.

Strix ulula et S. funerea, $L$. Pl. enl. 463. S. doliata, Pall. s. hudsonia, Gm. S. nisoria, Meyer. S. arctica, Sparr. Surnia nisoria, Cuv. Surnia funerea et S. nisoria, Brehm. S. ulula, $\operatorname{Pr} . B$. S. borealis, Less. Syrnium nisoria, Kaup. Gould, B. of Eur. pl. 45. Wils. Amer. Orn. pl. 50, f. 6. Audub. B. of Amex. pl. 378. Yarr. Brit. B. 2 edit. i. fig. p. 146.

Great Britain! N. and W. Europe, Siberia and N. America.

246. Surnia chovcou.

Strix choucou, Lath. S. africana, Shaw. Levaill. Ois. de l'Afr. t. 38. Surnia choucou, Less. S. africana, Steph.

S. Africa.? 
247. SuRnia NisUElla.

Strix nisuella, Lath. Levaill. Ois. de l'Afr. t. 39. Surnia? nisuella, Steph.

S. Africa?

\section{Nyctea.}

Nyctea, Steph. 1825.

Nyctia, Sw. 1837.

248. Nyctea NiveA.

B. M.

Strix nyctea, L. Pl. enl. 458. Gould, B. of Eur. pl. 43. S. nivea, Daud. Levaill. Ois. de l'Afr. t. 45. S. candida, Lath. S erminea, Shaw. Nyctea nivea, $P_{r}$. B. N. erminea, Steph. Nyctia candida, $S w$. Surnia nyctea, Selby. Syrnium nyctea, Kaup. Noctua nyctea, Cuv. N. nivea, Less. Surnia (Nyctea) Kaup. Wils. Amer. Orn. pl. 23, f. 1. Audub. B. of Amer. pl. 121. Vieill. Ois, de l'Amer. Sept. t. 18. Yarr. Brit. $B .2$ edit. i. fig. p. 141.

Great Britain; N. Europe and N. America.

\section{Athene.}

a. Athene, Boie. 1822 .

Noctua, Sav. 1809.

Carine, Kaup, 1829.

249. Athene Noctua.

B. M.

Strix noctua, Retz. Pl. enl. 439. S. passerina, Lath. S. nudipes aut psylodactyla, $N i l s$. S. meridionalis, $R$ isso. S. numida, Levaill. Athene noctua, G.R. Gr. A. psilodactyla, Brehm. A. passerina, Boie. A. gymnopus, Hodgs. A. bactrianus, $B l$. Noctua passerina, Cuv. N.glaux, Sav. N. veterum, Licht. N. meridionalis, Risso. Carine passerina, Kaup. Gould, B. of Eur. pl. 48. Yarr. Brit. B. 2 edit. i. fig. p. 149.

Great Britain; Europe, W. Asia and N. Africa.

250. ATHENE BRAMA.

B. M.

Strix branı, Temm. Pl. col.68. S. indica, Frankl. S. persica, Vieill.? Athene brama, G. R. Gr. A. indica, $B l$. Noctua brama, Cuv. N. tarayensis, Hodgs. N. indica, Jerd. India. 
251. Athene superciliaris.

Strix superciliaris, Vieill. S. Sunneratii, Temm. Pl. col. 21. Athene Sonneratii et A. superciliaris, G. R. Gr. Noctua Sonneratii, Cuv.

India (Pondicherry).

252. Athene sylivatica.

Strix sylvatica, Mïll. Athene sylvatica, $P r . B$.

Sumatra.

253. Athene leucolaima.

- ? Homb. \& Jacq. Voy. au Pole Sud. Ois. t. 4, f. 2, 3. Athene, sp. 4I, G.R. $G r$. Athene leucolaima, Pr. $B$.

Malayan Archipelago.

b. Tænioglaux, Kaup, 1849.

254. Athene cuculoides.

B. M.

Noctua cuculoïdes, Vigors, Gould, Cent. of B. pl. 4. Athene cuculoïdes, $B l$. Noctua auribarbis, Hodgs. Athene (Tænioglaux) cuculoïdes, Kaup.

India (Nepal).

255. Athene castanoptera.

B. MI.

Strix castanoptera, Horsf. S. spadicea, Reinw. Pl. col. 98. Athene castanoptera, $B l$. A. malabarica? et A. castanotus, $B l$.? Noctua castanoptera, Cuv. Athene (Tænioglaux) castanoptera, Kaup.

India (Java, Banda, Sumatra).

256. ATHene erythroptera. Strix erythroptera, Gould. S. radiata, Tick. Athene erythroptera, A. undulata et A. radiata, $B l$. Noctua perlineata, Hodgs. N. cuculoïdes,Jerd. Athene (Tænioglaux) erythroptera, Kaup. India.

257. Athene ocellata.

f ? Homb. \& Jacq. Voy. au Pole Sud. Ois.t. 3, f. 2. Athene, sp. 39, G. R. Gr. Athene ocellata, $\operatorname{Pr} . \boldsymbol{B}$. Malayan Archipelago. 
258. Athene variegata.

Strix variegata, Quoy et Gaim. Voy. de l'Astrol. t. 1, f. 2.

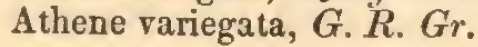

New Ireland.

259. Athene punctulata.

Strix punctulata, Quoy \& Gaim. Voy, de l'Astrol. Ois. t. 1, f. 1. Athene punctulata, $G, R$. $G r$.

Celebes.

260. Athene squamipila.

Athene squamipila, $\operatorname{Pr}$. B. Consp. p. 41.

Ceram.

261. Athene guteruhi. Timor.

Strix (Athene) guterubi, Müll. Verh. Nederl. Ethnogr. p. 279.

\section{Athene JacQuinoti.}

? Homb. \& Jacq. Voy. au Pole Sud. Ois. t. 3.

f. 1. Athene, sp. 38, G. R. Gr. Athene Jacquinoti, Pr. B. Malayan Archipelago.

263. Athene capensis.

Noctula B. M

Noctua capensis, A. Smith, Ill. S. Afr. Zool. pl.53. Athene capensis, A. Smith. Athene (Tænioglaux) capensis, Kaup.

S. Africa.

c. Nyctipetes, $S u$. 1837.

Microglaux, Kaup, 1849.

264. Athene perlata.

B. M.

Strix perlata, Vieill. Levaill. Ois. de l'Afr.t. 284. S. occipitalis, Temm. $P l$. col. 34. Athene perlata, G. R. Gr. Nyctipetes perlatus, Sw. Noctua occipitalis, Cuv. Athene (Microglaux) perlata, Kaup.

S. and W. Africa.

265. Athene LeUCOPSis.

Athene leucopsis, Hartl.

W. Africa (Island of St. Thomas). 
266. Athene licua.

Strix licua, Licht. Verz. Berl. 1842, p. 12. Athene licua, $P r . B$.

S. Africa.

d. Pholeoptynx, Kaup, 1849.

267. Athene cunicularia.

B. M.

Strix cunicularia, Mol. S. grallaria, Temm. Pl. col. 146. Athene cunicularia, $\operatorname{Pr}$. B. Otus cunicularius et Noctua urucurea, Cuv. N. cunicularia, D'Orb. \& Lafr. N. grallaria, Less. Nyctipetes cunicularia, Sw. Glaucidium cunicularium, Less. Athene (Pholeoptynx) cunicularia, Kaup.

S. America (Chili, Maldanado).

268? Athene patagonica.

Athene patagonica, Peale, U.S. Expl. Exped. viii. p. 78, t. 22, f. 2 .

S. America (Patagonia).

269? Athene hypugæa.

Strix cunicularia, Say. S. hypugæa, Pr. B. Amer. Orn. pl. 7, f. 2. S. californica, Audub. B. of Amer. pl. 412.? Surnia cunicularia, Audub. Athene socialis, Gamb. A. hypogæa, $\operatorname{Pr} . B$.

N.-W. America.

270. Athene dominicensis.

Strix dominicensis, Gm. S. cunicularia et S. suinda,? Vieill. Noctua dominicensis, Cab. Athene dominicensis, G. R. Gr.

W. Indies.

\section{e. Glaucidium, Boie, 1826. Scotophilus, p. Sw. 1837.}

271. Athene pumila.

B. $M$.

Strix pumila, $I l l . \quad P l$. col. 30 . S. minutissima, Pr. Max. S. ferox, Vieill. Noctua pumila, Cuv. N. ferox, D'Orb. \& Lafr. Athene pumila, G. R. Gr. A. minutissima, $\operatorname{Pr}$. B. Glaucidium pumilum, Less.

S. America (Brazil, Paraguay, Patagonia, Bolivia). 
272. Athene ferruginea.

B. M.

Strix ferruginea, Pr. Max. Pl. col. 199. S. pumila, Licht. S. phalænoides, Daud.? Vieill. Ois. de l'Amer. Sept. t. 15.? Noctua ferruginea, Cuv. Athene ferruginea, G.R.Gr $\operatorname{Pr}$. B. Glaucidium ferrugineum, Less.

S. America (Brazil) and W. Indies.?

273. Athene Nana.

B. M.

Strix nana, Vigors. Athene nana, G. R. Gr. \& Mitch. Gen. of B. pl. 12. Glaucidium nanum, Boie.

S. America (Str. of Magellan).

274. Athene rufipes.

Strix rufipes, King. Athene rnfipes, G. R. Gr.

S. America (Str. of Magellan).

275. Athene infuscata.

B. MI.

Strix infuscata et S. passerinoïdes, Temm. Pl. col.344. Glaucidium gnoma, Wagl. G. passerinoides, Less. Surnia passerinoïdes, Audub.? Noctua passerinoïles, Less. Athene passerinoïdes et A. gnoma, G. R. Gr. A. infuscata, $P r . B$.

S. America (Brazil).

276. Athene Fusca.

Strix fusca, Vieill. S. Maugei, Temm. Pl, col. 46. Noctua Maugei, Cuv. Athene Maugei et A. fusca, G. R. Gr.

W. Indies (Antilles).

f. Taenioptynx, Kaup, 1849.

277. Athene Brodei.

B. M.

Athene Brodei, $B l$. Noctua Brodei, Burt. N. Iubiger, Hodgs. Athene (Taenioptynx) Brodei, Kaup.

India (Nepal).

g. Ninox, Hodgs. 1837.

278. Athene scutellata.

B. M.

Strix scutulata, Raff. S. hirsuta, Temm. Pl. col. 289. S. lugubris, Tick. S. hirsuta, borneensis et S. hirsuta, japonica, Temm. \&. Schl. Fauna Jap. t. 9, B. Athene scutellata, $G$. R. Gr. A. malayensis, Eyton. A. hirsuta, A. borneensis et A. japonica, $\operatorname{Pr} . \ddot{B}$. Noctua hirsuta, $C u v$. Ninox nipalensis et N. Jeridius, Hodgs. N. scutulatus et N. lugubris, $B l$.

India, (Nepal) Malacca, Ceylon, Cochinchina and Japan. 


\section{h. Sceloglaux, Kaup, 1849.}

279. Athene albifacies.

Athene albifacies, $G . R, G$. $V$. 1. Noctua venation. Ber Birds, 1. Noctua venatica, Peale, U. S. Expl. Exped. viii. p. 75, pl. 22, f. 1. Ninox (Sceloglaux) albifacies, Kaup.

New Zealand.

\section{i. Hieracoglaux, Kaup, 1849.}

280. Athene connivens.

Falco connivens, Lath. Bnteo conoirens, connivens, Bnteo connirens, Vieill. Butaetes connivens, Less. Athene connivens et A. frontata, $G, R . G r$. A.? fortis, Gould, B. of Austr. i. pl. 34. Noctua frontata, Less. Ninox (Hieracoglaux) connivens, Kaup.

New South Wales; S. and W. Australia.

281. Athene strenua.

Athene? strenua, Gould, B. of Austr. racoglaux) strenuus, Kaup.

New South Wales.

282. Athene RUFA.

Athene rufa, Gould, B. of Austr. pl. 36.

N. Australia.

j. Spiloglaux, Kaup, 1849.

283. ATHENE NOVAE ZEALANDIFE.

Strix novæ zealandix, $G m$.

B. $\mathrm{M}$. zealandix, G.R. Gr. Surnia novæ zealandiæ, Quoy \&-Gaim. Voy. de l'alandiæ, Less. Noctua (Spiloglaux) novæ seelandiæ, Kaup.

New Zealand.

284. Athene maculata.

B. M.
Noctua maculata, Vig. \&. Horsf. Athene maculata, Gould, $B$.

of Austr. i. pl. 33. Ninox (Spiloglaux) maculatus, Kaup.

New South Wales, S. Australia and Van Diemen's Land. 
285. Athene marmorata.

Athene marmorata, Gould. Ninox (Spiloglaux) marmoratus, Kaup.

S. Australia.

286. Athene воовоок.

B. M.

Strix boobook, Lath. Athene boobook, Gould, B. of Austr. i. pl. 32. Noctua boobook, Vig. \& Horsf. Ninox boobook, Bl. Ninox (Spiloglaux) boobook, Kaup.

Australia.

k. Microptynx, Kaup, 1849.

287. ATHENE PASSERINA.

B. M.

Strix passerina, L. Gould, B. of Eur. pl. 50. S. acarlia, Temm. S. pusilla, Daud. Levaill. Ois. de l'Afr. t. 46. S. pygmæa, Bechst. Athene acadiaca, Boie. A. passerina, G. R. Gr. Glancidium passerinum, Boie. Surnia (Microptynx) passerina, Kaup.

N. Europe ; S. and N.-E. Africa.

288. Athene elata.

Strix elata, Natt. Glaucidium et Athene elata, Pr. $B$.

Mexico.

\section{Nyctale.}

Nyctale, Brehm, 1828.

Scotophilus, Sw. 1837.

Egolius, Kaup, 1829.

289. Nyctale funerea.

B. M.

Strix funerea, $L . \quad E d w$. B. pl. 228. S. passerina, Pall. S. Tengmalmi, Gm. Gould, B. of Eur. i. pl. 49. Yarr. Brit. B. 2 edit. i. fig. p. 153 . Nyctale funerea, $\operatorname{Pr}$. $B$. N. pinetorum, N. abietum et N. planiceps, Brehm. N. Tengmalmi et N. Richardsoni, Pr. B. Scotophilus Tengmalmi, Sw. Noctua Tengmalmi, Cuv. Aggolius TengmaImi, Kaup. Ulula Tengmalmi, Audub. B. of Amer. pl. 380.

Great Britain; Siberia, N. Europe and N. America. 
290. Nyctale acadica. B. M. Strix acadica, Gm. S. acadiensis, Lath. S. passerina, Wils. Amer. Orn. pl. 34, f. 1. S. albifrons, Turt. Shaw, Nut. Misc. pl. 17l. S. Wilsonii, Boie. S. frontalis, Licht. Strix (Noctua) acadica, Rich. \&.Sw. Nyctale acadica, Pr. B. N. Harrisii, Cass.? Noctua acadica, Less. Scotophilus acadicus, Sw. Ulula acadica, $A u d u b$.

N. America .

291. Nyctale siju.

Noctua siju, D'Orb. Voy. l'ile de Cuba, Ois. t. 3. Athene siju, G. R. Gr. Nyctale siju, $\operatorname{Pr} . B$.

Cuba.

\section{ii. Bubonine.}

Buboninæ, Pr. B. $1838-49 ;$ G. R. Gr. 1840-41-45; Bl. 1849 ; Cass, 1849.

Bubonina, Vig. 1825.

Striginæ, p. Kaup, 1849.

Surninæ, p. Pr. B. 1850.

Aëtoglaucinæ, Hodgs. 1837.

\section{Buвo.}

a. Bubo, Sibb. 1684.

Feliceps, Barr.

Asio, Briss. 1760.

Heliaptex, Sw. 1837.

Bubotus, Rafinq. 1815.

Ascalaphia, J. Geoffr. 1837.

292. Bubo мaximos.

B. M.

Strix bubo et S. scandiaca, L. Pl. enl. 435. Gould, B. of Eur. i. pl. 37. Bubo maximus, (Sibb.) Flem. B. atheniensis, B. albus et B. nudipes? Daud. B. europeus, Less. B. germanicus et B. septentrionalis, Brehm. B. microcephalus, Leach. B. sibiricus et B. turcomana? nec leucomanna, Eversm. B. cinereus, G. R. Gr. \& Mitch. Gen. of B.pl. 13. Asio bubo, Sw. Yarr. Brit. B. 2 edit. i. fig. p. 114.

Great Britain; Europe, N. Africa and Siberia. 
293. Bubo Capensis.

B. M.

Bubo capensis, Daud. Levaill. Ois. de l'Afr. t. 40. Ill.S. Afr. Zool. pl. 70. B. Dilloni, O. des Murs. Strix africanus, Steph.

S. and N.-E. Africa.

294. Bubo Bengalensis.

B. M.

Otus bengalensis, Frankl. Gould, Cent. of B. pl. 3. Bubo bengalensis, G. R. Gr. B.? cavearius et Urrua cavearia, Hodgs.

India.

295. Bubo ascalaphus.

B. M.

Bubo ascalaphus, Sav. Descr. de l Egypte, Ois.t. 3, f.2. Strix (Bubo) ascalaphus, $A u d$. Otus ascalaphus, Cuv. Ascalaphia Savigni, G. R. Gr. Pl. col.57. Gould, B. of Eur. pl. 38.

S. Europe, N.-E. Africa, Persia, Sardinia? and Sicily.?

296. Bubo virginianus.

B. M.

Strix virgiuiana, Gm. Edw. B. pl. 60. Wils. Amer. Orn. pl. 50, f. 1. Strix (Bubo) virginiana, Rich. \& Sw. Asio virginiana, Ŝw. Bubo pinicoia, Vieiil. Vis. de l̈Amer. S̈ept. t. 19. B. virginianus, Less. B. ludovicianus, Daud. Strix (Bubo) arcticus, Sw. \& Rich. Fauna Bor-Amer.pl. 30. Heliaptex arcticus, $S w$. Audub. B. of Amer. pl. 61. Strix magellanica, Gm. Pl.enl. 385. S. nacurutu, Vieill. Bubo magellanicus, Cuv. Otus nacurutu, Less. Strix crassirostris, Vieill. S. macrorhynchus, Temm. Pl. col. 64. Otus macrorhynchus, Cuv. O. crassirostris, G. R. Gr.

N. and S. America.

297. Buno macolosus.

B. M.

Strix maculosa, Vieill. S. africana, Temm. Pl.col. 50. Otus maculosus, Less. O.'africanus, Cuv. Bubo africanus, Boie. B. rvaculosus, Cass.

S. and W. Africa.

b. Urrua, Hodgs. 1837.

Mesomorpla, Hodgs. 1841.

298. Bubo coromaxper.

B. M.

Strix coromandra, Lath. Ill. Ind. Zool. pl. 20. Bubo coromander. G. R. Gr. B. umbratus, $B l$. Urrua coromandra, Hodgs. U. umbrata, Bl. Bubo (Urrua) coromander, Kaup. India. 
299. Bubo Lacteus.

Strix lactea, Temm. Pl.col.4. Bubo lacteus, Cuv. Pr. B. B. sultaneus, Less. B. Verreauxi, $\operatorname{Pr} . B$. Scops lactea, Less. Otus lacteus, Less. Bubo cineraseens, Guer. Ferr. \& Gal. Voy. Abyss. Ois. t. 2. Pr. B. Bubo (Urrua) lacteus, Kaup.

S., W. and N.-E. Africa.

c. Huhua, Hodgs. 1837.

Etoglaux, Hodgs. 1841.

Urrua, p. Kaup, 1849.

300. Bubo orientalis.

B. M.

Strix orientalis, Horsf. S. sumatrana, Raff. S. strepitans, Temm. Pl. col. 174, 229. Bubo orientalis, G. R. Gr. B. strepitans, Cuv. Syrnium strepitans et Lophostrix sumatrana, Less. Bubo (Huhua) nepalensis et Hulua nepalensis, Hodgs. H. pectoralis, Jerd. Madr. Journ. 1839, pl. 1. Bubo (Urrua) orientalis, Kaup.

India (Nepal), Java, Sumatra .

d. Pseudoptynx, Kaup, 1849.

301. Brbo philippensis.

B. M Syrnium philippense, G. R. Gr. Bubo (Pseudoptynx) philippensis, Kaup.

Philippine Islands.

e. Ketupa, Less. 1831.

Cultrunguis, Hodgs. 1836.

302. Bubo cejlonensis.

B. $M$. Strix ceylonensis, Gm. Brown, Illustr. pl. 4. S. Leschenaultii, Temm. Pl. col. 20. S. Hardwickii, Gr. Ill. Ind. Zool. pl. 31. S. dumeticola, Tick. Ketupa ceylonensis, G. R. Gr. K. Leschenaultii, Less. Cultrunguis nigripes, Hodgs. C. Leschenaultii, Jerd. Bubo (Ketupa) ceylonensis, Kaup.

India, (Nepal, Behar, Madras).

303. Bubo javanensis.

B. M.

Ketupa javanensis, Less. Strix ceylonensis, Temm. Pl. col. 74. S. Ketupa, Horsf. Scops ketupa, Cur. Bubo (Ketupa) ketupa, Kaup.

Java, Sumatra and Ceylon. 
304. Bubo flavipes. B. M. Cultrunguis flavipes, Hodgs. Ketupa flavipes, G.R. Gr. Bubo (Ketupa) flavipes, Kaup.

India (Nepal).

f. Scotopelia, Pr. B. 1850.

305. Bubo Peli.

B. M.

Strix Peli, Temm. Pr. B. Consp. p. 44.

Ashantee.

\section{Ephialtes.}

a. Ephialtes, Keys. \& Bl. 1840.

Scops, Sav. 1809.

306. Ephialtes scops.

B. M. Strix scops, L. Pl. enl. 436. S. zorca, Cetti. S. carniolica, Gm. S. gui, Scop. S. pulchella, Pall. Scops zorca, $S w$. S. Aldrovandi, Flem. S. ephialtes, Sav. S. europæus, Less. S. carniolica, Brehm. S. vulgaris, Rüpp. Bubo scops, Boie. Ephialtes scops, Keys. \& Bl. Otus scops, Schl. Gould, B. of Eur. pl. 41. Yarr. Brit. B. 2 edit. i. fig. p. 120. Scops senegalensis, Sw. S. capensis, A. Smith. S. rutilus, Pucher. Archiv. de Mus. iv. t. 22.? Otus (Scops) africanus, Temm. \& $\boldsymbol{S c h l}$. Scops pennata et S. sunia, Hodgs. S. malayanus, $\boldsymbol{A}$. Hay. Otus (Scops) japonicus, Temm. \&. Schl. Fauna Jap. t. 9. Ephialtes spilocephalus, $B l$. E. sunia, $G . R$. $G r$.

Great Britain; Europe, Africa, Madagascar, India and Japan.

\section{b. Psilorhina, Kaup, 1849.}

307. Ephialtes manadensis.

Strix manadensis, Quoy et Gaim. Voy. de $l$ Astrol. t. 2, f. 2. Ephialtes manadensis, G. $R$. Gr. Scops manadensis, Less. Otus manadensis, Schl. Scops (Psilorhina) manadensis, Kaup). Celebes. 
c. Megascops, Kaup, 1849.

308. Ephialtes Lempuit.

B. MI.

Strix indica, Gm.? S. lempjii et S. rufescens, Horsf. S. noctula, Reinw. Pl. col.99. Scops lettia, Hodgs. S. malabaricus, S. griseus et S. lettoïdes, Jerd. S. javanica, Less.? S. lempjii, S. inantis et S. sagittatus, $P r$. $B$. Otus noctula et Otus mantis, Temm. \& Schl. Ephialtes lempjii, G.R.Gr. E. sagittatus, Cass. Scops (Megascops) indica, Kaup.

India, Java, Sumatra, Banda, Borneo and Ceylon.

309. Ephiates magica.

Otus magicus, Temm. \&-Schl. Strix magica, Mïll. Ephialtes magicus, G. R. Gr. Scops magicus, Pr. B.

Amboina, Celebes.

310. Ephialtes semitorques

B. M. Otus semitorques, Temm. \& Schl. Fauna Jap. t. 8. Ephialtes semitorques, G. R. Gr. Scops semitorques, $P r . B$.

Japan.

311. Ephiates nove Zealande.

Scops novæ zealandiæ, $\operatorname{Pr}$. B. Consp. p. 47.

New Zealand. (?)

312. Ephialtes asio.

B. M.

Strix asio, L. Wils. Amer. Orn. pl. 42, f. 1. S. næria, Gm. Wils. Amer. Orn. pl. 19, f. 1. Otus nævius, Cuv. O.asio, Schl. Scops asio, Less. Bubo asio, Audub. Ephialtes asio, G. R. Gr. Scops (Megascops) asio, Kaup. Pl. col. 80. Audub. B. of Amer. pl. 97. Vieill. Ois. de l'Amer. Sept. t. 21.

N. America.

313. Ephiates atricapilla.

B. M.

Strix atricapilla, Natt. $P l$. col. 145. Scops atricapilla, Cuv. Ephialtes atricapilla, G.R.Gr. Scops (Megascops) atricapilla, Kaup.

S. America (Brazil). 
314. Ephialtes choliba. Strix brasiliana, Gm.? S. choliba, Vieill. S. decussata, Licht. S. crucigera et S. undulata, Spix, Av. Bras. t. 9, 10 . Ephialtes choliba, G. R. Gr. Otus brasiliensis, Temm. \&. Schl. Scops brasiliensis, $\operatorname{Pr}$. B. S. choliba, D'Orb. \& Lafr. S. brasiliana, S. decussata et S. crucigera, Less. S. portoriensis, Less. O. des Murs, Iconogr. Omith. t. 26. Scops (Megascops) brasiliana, Kaup.

S. America (Brazil, Caraccas, Bolivia and Argentina).

\section{Ephialtes lophotes.}

Scops lophotes, Less. Ephialtes? lophotes, G. R. Gr. E. Watsoni, Cass.?

S. America.

316. Ephialtes albopunctata.

B. M.

Ephialtes albopunctata, G. R. Gr. Scops (Megascops) albopunctata, Kaup.

S. America.

317. Ephialtes trichopsis.

Scops trichopsis, Wagl. Ephialtes trichopsis, G. R. Gr.

Mexico.

$$
\text { d. Acnemis, Kaup, } 1849 .
$$

318. Ephiltes gymnopodus.

B. M.

Scops gymnopodus, et Ephialtes gymnopodus, G. R. Gr. Scops (Acnemis) gymnopodus, Kaup.

India.

319. Ephialtes nudipes.

Bubo nudipes, Vieill. Ois. de l'Amer. Sept.t. 22. Ephialtes? nudipes, G. R. Gr. Scops (Acnemis) uudipes, Kaup.

W. Indies.

320? Eyhialtes Daudin.

Strix nudipes, Daud. Vieill. Ois. de l'Amer. Sept.t. 16. Noctua nudipes, Cuv. Athene? nudipes, G. R. Gr.

W. Indies (Antilles) 
e. Ptilopsis, Kaup, 1849.

321. Ephialtes leccotis.

B. M.

Strix leucotis, Temm. Pl. col. 16. Otus leucotis, Cuv. Scops leucotis, $S w$. Ephialtes leucotis, G. R. Gr. Pr. B. Scops (Ptilopsis) leucotis, Kaup.

N.-E. and W. Africa.

f. Lophostrix, Less. 1837.

322. Ephialtes cristata.

B. M.

Strix cristata, Daud. S. griseata, Lath. Levaill. Ois, de l'Afr.

t. 48. Jard, Contr. Ornith. pl. var. Bubo griseatus, Cuv. Syrnium griseatum, Less. Ephialtes cristata, G.R. Gr. Lophostrix griseata, Less. L. cristata, Pr. B. Bubo (Lophostrix) cristatus, Kaup.

S. America (Cayenne).

iii. Syrninate.

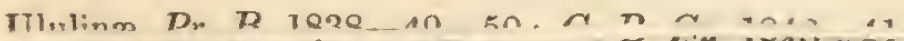

Syrniinæ, G. R. Gr. 1845 ; Bl. 1849.

Syrniana, Vig. 1825.

Striginæ, p. Vig. 1825 ; Cass. 1849 ; Kaû́p, 1849.

Buboninæ, p. $B$ l. 1850.

\section{Syrnium.}

a. Syrnium, Sav. 1809.

Úlula, Cuv. 1817.

Scotiaptex, $S w .1837$.

Aluco, Kaup. 1829.

323. Syrnium aluco.

B. M.

Strix aluco et S.stridula, $L$. $P l$. enl.441, 437. S. soloniensis, Gm. S. sylvatica, Shaw. S. alba, S. sylvestris, S. noctua et S. rufa, Scop. Syrnium aluco, Cuv. S. ululans, Sav. S. aluco et S. stridulum, Brehm. Ulula aluco, Macgill. Gould, B. of Eur. pl. 47. Yarr. Brit. B. 2 edit. i. fig. p. 138.

Great Britain; Europe, N. Africa and Japan.

324. Syrnium nivicolum.

B. M.

Syrnium nivicolum, Hodgs. Urrua nivicola et Mesomorpha nivicola, Hodgs.

India (Nepal). 
325. Syrnium cinereum.

B. M.

Strix cinerea, Gm. S. lapponica, Retz. S. barbata, Pall. S. acclamator, Bartr. S. fuliginosa, Shaw. S. microphtalmus, Tyzensk. Syrnium cinereum, Pr. B. S. microphtalmum, $G$. R. Gr. Scoliaptex cinerea, Sw. Ulula lapponica, Cuv. U. cinerea, $\boldsymbol{P r}$. B. Surnia lapponica, Less. Sparr. Mus. Carls. t. 5. Gould, B. of Eur. pl. 42. Fauna Bor.-Amer. ii. pl. 31 . Audub. B. of Amer. pl. 351.

N. Europe, Siberia and N. America.

326. Syrnium nebulosum.

B. M.

Strix nebulosa, Gm. Gould, B. of Eur. pl. 46. Syrnium nebulosum, Boie. Ulula nebulosa, Cuv. Wils. Amer. Orn. pl. 33, f. 2. Vieill. Ois. de l'Amer. Sept t. 17. Audub. B. of Amer. pl. 46. Shaw, Nat. Miscell. pl. 25.

N. America.

b. Ptynx, Bl. 1840.

327. Syraidu uRALENSIS,

B. M.

Strix uralensis, Pall. S. literata, Retz. Pl. col. 27. S. macroura, Natt. S. macrocephala, Meisn. Syrnium uraleusis et S. macrocephalon, Brehm. S. uralense, G.R. Gr. Scotiaptex uralensis, Sw. Ptynx uralensis, Bl. Noctua (Surnia) uralensis, Cuv. Surnia uralensis, Pall. Ulula litturata, Cuv. Lepechin. Voy. ii. t. 8.

N. and E.-N. Europe, Ural Mountains, Siberia and N. America.

\section{8 ? Syrnium FUSCESCENS.}

Strix rufescens aut fuscescens, Temm. \& Schl. Fauna Jap. t. 10. Strix uralensis, Temm. Syrnium fuscescens, $G, R$. $G r$. Ptynx fuscescens, $\operatorname{Pr}$. B. Japan.

c. Ciccaba, Wagl. 1832.

329. Syrnitu Woodfordit.

B. MI. Athene Woodfordii, A. Smith, Ill. S. Afr. Zool. pl. 71. Noctua Woodfordii, A. Smith. Syrnium Woodfordii, Cass. Syrnium (Ciccaba) Woodfordii, Kaup.

S. Africa. 
330. Syrnium HUHULA.

B. M.

Strix huhula, Daud. Levaill. Ois. de l'Afr, t. 41. S. lineata, Shaw. S. albomarginata, Spix. Ciccaba hubula, Wagl. Athene lineata et A. huhula, G. R. Gr. Surnia lineata et S. huhula, Less. Noctua lineata, Cuv. Syrnium Ciccaba) hubula, Kaup.

S. America (Cayenne).

331? Syrnium cayanensis.

Strix cayanensis, Gm. Pl. enl. 442. Shaw, Nat. Misc. pl. 477. Athene et Strix cayanensis, G. R. Gr. Noctua cayanensis, Cuv. Syrnium? cayanensis, $\operatorname{Pr}, B$. Syrnium (Ciccaba) buhula, p. Kaup.

S. America (Cayenne).

332.? Syrnium fasciatum.

B. M. Strix fasciata, Vieill. Syrnium polygrammicum et S. zonocercus, G.R. Gr. S. fasciatum, Cass. ? Syrnium (Ciccaba) cayanensis et Syrnium (Ciccaba) buhula, p. Kaup.

S. America (Brazil, Caraccas).

333. Syrnium albitarse.

B. M. Syruium albitarse, G. R. Gr. Syrnium (Ciccaba) albitarse, Kaup.

S. America.

334. StrniUm hylophilum.

B. M.

Strix hylophila, Temm. $P l$. col. 373. Syrnium hylophilum, Less. Ulula fasciata, O. des Murs, Iconogr. Ornith. t. 27. Syrnium (Ciccaba) hylophilum, Kaup.

S. America (Brazil).

335. Syrnium grsella.

Ciccaba gisella, $\operatorname{Pr}$. B. Consp. p. 44.

S. America (Brazil).

336. Syrnium geuamulatum.

Strix squamulata, Licht. Syrnium squamulatum, $\operatorname{Pr}, B$. Mexico. 
337. Syrnium virgatum.

Syrnium virgatum, Cass.

Mexico?; S. America (Cayenne, St. Fé de Bogota) and Trinidad.

338. SyRnIUM MACABRUM.

Syrnium macabrum, Pr. B. Consp. p. 53.

S. America.

339. SyrniUm albigulare.

Syrnium albo-gularis, Cass.

S. America.

340. Syrnidm Lathami.

Strix Lathami, Pr. B. Athene Lathami, G. R. Gr. Ciccaba Lathami, $\operatorname{Pr} . B$.

b. Pulsatrix, Kaup, 1849.

341. Syrnium torquatum.

B. $M$.

Strix torquata, et S. personata, Daud. Levaill. Ois. de l Afr. $t$. 42, 44. S. superciliosa, et S. larvata, Shaw. Nat. Misc. pl. 801 S. pulsatrix, Pr. Max. S. perspicillata, Lath. Gen, Syn. pl. 107. Noctua torquata, Cuv. Syrnium personatum. G. R. Gr. S. pulsatrix, Pr. Max. Athene torquata, G. R. Gr. Ciccaba torquata, Pr. B. C. perspillata, Cass. Syrnium (Pulsatrix) torquatum, Kaup.

S. America, (Guiana, Cayenne, Surinam, Brazil, and Bolivia).

342. Syrnium Meianotum.

Noctua melanota, Tschudi, Fauna Per. t. 4. Athene melanota, Pucher. Ciccaba melanonota, Pr. $B$.

S. America (Brazil, Peru).

e. Bulaca, Hodgs. 1837.

Meseidus, Hodgs. 1841.

343. Syrnium indranee.

Strix indranee, Sykes. U. M. sis, Hodys. Ulula? newarensis, et Bulaca newarenG. R. Gr. Bulaca monticola, Jerd. Syrnium indranee, India. G. R. Gr. Syrnium (Bulaca) indranee, Kaup. 
344. Syrnium sinense.

B. M.

Strix sinensis, Lath. Gr. Ill. Ind. Zool. pl. 21. S. orientalis, Shaw. Otus sinensis, Steph. Bulaca sinensis, Jerd. Syrnium sinensis, G. R. Gr. S. ocellatum, Less. Syruium (BuIndia. laca) sinense, Kaup.

345. Syrnium seloputo.

Strix seloputo, Horsf. S. pagodarum, Temm. Pl. col. 230. Syruium pagodarum, Cuv. S. seloputo, G. R. Gr.

Syrnium (Bulaca) pagodarum, Kaup. Java.

346. Syrnidu leptogramicti.

Strix leptogrammicum, Temm. Pl. col.525. S. leptogrammica, var. Schl. Syrnium leptogrammicum, Cass. Phodilus leptogrammicus, Less. Ciccaba leptogrammica, et C. myrtha, $P r . B$.

Malayan Archipelago (Borneo, Sumatra).

347. Strnium humeralis.

? Hombr. \&. Jacq. Voy. au Pole Sud. Ois. t. 4, f. 1. Athene, sp. 40, G. R. Gr. Athene humeralis, $\operatorname{Pr}, B$.

Malayan Archipelago.

\section{Otus.}

a. Otus, Cuv. 1799-1800.

Egolius, Keys. et Bl. 1840.

Asio, Sw. 1831.

Nyctalops, Wagl. 1832.

348. Otug vulgaris.

B. M.

Strix otus, L. Pl. enl. 22. Gould, B. of Eur. pl. 39. S. soloniensis, Gm. Otus vulgaris, Flem. O. asio, Leach. 0. europæus, Steph. O. communis, Less. O. sylvestris, O. arboreus, et $\mathrm{O}$. gracilis, Brehm. Bubo otus, Boie. Agolius otus, Keys. \& Bl. Asio otus, Margill. Yarr. Brit. B. 2 edit. i. fig. p. 124. Strix otus, Wils. Amer. Orn. pl. 51, f. 3. Audub. $\bar{B}$. of Amer. pl. 383. Otus americanus $(G m$.), $P r, B$. O. vulgaris, $A$ udub. O. Wilsonianus, Less. O. Wilsonii, et O. stygius, G. R. Gr. Archiv. du Mus. iv.t. 24. O. communis, var. brasiliensis, Less. Strix (Asio) utus, Rich. \& Sw. Nyctalops stygius, Wagl.

Great Britain; Europe, Siberia, N. A frica, and N. America. 


\section{b. Pseudoscops, Kaup, 1849.}

349. Ötus Gramaricus.

B. M.

Ephialtes grammicus, Gosse, B. of Jam. pl. 19. Otus (Pseudoscops) grammicus, Kaup. Scops grammicus, $\operatorname{Pr} . B$.

Jamaica.

350. Otus SIguapa.

Otus siguapa, D'Orb. Voy. IIsle de Cuba, t. 2.

Cuba.

c. Rhinostrix, Kaup, 1849.

351. Otus americanus.

B. M.

Strix americanus, et S. mexicanus, Gm. S. longirostrix, Spix, Av. Bras. t. 9, a. S. maculosa, Pr. Max. Bubo clamator, Vieill. Ois. de l'Amer. Sept. t. 20. Otus mexicanus, Cuv. Otus (Rhinostrix) americanus, Kaup. Audub. B. of Amer. pl. 412 .

352. Otus Madagascariensis.

B. M. Otus madagascariensis, $A$. Smith. Otus (Rhinostrix) madagascariensis, Kaup. Bubo madagascariensis, Pr. B. Achiv. du Mus. iv. t. 23.

Madagascar.

\section{d. Brachyotus, Gould, 1837.}

353. OtUs BRAchyotus.

B. M.

Strix brachyotus, Gm. Gould, B. of Eur. pl. 40. S.ægolius, S. ulula et S. accipitrina, Pall. S. caspia, Shaw. S. aretica, Sparm. S. tripennis, Shrank. S. palustris, Smies. S. brachyura, Nils. Strix (Asio) brachyotus, Rich. \& Sw. Wils. Amer. Orn. pl. 33, f. 3. Audub. B. of Amer. pl. 410. Otus ulula, Cuv. O. palustris et 0 . agrarius, Brehm. O. brachyotus, Boie. O. microcephalus, Leach. Brachyotus palustris, Gould. Egolius brachyotus, Keys \& $B l$. Asio brachyotus, Macgill. Yarr. Brit. B. 2 edit. i. fig. p. 128.

Great Britain; Europe, Asia, N. Africa, N. and S. America and Hawiian Islands. 
354? Otus galapagoensis.

B. M.

Otus (Brachyotus) galapagoensis, Gould, Voy. of Beagle, pl. 3.

Islands of Galapagos.

e. Phasmaptynx, Kaup, 1849.

355. Orus capensis.

B. $M$.

Otus capensis, A. Smith, Ill. S. Afr. Zool. pl. 67. O. abyssinicus, Guer. Ferr. et Gal. Voy. Abyss. t. 3. Otus (Phasmaptynx) capensis, Kaup. Otus brachyotus et Brachyotus helvolus, Licht.

S. and N.-E. Africa.

iv. Strigine.

Striginæ, Pr. B. 1838-49-50; G. R. Gr. 1840-41-45; Bl. 1849 ; Kaup, 1849.

Strigina, p. Vig. 1825.

1. Strix.

a. Strix, $L .1735$.

Aluco, Flem. 1822.

Hybris, Nitzsch. 1840.

Stridula, Sel. Longch. 1842.

356. Strix flammea.

B. M.

Strix flammea, L. Pl. enl. 440. Gould, B. of Eur. pl, 36.

S. guttata, Brehm. Aluco flammeus, Flem. Yarr. Brit. B.

2 edit. i. fig. p. 133. Strix pusilla et S. parva, $B l$. ?

Great Britain ; Europe, N. Africa.

357? STRIX POENSIS.

Strix poensis, Fras.

Fernando Po.

358. ? Strix delicatula.

B. M.

Strix flammea, Vig. \& Horsf. S. delicatula, Gould, B. of Austr. pl. 31.

Australia (except Van Diemen's Land.) 
359.? Strix LULU.

B. $M$.

Strix lulu, Peale, U. S. Expl. Exp. viii. p. 74, pl. 21.

Samoan Group (Opolu).

360.? Strix Jafanica.

B. $M$.

Strix flammea, Hodgs. Bl. S. jaranica, De Wurmb. S. flammea, var. c. Kaup.

Java and India.

361. ? Strix pratincola.

B. $\mathbf{M}$. Strix flammea, Wils. Amer. Orn. pl. 50, f. 2. S. americana, Audub. B. of Amer. pl. 171. S. pratincola, Pr. B. S. flammea, var. b. Kaup. S. perlata, p. G. R. Gr.

N. America.

362. Strix punctatissima.

Strix punctatissima, G. R. Gr. Voy. Beagle, pl. 4.

Islands of Galapagos.

363. Strix personata.

B. M.

Strix personata, Vigors. S. cyclops, Gould, B. of Austr. pl. 29. Australia (except Van Diemen's Land).

364. Strix castanops.

Strix castanops, Gould, B. of Austr. i. pl. 28.

Van Diemen's Land.

b. Glyphidiura, Reichenb. 1850.

365. Strix perlata.

B. M.

Strix perlata, Lichst. S, furcata, Temm. Pl. col. 432.

S. America and Island of Cuba.

c. Glaux, $B l .1848$.

366. Strix candida.

B. $\mathbf{M}$.

Strix candida, Tick. S. longimembris, Jerd. S. capensis, A.

Smith, Ill. S. Afr. Zool. pl 45. Glaux javanica (?) Bl.

India and S. Africa. 
d. Megastrix, Kaup, 1849.

367. Strix tenebricosA.

B. M.

Strix tenebricosa, Gould, B. of Austr. i. pl. 30. Strix (Megastrix) tenebricosa, Kaup.

New South Wales.

e. Pholidus, J. Geoffr. 1830.

368. Strix BAdis.

B. $\mathrm{M}$.

Strix badia, Horsf. Zool. Res. pl. Pl. col. 318. Pholidus badius, J. Geoffr. Strix (Pholidus) badius, Kaup.

Java and Nepal. 


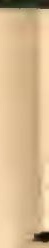

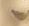

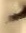




\section{Order II. PASSERES.}

Passeres, Linn. 1735 ; Lath. 1790 ; Cur. 1795-1796.

Picæ, p. Linn. 1735; Lath. 1790.

Hymenopodes, Mochring, 1752.

Scansores, p. Cur. 1799-1800; Illig. 1811; Cabanis, 1847.

Ambulatores, Illig. 1811.

Chelidones-Alcyones-Coraces-Canori-Passerini-Scansores, p. Temm. 1815 .

Sylvicolæ, anisodactyli-S. zjgodactyli, p. Vieill. 1816.

Anisodactrli-Insectirores-Omnirores-Granirores, Temm. 1820. Passeri, Ranz. 1823.

Insessores, Vigors, 1825.

Scansores, p.-Oscines, Keys. \&. Bl. 1840.

Strisores-Clamatores, p. Cabanis, 1847.

Ornitbes, p. Kaup, 1844.

\section{Tribe 1. FISSIROSTRES.}

Passeres, p. Linn. 1735 ; Mahr. 1752.

Picr, p. Linn. 1735.

Accipitres, p. Mrchr. 1752.

Plumipedes, p.-Fissipedes isodactyles-F. anisodactyles, conicosubulirostres, p.-Anomalipedes, p. Schaff. 1774.

Scutipedes, p.-Negliges, p.-Brevipedes, Scop. 177.

Pice, p. Lalh. 1790.

Planirostres - Tenuirostres, p. - Cuneirostres, p.-Lerirostres, p. Cuv. 1799-1800.

Hiantes-Coraces, p.-Canori, p.-Dentirostres, p.-Serrati, p.Amphiholi, p.-Angulirostres-Syndactsli, Illig. 1811.

Chelidones-Alciones, Temm. 1815.

Chelidones-Baccirori, p.-Prionites, p.-Barbati, p.-Pelmatodes -Aureoli, Vieill. 1816.

Fissirostres-Syndactyles-Scansores, p.-Conirostres, p.-Dentirostres, p. Cuv. 1817.

Omnirores, p.-Insectirores, p.-Z Zggodactyli, p. Temm. 1820.

Pogonofori, p.-Sindactili - Pelmatodi-I falti, p.-PlereoramfiEuristomi, p.-Chelidonii-Piezoramfi, Ranz. 1823. 
Fissirostres-Conirostres, p.-Scansores, p. Vigors, 1825.

Heterodactyles, p. Less. 1831.

Gregarii, p.-Serrati, p.-Sagittilingues-Amphiboli, p.-Angulirostres, Pr. Bonap. 1831.

Oscines, p.-Scansores, p. Keys. \& Bl. 1840.

Volitatrices-Jaculatrices-Excurtrices, p. Macgill. 1840.

Ornithes fissirostres, p.-O. syndactyli, p. Kaup, 1844.

Macrochires-Oscines, p.-Clamatores, p. Cabanis, 1847.

\section{Sub-Tribe 1. FISSIROSTRES NOCTURNAE.}

\section{CAPRIMULGID $\approx$.}

Caprimulgidæ, Vig. 1825 ; Sw. 1831-37; G. R. Gr. 1840-4146 ; Cab. 1847 ; Pr.B. $1849-50 ;$ Bl. 1849.

Hirundinidæ, p. et Ámpelidæ, p. Pr. B. 1831.

Caprimulgides, Sundev. 1835.

\section{i. Steatornithine.}

Steatorninæ, G. R. Gr. 1846; Cab. 1847.

Steatornithinæ, Pr. B. $1849-50$.

Podarginæ, G.R. Gr. 1840-41; Cab. 1847 ; Bl. 1849.

\section{Steatornis.}

Steatornis, Humb. 1817.

369. Steatornis caripennis.

B. M.

Caprimulgus steatornis et Steatornis caripennis, Humb. Obs. Znol. t. 44. Ann. du Mus. 1834, t. 15. Nyctibius? steatornis, Steph.

Trinidad and S. America (Sta. Fé de Bogota). 


\section{Podargus.}

a. Podargus, Cuv. 1829.

370. Podargus humeralis.

B. M.

Podargus humeralis, Vig. \& Horsf. Jard. \& Selby, lll. of Orn.

pl. 88.? Gould, B. of Austr. ii. pl. 3. P. australis et P.? gracilis Steph.? P. cinereus, Cuv. P. strigoïdes, G. R. Gr.? Caprimulgus gracilis et C. strigoïdes, Lath.?

New South Wales.

371. Podargus Cuvieri.

B. M.

Podargus Cuvieri, Vig. \& Horsf. Gould, B. of Austr. ii. pl. 4.

South Australia and Van Diemen's Land.

372. Podargus brachypterus.

Podargus brachypterus et P. macrorhynchus, Gould.

S. and W. Australia.

373. Podargus phalenoides.

B. M.

Podargus phalænoïdes, Gould, B. of Austr. ii. pl. 5.

N. Australia.

374. Podargus plumiferus.

Podargus plumiferus, Gould, B. of Austr. ii. pl. 6.

New South Wales.

375. Podargus megacephalus.

Caprimulgus megacephalus, Lath. Podargus megacephalus, G.R.Gr. P. Stanleyanus, Vig. \& Horsf.

New South Wales.

376. Podargus ocellatus.

Podargus ocellatus, Quoy \& Gaim. Voy. de l'Astrol. Ois. t. 14.

New Guinea.

377. Podargus papdensis.

Podargus papuensis, Quoy \& Gaim. Voy. de l'Astrol. Ois. t. 13.

Less. Compl. Buff. Ois. t. 8, f. 1.

New Guinea. 
b. Batrachostomus, Gould, 1838.

Bombycistomas, Hay, 1841.

378. Podargus javanensis.

B. M.

Podargus javanensis, Horsf. Zool. Res. pl. P. cornutus, Temm. Pl. col. 159. Steph. Gen. Zool. xiii. pl. 41. P. stellatus, Gould. Batrachostomus javanensis, Gould. B. stellatus, G. R. Gr.

Malacca and Java.

379? Podargus affinis.

Batrachostomus affinis, $B l$.

Darjeeling.

380. Podarges moniliger.

Batrachostomus moniliger, Layard, Journ. As. Soc. Beng. 1850, p.

Ceylon.

381. Podargus parvulus.

Podargus parvulus, Temm. Batrachostomus parvulus, $\operatorname{Pr} . B$. Consp. p. 57.

Malayan Archipelago.

382. Podargus crinifrons.

Podargus crinifrons, Temm. Batrachostomus crinifrons, $\operatorname{Pr}$. B. Consp. p. 57.

Malayan Archipelago.

383. Podargus auritus.

B. $M$.

Podargus auritus, Vigors, Griff. An. Kingd. ii. pl.p. 114. P. Fullertonii, Bl. Batrachostomus auritus, Gould, Icon. Av. pl. Bombycistomas Fullertonii, Hay.

Malayan Peninsula and Archipelago. 


\section{Nyctibius.}

a. Nyctibius, Vieill. 1816.

Nyctornis, Nitzsch, 1840.

384. Nyctibius gRandis.

B. $\mathrm{M}$. Caprimulgus grandis, $G m . \quad P l . e n l .325 . \quad$ C. maximus, Shaw, Lev. Mus. pl. 9. Nyctibius grandis, Vieill. Jard. \& Selby, Ill. Orn. pl. 89. Gr.\&. Mitch. Gen. of B. pl. 16.

S. Anserica (Cayenne).

385. Nyctibius æthereus.

B. $\mathbf{M}$ 。

Caprimulgus æthereus, $\operatorname{Pr}$. Max. C. longicaudatus, Spix. $A v$. Bras. t. 1. Nyctibius æthereus, G. R. Gr. N. longicaudatus, Lafr.

S. America (Brazil, Paraguay, Peru).

386. Nyctibius cornutus.

Caprimulgus cornutus, Vieill. Nyctibius cornutus, G.R. Gr. N. urutau, Lafr.

S. America (Brazil, Paraguay, La Plata and Peru).

387. Nyctibius Jamaicensis.

B. M.

Caprimulgus jamaicensis, Gm. Lath. Gen.Syn.pl.57. Gosse, B. of Jam. pl. 8. Nyctibius jamaicensis. Steph.

Jamaica.

388. ? Nyctibius pectoralis.

B. M.

Nyctibius pectoralis, Gould, Icon. Av. pl.

S. America (Cayenne).

389? Nyctibius paltidus.

Nyctibius pallidus, Gosse, B. of Jam. pl. 7 .

Jamaica.

390. Nyctibius Bracteates.

Nyctibius bracteatus, Gould.

S. America (Sta. Fé de Bogota). 
391. Nyctibies Rufus.

Nyctibius rufus, Cab. R. Schomb. Reis. iii. p. 711.

S. America (British Guiana).

b. Selochusa, G. R. Gr. 1841.

392. Nyctibius forficatus.

Caprimulgus forficatus, Vieill. Levaill. Ois. de l Afr. t. 47, 48. C. furcatus, Cuv. Nyctibius forficatus, Lafr. Selochusa forficata, G.R. Gr.

S. Africa.

\section{Agotheles.}

Egotheles, Vig. \& Horsf. 1825.

393. Egotheles NOYE HollaNdie.

B. M.

Caprimulgus novæ hollandiæ et C. vittatus, Lath. C. cristatus, Shaw, Phil. Bot. Bay, pl. p. 270. Egotheles novæ hollandiæ, Vig. \&. Horsf. Gould, B. of Austr. ii. pl. 1. B. cristatus, G. R. Gr. E. lumulatus, Jard \& Selby Ill. of Orn. pl. 149. E. australis, Sw. Podargus? novæ hollandiæ, Steph. Mag. de Zool. 1837. Ois.1. 82. Lath. Gen. Syn. Suppl. ii. pl. 136.

Australia (except the North).

394. Egotheles LevCogaster.

Egotheles leucogaster, Gould, B. of Austr. ii. pl. 2.

B. M.

N. Australia.

\section{ii. Caprimulgina.}

Caprimulginæ, Sw. 1831 ; Pr. B. $1831-49-50 ;$ G. R. Gr. $1840-$ $41-47$; Cab. $1847 ;$ Bl. 1849.

\section{Caprimulgus.}

a. Caprimulgus, $L$. 1756 .

Nyctichelidon, Renn. 1831.

395. Caprimulgus europerus.

B. M.

Caprimulgus europæus, $L . \quad P l$. enl. 193. C. punctatus, Mey.

Brehm. C. maculatus, Brehm. Nyctichelidon europæus, Renn.

Gould, B. of Eur. pl. 51. Naum. Vög. t. 148.

Great Britain; Europe and N. Africs. 
396. Caprimulgus ruficollis.

B. M.

Caprimulgus ruficollis, Temm. C. rufitorques, Vieill. Gould, B. of Eur. pl. 52 .

S.-W. Europe and N. Africa.

397. Caprimulgus Smithit.

B. M.

Caprimulgus europæus, A. Smith, Ill. S. Afr. Zool. Birds, pl. 102. C. Smithii, $\operatorname{Pr}$. $B$.

S. Africa.

398. Caprimulgus rufigena.

B. M.

Caprimulgus rufigena, A. Smith, Ill. S. Afr. Zool. Birds, pl. 100 .

S. Africa.

399. Caprimulgus lentiginosus.

B. M.

Caprimulgus lentiginosus, A. Smith, Ill. S. Afr. Zool. Birds, pl. 101 .

S. Africa.

400. Caprimulgus natalensis.

B. M.

Caprimulgus natạlensis, A. Smith, Ill. S. Afr. Zuol. pl. 99.

S. Africa, (Natal).

401. Caprimulgus pectoralis.

B. M. Caprimulgus pectoralis, Cuv. C. africanus, Steph. Levaill. Ois. de l'Afr. t. 49.

S. Africa.

402. Caprimulgus tristigma.

Caprimulgus tristigma, Rüpp. Vög. N. Ost. Afr. t. 3. C. poliocephalus, Rüpp.

Abyssinia.

403. Caprimulgus poliocephalús.

Caprimulgus poliocephalus, Rïpp. V̈̈g. N. Ost. Afr. t. 4. C. tetrastigma, Rüpp.

Abyssinia. 
404. Caprimulgus infuscatus.

Caprimulgus infuscatus, Rüpp. Zool. Atl. t. 6. C. nubicus, Licht.

Nubia.

405. Caprimulgus isabellinus.

Caprimulgus isabellinus, Temm. Pl. col. 379. C. ægyptius, Licht.

N.-E. Africa.

406. Caprimulgus extmius.

Caprimulgus eximius, Rüpp. Pl. col. 398.

N.-E. Africa.

407. Caprimulgus concretus.

Caprimulgus concretus, Temm. Pr. B. Consp. p. 60.

Ashantee.

408. Caprimulgus madagascariensis.

Caprimulgus madagascariensis, Syanz.

Madagascar.

409. Caprimulges indicus.

Caprimulgus indicus, Lath.? C. cinerascens, Vieill. C. saturior, Hodgs. Gr. Ill. Ind. Zool. pl. 34, f. 1. C. europæus, Raff.

India and Malacca.

410. Caprimulgus albonotatés.

B. M.

Caprimulgus albonotatus, Tick. C. nipalensis et C. innotatus, Hodgs. C. macrourus et C. gangeticus, $B l$.

North and Central India.

411. Caprimulgus macrourus.

Caprimulgus macrourus, Horsf. Eurostopodus macrourus, $G$. R. Gr. Gould, B. of Austr. ii. pl. 9.

Java and N. Australia. 
412. Caprimulgus spilocercus.

B. M.

Caprimulgus macrourus et C. indicus, Jerd. Ill. Ind. Orn. pl. 24. C. mahrattensis, Jerd. C. spilocercus, G. R. Gr.

Madras.

413. Caprimulgus bimaculatus.

Caprimulgus bimaculatus, Peale, U. S. Expl. Exped, viii. p. 170, pl. 48 , f. 1.

Malayan Peninsula.

414. Caprimulgus monticola.

B. M. Caprimulgus monticola, Frankl. C. gymnopus, Hodgs. C. arenarius, $\boldsymbol{B} l$.

India and Sumatra.

415. Caprimulgus affinis.

Caprimulgus affinis, Horsf. C. pallidus, $G r$.

B. M. Java.

416. Caprimolgus asiaticus.

B. M.

Caprimulgus asiaticus, Lath. Gr. Illustr. Ind. Zool. pl. 34, f. 2. India.

417. Caprimulgus mahrattensis.

C. mahrattensis, Sykes. C. atripennis, Jerd.? Gould, B. of Asia, pl.

S. India and Ceylon.

418. Caprimolgus jotaka.

Caprimulgus jotaka, Temm. \&. Schl. Fauna Jap. Ois. t. 12, 13. Japan.

419. Caprimulgus icteropterus.

Caprimulgus icteropterus, Vieill.

China.

420. Caprimulgus binotatus.

Caprimulgus binotatus, Temm. Pr. B. Consp. p. 60. Borneo. 
421. Caprimulges arondinaceus.

Caprimulgus arundinaceus, $\operatorname{Pr} . B$. Hombr. \& Jacq. Voy. au Pole Sud. t. 21, f. 2.

422. Caprimulgus cayanensis.

B. $\mathrm{M}$.

Caprimulgus cayanensis, $G m$. Pl. enl. 760. C. cayanus, Lath.

C. leucurus, Vieill. C. leopetes, Jard. \& Selby, Ill. of Orn.

pl. 87. Antrostomus cayanensis, Pr. B. Shaw, Nat. Misc. pl. 1045.

S. America (Cayenne).

423. Caprimulgus bifasciatus.

B. M.

Caprimulgus bifasciatus, Gould. Antrostomus bifasciatus, Pr. $B$.

S. America (Chili).

424. Caprimulgus feurcauda.

Caprimulgus æquicauda, Peale, U. S. Expl. Exped. viii, p. 168 , pl. 47, f. 1 .

S. America (Peru).

425. Caprimulgus ocellatus.

B. M. Caprimulgus ocellatus, Tschudi. Fauna Per. t. 5, f. 2. "C. brasiliensis, Gm." Tschudi. Antrostomus ocellatus, Pr. B.

S. America (Peru).

426. Caprimulgus nigrescens.

Caprimulgus nigrescens, Cab. R. Schomb. Reis. iii. p. 710.

S. America (British Guiana).

427. Caprimulgus vocifer.

Caprimulgus vociferus, Wils. Amer. Orn. pl. 41. C. clamator, Vieill. Ois, de l'Amer. Sept. t. 23. Audub. B. of Amer. pl. 82. Antrostomus vocifer, Pr. B. Shaw, Nat. Misc. pl. 1053.

N. America (South and Central Parts).

428. Caprimulgus dominiaus.

Antrostomus dominicus, $\operatorname{Pr}, B$. Consp. p. 61 .

St. Domingo. 
429. Caprimulgus californianus.

Antrostomus californianus, $\operatorname{Pr} . B$. Consp. p. 61.

California.

430. Caprimulgus Nuttalir.

Caprimulgus Nuttalii, Audub. B. of Amer. pl. 495.

Califurnia.

431. Caprimulgus guianensis.

Caprimulgus guianensis et C. griseus? Gm. Pl.enl.733. C. variegatus, Vieill. C. jaspideus, Merr. Antrostomus guianensis, $P r$. $B$.

S. America (Guiana, La Plata, Patagonia).

432. Caprimulgus Mitchellit.

B. M.

Caprimulgus semitorquatus, G. R. Gr. \& Mitch. Gen. of $B$. pl. 17.

S. America (Para).

433. Caprimulgus odonpteron.

Caprimulgus odonpteron, Less. Antrostomus odon pteron, $\operatorname{Pr} . B$.

Antilles.

434. Caprimulgus parvulus.

Caprimulgus parvulus, Gould. Antrostomus parrulus, $\operatorname{Pr}, B$. S. America (La Plata).

435. Caprimolgus decussatus.

Caprimulgus decussatus, Tschudi, Fauna Per. t. 5. f. 1. Antrostomus decussatus, $P r . B$.

S. America (Peru).

436. Caprinulgus longirostris.

Caprimulgus longirostris, $P r . B$. Antrostomus longirostris, Pr. B.

S. America.

437. Caprimulgus sphenurus.

Caprimulgus sphenurus, Vieill C. Azaræ, Merr.

S. America (Paraguay). 
438.? Caprimulgus torquatus.

Caprimulgus torquatus, Gm. Will. Orn.t. 14.

S. America (Brazil).

439. Caprimulgus macroniystax.

Caprimulgus macromystax, Wagl.

Mexico.

440. Caprimulgus conterminus.

Caprimulgus conterminus, Peale, U. S. Expl. Exped. viii. p. 169. pl. 47, f. 2.

S. America (Chili).

b. Eucapripodus, Less. 1843.

441. Caprimulgus albicollis.

Caprimulgus albicollis, Gm. Eucapripodus albicollis, Less. Antrustomus albicoliis, $\mathrm{Pr}$. $\mathrm{B}$.

S. America (Cayenne, Paraguay, and Bolivia).

c. Antrostomus, Gould, 1838.

442. Caprimulgus carolinensis.

B. N.

Caprimulgus carolinensis, Wils. Amer. Orn. pl. 54, f. 2 . C. rufus, Vieill. Ois. d'Amer. Sept. t. 25? Audub. B. of Amer. pl. 52. Antrostomus carolinensis, Gould.

N. America.

443. Caprimulgus serico-caudatus.

Antrostomus serico-caudatus, Cass. Proc. Ac. Nat. Sc. Phil. iv. p. 236.

S. America.

d. Hydropsalis, Wagl. 1832.

Psalurus, Sw. 1837.

444. Caprimulaus furcifer.

Caprimulgus furcifer, Vieill. C. fissicaudus, Merr. Hydropsalis Azaræ, Wagl. H. furcifer, G. R. Gr.

S. America (Brazil, Paraguay). 
445. Caprimulgus psalurus.

Caprimulgus psalurus, Temm. Pl. col. 157. Hydropsalis psalurus, $P r . B$. H. furcifer, p. G. R. $G r$. Psalurus macropterus, $S w$.

S. America (Brazil).

446. Caprimulgus creager.

Hydropsalis creagra, $\operatorname{Pr}$. B. Consp. p. 58. H. limbatus, Cass.

S. America (Brazil).

447. Caprinulgus lixa.

Hydropsalis lyra, Gould. Pr. B. Consp. p. 59. H. segmentatus, Cass.

S. America (Sta. Fé de Bogota).

448. Caprimulgus manurus.

B. M.

Caprimulgus manurus, Vieill. Caprimulgus (Hydropsalis) manurus, G. R. Gr. Hydropsalis manurus, $\operatorname{Pr}$. $B$.

S. America (Brazil).

449. Caprimulgus Climacocercus.

B. M.

Caprimulgus climacocercus, Tschudi, Fauna Per. t. 6, f. 1. Caprimulgus (Hydropsalis) climacocercus, G. R. Gr. $\mathrm{Hy}-$ dropsalis climacocercus, $\operatorname{Pr} . B$.

S. America (Peru, British Guiana).

450. Caprimulgus trifurcatus.

Hydropsalis trifurcatus, $\operatorname{Pr}$. B. Consp. p. 59. Caprimulgus trifurcatus, Natt.

S. America (Peru).

e. Tetroura, Less. 1843.

451. Caprimulgus enicurus.

Caprimulgus enicurus, Vieill. C. cordicilla, Merr. Tetroura enicura, Less.

S. America. 


\section{Nyctidromus.}

Nyctidromus, Gould, 1838.

452. Nyctidromus Derbyanus. Nyctidromus Derbyanus, Gould, Icon. Av. pl.

B. M.

S. America (Brazil, Para).

453.? Nyctidrouus Grallarius.

Caprimulgus grallarius, $\operatorname{Pr}$. Max. Nyctidromus grallarius, $\operatorname{Pr}$. B. Consp. p. 62.

S. America.

454. NyCTIDROMUS AFFINIS.

Nyctidromus affinis, $G . R . G r$.

B. M.

Mexico and Honduras.

\section{Eleothreptus.}

Eleothreptus, G. R. Gr. 1840.

Amblypterus, Gould, 1837.

455. Eleothreptus anomalos.

B. M. Amblypterus anomalus, Gould, Icon. Av. pl. Eleothreptus anomalus, G. R. Gr.

Demerara.

\section{Eurostopodus.}

a. Eurostopodus, Gould, 1837.

456. Eurostopodu's albogularis.

Caprimulgus albogularis, Vig. \& Horsf. C.mysticalis, Temm. $\mathrm{Pl}$. col. 410. C. albomaculatus, Cuv. Eurostopodus albogularis, Gould, Be of Austr. ii. pl. 7.

New South Wales.

457. Eurostopodus guttatus.

B. M. Caprimulgus guttatus, Vig. \& Horsf. Eurostopodus guttatus, Gould, B. of Austr. ii. pl. 8.

Australia (except Van Diemen's Land). 
b. Lyncornis, Gould, 1838.

458. Eurostopodus CERVINICEPS.

Lyncornis cerviniceps, Gould, Icon. Av. pl. Eurostopodus cerviniceps, G. R. Gr.

Burmah.

459. Eurostopodus macrotis.

B. $\mathrm{M}$.

Caprimulgus macrotis, Vigors. Lyncornis macrotis, Gould, Icon. $A v$. pl. . Eurostopodus macrotis, G. R. Gr.

Philippine Islands.

460. Eurostopodus Temminckir.

B. M.

Lyncornis Temminckii, Gould, Icon. Av. pl. Caprimulgus pulcher, A. Hay. C. imberbis, Temm. Eurostopodus Temminckii, G. R. Gr.

Malacca and Java.

461. Eurostopodus macropterus.

Lyncornis macropterus, Temm. Pr. B. Consp. p. 62.

Celebes.

\section{Chordeiles.}

Chordeiles, Sw. 1831.

462. Chordeiles Wiederspergit.

Caprimulgus Wièderspergii, Reichenb. All. Deuts. Naturh. $Z$ eit. 1846, t. 5. Eurostopodus? Wiederspergii, G. R. Gr. Scotornis climacurus? Pr. B.

463. Chordeiles virginianus.

B. $\mathrm{M}$.

Caprimulgus virginianus, $G m$. C. popetue, Vieill. Ois, de l'Amer, t. 24. C. americanus, Wils. Amer. Orn. pl. 40. Audub. B. of Amer. pl. 147. Chordeiles virginianus, Sw. Caprimulgus (Chordeiles) virgivianus, Sw. \&.Rich.

N. America.

464. Chordeiles sapiti.

Caprimulgus sapiti, Natt. Chordeiles sapiti, Pr. B. Consp. p. 63.

S. America. 
465. Chordeiles americanus.

Caprimulgus americanus, $L$. Sloane, Jam. pl.255, f. 1. Chordeiles americanus, G. R. $G r$.

S. America.

466. Chordeiles semitorquatus.

Caprimulgus semitorquatus, $\mathrm{Gm}$. Pl. enl. 734. Antrostomus semitorquatus, $\operatorname{Pr}$. $B$.

S. America (Cayenne)

467. Chordeiles brasitrensis.

Caprimulgus brasiliensis, Gm. Petiv. t. 59, f. 1. C. noitibo, Vieill. Chordeiles brasiliensis, $\operatorname{Pr} . B$.

S. America (Brazil, Peru).

468. Chordeiles actitipennis.

Caprimulgus acutipennis, Bodd. C. acutus, Gm. Pl.enl.732.

S. America.

469. Chordeiles rupestris.

B. M.

Caprimulgus rupestris, Spix, Av. Bras. t. 2. Chordeiles rupestris, G. R. Gr.

S. America (Brazil, Para, Bolivia).

470. Chordeiles labeculatus.

Chordeiles labeculatus, Jard.

Bermuda.

471. Chordeiles hirundiaceus.

Caprimulgus hirundinaceus, Spix, $A v$. Bras. t. 3, f. 1. Chordeiles hirundinaceus, $P r, B$.

S. America (Brazil).

472. Chordeiles ledCopterus.

Caprimulgus leucopterus, $\operatorname{Pr}$. Max. C. leucopygius, Spix, Av. Bras. t. 3, f. 2. Chordeiles leucopterus,

S. America (Brazil). 
473. Chordeiles exilis.

Caprimulgus exilis, Less. C. pruniosus, Licht. Fauna Per. t. 6, f. 2. Chordeiles exilis, $\operatorname{Pr}, B$.

S. America (Chili).

474. Chordeiles peruvianis.

Chordeiles perurianus, Peale, U. S. Expl. Exped. viii. p. 172, pl. 48, f. 2 .

Peru.

475. Chordeiles minutus.

Caprimulgus minutus, Natt. Chordeiles minutus, $P r$. B. Consp. p. 63.

S. America (Brazil).

iii. Podagerinete.

Podagerinæ, G. R. Gr. 1847.

Scotorninæ, G. R. Gr. 1840-41,

Scotornithinæ, Pr. Bonap. 1849.

Caprimulginæ, p. Pr. Bonap. 1850.

\section{Scotornis.}

Scotornis, $S w .1837$.

476. Scotornis Cltmacurus.

Caprimulgus climacurus, Vieill. Gal. des Ois. t. 122. gicaudus, Steph. Scortornis climacturus, $S w$.

B. M.

C. lon-

N.-E. and W. Africa.

477.? Scotornis TRIMACULATUS.

Scotornis trimaculatus, $S w$.

W. Africa.

\section{Macrodipteryx.}

a. Macrodipteryx, $S w .1837$.

478. Macrodipteryx LongipenNis.

Caprimulgus longipennis, Shaw, Nat. Misc. pl. 265.

B. M. Af . Macrodipteryx africanus, Sw. B. of $W$. Afr. ii. pl. 5. M. longipennis, G. R. Gr.

N.-E. and W. Africa. 
b. Cosmetornis, G. R. Gr. 1840.

Semeïophorus, Gould, 1838.

479. MACRODYPTERYX VEXILLARIUS.

Semeïophorus vexillarius, Gould, Icon. Av. pl. . Cosmetornis vexillaria et Macrodipteryx vexillarius, G. R. Gr.

3. Podager.

Podager, Wagl. 1832.

Proïthera, Sw. 1837.

480. Podager nacunda.

B. $\mathrm{M}$. Caprimulgus nacunda, Vieill. C. diurnus, $\operatorname{Pr}$, Max. Pl. col. 182. C. campestris, Licht. Podager diurnus, Wagl. P. nacunda, G.R. Gr. Prö̈thera diurnus, $S w$.

S. America (Brazil, La Plata, Bolivia)

481. Podager Nattereri

B. M. Caprimulgus Nattererii, Temm. Pl. col. 107. Podager Nattererii, $G, R$. $G r$.

S. America (Brazil).

482. Podager Gouldir.

B. M.

Podager Gouldii, G. R. Gr. \& Mitch. Gen of B. pl. 18.

S. America (Cayenne).

483. Podager rufus.

Caprimulgus rufus, Bodd. Pl. enl. 735. C. brachypterus, Steph. Chordeiles? rufus, G. R. Gr. Antrostomus rufus, Pr. B.

S. America. 


\section{HIRUNDINID \&.}

Hirundinidæ, Leach. Vig. 1825 ; Pr.B.1831-49-50 ; Sw. 1831;

G.R. Gr. $1840-41-45 ; C a b .1847 ; B l .1849-50$.

Cypselidæ, Cab. 1847 ; Bl. $1849-50 ;$ Pr. B. 1850.

Hirundinides et Cypselides, Sundev. 1835.

Hemiprocnidæ, Streubel, 1848.

Macropteriginæ, $B l .1850$.

\section{i. Cypseline.}

Cypselinæ, Pr. B. $1838-49-50 ;$ G. R. Gr. 1840-41-45;

Cab. $1847 ; B l .1849$.

\section{Cypselus.}

a. Cypselus, Ill. 1811.

Apus, Scop. 1777.

Micropus, Wolf \& Mey. 1815.

Brachypus, Mey. 1815.

Pallenis, Reichenb. 1850.

484. Cypselus melia.

Hirundo melba, $L$. $E$. . A sus melba, Cuv. Less. A. alpinus, Less. Micropus melba et M. gutturalis, Boie. M. alpinus, Mey. \& Wolf. Cypselus melba, Ill. C. alpinus, Temm. C. gutturalis, Vieill. Gal. des Ois. t. 121. C. alpinus africanus, Temm. Gould, B. of Eur. pl.53, f. 2.

S. Europe, India, N. and S. Africa.

485. Cipselus pacificus.

Hirundo pacifica, Lath. Cypselus pacificus, G. R. Gr. C. australis, Gould, B. of Austr. pl. 11. Chætura pacifica, Steph. Acanthylis pacifica et Micropus australis, Boie.

New South Wales. 
486. Cypselos apus.

B. M.

Hirundo apus, $L$. Pl. enl. 242, f. 1. Gould, B. of Eur. pl. 53, f. 1. Cypselus apus, Ill. C. vulgaris, Steph. C. murarius, Temm. C. caffer, Licht. Micropus murarius, Mey. \&. Wolf. M. apus et M. caffer, Boie. Apus cafer, Less.

Great Britain ; Europe, W. Asia, N.; N.-E. and S. Africa.

487. Cypselus pygargus.

B. M. Cypselus pygargus, Temm. Pl. col. 460, f. 1. Atticora pygargus, Boie. Apus pygargus, Less.

S. Africa.

48. Cypselus vittatus.

Cypselus vittatus, Jard. \&. Selby, Illustr. Orn.n. s. pl.39. Micropus vittatus, Boie.

N.-W. Himalaya, China and Malayan Peninsula and Archipelago.

489. Cypselus unicolor.

B. M.

Cypselus unicolor, Jard. \&. Selby, Illustr. Orn. p1. 83. Micropus unicolor, Boie. Apus unicolor, Less.

Madeira.

490. Cypselus leucorrhous.

Hirundo leucorrhöus, Steph. Levaill. Ois. de l'Afr. t. 244, f. 1. H. nigra, Vieill. Cypselus leucorrhöus, Steph. Micropus lencorrhöus, Boie. A pus sinensis, Less.

S. Africa.

491. Cypselus abysinicus.

Cypselus abyssinicus, Licht. Isis, 1848, p. 354.

N.-E. Africa.

492. Cypselus affinis.

B. M. Cypselus affinis, Gr. Ill. Ind. Zool. pl. 35, f. 2. C. nipalensis Hodgs. C. montanus, Jerd. Micropus affinis, Boie.

India and Ceylon. 
493. Cypselus subforcatus.

Cypselus subfurcatus, Bl. C. affinis, var., Strickl.

Malacca.

494. Cypselús balassiensis.

B. M.

Cypselus balassiensis et C. palmarum, Gr. Ill. Ind. Zool. pl. 35, f. 1. Atticora palmarum, Boie.

India, Ceylon, and Malayan Peninsula.

495. Cypselus sinensis.

Hirundo sinensis, $\mathbf{G m}$. Cypselus sinensis, Cuv. Micropus sinensis, Boie.

India.

496. Cypselus ledconyx.

Cypselus leuconyx, $B l$.

India.

497. Cypselus andicolus.

B. M.

Cypselus andicolus, D'Orb. \& Lafr. Voy. dans l'Amer. Mer. Ois.

t. 42, f. 2. Micropus andicolus et Chelidon andicola, Boie.

S. America (Bolivia).

498. Cypselus montivagus.

Cypselus montivagus, D'Orb. \& Lafr. Voy. dans $I$ Amer. Mer. Ois. t. 42, f. 1. Acanthylis? et Cypselus montivagus, $G$. $R$.

Gr. Acanthylis noctivagus, Boie.

S. America (Bolivia).

499. CyPSElus SENEX.

B. M.

Cypselus senex, Temm. Pl. col. 397. Acanthylis? et Cypselus senex, G.R. Gr. Acanthylis senex, $\operatorname{Pr} . \vec{B}$. Pallene senex, Less. Hemiprocne senex, Streubel.

S. America (Brazil).

500. Cypselus fumigatus.

Cypselus fumigatus, Natt. Hemiprocne fumigata, Streubel, Isis, 1848 , p. 366.

S. America (Paraguay, Brazil). 


\section{c. Panyptila, $C a b .1847$.}

Pseudoprocne, Streubel, 1848.

501. Cypselus cayanensis.

B. $M$.

Hirundo cayanensis, Gm. Pl. enl. 725, f. 2. Cypselus cayanensis, Illig. Micropus cayanensis, Boie. Hirundo (Chelidon) cayanensis, Less. Pseudoprocne cayanensis, Streubel.

S. America (Cayenne, Brazil).

\section{b. Tachornis, Gosse, 1847 .}

502. Cypselus phenicobius.

B. M.

Tachornis phœnicobia, Gosse, B. of Jam. pl. Cypselus phœnicobius, G. R. $G r$.

Jamaica.

503. CYPSE LUS NIGRA.

Hirundo nigra, Gm. Briss. Orn. ii. t. 46, f. 3. Atticora nigra, Boie. Cypselus niger, G. R. Gr. Hirundo (Cecropis) nigra, Less. Gosse, B. of Jam. pl. 10. Hirundo nigra, var. B. Lath.? Pl. enl. 725, f. 1 ?

W. Indies (Jamaica, St. Domingo), S. America (Cayenne).

\section{Macropteryx.}

Macropteryx, Sw. 1832.

Macropterus, $S w$.

Pallestre, Less. 1837.

Dendrochelidon, Boie. 1838.

Hemiprocne (Chelidonia), Streubel, 1848.

504. Macropteryx Klecho.

Hirundo Klecho, Horsf. Macropteryx Klecho, G. R. Gr. M. longipennis, Sw. Zool. Illustr. n. s. pl. 74. Cypselus Klecho, Steph. C. longipennis. Temm. Pl. col. 83, f. 1. Dendrochelidon Klecho, Boie. Apus (Pallestre) Klecho, Less. Hemiprocne (Chelidonia) longipennis seu Klecho, Streubel.

Malacca, Sumatra, and Java. 
505. Macropteryx coronatus.

B. M.

Hirundo coronata, Tick. Macroptery x longipennis, Jerd. M. coronata, $\mathrm{Bl}$. Dendrochelidon velata, Less. D. schistaceus, $\operatorname{Pr} . B$.

S. and Central India and Ceylon.

506. Macroptenyx comatus.

B. $\mathrm{M}$.

Cypselus comatus, Temm. $\quad P l$. col. 268 . Macropteryx comatus, $S w$. Apus comatus et Apus (Pallestre) comatus, Less. Dendrochelidon comatus, Boie. Hemiprocne (Chelidonia) comata, Streubel.

Malacca, Sumatra, and Philippine Islands.

507. MACROPTERYX MYSTACEUS.

B. M.

Cypselus mystaceus, Less. Voy. de la Coqu.t.22. Macropteryx mystaceus, Sw. Apus mystaceus et Apus (Pallestre) mystaceus, Less. Dendrochelidon mystaceus, Boie. Hemiprocne (Chelidonia) mystacea, Streubel.

New Guinea and Amboina.

\section{Collocalia.}

Collocalia, G. R. Gr. 1840.

Salanganes, J. Geoffr.

508. Collocalia esculenta.

Hirundo esculenta, $L$. Briss. Orn. ii. t. 46, f. 2, A. Cullocalia esculenta, G. R. Gr. Hirundo (Cecropis) esculenta, Less. Hemiprocne (Salanganæ) Salangana, Streubel.

China and Borneo.

509. Collocalia nidifica.

B. M.

Hirundo esculenta, vâr. Lath. Gen. Syn. Suppl. ii. pl. 135. H. esculenta, Horsf. H. brevirostris, Mc'Clell. H. unicolor et Cypselus concolor, Jerd. Collocalia nidifica, G. R. Gr. C. brevirostris et Cotyle brevirustris, Boie.

India (Himalaya), Ceylon and Malacca. 
510? Collocalia fuciphaga.

B. M.

Hirundo fuciphaga, Thunb. Hirundo (Cecropis) fuciphaga, Less. Collocalia fuciphaga, $G . R$. Gr. C. nidifica, p. Pr. B. Hemiprocne (Salanganæ) fucivora, Streubel.

Coast of Bengal; Malayan Peninsula and Archipelago.

511. Collocalia troglodytes.

Collocalia troglodytes, G. R. Gr. \& Mitch. Gen. of B. pl, 19.

Malacca.

512. Collocalia francica.

Hirundo francica, $G m$. Collocalia francicæ, G. R. Gr.

B. $\mathrm{M}$.

Isle of France.

513 ? Collocalia delicatula.

Cypselus delicatulus, Kuhl.

\section{Acanthylis.}

a. Acanthylis, Boie, 1826.

Chætura, Steph. 1825.

Hemiprocne, p. Streubel, 1848.

Pallene, Less. 1837.

514. Acanthylis pelasgia.

B. M.

Hirundo pelasgia, $L . \quad P l$. enl. 726, f. 2 . Wils. Amer. Om. pl. 39, f. 1. Acanthylis pelasgia, G.R. Gr. Chætura pelasgia, Steph. Apus (Pallene) pelasgia, Less. Hemiprocne (Acanthỵlis) pelasgia, Streubel. Vieill. Ois. de l'Amer. Sepl. t. 33. Audub. B. of Amer. pl. 158.

N. America.

515. Acanthylis Vautil.

Cypselus Vauxii, Touns. Acanthylis Vauxi, Boie.

Columbia. 
516. Acanthylis brunneitorques. Chætura brunneitorques, Lafr. Acanthylis brunneitorques, $G, R, G r$.

S. America.

517. Acanthylis polioura.

B. M. Cypselus polioura, Temm. Pl. enl. 726, f. 2. Acanthylis polioura, $G . R$. Gi. A. brachyura, Jard.

S. America (Cayenne), and W. Indies.

518. Acanthylis Spinicauda. Cypselus spinicaudus, Temm. Pl. enl. 726, f. 1. Hirundo pelasgia, var. Lath. Acanthylis spinicauda, Boie. Hemiprocre (Acanthylis) acuta, p.? Streubel.

S. America.

\section{Acanthylis acuta.}

Hirundo acuta, Gm. Pl. enl. 544, f. 1. Acanthylis acuta, Boie. Chætura martinicana, Steph. Apus (Pallene) acuta, Less. Hemiprocne (Acantbylis) acuta, Streubel.

S. America.

520. ACANThylis oxyura.

Hirundo oxyura, Vieill. Acanthylis oxyura, Boie.

S. America (Paraguay).

521. Acanthylis Robini.

Hirundo Robini, Less. Acanthylis Robini, Boie.

Trinidad.

522. Acanthylis Sabini.

B. M. Chrtura Sabini et C. bicolor, ${ }^{\circ} \boldsymbol{G}$. Acanthylis Sabini, $G . R$. Gr. Pallene leucopygia, Boie.

W. Africa.

523. Acanthylis coracinus.

Cypselus coracinus, Miill. Acanthylis coracina, Boie. Pr. B. Consp. p. 64 .

Borneo. 
b. Hirund-apus, Hodgs. 1836.

524. Acanthylis caudacuta.

B. M.

Hirundo caudacuta, Lath. H. fusca, Steph. Gould, B. of Austr. ii. pl. 10. Chætmra australis et C. fusca, Steph. C. macroptera, Sw. Zool. Illustr.n.s. pl. 42. Pallene macroptera et P. caudacuta, Boie. Acanthylis caudacuta, G. R. Gr.

New South Wales and Van Diemen's Land.-England!

525. Acanthylis nudipes.

B. M.

Chætura (Hirundapus) nudipes, Hodgs. Acanthylis nudipes $G$. R. Gr. A. fusca, Bl. Cypselus leuconotus, Deless. Mag. de Zool. Ois. t. 20. Souven. Voy. Inde. Or. Ois. t. 9. C. nudipes, Hartl. Pallene leuconota, Boie. Hemiprocne (Acanthylis) leuconotus, Streubel.

India (Himalaya).

526. Acanthylis giǵantea.

Cypselus giganteus, $V$. Hass. $P l . c o l .364$. Apus (Pallene) gigantea, Less. Acanthylis gigantea, G.R.Gr. A. caudacuta, Bl. Pallene gigantea, Boie. Hemiprocne (Acauthylis) gigantea, Streubel.

Malayan Peninsula, India (Himalaya) and Ceylon.

527. Acanthylis leucopygialis.

Acanthylis leucopygialis, $B l$.

Malayan Peninsula.

528. Acanthylis sylvatica.

Acanthylis sylvatica et A. Sabini (Gr.), Tick.

Central India.

529. Acanthy Lis CIRIS.

Hirundo ciris, Pall. Acanthylis ciris, G. R. Gr. Pallene ciris, Boie.

Siberia. 
c. Hemiprocue, Nitzsch, 1840.

530. Acanthylis collaris.

B. M.

Hirundo collaris, Temm. Pl. col. 195. H. albicollis, Vieill. H. zonarius, Shaw? Chætura collaris, Steph. Acanthylis collaris, G.R. Gr. Hemiprocne collaris, Nitzsch. Cypselus torquatus, Licht. Apus (Pallene) collaris, Less. Pallene collaris. Boie. Hemiprocne (Acanthylis) torquata, Streubel.

S. America (Guiana, Brazil, Caraccas), and W. Indies (Jamaica, Cuba).

\section{ii. Hirundinina.}

Hirundininæ, Sw. 1831 ; Pr. B. 1838-49-50; G. R. Gr. 1840 $-41-45$.

\section{Atticora.}

Atticora, Boie, 1844.

531. Atticora fasciata.

B. M.

Hirundo fasciata, Gm. Pl. enl. 724,f.2. Sw. Zool. Illustr. n. s. pl. Atticora fasciata, Boie. Hirundo (Cecropis) fasciata, Less.

S. America (Cayenne).

532. Atricora pristoptera.

B. M.

Hirundo (Chelidon) pristoptera, Riipp. Fauna Abyss. t. 39, f.

2. Atticora albiscapulata, Boie. A. pristoptera, G. R. Gr.

Abyssinia.

533. Atticora ambrosiaca.

B. MI.

Hirundo ambrosiaca, $\mathrm{Gm}$. Pl. col.460, f. 2. Atticora ambrosiaca, Boie. Apus parvus, Less. Hirundo (Cecropis) ambrosiaca, Less. Cypselus parrus, Licht. C. ambrosiacus, G. R. Gr. Dendrochelidon ambrosiacus, $\operatorname{Pr} . B$.

W. ; N.-E. and E. Africa. 
534. Atricora velox.

Hirundo velox, Vieill. Levaill. Ois. de l'Afr. t. 244, f. 2. Apus velox, Less. Atticora velox, $\operatorname{Pr} . B$.

S. Africa.

535. Atticora cristata.

Hirundo cristata, Steph. Levaill. Ois, de l'Afr.t. 247. Hirundo (Cecropis) cristata, Less. Dendrochelidon cristatus, Boie.

S. Africa.

536. Atticora leucosternon.

B. $\mathrm{M}$.

Hirundo leucosternus et Atticora leucosternon, Gould, $B$. of Austr. ii. pl. 12. Chelidon leucosterna, Boie.

New South Wales; S. and W. Australia.

537. Atticora vanikorensis.

Hirundo vanikorensis, Quoy \& Gaim. Voy. de l'Astrol. Ois. t. 12, f. 3. Atticora? vanikorensis, G. R. Gr. Cotyle vanikoreusis, Boie. Hirundo (Herse) vanikorensis, Less.

New Guinea.

Examine Macropteryx spodiopygius, Peale, Expl. Exped. viii. p. 176, pl. 49, f. 2 ,

Samoan and Feejee Islands.

\section{Hirundo.}

a. Hirundo, L. 1735 .

Cecropis, Boie, 1826.

Herse, Less. 1837.

538. Hirundo rustica.

B. M.

Hirundo rustica, $L . \quad P l$. enl. 543, f. 1. Gould, B. of Eur. pl. 54. Yarr. Brit. B. 2 edit. ii. fig. p. 231. H. domestica, Pall. H. albifrons, Rüpp. Cecropis rustica, Boie, Brehm. C. pagorum, Brehm. Hirundo (Cecropis) rustica, Less.

Great Britain ; Europe. W.; N. and N.-E. Africa. 
539. Hirundo cahirica.

Hirundo cahirica, Licht. H. castanea, Sav. Hist. de l'Egypte. Ois. t. 4, f. 4. H. Riocouri, Sav. H. Savignyi, Leach. H. Boissoneauii, Temm. H. rustica orientalis, Schl. Cecropis Savignyi, Boie. Hirundo (Cecropis) Savignyi, Less.

S. Europe; W. ; N. and N.-E. Africa.

540. Hirundo Smithit.

Hirundo Smithii, Leach. Cecropis Smithii, Boie.

W. Africa (Congo).

541. Hirundo Rutila.

Hirundo rutila, Vieill. Cecropis rutila, Boie.

542. Hirundo gutturalis.

B. M.

Hirundo gutturalis, Scop. Sonn. Voy. t. 76. H. panayana, Gm. H. jewan, Sykes. H. rustica, Bl. Meyen, Nova Acta, 1834, t. 10, f. 1, Suppl. H. javanica, Sparr. Mus. Carls. t. 100. Pl. col. 83, f. 2. H. rusticoïdes, Kuhl. Cecropis panayana, C. jewan, et C. javanica, Boie. Hirundo (Herse) javanica. H. (Cecropis) panayana et H. (Herse) jewan, Less.

India, China, Java, Japan and Philippine Islands.

543. Hirundo domicola.

Hirundo domicola, Jerd. H. rustica, Lath.

Malacca and Nilgiris.

544. Hirundo erontalis.

B. M.

Hirundo javanica, Vig. \& Horsf. H. frontalis, Quoy \& Gaim.

Voy. de l'Astrol. Ois. t. 12, f. 1. H. neoxena, Gould, B. of Austr. ii. pl. 13. H. pacifica, Griff. An. Kingd. ii. pl. p. 95. Cecropis frontalis, Boie. Hirundo (Herse) frontalis, Less.

Australia (except the North).

545. Hirundo RUfifrons.

Hirundo rufifrons, Shaw. Levaill. Ois. de l Afr. t. 245, f. 2.

Cecropis rufifrons, Boie. Hirundo (Cecropis) rufifrons, Less.

S. and W. Africa. 
546. Hirundo albigularis.

Hirundo albigularis, Strickl. Contr. Ornith. 1849, pl. 17. H. rufifrons, Less. H. albigula, $\operatorname{Pr} . B$.

S. Africa.

547. Hirundo daurica.

B. M

Hirundo daurica, $L$. H. alpestris, Pall. Zoogr. t.30, f. 2. H. erythropygia, Sykes. H. nipalensis, Hodgs. H. europygia, Tick.? Cecropis daurica et C. erythropygia, Boie. Hirundo (Cecropis) daurica et H. (C) erythropygia, Less.

Siberia, India and China.

548? Hirundo Japonica.

Hirundo alpestris japonica, Temm. \& Schl. Fauna Jap. t. 11. H. japonica, $\operatorname{Pr} . \ddot{B}$.

Japan.

549. Hirundo hyperythra.

Hirundo hyperythra et Herse hyperythra, $B l$.

Ceylon.

550. Hirundo striolata.

Hirundo striolata, Temm. \& Schl. Cecropis striolata, Boie. Java.

551. Hirundo abyssinica.

B. M. Hirundo striolata et H. capensis, Rüpp. Syst. Uebers. Vög. von N. Ost. Afr. t. 6 . H. abyssinica, Guér.

N.-E. and W. Africa.

552. Hirundo Korthalsi.

Hirundo Korthalsi, Pr. B. Consp. p. 340.

553. Hirundo cucullata.

B. $\mathrm{M}$.

Hirundo cucullata, Bodd. Pl. enl. 723, f. 2. H. capensis, Gm. Cecropis capensis, Boie. Hirundo (Cecropis) capensis, Less.

S. Africa. 
554. HirUNDO RUFUla.

B. M. Hirundo rufula, Temm. Levaill. Ois. de l'Afr. t. 245, f. 1. H. daurica, Savi. H. alpestris, $\operatorname{Pr}$. B. H. melanocrissus, G. R. Gr. Cecropis melanocrissus, Rüpp. Syst. Uebers. Vög. N. Ost. Afr. t. 5. C. rufula, Boie.

Africa and S. Europe.

555. Hirundo SENEgalensis.

B. M.

Hirundo senegalensis, $L . \quad P l$. enl. $310 . \quad S w . B$. of $W . A f r$. pl. 6. H. rufula, Gould, B. of Eur. ii. pl. 55. Hirundo (Cecropis) senegalensis, Less. Cecropis senegalensis, Boie.

W. and N.-E. Africa.

556. Hirundo nigricans.

B. M.

Hirundo nigricans, Vieill. H. pyrrhonota, Lath. MSS. Vig. \&. Horsf. Voy. de l'Astrol. Ois. t. 12, f. 2. "H. pygialis, Temm." Pr. B. Cecropis nigricans et C. pyrrhonota, Boie. Collocalia arborea, Gould, B. of Austr. ii. pl. 14. Chelidon arborea, Gould. Hirundo (Herse) nigricans et H. (H) pyrrhonota, Less. Herse nigricans, Pr. $B$.

Australia.

557. HirUNDO ARIEL.

B. M.

Collocalia ariel, Gould, B. of Austr. ii. pl. 15. Chelidon ariel, Gould. Hirundo ariel, G. R. Gr. Herse ariel, Pr. B.

New South Wales; S. and W. Australia.

558. Hirundo tahitica.

Hirundo tahitica, Gm. Lath. Gen. Syn. pl. in titlepage. H. pyrrhomæla, Forst. H. taitensis, Less. Hirundo (Herse) taitensis, Less. Cecropis taitensis, Boie. Herse tahitica, $P r$. $B$.

Society Islands (Otaheite).

559. Hirundo puella.

Hirundo puella, Schl. Fauna Jap. p. 33.

W. Africa (Gold Coast). 
560. Hirundo leucosoma.

Hirundo leucosoma, Sw. Chelidon leucosoma, Boie.

B. M.

W. Africa.

561. HirUn Do Nigrita.

B. $\mathrm{M}$.

Hirundo nigrita, G. R. Gr. Mitch. Gen. of B. pl. 20. Atticora nigrita. $\mathrm{Pr}$. $\mathrm{B}$.

W. Africa.

562. Hirundo filifera.

B. M.

Hirundo? filifera, Steph. Lath. Hist. of B. pl. 113. H. filicaudata, Frankl. H. ruficeps, Licht. Chelidon ruficeps, Boie. Hirundo (Cecropis) filifera, Less.

India and Abyssinia.

563. Hirundo ertth rocephala.

B. M.

Hirundo ery throcephala et H. indica? Gm. Lath. Gen. Syn. iv. pl. 56. Hirundo (Cecropis) indica, Less. Chelidon erythrocephala et C. indica, Boie. Herse erythrocephala, Pr. B. India.

564. Hirundo Wilsoni.

B. M.

Hirundo americana, Wils. Amer. Orn. pl. 38, f. 1, 2. H. rufa, Vieill. Ois, de l'Amer. Septr. t. 30. H. rustica, Audub. B. of Amer. pl. 173.

N. America.

565. Hirundo erythrogaster.

Hirundo erythrogaster, Bodd. Pl. enl. 724 , f. 1 . H. rufa, $G m$. Cecropis rufa, Boie. Hirundo (Cecropis) rufa, Less.

S. America (Cayenne).

566. Hirundo americana.

Hirundo americana, Gm. H. platensis, Steph. Cecropis americana, Boie.

S. America.

Examine, Hirundo ruficollaris, Peale, U. S. Expl. Exped. viii. p. 175 , pl. 49 , f. 1.

S. America (Peru). 
567. Hirundo Fulva.

B. M.

Hirundo fulva, Vieill. Ois. de l'Amer. Sept. t. 32 . H. pæciloma, Gosse. H. respublicana, Clyton. Cecropis fulva, Boie, Hirundo (Cecropis) fulva, Less.

W. Indies (Jamaica).

568. Hiru do melanogaster.

Hirundo melanogaster, $S w$. Cecropis fulva, p. Boie. Hirundo (Biblis) melanogaster, Less.

Central America (Mexico).

569. Hirundo Lunifrons.

Hirundo lunifrons, Say. Pr. B. Amer. Orn. pl. 7, f. 1. Audub. B. of Amer. pl.68. Cecropis lunifrons, Boie.

N. America (Rocky Mountains).

570. Hirundo thalassina.

B. $\mathbf{M}$.

Hirundo thalassina, Sw. Audub. B. of Amer. pl. 385, f. 4, 5 . H. viridis, Licht.? Chelidon thalassina, Boie. Hirundo (Cecropis) thalassina, Less.

Central America (Mexico).

57l. Hirundo Euchrysea.

B. M.

Hirundo euchrysea, Gosse, B. of Jam. pl. 12.

W. Indies (Jamaica).

572. Hirundo bicolor.

B. M.

Hirundo bicolor, Vieill. Ois. de l'Amer. Sept. t. 31 . H. viridis, Wils. Amer. Orn. pl. 38, f. 3. H. leucogaster, Steph. Hirundo (Chelidon) bicolor, Less. Chelidon viridis et Chelidon leucogastra, Boie. Hirundo (Chelidon) bicolor, Less. Audub. B. of Amer. pl. 98.

N. America and Mexico.

573. Hirundo albiventer.

B. $M$. Hirundo albiventer, Bodd. Pl. enl. 546, f. 2. H. leucoptera, $\mathrm{Gm}$. Chelidon leucoptera, Boie. Hirundo (Chelidon) leucoptera, Less.

S. America (Para, Demerara, Bolivia, Peru). 
574. Hirundo maculata.

Hirundo maculata, Bodd. $\quad P l$. enl. 546. f. 1. H. leucoptera S. America.

var. $\beta$., Lath. H. maculosa, Kuhl. Chelidon maculata, Boie.

575. Hirundo melanoleuca.

Hirundo melanoleuca, Pr. Max. Pl.col. 209, f. 2. Chelidon melanoleuca, Boie. Hirundo (Cecropis) melanoleuca, Less.

S. America (Brazil, Chili).

576. Hirundo cyanoledca.

Hirundo cyanoleuca, Vieill. $P l$. pyga, Licht. H. minuta, Pr. noleuca, et H. (Herse) minutax. Hirundo (Chelidon) cyaBoie.

S. America (Brazil, Para, Bolivia, La Planta, Argentina, Peru).

577. Hiruñdó LeUCORRHó.

Hirundo leueorrhöa, Vieill. H. leucopyga, Licht. Nova Acta, 1834, pl. 10, Suppl. Chelidon leucorrhoa, Boie.

S. America (Chili, Paraguay).

578. Hirundo Frontalis.

Hirundo frontalis, Gould. Chelidon fruntata, Boie.

S. America.

579. Hirundo patagonica. Hirundo patagonica, D'Orb.\& Lafr. Chelidon patagonica,
Boie.

S. America (Patagonia).

580. Hirundo andicola.

Hirundo andicola, D'Orb. \& Lafr. Chelidon andicola, Boie.

S. America (Bolivia, Peru).

581. Hirundo cinerea.

Hirundo cinerea, Gm. Furst. Icon. ined. 168. Cecropis??
cinerea, Boie.

S. America (Peru) and Society Islands. 
Examine, Macropteryx leucophæus, Peale, U. S. Expl. Exped. viii. p. 178, pl. 49, f. 3.

Island of Tahiti.

582. Hirundo peruviana.

Hirundo peruviana, Gm. Cecropis?? peruviana, Boie.

S. America (Peru).

583. Hirundo cyanopyraha.

Hirundo cyanopyrrha, Vieill. Cecropis cyanopyrrha, Boie.

S. America (Brazil).

584. Hirundo unalaschiensis.

Hirundo unalaschkensis, Gm.

Chelidon unalaschkensis, Boie.

H. aoonalaschkensis, Lath.

Kamtchatka.

c. Cotyle, Boie, 1822 .

Biblis, Less. 1837.

? Ptyonoprogne, Reichenb. 1850.

585. Hirundo riparia.

B. M.

Hirundo riparia, $L . \quad P l$. enl. 543, f. 2. Gould, B. of Eur. pl. 58. Yarr. Brit. B. 2 edit. ii. fig. p. 251 . H. cinerea, Vieill. Cotyle riparia, Boie, Brehm. C. fluviatilis et C. microrhynchos, Brehm. Hirundo (Cotyle) riparia, Keys. \& Bl.

Great Britain; Europe, N. \& Central India, Persia, N. \& N.-E. Africa.

586. Hirundo palustris.

Hirundo palustris, Steph. Levaill. Ois. de l'Afr. t. 246, f. 2. H. paludicola, Vieill. Cotyle palustris, Boie. C. paludibula, Riipp.

N.-E. and S. Africa.

587. Hirundo Rupestris.

B. II.

Hirundo rupestris et H. montana, Gm. Gould, B. of Eur. pl. 56. H. rupicola, Hodgs. H. inornata, Jerd. Cotyle rupestris et Chelidon rupestris, Boie. Hirundo (Biblis) rupestris, Less.

Europe, India (Himalaya, Nilgiris), and Egypt. 
588. Hirundo fuligula.

B. M.

Hirundo fuligula, Licht, Levaill. Ois. de l'Afr.t. 246, f. 1. H. rupestris, juv. Licht. H. torquata? Steph. Cotyle fuligula, G. R. Gr. Hirundo (Cecropis) torquata, Less. Cecropis torquata, Boie.

S. Africa.

589. Hirundo cincta.

B. M.

Hirundo cincta, Bodd. Pl. enl. 723, f. 1. H. torquata, Gm. Cotyle cincta, G. R. Gr. C. torquata, Boie.

N.-E. and S. Africa.

590. Hirundo sinensis.

B. M.

Hirundo sinensis, Gr. Ill. Ind. Zool. pl. 35, f. 3. H. brevicaudata, Mac'Clell. \& Horsf. H. subsoccata et H. minuta, Hodgs. Cotyle brevicaudata, Boie. C. sinensis, G. R. Gr.

India, Burmah and China?

591. Hirundo concolor.

B. M. Hirundo concolor, Sykes. Cotyle concolor, Boie. Hirundo (Biblis) concolor, Less.

Central India.

592. Hirundo Borbonica.

Hirundo borbonica, Gm. Cotyle borbonica, Boie.

Isle of France.

593. Hirundo virescens.

Hirundo horbonica, var. Gm. Pl. enl. 544, f. 2. H. virescens, Vieill. Cotyle, sp. 4, Boie.

Isle of France.

594. Hirundo SERripenNis.

Hirundo riparia, Wils. Amer. Om. pl. 38, f. 4. H. serripennis, Audub. B. of Amer. pl. 385, f. 1, 2,3. Cotyle serripennis, Boie.

N. America. 
595. Hirundo tapera.

Hirundo tapera, L. Briss. Orn. ii. t. 45, f. 3. Hirundo (Cecropis) tapera, Less. Cotyle tapera, Boie. Hemiprocne (Acauthylis) acuta, p. ?? Streubel.

S. America (Cayenne, Brazil), and W. Indies, (Jamaica).

596. Hirundo fucata.

B. M.

Hirundo fucata, Temm. Pl. col. 161, f. 1. H. pyrrhonota, Vieill. Cotyle fucata et Cecropis pyrrhonota, Boie. Hirundo (Herse) fucata, Less.

S. America (Brazil).

597. Hirundo Flavigastra.

B. $M$.

Hirundo flarigastra, Vieill. $P l . c o l .161$, f. 2 . H. jugularis, Pr. Max. H. hortensis, Frey. Cotyle flavigastra, Boie. C. jugularis, G. R. Gr. Hirundo (Herse) jugulatis, Less.

S. Anerica (Bahia, Caraccas, Brazil, Argentina).

598. ? HiRUNDO RUFICOLLIS.

H. ruficollis, Vieill. Cotyle? ruficollis, G. R. Gr. Cecropis ruficollis, Boie.

S. America (Brazil).

d. Chelidon, Boie, 1822.

599. Hirundo URBica.

Hirundo urbica, $L$. $P l$ enl $542, f$. H. domestica, Leach. Chelidon urhica, H. lagopoda, Pall. nestrarum et C. rupestris, Brehm. Hirundo (Chehm. C. feKeys. \&. Bl. Gould, B. of Eur. pl. 57. Yarr. Brit. B.2 edit. ii. fig. p. 245.

Great Britain ; Europe, India (Nilgiris), N. \& N.-E. Africa.

600. Hirundo dasyeus.

Hirundo dasypus, Temm. Chelidon dasypus, $\operatorname{Pr}$. B. Consp. p. 343.

Borneo. 


\section{Progne. \\ Progne, Boie, 1826.}

601. Progne purpurea.

B. M.

Hirundo purpurea et H. subis, L. Pl. enl. 722. Edw.B. pl. 120. H. violacea, Gm. H. cærulea, Vieill. Ois. de l'Amer. Sept. t. 26, 27. H. versicolor, Vieill. H. ludoviciana, Cuv. Progne purpurea, Boie. Hirundo (Cecropis) violacea, Less. H. (C) subis, Less. Wils. Amer. Orn. pl. 39, f. 1, 2. Yarr. Brit. B. 2 edit. ii. fig. p. 257.

Great Britain? N. America and S. America (Bahia, Bolivia, La Plata, Patagonia, Peru).

602. Progne domestrca.

B. M. Hirundo domestica, Vieill. Progne chalybea, p. Boie. Progne domestica, G. $R$. Gr.

S. America (Bolivia).

603. Progne modesta.

B. $M$. Hirundo conculor, Gould. Progne modesta, Gould, Voy. of Beagle, pl. 5.

S. America (Chili).

604. Progne dominicensis.

B. 11 . Hirundo dominicensis, $\mathrm{Gm}$. $P$ l. enl. 545, f. $1 \mathrm{H}$. albiventris, Vieill. Ois. de l'Amer. Sept. t. 28, 29. Progne dominicensis, Boie.

S. America, (Para); Central America and W. Indies (St. Domingo, Jamaica).

605. Progne chalybea.

B. M. Hirundo chalybea, Gm. Pl. enl. 545, f. 2. Briss. Orn. ii, t. 46, f. 1. Progne chalybea et Cecropis chalybea, Boie. Hirundo (Cecropis) chalybea, Less.

S. America (Para, Cayenne).

606. Progne fusca.

B. M.

Hirundo fusca, Vieill. Progne fusca, G. R. Gr.

South America (Brazil, La Plata, Bolivia). 
607. Progne pascuum.

Hirundo pascuum, Pr. Max. Progne pascuum, Boie.

S. America (Brazil).

\section{CORACIAD五.}

Coraciadx, G. R. Gr. 1845 ; Cab. 1847 ; Pr. B. 1849-50.

Todidæ, Vig. 1825; Sw. $1831 ;$ G. R. Gr. 1840-41; Selys, p. 1842 ; Pr. B. $1849-50$.

Meropidæ, p. $S w .1837$.

Curvidæ, p. Vig. 1825.

Ampelidæ, p. Pr. B. 1831-38.

Eurylaimidæ, Selys, $1842 ; \operatorname{Pr} . B .1850$.

Momotidæ, Pr. Mux.; Selys, 1842; Bl. 1849.

Prionitidæ, Pr. B. $1849-50$.

Buceridæ, p. Vig. 1825; Pr. B. 1831.

Coracidæ et Pipridæ, p. $B l .1849$.

Platyrhynchides, p. et Curaciades, Sundev. 1835.

i. Coraciante.

Coracianæ, G. R. Gr. 1840-41-45; Cab. 1847; Pr. B. 184950.

Coraciana, Vig. 1825.

Corvinæ, p. et Ampelinæ, p. Pr. B. 1831.

\section{Brachypteracias.}

a. Brachypteracias, Lafr. 1834.

Chloropygia, Sw. 1837.

608. Brachypteracias leptosomus.

Colaris leptosomus, Less. Illustr. de Zool. t. 20. Brachyptera. cias leptosomus, Lafr. Mag. de Zool. 1844, Ois. t. 31. Chloropygia leptosomus, $S w$.

Madagascar.

b. Atelornis, Lafr. 1846.

609. Brachypteracias Pitroldes.

B. $M$.

Brachypteracias pittuides, Lafr. Mag. de Zool. 1834, t. 32.

Atelornis pittoildes, Pucher.

Madagasear. 
610. Brachypteractas squamigera.

Brachypteracias squamigera, Lafr. $O$. des Murs, Icongr. Ornith.t. 39. Atelornis squamigera, Pucher.

Madagascar.

\section{Coracias.}

Coracias, L. 1735.

Galgulus, $\mathrm{Br} .1760$.

611. Coracias garrula.

B. M.

Coracias garrula, $L . \quad P l . e n l .486 . \quad$ C. germanicus et C. planiceps, Brehm. Galgulus garrulus, Vieill. Levaill. Ois. de Parad. t. 32, 33. Gould, B. of Eur. pl. 60. Yarr. Brit. B. 2 ed. ii. fig. p. 211.

Great Britain ; Europe, India (Moultan, Afghanistan) and N. and W. Africa.

612. Coracias Indica.

B. M.

Coracias indica et C. bengalensis, $L$. Pl. enl. 285. Edw. B. pl. 326. C. nævia, Wagl. C. affinis, Mc'Clell. G. R. Gr.\& Mitch. Gen. of B. pl. 21. Garrulus (Galgulus) nævius et Galgulus nævius, Vieill. Levaill. Ois. de Parad. t. 27.

India (Assam, Birmab, Tanesserim).

613. Coracias pilosa.

B. M.

Coracias pilosa, Lath. Levaill. Ois. de Parad. t. 28, $29 . \quad$ C. crinita, Shaw. C. nuchalis, Sw. C. Levaillantii, Rüpp. Coracias (Galgulus) pilosa et Galgulus pilosus, Vieill.

N. and W. Africa.

614. Coracias caudata.

B. M.

Coracias caudata, L. $P l . e n l .88,626$. C. abyssinica et C. senegalensis, $G m$. C. senegala, Lath. C. albifrons et C. angolensis, Shaw. C. natalensis, Licht. Galgulus caudatus, Vieill. Levaill. Ois. de Parad. t. 25. Edw. B. pl.327. O. des Murs, Icongr. Ornith. t. 28.

N. and W. Africa. 
615. Coracias cyanogaster.

B. $M$.

Coracias cyanogaster, Cuv. Levaill. Ois. de Parad. t. 26. Garrulus (Galgulus) cyanogaster et Galgulus cyanogaster, Vieill. Jard.\&Selby, Illustr. Orn. pl. 123. Sw. B. of W. Afr. pl. 13.

W. Africa.

616. Coracias Temminckir.

B. $M$.

Coracias Temminckii, Wagl. Levaill. Ois. de Parad. t. 6. C. papuensis et C. D'Urvillei, Quoy et Gaim. Voy. de l'Astrol. Ois. t. 16. C. pileata, Reinu. Garrulus (Galgulus) Temminckii et Galgulus Temminckii, Vieill.

Celebes and Moluccas.

\section{Sigmodus.}

Sigmodus, Temm. 1850.

617. Sigmodus caniceps.

Sigmodus caniceps, Temm. Pr. B. Consp. p. 365.

B. M.

W. Africa (Ashantee).

\section{Eurystomus.}

Eurystomus, Vieill. 1816.

Colaris, Cuv. 1817.

618. Eurystomus orientalis.

B. M.

Coracias orientalis, $L . \quad P l . e n l .619 . \quad$ Eurystomus orientalis, Steph. E. fuscicapillus et E. cyanicollis, Vieill. E. calornyx, Hodgs. Colaris orientalis, Cuv. C. cyanicollis, Wagl. Levaill. Ois, de Parad. t. 36. Gal. des Ois. t. 111.

India, China (?), Malacca and Ceylon.

619. EURystomus pacificus.

B. M.

Coracias pacifica, Lath. Eurystomus pacificus, G.R. Gr. E. orientalis, Vig. \& Horsf. E. australis, Sw. Gould, B. of Austr. ii. pl. 17. Coracias (Galgulıs) pacifica et Galgulus pacific us, Vieill.

New South Wales and N. Australia. 
620. Eurystomus AFER.

B. M.

Coracias afra, Lath. Shaw, Nat. Misc. pl. 401. Eurystomus purpurascens et E. rubescens, Vieill. E. afer, Steph. Levaill. Ois. de Parad. t. 35. Colaris afra, Cuv. C. purpurascens, Wagl.

Africa.

621. Eurystomus gularis.

B. M.

Eurystomus gularis, Vieill. Levaill.Ois. de Parad.t. 56. E. collaris, Vigors. Colaris gularis, Wagl.

New Guinea.

622. Eurystomus madagascariensis.

Coracias madagascariensis, $\mathrm{Gm} . \mathrm{Pl}$. enl. 501. Eurystomus madagascariensis, G. R. Gr. E. violaceus, Vieill. Colaris violaceus, Wagl. Levaill. Ois. de Parad. t. 34.

Madagascar.

623. Eurystomus viridis.

Coracias viridis, Cuv. Levaill. Ois. de Parad. t. 31. C. cyanea, Gm.? C. vivida, Lath.? Colaris viridis, Wagl. Eurystomus viridis, Hartl. Galgulus viridis, Vieill. Gal. des Ois. t. 110.

Africa.

ii. Eurylaimine.

Eurylaiminæ, Sw. 1837; G. R. Gr. 1840-41-47; Cab. 1847 ; Pr. B. $1849-50 ; B l .1849$.

\section{Eurylaimus.}

a. Eurylaimus, Horsf. 1820. Platyrhynchus, Vieill. 1825.

624. Eurylaimus javanicus. B. M. Eurylaimus javanicus, Horsf. Zool. Res. pl. E. Horsfieldii, Termm. Pl. col. 130, 131. Platyrhynchus Horsfieldi, Vieill. Gal.des Ois. t. 125. Sw. Nat. Libr. Flyc. pl. 30.

Malayan Peninsula and Archipelago. 
625. Eurylatmus ochromalus.

B. M.

Eurylaimus ochromalus, Raffl. E. cucullatus, Temm. $P l$. col.261. E. Rafflesii, Less.

Malayan Peninsula and Archipelago.

b. Serilophus, $S w .1837$.

Sericolophus, Reichenb. 1850.

626. Eurylaimus lunatus.

B. M.

Eurylaimus lunatus, Gould, $T r$. Zool. Soc, i. pl. 25. Serilophus lunulatus, $S w$.

Malayan Peninsula and Archipelago.

627. Eury laimus rubropygius.

B. M.

Raya rubropygia. Simornis rubropygia et Serilophus rubropygitus, Hodgs. Eurylaimus rubropygius, G. R. Gr. \& Mitch. Gen. of B. pl. 23. E. lunatus, Mc Clell.

India (Himalayah Mountains).

c. Psarisomus, $S w .1837$.

Crossodera, Gould, 1837.

Raya, 1839; Simus, 1841 et Simornis, 1844, Hodgs.

628. Eurylaimus Dalhousiar.

B. M.

Eurylaimus Dalhousiæ, James. E. psittacinus, Müll. Pl. col. 598. Raya seriogula et R. nipalensis, Hodgs. Simornis (Raya) seriogula, Hodgs. Psarisomus Dalhousix, Su. Crossodera Dalhousiæ, Gould, Icon. Av. pl. Royle, Illustr. of Bot. pl. 7, f. 2.

India (Himalayah Mountains).

d. Corydon, Less.

629. Eurylaimus sumatranus.

B. M.

Coracias sumatranus, Raff. Eurylaimus sumatranus, Vig.

E. corydon, Temm. Pl. col. 297. Corydon Temmincki, Less.

C. sumatranus, Strickl.

Malayan Peninsula and Archipelago. 


\section{Peltops.}

Peltops, Wagl. 1829.

Erolla, Less. 1831.

Platystomus, $S w .1837$.

6:30. Peltops Blainvillit.

Eurylaimus Blainvillii, Garn. Voy. de la Coqu. t. 19, f. 2. Nat. Libr. x. Flycatch. pl. 31. Erolla Blainvillii, Less. Platystomus Blainvillii, Sw. Peltops Blainvillii, Wagl.

New Guinea.

\section{Cymbirhynchus.}

Cymbirhynchus, Vigors, 1831.

631. CyMBIRHYNCHUS MACRORHYNCHUS.

B. M.

Todus macrorhynchos, Gm. T. nasutus, Lath. Gen. Syn. pl. 30. Platyrhynchus nasutus, Desm. Eurylaimus nasutus, Tem. Pl. col. 154. E. lemniscatus, Raff. Cymbirhynchus nasutus, Vig. C. macrorhyuchus, G. R. Gr. C. affinis, $B l$. Erolla nasica, Less.

Malayan Peninsula and Archipelago.

\section{iii. Todinte.}

Todinæ, G. R. Gr. $1840-41-47$; Pr. B. 1849-50.

Muscicapinæ, p. $\operatorname{Pr} . B .1831 ; S w .1837$.

\section{Todus.}

Todus, L., 1766.

632. TODUS VIRIDIS.

Todus viridis, L Vieill. Ois de l'Amer. Sept. 56 B. M. Zool. Illustr. n. s. pl. 96.

W. Indies (Jamaica). 
633. Todus Multicolor.

B. M.

Todus multicolor, Gould, Icon. Av. pl. T. portoriensis, Less. T. viridis, Vig. Voy. de l'Isle de Cuba, Ois. t. 22.

Cuba.

634. Todus mexicanus.

B. $\mathrm{M}$.

Todus mexicanus, Less. T, viridis, Vieill. Atl. de Dict. Sci. Nat. t. 32 , f. 1.

Central America.

635. Todus subulatus.

Todus subulatus, Gould. T. dominicensis, Lafr. Vieill. Gal. des Ois. t. 124?' Pl. enl. 585, f. 1, 2?

St. Domingo.

iv. Momotinæ.

Momotinæ, G. R. Gr. 1840-41-47.

Prionitinæ, Cab. 1847 ; Pr. B. 1849-50.

\section{Momotus.}

a. Momotus, Briss. 1760.

Baryphonus, Vieill. 1816.

Prionites, rll. 1811.

636. Momotus Brasiliensis.

B. II.

Momotus brasiliensis, Lath. Edw. B. pl. 370. Levaill. Prom, t. 37, 38. Ramphastos momota, L. Pl. enl. 370. Baryphunus cyanocephalus, Vieill. Prionites mormota, Illig.

S. America (Brazil, Cayenne, Peru).

637. Momotus bahamensis.

B. M.

Prionites bahamensis, Sw. Jard. \& Selby, Illustr. Orn. n. s. pl. 45. Momotus bahamensis, G. R. Gr.

Bahama Islands, Trinidad and Tobago. 
638. Momotus cerruleiceps. B. M. Prionites cæruleiceps, Gould. P. cæruleocephalus, Jard. \& Selby, Illustr. Orn. n. s. pl. 42. Momotus cæruleiceps, G. R. Gr.

Central America (Mexico).

639. MомотUS RuFicapillus.

Prionites ruficapillus, Illig. Levaill. Roll. t. 39. P. Dombeyanus, Ranz. Momotus rubricapillus, Steph. M. ruficapillus, G. R. Gr. M. Dombeyi, Less. Baryphonus cyanogaster, Vieill.

S. America (Brazil, Peru).

640. Momotus Levaillantit.

B. M.

Momotus Levaillantii, Less. Levaill. Hist. Nat. Prom. Suppl. t. B. Baryphonus ruficapillus, Vieill. Gal. des Ois. t. 190. Prionites Levaillantii, $P r . B$.

S. America.

641. Momotus mexicanus.

B. M.

Momotus mexicanus, Sw. Zool. Illustr. n. s. pl. 81. M. Martii, Jard.\& Selby, Illustr. Orn. pl. 23. Prionites mexicanus, $\operatorname{Pr} . B$.

Central America (Mexico).

642. Momotus yucatensis.

Momotus yucatensis, Cabot. Prionites yucatanensis, $P r, B$. Central America.

643. Momotus GUlaRis.

Prionites gularis, Lafr. Momotus gularis, G. R. Gr.

Central America (Guatimala).

644. Моmotus Lessonil.

Momotus Lessonii, Less. O. Des Murs, Icongr. Ornith. pl.

Prionites Lessonii, $P r . B$.

Central America (Guatimala). 
b. Hylomanes, Licht. 1838.

645. Momotus momotula.

B. M.

Hylomanes momotula, Licht. Abhandl. Akad. zu Berl. 1838, p. 444, t. 4. Momotus momotula, G. R. Gr.

Central America (Mexico).

c. Crypticus, $S w .1837$.

646. Momotus superciliaris.

B. M.

Prionites (Crypticus) superciliaris, Sandb. Jard. \& Selby, Illustr. Orn. n. s. pl. 18. Crypticus superciliosus, Sw. C'. apiaster, Less. Momotus superciliaris, G. R. Gr.

Central America (Honduras).

647. Momotes Martit.

Prionites Martii, Spix, Av. Bras. t. 60. Momotus platyrhynchus, Leadb. Jard. \& Selby, Illustr. of Orn. pl. 106. Crypticus platyrhynchus, G. R. Gr. C. Martii, $P r . B$.

S. America (Brazil, Perı, Columbia).

648. Momotes carinatus.

Prionites carinatus, DuBus. Crypticus carinatus, Lufr. Momotus carinatus, G.R. $G r$.

Central America (Guatimala).

\section{TROGONIDE.}

Trogonidæ, Sw. 1831-37; G. R. Gr. 1840-41-45; Cab. 1847; Pr. B. $1849-1850 ; B l .1849$.

Cuculidæ, p. Vig. 1825 ; p. Pr. B. 1831.

Trogonides, Sundev. 1835.

\section{i. Trogonine.}

Trogoninæ, Pr. B. 1849-50.

Bucconinæ, p. Pr. B. 1831 . 


\section{Trogon.}

a. Trogon, L. 1766; Mcehr, 1752.

649. Trogon mexicanus.

B. M.

Trogon mexicanus, Sw. Zool. Illustr.n. s. pl. 82, 83. T. glocitans, Licht. Gould, Monogr. of Trog. pl. 1,2. T. capistratus, Less.?

Central America (Mexico).

650. Trogon elegans.

Trogon elegans, Gould, Monogr. of Trog. pl. 3.

B. M.

Central America (Guatimala, Mexico).

651. Trogon ambiguus.

Trogon ambiguus, Gould, Monogr. of Trog. pl. 4.

Central America (Mexico).

652. Trogon XaLAPENSIS.

Trogon xalapensis, DuBus, Esquis. Ornith. t. 2. T. auratus, Lafr.

Central America (Mexico).

653. Trogon collaris.

B. M.

Trogon collaris, Vieill. Levaill. Hist. Nat. Courc. t. 6. T. rosalba, Cuv. T. anratus, $S w . \quad$ Gould, Monogr. of Trog. pl. 5. T. castaneus, Spix, Av. Bras. t. 37.

S. America (Cayenne, Brazil, Guiana, Venezuela).

654. Trogon PUElLA.

Trogon puella, Gould.

Central America (Escuintla).

655. Trogon Variegatus.

B. M.

Trogon variegatus, Spix, Av. Bras. t. 37. Gould, Monogr. Trog. pl. 6.

S. America (Brazil, Paraguay). 
656. Trogon purpuratus.

Trogon purpuratus, Sw. B. of Braz. pl. 63, 64 .

S. America (Brazil).

657. Trogon LeUCURUS.

Trogon leucurus, $S w$.

S. America (Brazil).

658. Trogon caligatus.

Trogon caligatus, Gould, Monogr. of Trog. pl. 7.

B. M.

S. America (Venezuela? Guiana?).

659. Trogon atricoltis.

B. M.

Trogon rufus, $\mathrm{Gm} . \quad \mathrm{Pl}$. enl. 736. T. lepturus, Sw. Pl. enl. 195. T. atricollis, Vieill. Gould, Monogr. of Trog. pl. 8. Levaill. Hist. Nat. Courc. t. 7, 8, 9, 15? Lath. Gen. Syn. pl. 21. T. scalaris, Licht.

S. America (Guiana, Cayenne).

660. Trogon personatus.

Trogon personatus, Gould.

Cordillerian Andes.

661. Trogon sulphureus. Trogon sulphureus, Spix, Av. Bras. t. 38. T. meridionalis, Sw.P Gould, Monogr. of Trog. pl. 9?

Trinidad; S. America (Cayenne, Brazil), and Bahama Islands.

662. Trogon viridis.

B. M.

Trogon viridis, $L . \quad P l$. enl. $765,766 . \quad$ T. violaceus et T. strigilatus, Gm. T. melanopterus, Sw. Levaill. Hist. Nat. Courc. t. 3, 4. Gould, Monogr. of Trog. pl. 10, 11. 'T. leverianus, Shaw, Lev. Mus. pl. T. albiventer, Cur.

S. America (Brazil, Cayenne, Guyana), and W. Indies.

663. Trogon ramoniana.

Trogon ramoniana, Dev, et $O$. Des Murs, Rev. \& Mag. de Zool. 1849 , p. 332 .

S. America. 
664. Trogon me lanoce PHaLUS.

B. M.

Trogon melanocephalus, Gould, Monogr. of Trog. pl. 12.

Central America (Mexico, Honduras).

665. Trogon HELIOTHRIX.

Trogon heliothrix, Tschudi.

S. America (Peru).

666. Trogon assimilis.

Trogon assimilis, Gould.

S. America (Peru).

667. Trogon citreolus.

Trogon citreolus, Gould, Monogr. of Trog. pl. 13. T. chrysogaster, $S w$.

Central America (Mexicu).

668. Trogon auraytius.

B. M.

Trogon aurantius, Spix, Av. Bras. t. 36. Gould, Monogr. of Trog. pl. 14.

S. America (Brazil).

669. Trogon surucura.

B. M.

Trogon surucura, Vieill. Gould, Monogr. of Trog. pl. 15. Azarc, Hist. Nat. ᄂ. 24.

S. America (Paraguay, Brazil, Str. of Magellan (?).

670. Trogon Massena.

B. M.

Trogon Massena, Gould, Monogr. of Trog. pl. 16.

Central America (Mexico, Honduras).

671. Trogon macroura.

B. $M$.

Trogon macroura, Gould, Monogr. of Trog. pl. 17. T. macrurus, $\operatorname{Pr} . B$.

S. America (Venezuela), and Central America (Mexico). 
672. Trogon melanurus.

B. M.

Trogon curucui, L. Pl. enl. 752, 737. Levaill. Hist. Nat. Courc. t. 1, 2. T. melanurus, Sw. Gould, Monogr. of Trog. pl. 18. T. nigricauda, Gould.?

S. America (Demerara, Para).

673. Trggon roseigaster.

Trogon roseigaster, Vieill. Levaill. Courc. t. 13. Gould, Monogr. Trog. pl. 20. T. domicellus, Cuv. T. rhodogaster, Temm. Temnurus? roseigaster, Gould.

St. Domingo.

h. Priotelus, G. R. Gr. 1840.

Temuurus, $S w .1837$.

Prionotelus, Reichenb. 1850.

674. Trogon temnurus.

B. M.

Trogon temnurus, Temm. Pl. col. $326 . \quad$ Sagra, Voy. l'Isle de Cuba, t. 26. Temnurus albicollis, $S w$. Trogon (Temnurus) albicollis, Gould, Monogr. of Trog. pl. 19. Priotelus temnurus G. R. Gr.

Cuba.

c. Apaloderma, $S w .1837$.

Hapaloderma, Reichenb. 1850.

675. Trogon Narina.

B. $M$.

Trogon narina, Vieill. Levaill. Ois. d'Afr. t. 228, 229. Levaill. Hist. Nat. Courc. t. 10, 11. Apaloderma narina, $S w$. Trogon (Apaloderma) narina, Gould, Monogr. of Trog. pl. 26.

S. Africa.

d. Harpactes, Sw. 1837.

Harpalurus, Reichenb. 1850.

676. Trogon Rutilus.

B. M.

Trogon rutilus, Vieill. Levaill. Hist. Nat. Courc. t. 14. T. cinnamomeus et T. Duvaucelii, Temm. Pl. col. 291. Harpactes Duraucelii, Sw. H. rutilus, G. R. Gr. Trogon (Harpactes) Duvaucelii, Gould, Monogr. of Trog. pl, 32.

Sumatra and Borneo. 
677. Trogon Diardi.

B. M. Trogon Diardii, Temm. Pl. col. 54l. Harpactes Diardii, Sw. Trogon (Harpactes) Diardii, Gould, Monogr. of Trog. pl. 30 .

Sumatra and Borneo.

678. Trogon maLABaricus.

B. $M$ Trogon malabaricus, Gould. Harpactes malabaricus, $S w$. Trogon (Harpactes) malabaricus. Gould, Monogr. of Trog. pl. 31 .

Malabar.

679. Trogon KasUmba. B. M. Trogon fasciatus, Temm. Pl. col.321. T. kasumba, Raffl. T. flagrans, $K u h l . P$ Harpactes Temminckii, Sw. Trogon (Harpactes) Temminckii, Gould, Monogr. of Trog. pl. 29.

Sumatra, Java and Borneo?

680. Trogon ARDENs.

B. M .

Trogon ardens, Temm. Pl. col. 404. Trogon (Harpactes) ardens, Gould, Monogr. of Trog. pl. 35. Harpactes rodiosternus, Peale, U. S. Expl. Exped. viii. p. 166, pl. 41, f. 3 ?

Phillippine Islands.

681. Trogon Hodgsoni.

B. $\mathrm{M}$.

Trogon fasciatus, var. Lath. T. erythrocephalus, p. Gould. Trogon (Harpactes) Hodgsoni, Gould, Monogr. of Trog. pl. 34. Trogon (Harpactes) erythrocephalus, Sw.

India (Nepal).

682. Trogon FLAGRANS.

Trogon flagrans, Miill. Tijdsch. Nat. Gesch. 1835, t. 8, f. 2. T. erythrocephalus, Gould, Monogr. of Trog. pl. 33. Harpactes flagrans, G. R. Gr.

Sumatra and India (Birmah). 
683. Trogon oreskios.

B. M.

Trogon oreskios, Temm. Pl. col. 131. Trogon (Harpactes) oreskios, Gould, Monogr. of Trog. pl.36. Harpactes Gouldii, $S w$.

Java and Sumatra?

684. Trogon Reintwardtit.

B. M.

Trogon Reinwardtii, Temm. Pl. col. 124. T. sulphureus, Bezbie. Apaloderma Reinwardtii, $S w$. Trogon (Apaloderma) Reinwardtii, Gould, Monogr. of Trog. pl. 29. Harpactes Reinwardtii, G. R. Gr.

Java, Sumatra and Molucca.

685. Trogon Mackloti.

Trugon Mackloti, Mïl. Tijdsch. Nat. Gesch. 1835, t. 8, f. 1 Harpactes Mackloti, G. R. Gr.

Sumatra.

686 ? Trogon gigds.

Trogon gigas, Vieill. Levaill. Courc. t. 12. T. Temminckii, Cuv. Apaloderma Cuvieri, Sw. A. gigas, Gould. Harpactes grigas, $G, R . G r$.

Molucca?

687. ? Trogon INDICUS.

Trogon indicus, Lath. Harpactes? indicus, G. R. Gr. India.

e. Pharomachrus, Llave, 1801.

Calurus, Sw. 1837.

688. Trogon mocinNo.

B. M.

Pharomachrus mocinno, Llave. Trogon resplendens, Gould,

T. pavoninus, Temm. Pl. col. 372. T. paradiseus, Pr. B.

Calurus resplendens, $S w$. Gould, Monogr. of Trog. pl.

Central America (Guatimala).

689. Trogon pavoninus.

B. M.

Trogon pavoninus, Spix, Av. Bras. t. 35. Gould, Monogr. of Trog. pl.23. Calurus pavoninus, $S w$.

S. America (Brazil). 
690. Trogon auriceys. B. M. Calurus auriceps, Gould. G. R. Gr. \& Mitch. Gen. of B. pl. 25.

S. America (Sta. Fé de Bogota).

691. Trogon Fulgidus.

B. M.

Trogon (Calurus) fulgidus, Gould, Monogr. of Trog. pl. 24.

S. America (Guiana? Venezuela).

692. Trogon NEOXENUS.

Trogon (Calurus) neoxenus, Gould, Monogr. of Trog. pl. 25.

Central America (Mexico).

f. Cosmurus, Reichenb. 1850.

693. Trogon antisiands.

B. M.

Trogon antisianus, D'Orb. Mag. de Zool. 1837, Ois. t. 85. T. peruvianus, Gould, MSS. Trogon (Calurus) pulchellus, Gould, Monogr. of Trog. pl. 22. Calurus antisianus, G. R. Gr.

S. America (Peru, Sta. Fè de Bogota).

\section{ALCEDINID无.}

Alcedinidæ, $P r$. B. 1838-49; G. R. Gr. 1841-46.

Halcyonidæ, Viy. 1825; Sw. 1831-37; G. R. Gr. 1840; Bl. 1849.

Meropidæ, $\operatorname{Pr}$. B. 1831.

Cuculidæ, p. Pr. B. 1831 ; $C a b .1847$.

Capitonidæ, Selys, $1842 ;$ Pr. B. 1849-50.

Galbulidæ, Pr. B. 1831-49-50; Selys, 1842 ; Cab. 1847 ; Bl. 1849.

Jacamaciridæ, Pr. Max. 1832.

Buccouidx, Pr. Max. 1832.

Tamatiadæ, $B l .1849$.

Galbulæ, Alcyonides, Meropides et Cuculides, p. Sundev. 1835. 


\section{i. Bucconina.}

Bucconinæ, Pr. B. 1831 ; G. R. Gr. 1846 ; Cab. 1847.

Tamatianæ, G. R. Gr. $1840-41$.

Capitoninæ, Pr. B. 1849-50.

\section{Bucco.}

\section{a. Bucco, L. 1766. Tamatia, Cuv. 1817. \\ Cyphos, Spix, 1824.}

694. Bucco coldaris.

B. $\mathrm{M}$. Bucco capensis, L. Pl. enl. 395. B. collaris, Lath. Levaill. Barb. t. 42. Tamatia collaris, Cuv. Capito collaris, Temm.

S. America (Guiana, Peru).

695. Bucco macrorhynchos.

B. M.

Bucco macrorhynchos, Gm. Pl. enl. 689. Levaill. Barb. t. 39. Tamatia macrorhynchos, Cuv. Capito macrorhynchos, Temm. Cyphos macrorhynchos, Strickl.

S. America (Cayenne, Brazil, Peru), and Central America, (Honduras).

696. Bucco Swainsoni.

B. M.

Tamatia macrorhyncha, Sw. Zool. Illustr. pl. 99. Bucco Swainsoni, G. R. Gr. Capito Swainsoni, Pr. B.

S. America (Brazil).

697. Bucco pectoralis.

B. M.

Bucco pectoralis, G. R. Gr. \& Mitch. Gen. of B. pl, 26. Capito pectoralis, $\operatorname{Pr} . B$.

S. America.

698. Bucco tectus.

B. $\mathrm{M}$.

Bucco tectus, Bodd. Pl. enl. 688, f. 2. B. melanoleucos, Gm. Levaill. Barb. t. 40. Tamatia melanoleucos, Cuv. Capito melanoleucos, Wagl.

S. America (Cayenne). 
699. Bucco Bicinctus.

B. $\mathbf{M}$.

Tamatia bicincta, Gould. T. bitorquata, Sw. Bucco bicinctus, G. R. Gr. Capito bicinctus, $\operatorname{Pr}$. $B$.

S. America (Venezuela, Columbia).

700. Bucco macrodactylus.

Cyphos macrodactylus, Spix, Av. Bras. t. 39, f. 2. Capito cyphos, Wagl. C. macrodactylus, $\operatorname{Pr}$. B. Bucco macrodactylus, G. R. Gr.

S. America (Brazil, Peru).

701. Bucco gULaris.

Tamatia gularis, D'Orb. \& Lafr. Bucco gularis, G. R. Gr. Capito gularis, $\operatorname{Pr} . B$.

S. America (Venezuela).

702. Bucco Ruficolits.

Bucco ruficollis, Licht. Capito ruficollis, Wagl.

Central America (Mexico).

703. Bucco panamensis.

Malacoptila panamensis, $L a f r$. Capito panamensis, $P r . B$. Bucco panamensis, G. R. Gr.

Central America.

704. Bucco lanceolata.

Bucco lanceolata, Deville, Rev. et Mag. de Zool. 1849, p. 56.

South America.

b. Chaunomis, G. R. Gr. 1841.

Nyctactes, Strickl. 1841.

705. Becco tamatia.

B. M.

Bueco tamatia, Gm. Pl. enl. 746, f. 1. Tamatia maculata, Cuv. Chaunornis tamatia, G. R. Gr. Capilo tamatia, Steph. Nyctactes tamatia, Strickl. Sw. B. of Braz. pl. 11. Levaill. Barb. t. 41.

S. America (Brazil, Cayenne). 
706. Bucco ohacura.

B. M. Bucco chacura, Vieill. B. strigilatus, Licht. Capito chacura, Strickl. C. melanotis, Temm. Pl. col. 94. Tamatia melanotis, Cuv. T. chacura, $\mathrm{Pr}$. B.

S. America (Brazil, Peru).

707. Bucco maculatus.

B. $\mathrm{M}$.

Alcedo maculata, Gm. Levaill. Guep. Suppl. t. F. Bucco somnolentus, Licht. B. maculatus, G. R. Gr. Capito maculatus, Temm. C. somnolentus, $S w$. B. of Braz. pl.9. Tamatia tamajac, Less. T. maculatus, Pr. $\dot{B}$.

S. America (Brazil, Peru).

708. Bucco Leucotis.

Capito leucotis, Sw. B. of Braz. pl. 10. Bucco leucotis, G.R. $G r$.

S. America (Brazil).

\section{Monasa.}

a. Monasa, Vieill. 1816.

Lypornis, Wagl. 1827.

Monassa, $S w$.

Scotocharis, Glog. 1827.

Monastes, Nitzsch, 1840.

Monasta, Cab. 1848.

709. Monasa ATra. B. M.
ater, Bodd. Pl. enl. $512 . \quad$ C. tranquillus, Gm. LeLath. Corvus australis, Gm. C. affinis, Shaw. Monasa tranquilla, Vieill. M. atra, G. R. Gr. Lypornix tranquilla, Wagl. Capito calcaratus, Temm.

S. America (Guiana).

710. Monasa leucops.

B. M. Bucco leucops, Licht. B. albifrons, Spix, Av. Bras. t. 41, f. 1. Monasa leucops. Sw. B. of Braz. pl. 12. M. personata, Vieill. Gal. des Ois. t. 36. Capito leucops, Temm. Lypornix leucops, Wagl.

S. America (Brazil). 
711. Monasa nigrifrons.

B. $M$. Bucco nigrifrons, Spix, Av. Bras. t. 43, f. 2. Lypornix unicolor, Wagl. Monasa nigrifrons, G. R. Gr.

S. America (Brazil).

712. Monasa flavirostris.

Monasa flavirostris, Strickl. Jard. Contr. Ornith. 1850, pl.

S. America (Peru).

b. Malacoptila, G. R. Gr. 1841.

Lypornis, $S \boldsymbol{w}$.

713. Monasa fuscia.

B. M.

Bucco fuscus, Gm. B. torquatus, Hahn. B. striatus, Spix, Av. Bras. t. 40, f. 2. Tamatia fusca, Less. Monastes fuscus, Nitzsch. Lypornix torquata, Wagl. L. striata, Sw. B. of Braz. pl. 34. Monasa fusca, G. R. Gr. Levaill. Barb. t. 43 .

S. America (Brazil).

714. ? Monasa unitorques.

Monasa unitorques, DuBus.

S. America (Peru).

715. ? Monasa inornata.

Monasa inornata, $D u B u s$.

Central America (Guatimala).

716. Monasa Rufa.

Bucco rufus, Spix, Av. Bras, t 40, B. M.

G. R. Gr. Lypornix rufa, Wagl. 4 , f. 1. Monasa rufa, S. America (Brazil, Peru).

717. Movasa rubecuita.

B. M.

Bucco rubecula, Spix, Av. Bras. t. 39 , f. 1. Monasa rubecula, Less. M. phaioleucus, Temm. Pl.col.323, f. 2. Sw. B. of Braz. pl. 35. Lyporuix rubecula, Wagl.

S. America (Brazil). 
718. Monasa ruficapilla.

Bucco ruficapillus et Lypornix ruficapilla, Tschudi, Fauna Per. t. 24, f. 1. Monasa ruficapilla, G. R. Gr.

S. America (Peru).

719. Monasa mysticalis.

Monasa mysticalis, Lafr. Rev. \& Mag. Zool. 1850, p. 215, t. 3.

South America.

3. Chelidoptera.

Chelidoptera, Gould, 1836.

Brachypetes, Sw. 1837.

720. Chelidoptera tenebrosa.

B. 1 .

Cuculus tenebrosus, Pall. Pl. enl. 505. Pl. col. 323, f. $\mathrm{l}$.

Capito tenebrosus, Temm. Chelidoptera tenebrosa, Gould. Brachypetes tenebrosa, $S w$. B. of Braz. pl. 36. Lypornis tenebrosa, Wagl. Monasa tenebrosa, Vieill.

S. America (Brazil, Guiana).

\section{ii. Dacelonina.}

Daceloninæ, $\operatorname{Pr}$. B. 1849-50.

Alcedininæ, p. Pr. B. 1831.

Halcyuninæ, G. R. Gr. 1840-41-46; Cab. 1847.

\section{Dacelo.}

a. Dacelo, Leach, 1815 .

Paraleyon, Glog. 182\%.

Choucalcyon, Less. 1831.

721. Dacelo gigas.

B. M.

Alcedo gigas, Bodd. Pl. enl. 663. A. fusca, Gm. A. gigantea, Lath. Gal. des Ois. t. 188. A. undulata, Scop. Voy. Ind. t. 106. Dacelo gigantea, Leach, Zool. Misc. pl. 104. Gould, B. of Austr. ii. pl. 18. D. gigas, G.R. Gr. Choucalcyon australe, Less.

New South Wales and S. Australia. 
722. Dacelo Leachil.

Dacelo Leachii, Lath. Gould, B. of Austr. ii. pl. 19.

B. M.

N. Australia.

723. Dacelo Cervina.

B. NI.

Dacelo cervina, Gould, B. of Austr. ii. pl. 20. Voy. au Pole Sud.t. 23, f. 1 .

N. Australia.

724. Dacelo Gaudichaudi.

B. M.

Alcedo Gaudichaudii, Quoy et Gaim. Voy. de l'Uranie, Ois, t. 21. Dacelo Gaudichaudii, G. R. Gr. Choucalcyon Gaudichaudii, Less.

New Guinea.

725. Dacelo cyanotis.

Dacelo cyanotis, Temm. $P l$. col. 262.

B. M.

Sumatra and Celebes.

b. Melidora, Less. 1831 .

726. Dacelo MACRoRHYNCHA.

Dacelo macrorhynchus, Less. Voy. de la Coqu. t. 31, bis. f. 2.

Melidora euphrosiæ, Less.

New Guinea.

\section{HALCYON.}

a. Halcyon, Less. 1821.

Entomothera, Horsf. 1820.

Entomophila, Leach. MSS.

Paralcyon, Glog. 1827.

Cancrophaga, Pr. B. 1850.

Calialcyon, $\operatorname{Pr}$. B. 1850.

727. HaIcYon CONCRETA.

Dacelo concreta, Temm Pl. G. R. Gr. H. monacha, p. $B l$ Halcyon concreta, Sumatra. 
728. HaLcyon LindSaYi.

B. M.

Dacelo Lindsayi et D. Lessoni, Vig. Halcyon Lindsayi, G. R. Gr. \& Mitch. Gen. of B. pl, 27. Voy. de la Bonite, Ois. t. 7. H. rufula, Lafr.?

Philippine Islands.

729. HALCYON MONACHA.

Dacelo monacha, Temm. Forst. Halcyon monacha, G. R. Gr.

Celebes.

730. Halcyon PULChella.

B. $M$.

Dacelo pulchella, Horsf. Pl. col. 277. D. buccoildes, Temm. $P l$. col. 586. Halcyon pulchella, G.R. Gr.

Java and Sumatra.

731. Halcyon melanops.

Dacelo melanops, Temm. Halcyon melanops, Pr. B. Consp. p. 154 .

Borneo.

732. HALCYON MELANORHYNCHA.

Alcedo melanorhyncha, Temm. Pl. col. 391. Halcyon melanorhyncha, $G . R$. $G r$.

Celebes.

733. Halcyon pileata.

B. M.

Alcedo pileata, Bodd. Pl. enl. 673. A. albiventris, Scop. Voy.

Ind. t. 31. A. atricapilla, Gm. A. brama, Less. Cent.de Zool.

t. 8. Halcyon pileatus, G. R. Gr. H. atricapillus, Steph.

Dacelo atricapilla, Less.

Malayan Peninsula and Archipelago.

734. HaLCYON MELANOPTERA.

Alcedo melanoptera, Horsf. A. umnicolor, Temm. Pl. col. 135. A. cyanoventris, Vieill. Halcyon melanopterus, Steph. Dacelo omnicolor, Less.

Java. 
735. Halcyon leudocephala. B. M. Alcedo leucocephala, Gm. A. javanica, Shaw. Pl. enl. 757. Halcyon capensis, Jerd. H. leucocephala, Steph. H. gurial et H. amauropterus, Pears. H. brunniceps, Jerd.

India. Malayan Peninsula and Archipelago.

736. HaLCYON CANCROPHAGa.

Alcedo cancrophaga, Bodd. Pl. enl. 334. Halcyon cancrophaga, G. R. Gr.

W. Africa.

737. HaLCYON CINEREIFRONS.

B. M. Alcedo cinereifrons, Vieill. Gal. des Ois. t. 187. Halcyon torquatus, $S w . \quad H$. cinereifrons, G. R. Gr. Todiramphus cinereifrous, $B l$.

W. Africa and Fernando Po.

738. Halcyon fuscicapilla.

B. $M$.

Dacelo fuscicapilla, Lafr. Mag. de Zool. 1833, t. 18. Halcyou fuscicapilla, $G . R$. $G r$.

S. Africa.

739. Halcyon Semicerelea.

B. M. Alcedo semicrulea, Forst. $\quad P l$. enl. 356, f. 2. A. senegalensis, var. Lath. A. cancrophaga, Forst. A. erythrogaster, Temm. A. rufiventer, $S w$. B. of $W$. Afr. ii. pl. 12. Halcyon erythrogaster et H. semicærulea, G. R. Gr. H. Swainsoni, A. Smith. H. erythrorhynchus, Gould, Voy. of Beagle, pl. H. semicærulea, Kaup. H. senegaloides, A. Smith, Ill. $\widehat{\text { S. }}$ Afr. Zool. pl. 63. Dacelo acteon, Less.

Africa.

740. Halcyon SENEgalensis.

B. M. Alcedo senegalensis, $L$. Pl. enl. 594. Halcyon senegalensis, Sw. Zool. Illustr.pl. 27. Dacelo senegalensis, Less.

W. Africa.

741. Halcyon cranoledca.

Alcedo cyanoleuca, Vieill. Halcyon cyanoleuca, G. R. Gr. Hartl.

W. Africa (Angola). 
742. HALCYON CHELICUTI.

B. M.

Alcedo chelicuti, Stanl. A. variegata, Vieill. Halcyon chelicuti et H. variegata, G. R. Gr. Dacelo pygmæa, Cretschm.? Rüpp. Atl. Zool. t. 28, b.

N.-E. and W. Africa.

743. Halcyon striolata. Alcedo striolata, Licht.

B. M.

W. Africa.

744. Halcyon SMYRNENSIS. Alcerlo smyrnensis, $L$. Halcyon smyrnensis, Sleph. Dacelo smyrnensis, Less.

Asia Minor.

745. Halcyon FusCa.

B. M.

Alcedo fusca, Bodd. $\quad$ Pl. enl. 894. A. smyrnensis, var. Lath. A. smyrnensis, var. albogularis, Bl.? A. rufirostris, Licht. Kittl. Kupf. Vog. t. 14, f. 2 ? A. macroura, Merr. Halcyon fusca, G. R. Gr.

India and Malayan Peninsula.

746. HALCYON GULARIS.

Alcedo gularis, $K u h l$. $\quad P l$. enl. 232. A. melanoptera, Temm. Halcyon ruficollis, $S w$. H. gularis, G. $R$. $G r$.

India and Malayan Peninsula.

747. HaLCYON COROMANDELIANa. B. M. Alcedo coromandeliana, Scop. Sonn. Voy. t. 118. A. coromauda, Lath. Halcyon coromandeliana, G. R. Gr. H. coromandus, Steph. H. coromandelicus, Vig. H. corumanda minor, Temm. \& Schl. H. coromanda major, Temm. \& Schl. Fauna, Jap. t. 39. Halcyon (Calialcyon) Jilacina et H. Schlegeli, $\operatorname{Pr} . \mathbf{B}$. Dacelo coromanda, Less.

India and Malayan Peninsula.

748. HaLCYON CAPENSIS.

Alcedo capensis, L. Pl. enl. 590. Halcyon capensis, $G$. R. Gr.

S. Africa. 
b. Todiramphus, Less. 1828.

749. HaLcyon tUTA. Alcedo tuta et A. sacra? Gm. A. collaris, Forst. Todiramphus sacer et T. divinus, Less. Mem. Soc. d'Hist. Nat. Paris, iii. t. $11,12$.

Society Islands.

750. ? HALCYON NULLITORQUIS.

Dacelo nullitorquis, Peale, U.S. Expl. Exped. viii. p. 150, pl. 42, f. 1.

Society Islands (Otaheite).

751. Halcyon venerata.

Alcedo venerata, $G m$. Halcyon venerata, G. R. Gr. Todiramphus veneratus, $\operatorname{Pr} . B$.

Friendly Islands.

752. HaLCXON SUPERCILIOSA.

B. $M$.

Dacelo albicilla, var. Less. Halcyon superciliosa, G. R. Gr. \$. Mitch. Gen. of B. pl. 27, f. 4.

Friendly Islands (Tongataboo).

753. HaLcyon vitiensis.

Dacelo vitiensis, Peale, U. S. Expl. Exped. viii. p. 156, pl. 44.

Feejee Islands (Orolau, Venua-levu).

754. Hatcyon Platyrostris.

B. $M$. Halcyon platyrostris, Gould. Todiramphus recurvirostris, Lafr. T. platyrostris, $\operatorname{Pr}, B$. Dacelo minima, Peale, U. S. Expl. Exped. viii. p. 159, pl. 45.

Navigators' Islands (Upolu).

755. Halcyon Pealei.

Dacelo coronata, Peale, U. S. Expl. Exped. viii. p. 160, pl. 46.

Navigators' Islands (Tutuila). 
756. HaLCYON SANCTA.

B. M. Halcyon sanctus, Vig. \& Horsf. Gould, B. of Austr. ii. pl. 21. H. sacra, Steph. Dacelo chlorocephala, var. Less. Alcedo australasiæ, Vieill. Todiramphus sanctus, $\operatorname{Pr} . B$. T. sacer, Bl. Phillips'Bot. Bay, pl. in p. 156 . Jard. \& Selby's Ill. Orn. pl. 96.

Australia (except Van Diemen's Land).

757. HaLCYON COLLARIS.

Halcyon collaris, Sw. Zool. Illustr. pl. 27. Todiramphus collaris, $B$.

Philippine Islands? Malayan Arehipelago.

758. HALCYON CHLORXS.

B. M. Alcedo chlcris, Bodd. Pl. enl. 783, f. 2. A. chlorocephala, Gm. A. collaris, Scop. Sonn. Voy. t. 33. Halcyon chloris, G. R. Gr. H. occipitalis, Bl.? 'Todiramphus collaris et T. occipitalis, $B l$. Todiramphus chlorocephalus, $\operatorname{Pr}, B$. Kittl. Kupf.Vog. t. 14, f. 1.

India; Malayan Peninsula and Archipelago.

759. HaLcYoN TARIA.

Halcyon varia, Eyton. Todiramphus varius, $B l$.

Malayan Peninsula and Archipelago.

760. HALCYON SORDIDA.

B. M. Halcyon sordidus, Gould, B. of Austr. ii. pl. 23. Todiramphus sordidus, $\operatorname{Pr} . B$.

N. Australia.

761. Halcyon vagans.

B. $\mathrm{M}^{*}$

Alcedo sacra, var. Lath. A. cyanea, Forst. A. ragans, Less. Dacelo chlorocephala, var. $\gamma$. Less. Halcyon vagans, G. $R$. Gr. Voy. Ereb. \& Terr. pl. 1. H. cinnamominus, Su. Zool. Illustr. pl.67. Todiramphus vagans et T. cinnamominus, $B l$.

New Zealand.

762. HaLCYON CORONATA.

Halcyon coronata, Müll. Verh. Nat. Gesch. Ned. p. 175. Todiramphus coronatus, $\operatorname{Pr} . B$.

Timor. 
763. HALCYON PYRRHOPYGIA, B. M. Halcyon pyrrhopygia, Gould, B. of Austr, ii. pl.22. Todiramphus pyrrhopygius, $B l$.

New South Wales; W. and S. Australia.

764. Halcyon MacLeayit.

B. $M$.

Halcyon MacLeayii, Jard. \& Selby, Illustr. of Orn. pl. 101. H. incinctus, Gould, B. of Austr. ii. pl. 24. Todiramphus MacLeayii et T. incinctus, $B l$.

New South Wales and N. Australia.

765. HALCYON DIOPS.

Alcedo diops, Temm. Pl. col. 272. Halcyon diops, G. R. Gr. Todiramphus diops, $\mathrm{Pr}$. B.

Amboina, Timor and Celebes.

766, Halcyon lazuli.

Alcedo lazuli, Temm. Pl. col.500. Halcyon lazuli, G. R. Gr. Todiramphus lazuli, $\operatorname{Pr} . B$.

Sumatra.

767. HaLCYON fUNEBRIS.

Halcyon funebris, Forst. Todiramphus funebris, $\operatorname{Pr}$. B. Consp. p. 157.

Celebes.

768. Halcyon Forsteni.

Halcyon Forsteni, Temm. Todiramphus Forsteni, Pr. B. Consp. p. 157.

Celebes.

769. HaLCYoN ALBICILLA.

Alcedo albicilla, Cuv. A. cyanoleuca, Temm. Dacelo albicilla, Less. Halcyon albicilla, G. R. Gr. H. saurophaga, Gould, Voy. of Sulphur, Birds, pl. 19. Todiramphus saurophagus, $\boldsymbol{P r}$. $B$.

New Guinea. 
c. Actenoïdes, Homb. \&. Jacq. 184 ?.

770. Halcyon actenoides.

Halcyon actenoïdes, Pr. B. H. (sp. 47). G. R. Gr. Actenoïdes Hombroni, Pr. B. Voy. au Pole Sud, Ois. t. 23, f. 2. ?

\section{d. Syma, Less. 1828.}

771. HaLCYON TOROTORO.

Halcyon torotoro, Less. Voy. de la Coqu. t. 31, bis. f. 1. Syma torotero, Less. S. Lessonia, Sw. Dacelo syma, Miill.

New Guinea.

772. HaLCYON FLAVIrostris.

B. M.

Halcyon (Syma?) flavirostris, Gould.

N. Australia (Cape York).

e. Tanysiptera, Vig. 1823 .

773. HALCYON DEA.

Alcedo dea, $L$. Pl. enl. 116. Tanysiptera dea, Vig.

B. M. Moluccas and New Guinea.

774. Halcyon SYluia.

B. M.

Tanysiptera sylvia, Gould.

N. Australia (Cape York).

775. Halcyon Nympha.

Tanysiptera Nympha, G. R. Gr.

B. M. - ?

\section{Ceyx.}

Ceyx, Lacep. 1800-1801.

776. Ceyx tridactyla.

B. M. Alcedo tridactyla, $L$. Sonn. Voy. t. 32. Ceyx tridactyla, Cuv. C. luzoniensis, Steph.

India and Malayan Archipelago. 
777. Ceyx rubra.

B. M.

Alcedo rubra, Bodd. Pl. enl. 778, f. $2 . \quad$ A. purpurea. Gm. Jerd. Ill. Ind. Orn. pl. 25. Ceyx rubra, G. R. Gr. C. purpurea, Less. C. microsoma, Burt. C. erythaca, Bl.

Philippine Islands.

778. Ceyx Rufidorsa.

Ceyx tridactyla, Jard. \& Selby, Ill. Orn. pl. 55, f. 2. C. rufidorsa, Strickl. Alcedo madagascariensis, Lath.?

Malayan Peninsula and Archipelago.

779. Ceyx melanura.

Cevx malanura, Kaup.

B. M.

Malayan Archipelago.

780. Ceyx lepida.

Alcedo lepida, Temm. Alcyone lepida, G. R. Gr. Ceyx lepida, Temm. Pl.col.595, f. 1 .

Amboina.

iii. Alcedininæe.

Alcedininæ, G. R. Gr. 1840-41-47; Pr. B. 1849-50.

Halcroninæ, Sw. 1831.

Alcedininæ, p. $\operatorname{Pr}$. B. 1831.

\section{Alcedo.}

a. Alcedo, L. 1756.

Ispida, $L .1735$.

781. Alceno ispida.

B. M.

Alcedo ispida, L. Pl. enl. 77. Yarr. Brit. B.2 edit. ii. fig. p. 223. A. subispida et A. advena, Brehm. Gracula Atthis, Gm. Turdus Atthis, Steph. Kittl. Kupf. Vög. t. 29, f. 1. Gould, B. of Eur. pl. 61.

England ; Europe, India (Afghanistan?). W. and N. Africa. 
782. Alcedo bengalensis.

B. M.

Alcedo bengalensis, Gm. Edw. B. pl. 11. A. hispidioïdes

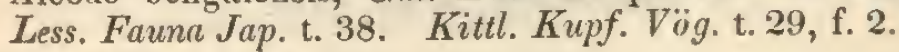

India, Malacca and China.

783. Alcedo Moludcensis.

Alcedo moluccensis, $B l$.

Moluccas and Celebes.

784. Alcedo euryzona.

B. M.

Alcedo euryzona, Temm. A. nigricans, $B l$.?

Malayan Archipelago.

785. Alcedo meninting.

B. M.

Alcedo meninting, Horsf. $\quad P l$. col. 239, f. 2. A. asiatica, $S w$. Zool. Illustr. pl.50. Ceyx meninting, Cuv.

Malayan Peninsula and Archipelago.

786. AlCEDo BIRU.

B. M.

Alcedo biru, Horsf. $P l . c o l .239$, f. 1.

Java.

787. Alcedo SEMitorquata.

B. M.

Alcedo semitorquata, Sw. Illustr. Zool. pl. 151. Rüpp. Syst. Uebers. Vög. t. 7.

W. and N.-E. Africa.

788. Alcedo leucogaster.

B. $\mathrm{Mr}$.

Halcyon leucogaster, Fras. Zool. Typica, pl. Alcedo leucogaster, Kaup.

Fernando Po.

789. Alcedo quadribrachys.

Alcedo quadribrachys, Temm. Pr. B. Consp. p. 158.

W. Africa. 
b. Corythornis, Kaup, 1848.

790. Alcedo c.eruleocephala.

B. M.

Alcedo cæruleocephala, $\mathrm{Gm} . \quad \mathrm{Pl}$. enl. $356, \mathrm{f} .1 . \quad$ A. cyanocephala, Shaw. Alcedo (Corythornis) cæruleocephala, Kaup.

S. and W. Africa and Madagascar.

791. Alcedo cyanostigma.

B. M.

Alcedo cyanostigma, Rüpp. Fauna Abyss. t. 24, f. 2.

(Corythornis) cyanostigma, Kaup.

N.-E. Africa.

792. A lCE do NaIS.

Alcedo nais et A. (Corythornis) nais, Kaup.

Alcedo

Africa.

793. Alcedo cristata.

B. M.

Alcedo cristata, $L$. Pl. enl. 756, f. 1. Alcedo (Corythornis) cristata, Kaup. Kittl. Kupf. Vög. t. 29, f. 3.

W. Africa.

794. A lCEDO vintsioides.

B. M.

Alcedo vintsioïdes, Eyd. \& Gerv. Voy. de la Favor. Mag. de Zool. 1836, Ois. t. 74. Edw. B. pl. 336? Alcedo (Corythornis) vintsioïdes, Kaup.

Madagascar.

c. Ispidina, Kaup, 1848.

795. Alcedo picta.

B. $M$.

Todus pictus, Bodd. Pl. enl. 783, f. 1. Alcedo cærulea, Gm. A. capistrata, Shaw. A. ultramarina, Daud. Shaw, Nat. Misc. pl. 901. A. pusilla, Shaw, Nat. Misc. pl.159. Halcyon cyanotis, Sw. Alcyone coronata, A. Smith. Alcedo (Ispidina) picta, Kaup. A. (Ispidina) cyanotis, $P r, B$.

W. Africa. 
796. Alcedo madagascariensis.

Alcedo madagascariensis, $L$. Pl. enl. 778, f. 1. Alcedo (Ispidina) madagascariensis, Kaup.

Malayan Peninsula and Archipelago.

797. Alcedo cerrulea.

Alcedo cærulea, Gm. Briss. Orn. iv. t. 37, f. 2, A. A. grandis, Bl.P Alcedo (Ispidina) cærulea, $P r . B$.

India.

798. Alcedo nitida.

B. M

Alcedo nitida, Kaup. Alcedo (Ispidina) nitida, Kaup.

W. Africa.

\section{AlcYone.}

Alcyone, $S w .1837$.

Halcyone, Agass.

Therosa, Miill.

799. Alcyone azurea.

B. M.

Alcedo azurea, Lath. Jard. \& Selby, Ill. Orn. pl. 55, f. 1. Gould, B. of Austr. ii. pl. 25. Ceyx azurea, Steph. C. tribrachys, Cuv. C. cyanea, Less. Alcyone azurea, G. R. Gr. A. australis, Sw. Zool. Illustr. pl. 26. A. tribrachys, Shaw, Nat. Misc. pl.681. A. pulchra et A. diemensis, Gould.

Australia (except the West).

800. Alcyone solitaria.

Ceyx solitaria, Temm. Pl. col.595, f. 2. Therosa solitaria, Müll. Alcyone solitaria, G. R. Gr.

New Guinea.

801. Alcyone pusilla.

Ceyx pusilla, Temm. Pl. col. 595, f. 3. Gould, B. of Austr. ii. pl. 26. Alcyone pusilla, Gould.

New Guinea and N. Australia.

802. Alcyone cyanipectus.

Ceyx cyanipectus, Lafr. Alcyone cyanipectus, $\operatorname{Pr}$. B. A. cincta, Jard. Contr. Ornith. $1850, \mathrm{pl}$.

Philippine Islands. 


\section{Ceryle.}

Ceryle, Boie, 1828.

Ispida, $S w .1837$.

803. Certhe rudis.

B. M.

Alcedo rudis, $L . \quad P l$. enl. 716, 62. Edw. B. pl.9. Ceryle rudis, Boie. C. varia, Strickl. Ispida bicincta et I. bitorquata, $S w$.

S.-E. Europe, Asia and Africa.

804. Céryle guttata.

B. M. Alcedo guttata, Vig. Gould, Cent. of B. pl. A. lugubris, Temm. Pl. col.548. Fauna Jap. t. 38, B. Ceryle guttata, G. R. Gr.\& Pr. B. C. lugubris, $\operatorname{Pr} . B$.

India (Himalayah Mountains) and Japan.

805. Ceryle maxima.

B. M.

Alcedo maxima, Pall. Pl. enl. 679. A. guttata, Bodd. A. afra, Shaw. Ispida gigantea, $S w$. Ceryle maxima, $G$. R. Gr.

W. Africa.

806. Ceryle superciliosa.

B. $\mathrm{M}$. Alcedo superciliosa, $L . \quad E d w . B$. pl. 245. Pl. enl. 756, f. 3 . Ceryle superciliosa, G. R. Gr.

S. America (Guiana).

807. Cerrye alcyon.

Alcedo alcyon, L. Pl. enl. 715. A. guacu, Vieill. Ceryle alcyon, Boie. Ispida alcyon, Sw. Wils. Amer. Orn. pl. 23, f. 1. Audub. B. of Amer. pl. 77.

Ireland! N. America.

808. Cervle torquata.

B. M. Alcedo torquata, L. Pl. enl. 284. A. cinerea, Bonn. A. cyanea, Vieill. A. stellata, Meyen, Nova Acta, Bonn, ii. t. 14, Suppl. Ceryle torquata, G. R. Gr.

Central America (Mexico) and S. America (Guiana, Peru). 
809. Cermle amazona.

B. M.

Alcedo amazona, Lath. A. rubescens, Vieill. Ceryle amazona, Boie.

S. America (Guiana, Peru).

810. Ceryle viridirufa.

B. M.

Alcedo viridirufa, Bodd. Pl. enl.592, f. 1,2. A. bicolor, Gm. Ceryle viridirufa, G. R. Gr. C. bicolor, Boie.

S. America (Guiana).

811. Certele americana. B. M. Alcedo americana, Gm. Pl.enl.591. A. viridis, Vieill. Ceryle americana, Boie.

S. America (Guiana).

812. Ceryle Cabanisi.

Alcedo americana et A. Cabanisi, Tschudi.

S. America (Peru).

813. ? Cermle inda.

Alcedo inda, L. $\quad$ Edw. B. pl. 335. Shaw, Nat. Misc. pl. 889. Ceryle? inda, G.R. Grr.

S. America (Guiana).

814. ? CERYLE LEUCORHYNCHA.

Alcedo leucorhyncha, Gm. Seba, Thes. i. t.58, f. 3. Ceryle?? America.

leucorhyncha, G.R. Gr.

815. ? Ceryle cayanensis.

Alcedo cayanensis, Gm. A. taparara, Bonn. Ceryle ?? cayanensis, $G . R . G r$.

S. America (Guiana).

816. ? Ceryle brasiliensis.

Alcedo brasiliensis, Gm. Ceryle?? brasiliensis, G. R. Gr .

S. America (Brazil). 
817. Ceryle surinamensis.

Alcedo surinamensis, $G m$. Ceryle?? surinamensis, G. R. Gr.

S. America (Guiana).

818. ? Ceryle tricolor.|

Alcedo tricolor, Vieill. Ceryle (?) tricolor, Hartl.

W. Africa (Senegal).

iv. Galbuline.

Galbulinæ, G. R. Gr. 1840-41-47; Cab. 1847; Pr. B. 1849 -50 .

\section{Galeula.}

a. Galbula, Mrchr, 1752 .

819. Galbula viridis.

B. M.

Alcedo galbula, $L, \quad P l$. enl.238. Edw. B. pl. 334. Galbula viridis, Lath. G. viridicauda, Sw. G. rubricollis, Shau. Levaill. Jacam. t. 47, 48, 49 . Vieill. Ois. dor. t. 1, 2.

S. America (Guiana, Brazil).

820. Galbela ruficauda.

Galbula ruficauda, Cuv. Levaill. Jacam. t. 50.

B. M.

S. America (Brazil, Columbia).

821. Galbula macroura.

B. M.

Galbula macroura, Vieill. Gal. des Ois. t. 29.

S. America.

822. Galbula leptura.

B. M.

Galbula leptura, Sw. Lath. Gen. Syn. ii. pl. 26.

S. America (Brazil). 
823. Galibula levcogastra.

B. M.

Galbula leucogastra, Vieill. Levaill. Jacam. Suppl. t. H. G. albiventer, Cuv. G. albiventris, Less.

S. America.

824. Galbula albirostris.

Galbula albirostris, Lath. Levaill. Jacam. t. 51. G. flavirostris, Vieill. G. flavirostra, Sw. Ois. dor. t. 4, 5 .

S. America (Guiana).

825. Galbula lugubris.

B. $M$.

Galbula lugubris, $S w . \quad$ G. albigularis, Spix, Av. Bras. t. 57,

f. 1. Jacamaralcyon lugubris, $G . R$. Gr.

S. America (Guiana, Brazil).

826. Galbula paradisea.

B. M.

Galbula paradisea, Lath. Alcedo paradisea, L. Pl. enl. 271. Ois. dor. t. 3. Levaill.Jamar. t. 52.

S. America (Cayenne).

827. Galbula tombacea.

Galbula tombacea, Spix, Av. Bras. t. 58.

S. America (Brazil, Peru).

828. Galbula chalcocephala.

Galbula chalcocephala, Deville, Rev. \& Mag. de Zool. 1849, p. 55.

S. America.

829. Galbula cyanescens.

Galbula cyanescens, Deville, Rev. \& Mag. de Zool. 1849, p. 56.

S. America.

b. Jacamaraleyon, Cuv. 1829.

Cauax, $C a b .1847$.

830. Galbula tridactyla.

B. $M$.

Galbula tridactyla, Vieill. Levaill. Guêp. Suppl. t. L. G. ceycoïdes, Such. G. armata, $S w$. Jacamaralcyon brasiliensis, Less. J. tridactyla, G. R. Gr. Cauax tridactyla, Cab. Jard. \& Selby, Illustr. Orn. pl. 22. Spix, Av. Bras. t. 52, f. 2 .

S. America (Brazil). 


\section{Galbulcyrhynchus.}

Galbulcyrhynchus, O. Des Murs, 1845.

Jacamaralcyonides, O. Des Murs, 1849.

831. Galbulcyrhynchus leucotis.

Galbulcyrhynchus et Jacamaralcyonides leucotis, O. Des Murs, Icongr. Ornith. pl. 17.

S. Anerica (Sta. Fé de Bogota).

\section{JACAMEROPS.}

Jacamerops, Cuv. 1817.

Lamprotila, $S w .1837$.

832. JACAME ROPS GRAN DIS.

B. $M$.

Alcedo grandis, Gm. Levaill. Jacam. t. 54. Galbula jacamarici et G. grandis, Shaw, Nat. Mise. pl. 833. Jacamerops grandis, Cuv. Lamprotila platyrhyncha, $S w$.

S. America (Surinam, Brazil).

\section{JACAMEROPS ISIDORI.}

Jacamerups Isidori, Deville, Rev. \& Mag. de Zool. 1849, p. 53.

S. America.

834. ? JaCamerops Boersil.

Galbula Boersii, Ranz. Levaill. Jacam. t. 53. Jacamerops Boërsii, G. R. Gr.

S. America.

\section{MEROPID压.}

Meropidæ, Leach, ; Vig. 1825; Sw. 1831-37; Pr. B. 1831 -38-49-50; G.R. Gr. 1840-41; Cab. 1847; Bl. 1849.

i. Meropina.

Meropinæ, Pr. B. 1831-49-50; G. R. Gr. 1846 ; Cab. 1847. 


\section{Merops}

a. Merops, L. 1756. A piastur, Br. 1760

Ispida, p. L. 1735 .

835. Merops apiaster.

B. M.

Merops apiaster, $L . \quad P l . e n l .938 . \quad$ M. chrysocephalus, $G m$. M. schœghagha, Forsk. M. hungariæ, Brehm. Gould, B. of Eur. pl. 59. Yarr. Brit. B. 2 edit. ii. fig. p. 217.

England; Europe, India (Afghanistan) and N. Africa.

836. Merops agrptius.

B. M.

Merops ægyptius, Forsk. M. persicus, Pall. M. Savignyi, Cuv. M. ruficollis, Vieill. Levaill. Guêp. t. 6, 6*, 16. M. superciliosus, Licht. Kittl. Kupf. V̈̈g. t. 7, f. 1. Pr, B. Fauna Ital. t. 25, f. 1. B. of W. Afr. pl. 7. M. Vaillantii, Pr. B. Europe, Africa and Madagascar.

837. Merops superciliosus.

Merops superciliosus, L. Pl. enl. 259. Levaill. Guêp. t. 19. M. Bonelli, Lev. M. patirich et M. ruficapillus, Vieill.

Africa and Madagascar.

838. Merops albicollis.

B. M. Merops albicollis, Vieill. Levaill. Guêp. t. o. M. Cuvieri, Licht. M. Savignyi, Sw. Zool. Illustr. pl. 7.5.

W. Africa.

839. Merops nubicus.

B. M.

Merops nubicus, $\mathrm{Gm} . \quad \mathrm{Pl}$. enl. 649. M. cæruleocephalus, Lath. M. superbus, Penn. Shaw, Nat. Misc. pl. 78, 613. Levaill. Guêp. t. 3. Sw. B. of W. Afr. pl. 9.

W. Africa.

840. Merops nubicoides.

Merops nubicoïdes, O. Des Murs, Icongr. Omith, pl. 35. Africa. 
841. Merops malimbicus.

Merops bicolor, Daud. Ann. du Mus. ii. t. 62, f. 1. M. malimbicus, Shaw, Nat. Misc. pl. 71. Vieill. Gal. des Ois. t. 186.

E. Africa.

\section{Merops Lamarki..}

Merops viridis, p. L. Pl. enl. 740. Briss. Orn. t. 42, f. 2. M. Lamarkii, Cuv. Levaill. Guêp. t. 10. M. viridissimus, Sw.

Africa and Madagascar.

843. Merops viridis.

B. M.

Merops riridis, p. L. $E d w . B$. pl. 183 . M. orientalis, M. torquatus et M. coromandus, Lath. M. torquatus et M. ferrugiceps, Hodgs. M. indicus, Jerd. M. luteus, Scop.? M. citrinella, Vieill.P M. flavicans, Gm. Sonn. Voy. t. 119. Levaill. Guêp. t. 11.?

India, Burmah and Ceylon.

844. Merops ornatus.

B. M.

Merops ornatus, Lath. Levaill. Guêp. t. 4. M. melanurus, Lewin, B. of N. Holl.pl.2. M. Thouini, Kuhl. Philemon ornatus, Vieill. Levaill. Guêp. t. 4. Gould. B. of Austr. ii. pl, 16 .

Australia (except Van Diemen's Land).

845. Merops bicolor.

B. M.

Merops bicolor, Bodd. M. badius, Gm. Pl, enl. 252. M. castaneus, Lath. M. sumatranus. Rafh, M. Latreillii, Vieill. M. cyanopygius, Less. Levaill. Guêp.t. 12.

Malayan Archipelago.

846. M MEROPS SENEGALENSIS.

Merops senegalensis, Shaw. Pl. enl. 314. M. longicauda,

Vieill. M. Adansoni, Levaill. Guêp. t. 13.

W. Africa (Senegal). 
847. ? MEROPS ERYTHROCEPHALUS.

Merops erythrocephalus, $\mathbf{G m}$. Briss. Orn. iv. t. 44, f. 3. Shaw, Nat. Misc. pl. 357.

India.

848. Merops Javanicus.

Merops javanicus, Horsf. M. cyanorrhous et M. Savignyi, Temm.

Java.

849. Merops philippinus.

B. M.

Merops phiippinus, $L$. Pl. enl. 57. M. Daudini, Cuv. M. typicus, Hodgs. Levaill. Guêp. t. 14.

Philippine Islands; India (Nepal).

850. Merops quinticolor.

B. M.

Merops quinticolor, Vieill. Levaill. Guêp. t. 15, 18. M. urica, Horsf. M. erythrocephalus, Bl. Sw. Zool. Illustr. pl. 8. M. Leschenaultii, Vieill.

Java, India, Malayan Peninsula and Ceylon.

851.? Merops angolensis.

Merops angolensis, Lath.

W. Africa.

b. Melittophagus, Boie, 1828.

Nyctiornis, p. Pr. B. 1850 .

852. Merops ertthropterus.

B. M.

Merops erythropterus, Gm. Pl. enl. 318. M. minutus, Vieill. M. minulus, Cuv. M. pusillus, Sw. Melittophagus erythropterus, Boie. Levaill. Guêp. t. 17. Lath. Gen. Syn. pl.31.

W. Africa.

853. Merops tariegatus.

B. M.

Merops Sonnini, M. variegatus, et M. collaris, Vieill. Levaill.

Guêp.t. 7. Melittophagus variegatus, G.R.Gr. Briss. Orn.

t. 44, f. 1. Kittl. Kupf. Vög. t. 7, f. 3.

N.-E. Africa. 
854. ? Merops Lafresnayi.

Merops Lafresnayi, Guer. M. Lefebvrei, O. Des Murs, Icongr. Ornith. t. 34.? Melittophagus Lafresnayi, $\boldsymbol{P r}, \boldsymbol{B}$.

N.-E. Africa.

855. Merops hirundinaceus.

B. M.

Merops hirundinaceus, Vieill. M. taiva, Cuv. M. chrysolaimus, Jard. \& Selby, Ill. Orn. pl. 99. M. furcatus, Stanl. M. azuror, Less. Sw. B. of W. Afr. ii. pl. 10. Levaill. Guêp. t. 8. Melittophagus taiva, Boie. M. hirundinaceus, G.R. Gr.

S. Africa.

856. Merops Bullockit.

B. M.

Merops Bullockii, Vieill. Donov. Nat. Repos. pl. 45. M. cyanogaster, Sw. B. of W. Afr. pl. 8. Levaill. Guêp. t. 20. Melittophagus Bullockii, G. R. Gr.

S. Africa.

857. Merops Bullockoides.

B. MI.

Merops Bullockoïdes, A. Smith, Ill. S. Afr. Zool. pl. 9. Melittophagus Bullockoïdes, G. R. $G r$.

S. Africa.

858. Merops gularis.

B. M.

Merops gularis, Shaw, Nat. Misc. pl. 337. Melittophagus gularis, G. R. Gr. Nyctionis gularis, $\operatorname{Pr} . \boldsymbol{B}$.

W. Africa.

$$
\text { c. Meropogon, Pr. B. } 1850 .
$$

S59. Merops Forsteni.

Merops Forsteni, Temm. Meropogon Forsteni, Pr. B. Consp. p. 164 .

Celebes.

2. Nyctionnis.

Nrctiornis, Sw. 1831.

Aľcemerops, J. Geoffr. 1832.

Bucia (1836) et Napophila (1841), Hodgs. 
860. Nyctionnis Athertoni.

B. M.

Merops Athertoni, Jard. \& Selby, Ill. Orn. pl. 53. M.? cranogularis, Jerd. Nyctiornis Athertoni, Hodgs. N. cærulea, Sw. N. Amherstianus, Royle. Bucia nipalensis, Hodgs. B. Athertonii, Bl. Napophila meropina, Hodgs. N. Athertonii, $B l$.

India (Nepal, Assam) and Malayan? Peninsula.

861. NyCTIORNIS AMICTA.

B. M. Merops amictus, Temm. Pl. col. 310. Nyctiornis amicta, Sw. Zool. Illustr. n. s. pl. 56.

Malayan Peninsula. 


\section{Tribe 2. TENUIROSTRES.}

Picæ, p.Linn. 1735 ; Mœhr, 1752 ; Lath. 1790.

Plumipedes, p. Schaff. 1774.

Fissipedes anisodactyles, cuneirostres-F. a. filirostres-F. a. falcirostres, Schaff. 1774.

Scutipedes, p.-Negliges, p. Scop. 1777.

Tenuirostres, p.-Dentirostres, p.-Plenirostres, p. Cuv. $1799-$ 1800.

Tenuirostres-Gregarii, p.-Pygarrhichi-Suspensi-Gallinacei, p. Illiger, 1811.

Anthomysi-Epopsides-Anerpontes-Lyriferi, Vieill. 1816.

Scansores, p. Temm. 1815.

Tenuirostres-Conirostres, p.-Dentirustres, p. Cuv. 1817.

Anisodactyli-Insectivores, p. Temm. 1820.

Anerponti-Antomisi-Epopsidi-Rafioramfi-Metrioramfi, Ranz. 1823.

Tenuirostres-Scansores, p.-Rasores, p. Vigors, 1826.

Fissirostres, conirostres anisodactyles, p. Less. 1831.

Tenuirostres-Anthomyzi-Canori, p. Pr. Bonap. 1831.

Volucres, p. $-V$. oscines, p.-V. coraces, p.-Gessores, p. Sundev. 1835.

Scansores, p.-Oscines, p. Keys. \& Bl. 1840.

Raptatrices, Macgill. 1840.

Clamatores, p.-Oscines, p. Cabanis, 1847.

\section{UPUPIDÆ.}

Upupidæ, Pr. B. 1838-49-50; G. R. Gr. 1840-41-47;Cab. $1847 ; B l .1849$.

Promeropidæ, Steph. 1826; Sw. 1831-37; Pr. B. 1849-50.

Trochilidæ, p. Pr. B. 1831 .

Paradiseidæ, p. Sw. $1837 ;$ Cab. 1847.

Bucerotidæ. p. Bl. 1849.

Epimachidæ, Selys, 1842.

Meliphagidæ, p. Vigors, 1825.

Epimachides, Sundev. 1835.

Irrisoridæ, Pr. B. 1850. 
i. UPUPINA.

Upupinæ, Pr. B. 1831-49-50; G.R. Gr. 1840-41-47; Cab. 1847.

Meropinæ, p. Pr. B. 1831.

Promeropinæ, G. R. Gr. 1840 ; Pr. B. 1850.

Irrisorinæ, $B l$. $1849-50$.

\section{UPUPA.}

a. Upupa, L. 1735.

862. Upupa Epops.

Upupa Epops, $L . \quad P l . e n l .52 . \quad E d w . B . p l .345 . \quad$ U. vulgaris, Pall. U. bifasciata, Brehm. U. macrorhyncha, Landb. Gould, B. of Eur. pl. 238. Naum. Vög. Deuts. v. t. 142. Yarr. Brit. B. 2 edit. fig. p. 175. Levaill. Prom. t. 22.

Great Britain ; Europe, N.-W. Asia, and N. Africa.

863. UPUPA MINOR.

B. M.

Upupa minor, Shaw. U. africana, Bechst. U. capensis, $S w$. U. cristatellus, Vieill. Gal. des Ois. t. 184. Ois. dor. t. 2. Jard. Ill. of Orm. pl. 142.

S. and W. Africa.

864. UPUPA MOXOLOPHOS.

Upupa monolophos, Wagl. Levaill. Prom. t. 9 vel 23. U. senegalensis, $S w . ?$

W. Africa (Congo).

\section{b. Fregilupus, Less. 1831.}

865. UPUPA VARIA.

Upupa varia, Bodd. $P l$. enl. 697. U. capensis, $G m$. U. madagascariensis, Shaw. Levaill. Prom. t. 18. Vieill. Ois. dor. Prom. t. 3. Pastor? capensis, Temm. P. upupa, Wagl. Coracia cristata, Vieill.

S. and E. Africa ; Madagascar. 


\section{IRRISOR.}

a. Irrisor, Less. 1831.

866. IRRisor ERYTHRORHYNCHUS.

Upupa erythrorhynchos, Lath. U. viridis, Licht. Levaill. Prom. t. 1, 2, 3. Vieill. Ois. dor. t. 6. Promerops erythrorhynchos, Shaw, Nat. Misc. pl. 533. Falcinellus erythrorhynchos, Vieill. Irrisor capensis, Less. I. erythrorhynchos, G. R. Gr. Epimachus erythrorhynchus, Temm. Nectarinia ery throrhynchus, Licht.

S. Africa.

867. IRRISOR MELANORHYNCHUS.

B. M. Epimachus melanorhynchus, Wagl. Levaill. Prom. t. 4. Falcinellus senegalensis, Vieill. Nectarinia melanorhynchos, Licht. Irrisor melanorhynchus, G. R. Gr. \&.Mitch. Gen. of $B$. pl. 31. Promerops senegalensis, $S w$.

W. Africa.

868. IRRISOR ATERRIMUS.

B. $M$.

Promerops aterrimus? Steph. $\quad$ P. pusillus, $S w . \quad$ P. cyanomelas, Rüpp.? Irrisor aterrimus, G.R.Gr. Rhinopomastes pusillus, $\operatorname{Pr} . B$.

W. and N.-E. Africa.

869. IrRISOR UNICOLOR.

Epimachus unicolor, Licht. Irrisor unicolor, G. R. Gr.

S. Africa.

870. IRRISOR LAMPROLOPHOS.

Epimachus lamprolophos, Wagl. Levaill. Prom. t. 11, 12. Irrisor lamprolophos, G. R. Gr.

Madagascar? E. Africa.

871. IrRISOR INDICUS.

Upupa indica, Lath. Vieill. Ois. dor. Prom. t.9. Promerops cærnleus, Shaw, Nat. Misc. pl.985. Falcinellus cæruleus et F. cyinens, Vieill. Levaill. Prom. t. 7. Epimachus cæruleus, Wagl. Irrisor indicus, G. R. Gr. I. cæruleus, $\operatorname{Pr}, B$.

S. Africa. 
872. Irrisor caudacutus.

Epimachus caudacutus, Vieill. E. obscurus, Wagl. Irrisor caudacutus, G. R. Gr.

Madagascar.

873. IRRISOR SIBILATOR.

Falcinellus sibilator, Vieill. Levaill. Prom. t. 10. Epimachus sibilatrix, Wagl.

S. Africa.

b. Rhinopomastes, A. Smith, 1828.

874. Irrisor Cyanomelas.

B. M.

Falcinellus cyanomelas, Vieill. Levaill. Prom. t. 5,6. Upupa purpurea, Burch. Rhinopomastes Smithii, Jard. Zool. Journ. iv. pl. 1. R. cyanomelas et R. Levaillantii, Less. Promerops purpuratus, $\boldsymbol{S} w$. Epimachus cyanomelas, $\boldsymbol{W a g l}$. Irrisor cyanomelas, G. R. Gr.

S. Africa.

875. IRRISOR MINOR.

B. M.

Epimachus vel Promerops minor, Rüpp. Syst. Uebers. Vög. N. Ost. Afr. t. 8. Rhinopomastes minor, $\operatorname{Pr}$. B. Irrisor minor, G. R. Gr.

N.-E. Africa.

3. Falculla.

Falculia, J. Geoffr. 1836.

876. Falculia palliata.

B. M.

Falculia palliata, J. Geoffr. Mag. de Zool. (1836) Ois. t. 49.

Madagascar.

\section{ii. Eprmachind.}

Epimachinæ, G. R. Gr. 1848; Pr. B. 1849-50.

Upupinæ, p. Pr. B. 1831 ; G. R. Gr. $1840-41$.

Paradiseana, p. Vigors, 1825.

Promeropinæ, G.R. Gr. 1841.

Paradiseinæ, p. Cab. 1847 ; Bl. 1849.

Fregilinæ, p. Pr. B. 1850. 


\section{NEOMORPHA.}

Neomorpha, Gould. 1836.

877. Neomorpha Gouldir.

B. M.

Neomorpha acutirostris et N. crassirostris, Gould, B. of Austr. iv. pl. 19. Neomorpha Gouldii, G. R. Gr. O. Des Murs, Icongr. Ornith. t. 2.

New Zealand.

\section{Eрimachus.}

a. Epimachus, Cuv. 1817.

Cinnamolegus, Less. 1835.

878. Epimachus speciosus.

B. $\mathrm{M}$.

Upupa speciosa et U. striata, Bodd. $\quad P l$. enl. $638,639 . \quad$ U. magna, Gm. U. superba et U. papuensis, Lath. Sonn. Voy. t. 100, 101. Merops fusca, $\mathrm{Gm}$. Promerops superbus et P. striatus, Shav. Falcinellus superbus et F. fuscus, Vieill. Ois. dor. Prom. t. 7. Epimachus magnus, Cuv. E. superbus, Steph. E. speciosus, G. R. Gr. Levaill. Prom. t. 13, 14, 15, Cinnamolegus papuensis, Less. Ois. de Parad. t. 39, 40.

New Guinea.

b. Seleucides, Less. 1835.

Nematophora, G. R. Gr. 1840.

879. Epimachus albus. B. M. Paradisea alba, Blum. P. nigricans, Shaw. P. violacea, Bechst. P. candida, Forst. Levaill. Prom. t. 17. Falcinellus resplendens, Vieill. Gal. des Ois. t. 185. Ois. dor.t. 13. Epimachus albus, Temm. Seleucides acanthylis, Less. Hist. Nat. Parad. t. 36, 37, 38. S. albus et Nematophora alba, $G$. R. Gr.

New Guinea. 
c. Craspedophora, G. R. Gr. 1840.

880. Epimachus magnificus.

B. M.

Falcinellus magnificus, Vieill. Cuv. Règ. Anim. t. 4, f. 2. Levaill. Prom. t. 16. Epimachus splendidus, Steph. E. magnificus, G. R. Gr. Craspedophora magnifica, G.R. Gr. Voy. de la Coqu. Ois. t. 28. Less, Ois. de Parad. t. 32, 33, 34. Lath. Hist. of B. iv. pl. 67*. G. R. Gr. \& Mitch. Gen. of B. pl. 32. (E. paradiseus, by error).

New Guinea and N. Australia (Cape York).

d. Ptilornis, $\$ w_{*} 1825$.

881. Epimachus paradiseus.

B. M.

Ptilornis paradiseus, Sw. Gould, B. of Austr. iv. pl. 100. Epimachus regius, Less. Cent. Zool. t. 3. Voy. de la Cogu. t. 28. Hist. Nat. Parad. t. 29, 30. E. Brisbani, Wils. Ill. of Zool. pl. 9. E. paradiseus, G. R. Gr.

New South Wales.

882. Epimachus Victoria.

B. M.

Ptilornis Victoriæ, Gould, Proc. Zool. Soc. 1849, pl. 12.

N. Australia (Cape York) and Barnard's Isles.

\section{NECTARINID艮.}

Nectarinidæ, Vigors, 1825 ; G: R. Gr. 1840-41; Cab. 1847; Bl. 1849.

Promeropidæ, Vigors, $1825 ;$ G. R. Gr. 1847.

Cinnyridæ, Vigors, 1825; Sw. 1831-37; Selys, 1842.

Trochilidæ, p. Pr. B. 1831.

Cærebidæ, Bl. 1849; Pr. B. 1849-50.

Meliphagidæ, p. $S w .1837$.

Meliphagides, p., Cinnyrides, Dacnides, Epimachides, p. Sundev. 1835.

\section{i. Nectarinine.}

Nectarininæ, G. R. Gr. 1841 ; Cab. 1847 ; Pr. B. 1849-50.

Promeropinæ, G. R. Gr. 1847.

Drepaninæ et Myzomelinæ, p. Cab. 1847.

Upupinæ, p. $\operatorname{Pr}$. B. 1831. 


\section{Drepanis.}

a. Drepanis, Temm. 1820.

Vestiaria, Flem. 1822.

883. Drepanis pactfica.

B. M.

Certhia pacifica, Gm. Levaill. Guêp. t. 19. Vieill. Ois. dor. t. 63. Drepanis pacifica, Temm. Melithreptus pacificus,

Vieill. Vestiaria hoho, Less .

Friendly Islands.

884. Drepanis coccinea.

B. $M$.

Certhia coccinea et C. obscura, Gm. Vieill. Ois. dor. t. 52,53. C. vestiaria, Lath. Gen.Syn. pl, 33, f. 1. Melliphaga coccinea, Merr. Av. Icon. t. iv. Drepanis obscura et D. vestiaria, Temm. D. coccinea, Gr. Melithreptus obscurus et M. vestiarius, Vieill. Hemignathus obscurus, Licht. Berl. Trans. 1838 , t. v. f. 1. Vestiaria evi et V. akaroa, Less. Shaw, Nat. Misc. pl. 75.

Sandwich Islands.

885. ? Drepanis FALCATA.

Certhia falcata, Gm. Drepanis falcata, G.R.Gr.

886. Drepanis sanguinea.

B. M.

Certhia sanguinea et C. virens, Gm. Vieill. Ois. dor.t.66, 67,68 . Nectarinia sanguinea et N. virens, Cuv. N. Byronensis et N. flava, Blox. Griff. An. Kingd. ii. pl. p. 390. Melithreptus virens, Vieill. Furnarius? virens, Steph. Phyllornis tonganensis, Less. Drepanis flava, Gr. D. sanguinea et Myzomela? sanguinea, $G . R . G r$.

Sandwich Islands.

887. Drepanis olivacea.

Merops olivaceus, Shaw. Vieill. Ois.dor. (Prom.) t. 5. Furnarius olivaceus, Steph. Drepanis olivascens, G.R. Gr.

Islands of the South Pacific Ocean? 
b. Hemignathus, Licht. 1838.

Heterorhynchus, Lafr. 1839.

888. Drepanis LUCIDA.

B. $M$.

Nectarinia lucida, Licht. Heterorhynchus olivaceus, Lafr. Mag. de Zool. 1839. Ois. t. 10. Vंestiaria heterorhyncha, Less. Drepanis lucida et D. olivacea, G. R. Gr. Hemignathus lucidus, Licht. Berl. Trans. 1838, I. v. f. 2, 3. H. olivaceus, Licht. Voy. de la Venus. Ois. t. 1.

Sandwich Islands.

c. Moho, Less. 1831.

Acrulocercus, Cab. 1847.

889. Drepanis Nigra.

Merops niger, Gm. M. fasciculatus, Lath. Dixon's Vny. 19. Pl. col. 471. Gracula nobilis, Merr. Av. Icon. t. 2. Epimachus pacificus, Licht. Meliphaga fasciculata, Temm. Philemon fasciculatus, Vieill. Moho niger, G.R. Gr. Acrulocercus niger, $C a b$. Ptiloturus fasciculatıs, Peale.

Sandwich and Triendly Islands.

\section{Nectarinia.}

a. Nectarinia, Illig. 1811.

Cinnyris, Cuv. 1817; Vieill. 1818.

Mellisuga, Vieill. 1816.

890. Nectarinia famosa.

B. M.

Certhia famosa, L. Pl.enl. 83, f, 1. Vieill. Ois. dor. t. 37, 38. Trochilus afer, $L$. Nectarinia famosa, Illig. N. formosa, Pr. B. Cinnyris famosa, Cuv. Levaill. Ois. de l'Afr. t. 289, 290. Shaw, Nat. Misc. pl. 19. Nat. Libr. xvi. pl. 17.

S. and N.-E. Africa.

891. Nectarinia pulchella.

B. M.

Certhia pulchella, L. Pl. enl. 670, f. 1: Vieill. Ois. dor. t. 40. Cinnyris caudatus, Vieill. C. pulchella, Cuv. Nectarinia pulchella, Jard. Levaill. Ois. de l'Afr. t. 293, f. 1. Nat. Libr. xvi. pl. 18. Sw. B. of W. Afr. pl. 14.

S.-W. and N.-E. Africa. 
892. Nectarinia melanopogon.

Nectarinia melanopogon, Licht.

W. Africa (Senegal).

893. Nectarinia cardinalis.

Cinnyris cardinalis, Vieill. Levaill. Ois. de l'Afr. t. 291. Nectarinia cardinalis, G.R.Gr. N. cardinalinus, Jard. Cinnyris violacea, p. Cuv.

S. Africa.

894. Nectahinia violacea.

B. $M$. Certhia violacea, $L . \quad P l$. enl. 670, f. $2 . \quad$ Vieill. Ois. dor. t. 39. C. crocata, Shaw, Nat. Misc. pl. 210. Nectarinia violacea, Illig. Cinnyris violacea, Cuv. C. croceus, Less. Levaill. Ois. de I Afr. t. 292. Nat. Libr. xvi. pl. 16.

S. Africa.

895. Nectarinia platura.

B. M.

Cinnyris platurus, Vieill. Levaill. Vis. de l'Afr. t. 293, f. 2. C.? saccharina, Steph. Nectarinia sylviella, Temm. N. platura, Jard. Nat. Libr. xvi. pl. 19. N. cyannpggos, Licht.? Cinnyris Cuv.

S. and W. Africa.

896. Nectarinia tacazze.

B. M. Certhia tacazze, Stanl. Rüpp. Fauna Abyss. t. 31, f. 3. Nectarinia tacazze, G. R. Gr.

N.-E. Africa.

897. Nectarinia goalpariensis.

B. M. Certhia goalpariensis, Royle, Illustr. of B. pl. 7, f. 1. Lath. Hist. of B. iv. pl. 74. Cinnyris Vigorsii et C. concolor, Sykes. C. miles, Hodgs. C. labecula, Mc'Clell. Nectarinia scheriæ, Tick. N. Lathami et N. goalpariensis, Jard. Nat. Libr. xvi. pl. 27.

India (Goulparah, Nepal).

898. Nectarinia Gouldie.

B. $M$.

Cinnyris Gouldiæ, Vigors. Gould, Cent. of B. pl. 56. Nectarinia Gouldiæ, Jard.

India (Himalayah Mountains). 
899. Nectarinia ignicauda.

B. M.

Cinnyris ignicaudus et C. epimecurus, Hodgs. C. rubricaudata, Bl. Nectarinia phænicura, Jard. Nat. Libr. xvi. pl. 29. N. ignicauda, Hodgs.

India (Nepal, Silhet).

900. Nectarinia NipAlENSIS.

B. M.

Cinnyris nipalensis, Hodgs. Nectarinia nipalensis, Jard. Nat. Libr. xvi. p]. 27.

India (Nepal).

901. Nectarinia Horsfieldit. Nectarinia Horsfieldii, $\boldsymbol{B l}$.

India (Himalaya).

902. Nectarinia siparaja.

Certhia siparaja, Raff. Nectarinia mystacalis, Temm. $\boldsymbol{P l}$. col. 126, f. 3. N. siparaja, Jard. Cinnyris mystacalis, Cuv. C. siparaja, Vig. \&Horsf. Verh. Nat. Gesch. Nederl. ᄂ. 9, f. 1.

Sumatra.

903. Nectarinia saturata. Cinnyris saturata, Hodgs. C. assamensis, $M c^{\prime}$ Clell. Nectarinia Hodgsonis, Jard. Nat. Libr. xvi. pl. 28. N. saturata, $B l$.

India (Nepal).

904. Nectarinia Temmincki.

Nectarinia Temminckii, Müll. Verh. Nat. Gesch. Nederl. t. S, f. 2 .

Sumatra.

905. Nectarinia eximia.

B. M.

Nectarinia eximia, Horsf. N. Kuhlii, Temm. $P l . c o l .376, \mathrm{f}$.

1. Cinnyris Kuhlii, Cuv.

Java. 
b. Cinnyris, $\operatorname{Pr}$. B. 1850.

906. Nectarinia currea.

B. M.

Certhia cuprea et C. rubrofusca, Shaw. Vieill. Ois, dor. t. 23, 27. Cinnyris tricolor et C. nibarus, Vieill. C. cuprea et C. rubrofusca, Cuv. C. erythronotus, Sw. B. of W. Afr. pl. 15. Nectarinia cuprea, Jard.

W. Africa (Cape Coast, Accra, Malemba and Senegal).

907. Nectarinia amethystina.

B. $\mathrm{M}$.

Certhia amethystina, Shaw. Vieill. Ois. dor.t. 5, 6. C. aurifrontalis, Bechst.? Cinnyris auratifrons, Vieill. C. amethystina, Cuv. C. aurifrons, Less. Nectarinia aurifrons, Lichto? N. amethystina, Jard. Nat. Libr. xri. pl. 13. Levaill. Ois. de $l^{\prime}$ Afiv. t. 294, f. 1.1

S. Africa.

908. Nectarinia cyanocephala.

B. M.

Certhia cyanocephala, Gm. Vieill. Ois. dor. t. 7. Cinnyris chloronotus, Sw. B. of W. Afr. pl. 16. C. cyanocephala, Cuv. Nectarinia cyanocephala, Jard. Nat. Libr. xvi. pl. 10.

W. Africa (Malemba, Sierra Leone).

909. Nectarinia collaris.

B. M.

Cinnyris collaris, Vieill. Levaill. Ois. de l'Afr. t. 299. Nectarinia collaris, Jard. Nat. Libr. xvi. pl. 6.

S. Africa and Fernando Po.

910. Nectarinia oruentata.

B. MI.

Nectarinia cruentata, Rüpp. Syst. Uebers. Vög. N.-Ost. Afr. t. 9. N. proteus, Rüpp. Cinnyris cruentata, $\operatorname{Pr}$. B.

N.-E. Africa.

911. Nectarinta fuliginosa.

B. M.

Certhia fuliginosa et C. maculata, Shaw. Vieill. Ois. dor. t. 20, 21. Nectarinia fuliginosa, Jard. Nat. Libr. xvi. pl. 14. Cinnyris fuliginosa et C. maculata, Cuv.

W. Africa (Malemba). 
912. Nectarinia Stangeri. B. M Nectarinia Stangeri, Jard. Illustr. of Orn. n. s. pl. 48. Nat. Libr. xvi. pl. 15. Cinnyris Stangeri, $\operatorname{Pr} . B$.

W. Africa (Niger).

913. Negtarinia rubescens.

Cinnyris rubescens, Vieill. Nectarinia rubẹscens, Jard.

W. Africa (Congo).

914. Nectarinia Metaldica.

B. M.

Nectarinia metallica, Licht. Pl. col. 347, f. 1, 2. Hempr. et Ehrenb. Symb. Phys. t. 1. Rüpp. Zool. Atlas, t. 7. Cinnyris metallica, Cuv.

N.-E. Africa (Abyssinia, Nubia) and Arabia.

915. Nectarinia afFinis.

B. M.

Nectarinia affinis, Rüpp. Fauna Abyss. t. 31, f. 1. Cinnyris affinis, Rüpp.

N.-E. Africa.

916. Nectarinia anea.

Cinnyris æneus, Vieill. Levaill. Ois. de lAfr. t. 297. Nectarinia æneus, Jard.

S. Africa.

917. Nectarinia gularis

Nectarinia gularis, Rüpp. Fauna Abyss. t. 31, f. 2 . Cinnyris gularis, Ruipp.

N.-E. Africa.

918. NeCtarinia RECTIROSTRIS.

Certhia rectirostris, Shaw. Vieill. Ois. dor. t, 75. C. elegans. Vieill. Gal. des Ois. t. 178. Nectarinia rectirostris, Jard. Cinny ris? rectirostris, Steph. C. elegans, Cuv.

Africa or India? 
919. Nectarinia purpurata.

Nectarinia purpurata, Illig. Cinnyris purpurata, $P r . B$. Kittl. Kupf. Vög. t. 28, f. I.

N.-E, Africa.

920. Nectarinia sodi-manga.

Certhia soui-manga, Gm. Briss. Orn. t. 32, f. 2, 3. C. madagascariensis, Lath. Vieill. Ois. dor. t. 18, 19. Nectarinia soui-manga, G. R. Gr. Cinnyris soui-manga, $\operatorname{Pr} . B . \quad$ C. madagascariensis, Cuv.

Madagascar.

921. NeCtarinia virescens.

Cinnyris virescens, Vieill. Ois, dor. t. 34. Nectarinia virescens, $G . R . G r$.

922. NectarinIa splendens.

Cinuyris splendens, Vieill. Ois. dor. t. 2. Nectarinia splendens, G.R. Gr..

W. Africa (Congo).

923. Nectarinia canora.

Certhia canora, Scop. Sonn. Voy. ii. t. 116, f. 2. C. viridis, Lath. C. scarlatina, Sparr. Mus. Carls. t. 58. Nectarinia canora, G.R. Gr. Cinnyris canora, Pr. $B$.

S. Africa.

924. Nectarinia angalanlana.

Certhia angaladiana, Shaw. Vieill. Ois. dor, t. 3,4. C. lotenia, L.? Pl.enl. 575, f. 2, 3. Briss. Orn. t. 33, f. 4, 5. Cinnyris madagascariensis, Quoy \&. Gaim. Voy. de l'Astrol. Ois. t. 5, f. 3. C. angladiana, $P r . B$. C. lotenia, Cuv. Nectarinia angladiana, G. R. Gr.

Madagascar.

925. Nectarinia afra.

B. M.

Certhia afra, L. Edw. B. pl. 347. C. erythrogastra, Shaw, Nat. Misc. pl. 387. Cinnyris smaragdinus et C. pectoralis, Vieill. Levaill. Ois. de l'Afr. t. 300. Vieill. Ois.dor. t. 10. C. caffra, Cuv. Nectarinia afra, Jard. Nat. Libr. xvi. pl. 2.

S. Africa. 
926. Nectarinia chalybea.

B. M. Certhia chalybea, $L . \quad P l$. enl. 246, f. 2 . C. capensis, $L$. Nectarinia chalybea, Temm. Cinnyris chalybea, Cuv. Shaw, Nat. Misc. pl.381. Vieill. Ois. dor. 80, 14? Sw. Zool. Ill. pl. 95. Nat. Libr. xvi. pl. 1.

S. and W. Africa.

927. NeCTARINIA HabYSSINICA.

Nectarinia habyssinica, Hempr. et Ehrenb. Symb. Phys. t. 4. Cinnyris habyssinica, $\operatorname{Pr} . B$.

N.-E. Africa.

928. Nectarinia Verroxi.

B. M.

Cinnyris Verroxi, A. Smith, Ill. S. Afr. Zoal. pl. 57. C. Verreauxi, Pr. B. Nectarinia Verroxi, Jard. Nat. Libr. xvi. pl. 9.

S. Africa.

929. Nectarinia obscura.

B. M.

Nectarinia obscurus, Jard. \& Selby, Ill. of Orn. n. s. pl. 51. Cinnyris obscura, $\operatorname{Pr} . B$.

Fernando Po.

930. Nectarinia olivacea.

Cinnyris olivacea, A. Smith. Nectarinia olivaceus, Jard.

S. Africa (Port Natal).

931. Nectarinia fusca.

Cinnyris fuscus, Vieill. Levaill. Ois. de l'Afr. t. 296. Nectarinia fusca, Jard. Nat. Libr. xvi. pl. 8.

S. Africa.

932. Nectarinia ventsta.

Certhia venusta, Shaw, Nat. Misc. pl. 369 . Cinnyris pusilla, Sw. C. quinticolor, Less. C. venusta, Cuv. Nectarinia renusta, Jard.

W. Africa (Sierra Leone) 
933. Nectarinia pusilla.

Cinnyris pusillus, Vieill. Levaill. Ois. de l'Afr. t. 298. Nectarinia pusilla, Jard.

S. Africa.

934. Nectarinia ohloropygia.

B. M.

Nectarinia chloropygia, Jard. \& Selby, Ill. of Orn. n. s.pl. 50.

Nat. Libr. xvi. pl. 3. Cinnvris chloropygia, Pr. $B$.

Fernando Po.

935. Nectarinia bifasciata.

B. M.

Certhia bifasciata, Shaw. Vieill. Ois. dor. t. 24. Cinnyris nitens, Vieill. C. bifasciata, Pr. B. C. chalybea, p. C $u v$. Nectarinia bifasciata, Jard. Nat. Libr. xvi. pl. 4.

W. Africa (Malemba), Interior of S. Africa.

936. Nectarinta senegalensis.

B. M.

Certhia senegalensis, L. Briss. Orn. iii. t. 34, f. 2. Cinnyris senegalensis, Cuv. C. discolor, Vieill. Ois. dor. t. 8, 9. Nectarinia senegalensis, Jard. Nat. Libr. xvi. pl. 11. Levaill. Ois. de l'Afr. t. 295, f. 2. Kittl. Kupf. Vög. t. 28, f. 2.

S. and W. Africa.

937. Nectarinia soperba.

Certhia superba, Shaw. Cinnyris superbus, Vieill. Ois. dor. t. 22. Nectarinia superbus, Jard.

W. Africa.

938. Nectarinia natalexsis.

B. M.

Nectarinia natalensis, Jard. Nat. Libr. xvi. pl. 12. Cinnyris natalensis, $\operatorname{Pr} . B$.

S.-E. Africa (Port Natal).

939. Nectarinia splendida.

B. M.

Certhia splendida, Shaw, Gen. Zool.pl. 26. Vieill. Ois. dor. t. 82. Levaill. Ois. de l'Afr. t. 295, f. 1 . Cinnyris bombycinus, Vieill. C. splendida, Cuv. C. lucidus et C. sugnimbindus, Less. Nectarinia splendida, Jard. Nat. Libr. xri. pl. 5 .

S. and W. Africa. 
940. Nectarinia Adelberti.

B. M.

Cinuyris Adelberti, Gerv. Mag. de Zool. 1833, t.2. C. Ebuensis. Thoms. MSS. Nectarinia Adelberti, Jard. \& Selby, Ill. of Orn.n.s. pl. 49. Nat. Libr. xvi. pl. 30.

W. Africa (Senegal and Niger).

941. ? Nectarinia verticalis.

Certhia verticalis, Lath. Vieill. Ois. dor. t. 25? Nectarinia verticalis, G. R. Gr.

Africa.

942. ? Nectarinia aurantia.

Certiia aurantia, $G m$. Nectarinia aurantia, G. R. Gr. Africa.

943. ? Nectarinia frontalis.

Certhia frontalis, Lath. Nectarinia frontalis, G. R. Gr.

Africa.

944. ? NeCTARINIA COCCINIGASTRA.

Certhia coccinigastra, Lath. Nectarinia coccinigaster, $G$. R. Gr.

Africa.

945. ? NeCTARINIA TABACINA.

Certhia tabacina, Lath. Nectarinia tabacina, G. R. Gr.

Africa?

946. ? Nectarinia ERYTHROTHORAX.

Cinnyris erythrothorax, Vieill. Nectarinia erytbrothorax, $G$. R. Gr.

W. Africa (Angola).

947. ? Nectarinia Perreini.

Cinnyris Perreini, Vieill. Nectarinia Perreini, Hartl.

W. Africa (Congo). 
948. Nectarinta Mahrattensis.

B. M Certhia asiatica, C. mahrattensis, C. chrysoptera, C. currucaria et C. cirrhata, Lath. Edw. B. pl. 265. Vieill. Ois, dor. t. 12. Cinnyris cirrhatus, Vieill. C. orientalis, Frankl. C. currucaria, Cuv. C. epauletta et C. strigula, Hodgs. C. iodeus, Less. C. asiatica, $\operatorname{Pr} . B$. Nectarinia mahrattensis, Jard. Nat. Libr. xvi. pl. 24. N. asiatica, Bl.

India and Malayan Peninsula.

949. Nectarinia simplex.

Nectarinia simplex, Miill. Verh. Nat. Gesch. Nederl. t. 8, f. 4. N. frontalis, $B l$. Cinnyris simplex, $\operatorname{Pr} . B$.

Sumatra and Borneo.

950. Nectarinia lotenia.

B. M.

Certhia lotenia, $L . E d w . B$. pl. 265, upp. fig. C. polita, Sparr. Mus. Carls. t. 59. C. purpurata, Shaw. Vieill. Ois. dor. t. 11. Cinnyris purpurata, Cuv. C. lotenia, Pr. B. C. orientalis, Frankl. Nectarinia lotenia, Jard. Nat. Libr. pl. 23.

India; Malayan Peninsula and Ceylon.

951. Nectarinia cingalensis.

Certhia cingalensis, Lath. Nectarinia phænicotis, Temm. Pl. $c_{0} l .108$, f. 1, 338, f. 2. N. cingalensis, G. R. Gr. Cinnyris phænicotis, $\mathrm{Pr}, \mathrm{B}$.

Malayan Peninsula and Archipelago.

952. NeCTARINIA FRENATA.

Nectarinia frenata, Müll. Verh. Nat. Gesch. Nederl. 1. 8, f. 1. Cinnyris frenata, $\operatorname{Pr} . B$.

Celebes and New Gninea.

953. Nectarixia australis.

B. M.

Nectarinia australis, Gould, N. pectoralis, Macgill.

N. Australia (Cape York).

954. Nectarixia solaris.

Nectarinia solaris, Temm. Pl. col. 347, f. 3. Verh. Nat.

Gesch. Nederl.t. 8, f. 5. Cinnyris solaris, Cuv.

Amboina and Timor. 
955. Nectaminia pectoralis.

B. M.

Nectarinia pectoralis, Horsf. N. eximia, Temm. Pl. col. 138, f. 1. Miull. Verh. Nat. Gesch. Nederl. t. 9, f. 2. Nat. Libr. xvi. pl. 26, f. 1. Cinnyris eximia, Cuv. C. pectoralis, Steph. Pl. enl. 576, f. 4. Pr. B. Briss. Orn. iii. t. 34, f. 4 .

Malayan Archipelago (Java).

956. Nectarinia Macklotii.

Nectarinia pectoralis, p. Temm. Pl. col. 138, f. 2, 3. Müll. Verh. Nat. Gesch. Nederl, t. 9. Cinnyris Macklotii, Pr. B.

Malayan Archipelago.

957. Nectarinia Flam maXillaris.

Nectarinia flammaxillaris et N. jugularis, $B l$. Cinnyris flammaxillaris, Pr. $B$.

Malayan Peninsula.

958. Nectarinia zeylonica.

B. M. Certhia zeylonica, L. Sonn. Voy. t. 30, f. A. C. quadricolor, Scop. C. dubia, Shaw Vieill. Ois. dor. t. 16, 17. Cinnyris sola, Jerd. C. lepida, Sykes. C. nigralbus, Less. C. zeylonica, Cuv. Nectarinia philippensis, Meyen. N. zeylonica, Jard. Nat. Libr. xvi. pl. 20.

Ceylon, India and Malayan Peninsula

959. Nectarinia Minima.

Cinnyris minima, Sykes. Nectarinia minima, Jard. Nat. Libr. xvi. pl.

India (Deccan, Malabar).

960. Nectarinia Hasseltir.

Certhia sperata, Raff. C. brasiliensis, Shaw. Nectarinia Hasseltii, Temm. Pl. col. 376, f. 3. N. Phayrei, Bl. Verh. Nat. Gesch. Nederl. t. 10, f. 5. Cinnyris Hasseltii, Cuv. Nat. Libr. xvi. pl. 22.

Malayan Peninsula and Archipelago. 
961. Nectarinia affinis. Certhia affinis, Shaw. C. sperata, L.? C. chalybea, Scop. Sonn. Voy. t. 30, f. C. Vieill, Ois. dor. t. 32, 33. Cinnyris sperata, Cuv.? C. coccinogaster, Cuv. Nectarinia affinis, Jard. Nat. Libr. xvi. pl. 21. N. coccinigastra, Temm. Pl. col. 388 , f. 3.

Philippine Islands.

962. Nectarinia Jugularis.

B. M.

Certhia jugularis, L. Pl. enl. 576, f. 3? Briss. Orn. iii. 1. 32, f. 5. Vieill. Ois. dor. t. 29, 30. Nectarinia jugularis, Jard. Nat. Libr. xvi. pl. 25, f. 2 .

Philippine Islands.

963. Nectarinia calcostethia.

Nectarinia calcostethia, Jard.

East India Islands.

964. Nectarinta aspasia.

Cinnyris aspasia, Less. Voy. de la Coqu. t. 30, f.4. Nectarinia aspasia, $G . R$. Gr.

Celebes, Amboina and New Guinea.

965. Nectarinia zenobia.

Cinnyris zenobia, Less. Voy. de la Coqu. t. 30, f. 3. Nectarinia zenobia, G. R. Gr.

New Guinea.

966. Nectarinia Eques.

Cinnyris eques, Less. Voy. de la Coqu. t. 31, f. 1. Nectarinia eques, $G$. $R$. $G r$.

Island of Waigou; New Guinea.

967. ? NeCTARINIA MACASSARIENSIS.

Certhia macassariensis, $G m$. Seba, Thes, i. t. 63, f. 3 . Nectarinia? macassariensis, G. R. Gr.

Islands of Bally and Macassar. 
968. ? Nectarinia MaNillensis.

Certhia manillensis, $G m$. Nectarinia manillensis, G. R. Gr. Philippine Islands.

969. ? Nectarinia AMboinensis.

Certhia amboinensis, Gm. Seba, Thes. t. 26, f. 2. Nectarinia amboinensis, G. R. Gr.

Amboina.

970. ?? Nectarinia oMnicolor.

Certhia omnicolor, Gm. Seba, Thes. t. 69, f. 5. Cinnyris omnicolor, Cuv. Nectarinia omnicolor, G. R. Gr.

Ceylon.

971. ? Nectarinia Parietum.

Certhia parietum, Lath. Sylvia indica, Vieill. Nectarinia parietum, G. R. Gr.

India.

972. ? Nectarinia sola.

Cinnyris sola, Vieill. Nectarinia sola, G. R. Gr.

India (Pondicherry).

973. ? Nectarinia subflava.

Cinnyris subflavus, Vieill. Nectarinia subflava, G. R. Gr. India.

974. ? NectaRinia indica.

Certhia indica, Gm. Seba, Thes. t. 19, f. 2. Nectarinia indica, G. R. Gr.

India.

975. ? Nectarinia violaceofrons.

Cinnyris violaceofrons, Vieill. Nectarinia violaceofrons, $G$. R. Gr. 
976. ? Nectarinia RUFicolis.

Cinnyris ruficollis, Vieill. Nectarinia ruficollis, G. R. Gr. ?

977. ? Nectarinia CINEREICOLLIS.

Cinnyris cinereicollis, Vieill. Nectarinia cinereicollis, G. R. $G r$.

978. Nectarinia nigrescens.

Cinnyris nigrescens, Vieill. Nectarinia nigrescens, G. R. Gr.

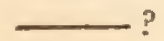

979. ? NeCtarinia Nigrogaster.

Cinnyris nigrogaster, Vieill. Nectarinia nigrogaster, $G, R$. Gr.

980. ? Nectarinia ledcogaster.

Cinnyris leucogaster, Vieill. Nectarinia leucogaster, $G . R$. $G r$.

Timor.

c. Promerops, $\mathrm{Br} .1760$.

Falcinellus, Vieill. 1816.

Ptiloturus, Sw. 1837.

Ptilurus, Strickl. 1841.

981. Nectarinja promerops.

B. $\mathbf{M}$.

Upupa promerops et Merops cafer, L. Pl. enl.637. Levaill. Ois. de l'Afr. t. 287, 288. Nectarinia promerops, Temm. N. caffra, Licht. Cinnyris longicaudatus, Vieill. Promerops cafer, Shaw. P. capensis, Less. Ptiloturus capensis, Sw. P. cafer, G.R.Gr. Vieill. Ois. dor. t. 4.

S. Africa. 
c. Anthreptes, $S w$. 1831 .

982. Nectarinia malaccensis. B. M. Certhia malaccensis, Scop. Sonn. Voy. t. 116, f. 1. C. lepida, Sparr. Mus. Carls. t. 35. Nectarinia javanica, Horsf. $\mathbf{N}$. malaccensis, G. R. Gr. Cinnyris javanica, Cuv. C. lepidus, Vieill. Gal. des Ois. t. 177. Anthreptes javanica, Sw. Zool. Illustr. pl. 121. Pl. col. 126, f. 1,2. A. lepida, $\operatorname{Pr} . B$.

Malayan Archipelago.

983. NeCtarinia hypogrammica.

B. MI.

Nectarinia hypogrammica, Mül. Verh. Nat. Gesch. Nederl. t. 8, f. 3. N. nuchalis, $B l$. Anthreptes macularia, $B l$. A. hypogrammica, $\mathrm{Pr} . \mathrm{B}$.

Malayan Archipelago.

984. Nectarinia Fraseri.

B. M.

Nectarinia Fraseri, Jard. \& Selby, Ill. of Orn. n. s. pl. 53. Anthreptes Fraseri, $P$ r. $B$.

W. Africa.

985. Nectarixia Longuemari.

B. M.

Cinnyris Longuemari, Less. Ill. de Zool. t. 23. Anthreptes leucosoma, Sw. B. of W. Afr. pl. 17. A. Longuemari, Hartl. Nectarinia Longuemari, G. R. Gr.

W. Africa.

986. Nectarinia melasona.

Cinnyris melasoma, Less. Nectarinia melasoma, G. R. Gr. Anthreptes melasoma, $\operatorname{Pr} . B$.

W. Africa.

\section{d. Arachnotheres, Temm. 182 ?}

987. Nectarinia Longirostris.

B. M.

Certhia longirostris, Lath. Nectarinia et Arachnothera longirostris, Temm. Pl. col. 84, f. 1. Cinnyris longirostris, Griff. An. Kingd. pl. p. 391.

Malayan Archipelago. 
988. Nectarinia inornata.

Cinnyris longirostris, Jerd. Arachnothera inornata aut A. affinis, $B l$. A. longirostris, $\operatorname{Pr} . B$.

Malayan Peninsula.

989. Nectarinia affinis.

B. M.

Nectarinia affinis, Horsf. $\quad$ N. inornata, Temm. Pl. col. 84, f.

2. Arachnothera inornata, Temm. A. aftinis, G. R. Gr.

Malayan Archipelago.

990. Nectarinia Chrysogenys.

B. MI.

Certhia longirostra, Raff. Nectarinia et Arachnothera chrysogenys, Temm. Pl. col. 388, f. 1 . A. flavigenis, $\$ w$.

Malayan Archipelago.

991. Nectarinia magna.

B. M.

Cinnyris magna, Hodgs. Arachnothera inornata, Mc'Clell.

A. magna et A. chrysopus, Hodgs.

India (Nepal).

992. Nectarinia modesta.

Anthreptes modesta, Eyton. Arachnothera latirostris, Bl. A. modesta, $\operatorname{Pr} . \boldsymbol{B}$.

Malayan Peninsula.

993. Nectarinia flavigaster.

Anthreptes flavigaster, Eyton. Arachnothera flavigaster, $G$. R. $G r$.

Malayan Peninsula.

994. Nectarinia robusta.

Arachnothera robusta, Miill. \&. Schl. Verh. Nat. Gesch. Nederl. t. 11, f. 1 .

Sumatra.

995. Nectarinia armata.

Arachnothera armata, Mill. \&. Schl. Verh. Nat. Gesch. Nederl. t. 11, f. 2.

Java and Sumatra. 
996. Nectarinia nove gUinex.

Cinnyris novæ guineæ, Less. Arachnothera noræ guineæ, Mül. \& Schl. Verh. Nat. Gesch. Nederl. t. 11, f. 3.

New Guinea.

997. Nectarinia uropygialis.

B. M. Arachnothera uropygialis, G. R. Gr. \&itch. Gen. of B. pl. 33.

Malayan Archipelago.

\section{Dickum.}

a. Dicæum, Cuv. 1817.

Myzanthe, Hodgs. 1843.

998. DiCAUM HIRUNDINACEUM.

B. M.

Sylvia hirundinacea, Shaw. Lewin, N.H. Birds. pl.7. S. rubricollis et Pipra gularis, Lath. Mag. de Zool. 1833. Ois. t. 14. Pardalotus gularis, Temm. Pipra Desmaresti, Leach. Zool. Misc. pl. 41. Dicæum pardalotus et D. hirundinaceum, Cuv. D. atrogaster, Less. D. sanguineum, Sw. Myzanthe hirundinacea, Gould, B. of Austr. ii. pl. 34. Shav, Nat. Misc.pl. 19.

New South Wales; S. and W.Australia.

999. Diceum IGNIPECTUS.

B. II.

Myzanthe (Micrura) ignipectus, Hodgs. M. ignipectus, $B l$. Dicæum ignipectus, $G$. $R$. $G r$.

India (Nepal).

1000. Dic eum cruentatum.

B. M.

Certhia cruentata, L. Edw. B. pl. 81. C. coccinea, Scop. Sonn. Voy. t. 117, f. 1. Vieill. Ois. dor. t. 35. C. erythronota, Lath. Dicæum rubricapillum, Less. D. coccineum, $G$. R. $G r$. D. cruentatum, $B l$. D. erythronotum, $C u x$. Nectarinia ignita, Begbie.

India. Malayan Peninsula and Archipelago. 
1001. Diceum trigonostigma.

B. M.

Certhia trigonostigma, Scop. Sonn. Voy. t. 117, f. 2. C. cantillans, Lath. $\quad P l$. col. 478, f.3. Dicæum croceiventre, Vigors. D. trigonostigma, G. R. Gr. D. cantillans, Cuv.

Malayan Peninsula and Archipelago.

1002. DICAUM CHRTSORRHÆUM.

Dicæum chrysorrhæum, Temm. $P l . c o l .478$, f. 1. D. chrysochlorum, $B$.

Malayan Peninsula and Archipelago.

1003. Dic auM CONCOLOR.

B. M.

Dicæum concolor, Jerd. Ill. Ind. Orn. pl. 39.

India (Madras).

1004. Dic कuM ERTTHRORHYNCHOS.

Certhia erythrorhyncha, Lath. Nectarinia minima, Tick. Dicæum Tickellii et D. minimum, $B l$. D. concolor, p. et Nectarinia? erythrorbyncha, G. R. Gr. Myzanthe inornata, Hodgs.

India.

1005. DiC EUM CELEBICUM.

Dicæum celebicum, Müll. D. Leclancherii, Lafr.

Celebes.

1006. Dicanu Mackloti.

Dicæum Macklotii, Miill.

Timor.

1007. Dic Eum SANGUINolentum.

Dicæum sanguinolentum, Temm. Pl. col. 478, f. 2. Java.

1008. DiC EUM PAPUENSE.

B. M.

Pipra papuensis, Gm. Pl. enl. 707, f. 2. Dicæum paptense, G. R. Gr. D. rubriventer, Less.

New Guinea. 
1009. Dicf.um PeCtorale.

Dicæum pectorale, Müll.

New Guinea.

1010. Dickum erythrothorax.

Dicæum erythrothorax, Less. Voy. de la Coqu. t. 30, f. 1, 2.

Amboina.

1011. Dickum Rubescens.

B. M.

Dicæum cruentatum, Horsf. D. rubescens, Vieill. D. erythropygia, p. Cuv. Levaill. Ois. de l Afr. t. 136. D. rubrucana, Pr. B. Nectarinia rubrocana, Temm. Pl. col. 108, f. 2, 3.

Malayau Archipelago.

1012. Dic 玉um Rubrum.

Certhia rubra, Gm. Vieill. Ois. dor. t. 54. C. cardinalis, p. Shaw. Dicæum atripes, Vieill, D. scarlatinum, Schinz. D. rubrum, G. R. Gr.

Island of the South Seas.

1013. Dic 玉um griseum.

Certhia grisea, Scop. Sonn. Voy. t. 117, f. 3. C. tæniata, Shaw. Dicæum flariceps, Vieill. D. griseum, G.R.Gr. D. tæniatum, Cuv.

China?

1014. Diceum Pygmade.

Nectarinia pygmæa, Kittl. Mem. de St. Petersb. 1835, t. 2.

Philippine Islands.

1015. DiC EUM CINEREUM.

Dicæum cinereum, Kittl. Kupf. Vög. t. 8, f. 2. Drepanis cinerea, Kittl. Mem. de St. Petersb. 1835, t. 5.

Philippine Islands.

1016. Dicedu Maugei.

Dicæum Maugei, Less.

Timor. 
1017. Dickum Aterrimum.

Dicæum aterrimum, Less. ?

1018. Dic瓜UM NIGRUM.

Dicæum niger, Less. Cent. de Zool. t. 27.

New Guinea.

1019. Dicaum saccharinum.

Certhia saccharina, Lath. Dicæum saccharinum, Eyton. Malacca.

1020. Dickum? RUfum.

B. M

Nectarinia rufa, Blox. Voy. of Sulphur, pl. Drepanis rufa, G. R. Gr.

Sandwich Islands.

1021. DICEUM

Dicæum

Voy. au Pole Sud, Ois. t. 22, f. 4.

b. Prionochilus, Strickl. 1841.

Triglyphidea, Reichenb. 1850.

1022. DíAUM OBSOLETUM.

B. M.

Pardalotus obsoletıs, Miill. \&. Schl. Dicæum obsoletum, G. R. Gr.

Timor.

1023. Dicaum Percussum.

B. M

Pardalotus percussus, Temm. Pl. col. 394, f. 2. Dicæum ignicapillum, Eyton. D. percussum, G. R. Gr. Prionochilus percussus, Strickl.

Malayan Archipelago.

1024. Dic Eum THORACICUM.

Pardalotus thoracicus, Temm. Borneo. 
1025. Dic eum maculatum.

Pardalotus maculatus, Temm. $P l . c o l .600$, f. $3 . \quad$ Prionochilus thoracicus, p. $\operatorname{Pr}$. B. Dicæum maculatum, G. R. Gr. Borneo.

c. Pachyglossus, Hodgs. 1843.

1026. Dicaum melanoxanthum.

Pachyglossus melanoxanthus, Hodgs. Dicæum melanoxanthum, G. R. Gr.

India (Nepal).

\section{d. Piprisoma, $B l .1844$. Parisoma, Jerd.}

1027. Dic

B. M.

Fringilla agilis, Tick. Pipra squalida, Burt. Psarisoma vireoïdes, Jerd. Piprisoma agile, $\boldsymbol{B l}$. Dicæum agile, $G$. R. $G r$.

India (Himalayah Mountains).

ii. Cerebine.

Cærebinæ, G. R. Gr. 1840-41-46; Pr. B. 1849-50.

Dacnidinæ, Cab. 1847.

\section{Cæreba.}

Cæreba, Vieill. 1816.

Nectarinia, Cuv. 1817.

Arbelorhina, Cab. 1847.

1028. Cereba ctanea.

B. M.

Certhia cyanea, $L$. Pl. enl. 83, f. 2. Vieill. Ois. dor. t. 4I, 42, 43. C. cayana, $L . \quad P l . e n l .682$, f. $2 . \quad$ C. flavipes, Gm. C. cyanogastra, Lath. C. armillata, Sparrm. Mus. Carls. t. 36. Cæreba cyanea, Vieill. Nectarinia cyanea, Cuv. Arbelorhina cyanea, $\dot{C} a b$.

S. America (Guiana, Brazil, Bolivia). 
1029. C.erebi c frulea.

B. M.

Certhia cærulea, L. Edw. B. pl. 21, f. 1. Sparr. Mus. Carls. t. 82. Vieill. Ois. dor. t. 44, 45. C. ochrochlora, Gm. Penn. Gen. of B. pl. 3, f. 2. C. surinamensis, Lath. Fringilla cyanomelas, $G m$. Nov. Comm. Ptr. xi. t. 15, f. 6. Cæreba cærulea, Vieill. Nectarinia cærulea, Cuv. Arbelorhina cærulea, $C^{\prime} a b$.

S. America (Brazil).

1030. Careba gutturalis.

B. M.

Certhia gutturalis, $L . \quad P l$. enl. 578, f. 3. Cæreba gutturalis, G. R. Gr. Cinnyris gutturalis, Cuv.

S. America (Brazil).

1031. Careba gularis.

Certhia gularis, Sparr. Mus. Carls. t. 79. Cæreba gularis, G. R. Gr.

Martinico.

1032. C.ereba trochilea.

Certhia trochilea, Sparr. Mus. Carls. t. 80. Cæreba trochilea, G. R. Gr.

S. America.

1033. CAREB A Nitida.

Cæreba nitida, Hartl.

S. America.

1034. ? CEREBA COCCINEA.

Certhia coccinea, $L$. C. mexicana, Gm. Seba, Thes. t. 70, f.

8. Cæreba coccinea, G. R. Gr.

Central America (Mexico).

1035. ? C \&REBA Fulva.

Certhia fulva, Gm. Cæreba fulva, G. R. Gr.

S. America.

1036. ? C.ereba purpurea.

Certhia purpurea, Gm. Seba, Thes. t. 72, f. 2. Cæreba purpurea, G. R. Gr.

N. America (Virginia). 
1037. ? Cereba variegata.

Certhia variegata, Gm. Seba. Thes. t. 3, f. 3 . Cæreba variegata, G. $R$. $G$ r.

S. America.

\section{DaCNIS.}

a. Dacnis, Cuv. 1817.

1038. Dacnis cayana.

B. M.

Certhia cayana, L. Pl. enl. 669, f. 1,2. Vieill. Gal. des Ois.

t. 165. Dacnis cayana, Cuv.

S. America (Guiana, Bolivia).

\section{DaCNis CYanocephala.}

Certhia cyanocephala, L. Dacnis cyanocephala, G. R. Gr. Sw. Zool. Illustr. pl. 117. D. cyanater, Less.

S. America (Bolivia, Peru).

1040. DaCnis angelica.

Dacnis angelica, De Filippi, Pr. B. Consp. p. 490.

S. America.

1041. DaCNis PLUMBEa.

Sylvia plumbea, Lath. Dacnis plumbea, Cab. Sylvia cæru-

lescens, Pr.Max.? Minotilla cærulescens. G. R. Gr.??

S. America (Brazil, Peru).

1042. DaCnis flaviventer.

Dacnis flaviventer, D'Orb. et Lafr. Voy. dans l'Amer. Mer. Ois. t. 13, f. 2.

S. America (Bolivia).

1043. Dacnis Bicolor.

Dacnis bicolor, Beckl. Mem. de Mosc. vii, t. 23.

S. America (Guiana). 
1044. Dacnis B Rasiliensis.

Certhia brasiliana, $G m$. Briss. Orn, iii. t. 32, f. 4. Dacnis brasiliana, G. R. Gr.

S. America (Brazil).

1045. Dacnis spiza.

Certhia spiza, $L$. Seba, Thes. ii. t. 3, f. 4. Pl. enl.578, f. 1, 682, f. 2. Dacnis spiza, G. R. Gr.

S. America (Brazil, Cayenne).

1046. DACNis ATRICAPILla.

Cæreba atricapilla, Vieill. C. spiza, var. $\beta$., Lath. Edw. B. pl. 348. Pl. enl. 578. f. 2, et 682. f. 1. Vieill. Ois. dor. 1. 49. Merops spiza, Merr. Icon. Av. t. 5. Nectarinia mitrata, Licht. Dacnis atricapilla, G. R. Gr.

S. America (Guiana, Brazil, Bolivia).

1047. Dacnis ANALis.

Dacuis analis, D'Orb. \&. Lafr.

S. America (Bolivia).

1048. Dacnis Rufocinerea.

Dacnis rufo-cinerza, $\operatorname{Pr}$. B. Consp. p. 401.

S. America (Bolivia).

\section{b. Conirostrum, D'Orb. \& Lafr.}

1049. DACNIS CINEREA.

Conirostrum cinereum, D'Orb. \& Lafr. Voy. dans $l$ Amer. Mer. Ois. t. 59 , f. 2.

S. America (Bolivia, Peru).

1050. DaCNis AlBifrons.

B. M.

Conirostrum albifrous, Lafr. Mag. de Zool. 1843, Ois. t. 35.

S. America (Columbia).

1051. DaCnis AtrocyaneA.

Conirostrum atrocyaneum, Lafr.

S. America (Columbia). 
1052. DaCNis SitTicolor. Conirostrum sitticolor, Lafr. C. bicolor, Less. G. R, Gr. \& Mitch. Gen. of B. pl. 34 .

S. America.

1053. DACNis sUPERCILIOSA.

Conirostrum superciliosum, Hartl.

S. America.

1054. DaCnis Caruleifrons.

Conirostrum cæruleifrons, Lafr.

B. M.

S. America.

c. Certbiola, Sundev. 1835.

105̃. Dacnis flateola.

B. $M$.

Certhia flaveola, $L . \quad E d w . B$. pl. 122, et pl. 362, f. 2. C. bartholemica, Sparr. Mus. Carls. t. 57. Sw. Zool. Illustr. pl. 52. Nectarinia antillensis, Less. Gosse, B. of Jam. pl. . N. flaveola, Cuv. Furnarius flaveolus, Steph. Vieill. Ois. dor. t. 51.

W. Indies and S. America.

\section{TROCHILID无.}

Trochilidx, Vigors, 1825; Sw. 1827-31-37; Pr. B. 1831-38 $-49-50$; G.R. Gr. 1840-41-44; Cab. 1847 ; Bl. 1849. Trochili, Sundev. 1835.

i. Trọchilinæ.

Trochilinæ, G. R. Gr. 1840-41-48; Pr. B. 1849-50.

Lamporninæ, G. R. Gr. 1840-41.

Lampornithinæ, $P r, B .1849$.

Grypinæ, G. R. Gr. 1848.

Phœthorninæ, G. R. Gr. 1840-41.

Phæthornithinæ, Pr. B. 1849.

Mellisuginæ, G, R. Gr. 1848. 


\section{T'rochilus.}

Trochilus, $I .1748$.

Troglodytes, $L$. 1735 .

a. Phœthornis, Sw. 1847.

Phœtornis, Less. 1829.

Phæthornis, Jard. 1833.

Phaëthornis, Strickl. 1841.

1056. Trochilus superciliosus.

B. $\mathrm{M}$

Trochilus superciliosus, $L . \quad P l . e n l .600$, f. 3. Vieill. Ois. dor. t. 17, 18, 19. Less. Col. t. 6, 7. Nat. Libr. xv. pl. 26, 27. Tr. malaris, Nordm.? Phœthornis superciliusus, Sw. P. malaris, G. R. Gr.? Trochilus (Phœtornis) superciliosus, Less. Polytmus superciliosus, Boie.

South America (Guiana, Brazil, Bolivia).

1057. Trochilus melanotis.

Trochilis melanotis, Nordm. Phætornis melanotis, G.R. Gr. S. America.

1058. Trochilus LEUCOPHRYS.

Trochilus leucophrys, Nordm. Phætornis leucophrys, G. R. Gr.

S. America.

1059. Trochilus GuY.

Trochilus Guy, Less. Troch. t. 44. Trochilus (Phœtomis) Guy, Less. Phæthornis Guy, Jard.

S. America (New Granada).

1060. Trochilus emilia.

Trochilus emiliæ, Bourc. Phætornis emiliæ, G. R. Gr.

S. America (Sta. Fè de Bogota).

1061. Trochilus EURYNonus.

B. M.

Trochilus eurynomus, Less. Troch. t. 31. Phæthornis eurynomus, Jard. Nat. Libr. xr. pl. 28. Gould, Monogr. Troch. pl. 5. P. eurynome, $\operatorname{Pr} . B$. Trochilus (Phœtornis) eurynomus, Less.

S. America (Brazil). 
1061. Trochilus Pretrei.

B. M.

Trochilus Pretrei, De Lattr. \& Less. Phætornis Pretrei, G. $R$. Gr. \&. Mitch. Gen. of B. pl. 35.

S. America (Brazil).

1062. Trochilus Bourcieri.

Trochilus Bourcieri, Less. Troch. t. 18. Trochilus (Phcetornis) Bourcieri, Less. 'Phæthornis Bourcieri, Jard.

S. America (Brazil).

1063. Trochiles hispidus.

Trochilus (- ?) hispidus, Gould. Phætornis hispidus, G. R. Gr.

\section{S. America (Peru?).}

1064. Trochilus apicalis.

Trochilus apicalis, Licht. Phætornis apicalis, G. R. Gr. Trochilus (Polytmus) apicalis, Tschudi.

S. America (Рeru).

1065. Trochillis Philippi.

Trochilus Philippi, Bourc. Phætornis Philippii, G. R. Gr.

S. America.

1066. Trochilus Augustus.

Trochilus Augustus, Bourc. Phætornis Augusta, G. R. Gr.

S. America (Venezuela).

1067. Trochilus Lovguemareus.

B. M.

Trocbilus Longuemareus, Less. Troch. t. 2 and 62. Trochilus (Phœtornis) Longuemareus, less. Phæthornis Longuemareus, Jard. Trochilus leucophrys, Nordm. Phætornis leucophrys, G. R. Gr.

S. America (Guiana).

1068. Trochilus IntermeduUs.

B. M.

Trochilus intermedius, Less. Troch. t. 19. Trochilus (Phœtornis) intermedius, Less. Phæthornis intermedius, Jard.

S. America (Brazil). 
1069. Trochilus BRasiliensis.

Trochilus squalidus, Natt. Pl. col. 120, f. 1. Less. Col.t. 8. Phætornis brasiliensis (Briss.), G.R.Gr.

S. America (Brazil, Bolivia).

1070. Trochilus eremita.

'Trochilus eremita, Gould, Monogr. Troch. t. 6. T. brasiliensis, Temm. Pl. col. 120, f. 2. Phaëthornis eremita, $\operatorname{Pr}$. B.

S. America (Brazil).

1071. Trochilus PYGM बUS. B. M. Trochilus pygmæus, Spix, Av. Bras. t. 80, f. 1. T. Davidianus, Less. Troch. t. 13 ? T. rufigaster, Less. Col.t. 9. Trochilus (Phœtornis) rufigaster et T. (P.) Davidianus? Less. Phætornis rufigaster, G. R. Gr. P. Davidianus, Jard.P Trochilus (Polytmus) pygmæus, Tschudi.

S. America (Guiana).

1072. Trochimus $Y_{\text {ARUQui. }}$

Trochilus Yaruqui, Bourc.

S. America (Ecuador).

1073. ?'Trochilus conurus.

Trochilus paroninus $(M S S$.) et Wedge-tailed Hummingbird, Lath. Hist. T. conurus, Steph. Phætornis conurus, G. R. Gr.

S. America.

1074. ? Trochilus clliatus.

Trochilus ciliatus (MSS.) et Buff-striped Humming-bird, Lath. Hist. Phætornis ciliatus, G.R. Gr.

S. America.

1075. ? Trochilus Maxillosus.

Trochilus maxillosus, Nordm. Phaëthornis maxillosus, Pr. B. S. America. 
b. Oreotrochilus, Gould, 1847.

1076. Trochilus ESTELLA.

B. M.

Trochilus estella, D'Orb. \& Lafr. Voy. dans l'Amer. Mer. Ois. t. 61, f. 1. Ornismya Ceciliæ, Less. Oreotrochilus estella, Gould, Monogr. Troc. pl. 2.

S. America (Bolivia).

1077. Trochilus LEUCOPLEURUS. B. M. Trochilus leucopleurus et Oreotrochilus leucopleurus, Gould, Monogr. Troch. pl. 3.

S. America (Chilian Cordilleras).

1078. Trochilus chimborazo.

Trochilus chimborazo, Bourc. Oreotrochilus chimborazo, Gould.

S. America (Ecuador).

1079. Trochilus adela.

Trochilus adela, D'Orb. \& Lafr. Voy. dans l'Amer. Mer. Ois. t. 61, f. 2. Oreotrochilus adela, Gould, Monogr. Troch. pl. 4 .

S. America (Bolivia).

1080. Trochilos me lanogaster.

Oreotrochilus melanogaster, Gould.

S. America (—?)

1081. Trochilus pichincha.

B. $M$.

Trochilus pichincha, Bourc. Oreotrochilus Jamesoni, Jard. Contr. Ornith. 1850, pl. O. pichincha, Pr. B.

S. America (Ecuador). 
c. Grypus, Spix, 1824.

Ramphodon, Less. 1829.

Phœthornis, p. Sw. 1828.

1082. Trochilus NaviUs.

B. M.

Trochilus nævius, Dum. T. squamosus, Licht. Pl.col. 120, f. 3. Orthorhynchus squamosus, Cuv. Grypus ruficollis, Spix, Av. Bras. t. 80, f. 3. G. nævius, G. R. Gr. Trochilus (Ramphodon) maculatus, Less. Ramphodon maculatum, Less. Col. t. 1. Nat. Libr. xv. pl. 1. Mellisuga? nævia, Steph.

S. America (Brazil).

\section{d. Eutoxeros, Reichenb. 1849.}

Glaucis, p. $\operatorname{Pr} . B .1850$.

1083. Trochilus Aquila.

Trochilus aquila, Lodd. Polytmus aquila, G. R. Gr. \& Mitch. Gen. of B. i. pl. 36. Glaucis aquila, $\operatorname{Pr} . B$. Eutoxeros aquila, Reichenb.

S. America (New Granada).

1084. Trochllus Condamini.

Trochilus Condamini, Boure.

S. America (Ecuador).

e. Campylopterus, $S w .1827$.

1085. Trochilus LaRgIPEN Nis.

B. M.

Trochilus largipennis, Bodd. $\quad$ Pl. enl. 672, f. 2. Vieill. Ois. dor. t. 5, 21. T. campylopterus et T. cinereus, Gm. T. latipennis, Lath. Less. Ois. Mouch. t. 34. Sw. Zool. Illustr. pl. 130, 131. Campylopterus latipennis, $S w$. Orthorhynchus latipennis, Cuv. Polytmus largipennis, G. R. Gr. Ornismya latipennis, et Trochilus (Campylopterus) latipennis, Less. Mellisuga latipennis, Steph. Lampornis latipennis, Hartl.

S. America (Guiana); West Indies (Tobago). 
1086. Trochilus ensipennis.

B. M.

'Trochilus ensipeunis, Sw. Zool. Illustr. pl. 107. Orthorhynchus ensipennis, Cuv. Campylopterus ensipennis, Sw. Trochilus (Campylopterus) ensipennis, Less. Polytmus ensipennis, G. R. Gr. Mellisuga ensipennis, Steph. Less. Ois. Mouch. t. 35. Troch.t. 46, 47.

S. America.

1087. Trochilus lazulus.

B. M.

Trochilus lazulus, Vieill. Gal. des Ois. t. 179. T. falcatus, Sw. Zool. Illustr. pl. 83. Less. Ois. Mouch. t. 36. Hylucharis lazulus, Boie. Ornismya falcata, Less. Orthorhynchus falcatus, Cuv. Mellisuga falcata, Steph. Polytmus lazulus, G. R. Gr. Trochilus (Campylopterus) falcatus, Less. Campylopterus falcatus, $S w$. C. lazulus, $\operatorname{Pr} . B$.

S. America (Venezuela).

1088. Trochilus hyperythrus.

Campylorhynchus hyperythrus, $\mathrm{Cab}$.

S. America (Guiaua).

1089. Trochildes cirrochloris.

B. M.

Trochilus cirruchloris, Vieill. T. campylostylus, Licht. Ornismya simplex, Less. Ois. Mouch. t. 33. Suppl. t. 6. Trochilus (Campylopterus) simplex, Less. Campylopterus cirrochloris, Jard. Polytmus cirrochloris, G. R. Gr.

S. America (Brazil).

1090. Trochilds rufus.

B. M.

Campylopterus rufus, Less. Polytmus rufus, G. R. Gr.

Central America (Guatimala).

1091. Trochilus DeLattre.

B. M.

Ornismya (Campylopterus) DeLattre, DeLattre \&. Less. Polytmus DeLattre, G. R. Gr. Campylopterus DeLattre, $\operatorname{Pr} . B$.

Central America (Mexico).

1092. Trochilus pampa.

B. M.

Ornismya pampa, Less. Ois. Mouch. Suppl. t. 15. Trochilus (Campylopterus) pampa, Less. Campylopterus pampa, Jard. Polytmus pampa, G.R. $G r$.

Central America (Mexico). 
1093. Trochilus Cuvieri.

Trochilus Cuvieri, DeLattre \& Bourc. Polytmus Cuvieri, G. R. Gr. Campylopterus Cuvieri, $\operatorname{Pr} . B$.

Central America (Panama).

1094. Trochilus obscurus.

Campylopterus obscurus, Gould. Polytmus obscurus, G. $R$. Gr.

S. America (River Amazon).

1095. Trochilus macrourus.

Trochilus macrourus, $G m$. T. forcipatus, Lath. Orthorhynchus forficatus, Cuv. Ornismya hirundinacea, Less. Ois. Mouch. t. 25. Suppl. t. 39. Trochilus (Campylopterus) hirundinaceus, Less. Cynanthus macrourus, $S w$. Melisuga macrourus, Boie. Polytmus macrourus, G. R. Gr.

S. America (Guiana, Brazil, Bolivia).

f. Glaucis, Boie, 1831.

1096. Trochilus hirsutus.

B. M.

Trochilus hirsutus, Gm. Vieill. Ois. dor. t. 20. Pl.col.120, f. 2. Less. Col.t. 21. 'T. brasiliensis, Lath. T. ferrugineus, Pr. Max. P T. mazeppa, Less. Troch. t. 3 (var.). Glaucis hirsutus, Boie. Trochilus (Glaucis) hirsutus, Less. Phætornis hirsutus, Jard. Nat. Libr. xv. pl. 29, Jard. \& Selby, Illustr. Orn. pl. 143. Polytmus hirsutus et P. mazeppa, G. $R$. $G r$.

S. America (Brazil).

$$
\begin{aligned}
& \text { g. Lampornis, Sw. } 1827 . \\
& \text { Polytmus, Vieill. } 1807 . \\
& \text { Anthracothorax, Boie, } 1831 .
\end{aligned}
$$

1097. Trochilus mango.

B. M.

Trochilus mango, $L . \quad P l . e n l .671$, f. $2 . \quad$ T. albus, $G m$. T. nitidus, Lath. 'T. violicauda, Bodd. T. fasciatus, Shaw. T. atricapillus, T. quadricolor, T. punctatus et T. nigricollis? Vieill. Lampornis mango, $S w$. Anthracothorax mango, Boie. Trochilus (Anthracothorax) mango, Less. Col.t. 12? 13, 14, 15. Polytmus mango, Vieill. Ois. dor. t. 7, 8, 11. Sw. B. of Braz. pl. 27, 28. Nat. Libr. xv. pl. 20.

S. America (Brazil? Bolivia); West Indies (Jamaica). 
1098. Trochilus PORPHYRURUS.

B. $M$.

Trochilus porphyrurus, Shaw, Gen. Zool. viii. pl. 40. Nat. Misc. pl. 333. 'T. Floresii, Bourc. T. bromicolor, Less. Polytmus porphyrurus, G.R. Gr. Lampornis mango, Gosse, $B$. of Jam. pl. 18. Lamporn is Floresii, Pr. B.

W. Indies (Jamaica).

1099. Trochilus dominicus.

B. $\mathrm{M}$.

Trochilus dominicus, $L_{*} \quad P l$. enl. 580, f. 2.621 , f. 1, 671, f. 1. T. gramineus et T. maculatus, $G m$. T. pectoralis et T. nitidus, Lath. T. gularis, Lath. T. viridigula, Bodd. T. marmoratus, Vieill. Ois. dor. t. 9, 10, 70. Anthracothorax gramineus, Boie. Trochilus (Anthracothorax) gramineus, Less. Col. t. 12, 12, bis. Lampornis gramineus, Jard. Nat. Libr. xv. pl. 32,33 . L. dominicus, $\operatorname{Pr} . B$. Polytmus dominicus, G. R. Gr. P. gramineus, Vieill.

West Indies (St. Domingo, Jamaica).

1100. Trochilus holosericeus.

B. M.

Trochilus holosericeus, $L . \quad E d w . B$. pl. $36 . \quad$ T. atrigaster, Shaw. Anthracothorax holosericeus, Boie. Trochilus (Anthracothorax) holosericeus, Less. Col. t. 20. Lampornis bolosericeus, Jard. Polytmus holosericeus, Vieill. Eulampis holosericeus, $P r . B$. Vieill. Ois. dor. t. 6, 65.

W. Indies (St. Thomas, Martinique, Porto Rico).

1101. ? Trochilus viridis.

Trochilus viridis, Vieill. Ois. dor. t. 15. T. aureo-viridis, Shaw. Trochilus (Anthracothorax) viridis, Less. Col. t. 11. Polytmus viridis, Vieill. Lampornis viridis, $\operatorname{Pr} . B$.

West Indies (Porto Rico).

1102. Trochilus margaritacets.

B. M. Trochilus margaritaceus et T. striatus? $\mathbf{G m}$. Pl. enl. $680, \mathrm{f}$. 1. T. hypophæus, Lath.? T. aurulentus, Vieill. Ois. dor. t. 12, 13, 16. Polytmus aurulentus, Vieill. P. margaritaceus, $G$. $R$. $G r$. Lampurnis margaritaceus, $\operatorname{Pr} . B$.

W. Indies (St. Domingo, Porto Rico). 
1103. Trochilus Prevostil.

B. M.

Trochilus Prevostii, Less. Col. t. 24. Trochilus (Anthracothorax) Prevostii, Less. Polytmus Preostii, G. R. Gr. Lampornis Prevostii, $\operatorname{Pr} . B$.

Surinam? West Indies (Tobago).

1104. Trochilus Leucurus.

B. $M$.

Trochilus leucurus, $L . \quad E d w . B$. pl. 256, f. 2. $P l$. enl. 600, f. 4. Less. Col. t. 22. T. ruficollis, Vieill. Trochilus (Anthracothorax) leucurus, Less. Polytmus leucurus, G. R. Gr. Glaucis leucurus, $\operatorname{Pr} . B$. Orthorhynchus ruficollis, $D^{\prime} O r b$. \& Lafi.

S. America (Guiana, Bolivia).

1105. Trochilus Antonia.

Trochilus Antoniæ, Bourc. \& Muls. Polytmus Antoniæ, G. $R$.

Gr. Lampornis Antoniæ, Pr. B.

S. America (Cayenne).

1106. Trochilus Ruckeri.

Trochilus Ruckeri, Bourc. Polytmus Ruckeri, G. R. Gr.

America (—?)

1107. Trochilus Buffonit.

B. M.

Trochilus Buffonii, Less. Troch. t. 5. Trochilus (Glaucis)

Buffonii, Less. Lampornis Buffonii, Jard. Nat. Libr. xv. pl. 19. Polytmus Buffunii, G. R. Gr.

S. America (Brazil).

1108. Trochilus cerdelegaster.

Trochilus (Glancis?) cæruleogaster, Gould. Polytmus cæruleogaster, G. R. Gr. Lamporuis cæruleogaster, Pr. B.

S. America (—?)

1109. Trochilus Cranopectus.

Trochilus (Lampornis) cyanopectus, Gould. Polytmus cyanopectus, G. R. Gr. Lampornis cyanopectus, Pr. B.

S. America (Venezuela). 
1110. Trochilus Schreibersit.

Trochilus Schreibersi, Lodd. Calothorax? Schreibersi, G. $R$. Gr. Thalurania Schreibersi, $\operatorname{Pr}, B$.

S. America (Brazil).

1111. Trochilus AURESCENS.

Trochilus (Lampornis) aurescens, Gould. Polytmus aurescens, G. $R$. Gr. Lampornis aurescens, $P r . B$.

S. America (Brazil).

1112. Trochilus cephalus.

Trochilus cephalus, Bourc. \& Muls.

Central America.

h. Eulampis, Boie, 1831.

1113. Trochilus Jugularis.

B. M.

Trochilus jugularis, $L . \quad E d w . B$. pl. 266, f. $1 . \quad$ T. auratus et T. cyanomelas, Gm. T. granatinus, T. Bancroftii et T. cyaneus, Lath. T. violaceus, Vieill. Ois. dor. t. 4. Eulampis jugularis et E. auratus, Boie. Trochilus (Eulampis) auratus, Less. Col. t. 10. Cynanthus? jugularis, Jard. Polytmus jugularis, G. R. Gr. Certhia prasinoptera, Sparr. Mus. Carls. t. 81. Lampornis granatinus, Hartl.

S. America (Guiana).

i. Petasophora, G. R. Gr. 1840 ; Pr. B. 1850.

Colibri, $\operatorname{Pr}$. B. 1850.

1114. Trochilds SERrirostris.

B. M.

Trochilus serrirostris, Vieill. Pl. col. 203, f. 3. T. petasophorus, Pr. Max. $\quad$ Colibri crispus, Spix, Av. Bras.t. 81, f. 1. Trochilus (Orthorhynchus) petasophorus, Cuv. Grypus? Vieillotii et Melisuga petasophora, Steph. Ornismya petasophora, Less. Ois. Mouch. t. 1. Troch. t. 12, 59. Trochilus (Ramphodon) petasophora, Less. Heliothrix petasophorus, Boie. Trochilus (Lophornis) petasophorus, Tschudi. Petasophora serrirostris et Polytmus serrirostris, G. R. Gr. Nat. Libr. xiv. pl. 13, xv. pl. 15. Lampornis petasophora, Hartl. Petasophora Gouldii, Pr. B. Consp. p. 69. Orthorhynchus petasophorus, D'Orb. \& Lafr.

S. America (Brazil, Bolivia). 
1115. Trochilus cyanotus.

B. M.

Trochilus cyanotus, Bourc. $\&$ Muls. Petasophora cyanotus, Gould. Polytmus cyanotus, G. R. Gr. Colibri cyanotis, Pr. B.

S. America (Venezuela).

1116. Trochilus thalassinus.

B. M.

Trochilus thalassinus, Sw. Less. Troch. t. 55, 56, 57. Ois. Mouch. t. 3. Petasophora thalassinus, Gould. Polytmus thalassinus, G. R. Gr. Cynanthus thalassinus, Jard. Nat. Libr. xv. pl. 1, 2. Colibri thalassinus, $\operatorname{Pr} . B$.

Central America (Mexico).

1117. ? Trochicus Jolatus.

B. M. Petasophora jolata, Gould. Polytmus iolatus, G. R. Gr. Colibri jolata, $\operatorname{Pr} . B$.

S. America (Bolivia).

1118. ? Trochilus coruscans.

Trochilus (Petasophora) coruscans, Gould. Petasophora coruscans, Gould. Polytmus cortuscans, G. R. Gr. Colibri corruscans, $\operatorname{Pr} . B$.

S. America ?)

1119. Trochilus anais.

B. M.

Ornismya anais, Less. Col. Supp. t. 3. Trochilus (Ramphodon) anais, Less. T. (Cæligeua) anais, Tschudi. Petasophora anais, Gould. Polytmus anais, G. R. Gr. Colibri anais, $\operatorname{Pr} . \mathcal{B}$.

S. America (Venezuela, New Granada).

1120. Trochilds DelphiNæ.

B. M.

Ornismya Delphinæ, Less. Petasophora delphinæ, Gould. Polytmus delphinus, G. R. Gr. Colibri delphinæ, Pr. B.

S. America. 
j. Polytmus, $B r .1760$.

Thaumatias, p. Pr. B. 1850.

1121. Trochilus thadmantias.

B. M.

Trochilus thaumantias, $L . \quad P l$. enl. 600, f. $1 . \quad$ T. leucogaster, Gm. Pl. enl.600, f. 2. T. tephrocephalus, Vieill. Trochilus (Basilinna) albiventris et T. (B.) tephrocephalon, Less. Oruismya albiventris, Less. Ois. Mouch. t. 76. Troch. t. 32. O. tephrocephalon, Less. Ois. Mouch. t. 70. Glaucis thaunantias, Boie. Polytmus thaumatias et P. tephrocephalus, $G$. $R$. $G r$. Thaumatias albiventris, $\operatorname{Pr} . B$.

S. America (Brazil, Guiana).

1122. Trochilus albirostris.

B. M.

Ornismya albirostris, Less. Ois. Mouch. t. 78. Trochilus (Basiliuna) albirostris, Less. Trochilus leucogaster, Vieill. Ois. dor. t. 43.

S. America (Guiana).

1123. Trochiles albicollis.

B. M.

Trochilus albicollis, Vieill. $P l$. col. 203, f. 2. Orthorhynchus albicollis, Cuv. Trochilus (Basilinna) albicollis, Less. Ornismya albicollis, Less. Ois. Mouch. t. 63. Colibri albogularis, Spix, Av. Bras. t. 82, f. 1. Basilinna albicollis, Boie. Polytmus albicollis, G. R. Gr. Thaumatias alhicollis, $\operatorname{Pr} . B$.

S. America (Brazil).

1124. Trochilus BREVIROSTRIS.

B. M.

Ornismya brevirostris, Less. Ois. Mouch. t. 77. O. senex, Less. Trochilus (Basilinna) brevirostris, Less. Polytmus brevirostris et P. senex, G. R. Gr. Thaumatias brevirostris, Pr. B.

S. America (Guiana).

1125. Trochilds Millerii.

Trochilus Millerii, Lodd. Polytmus Milleri, G. R. Gr. Thaumatias Milleri, $\operatorname{Pr} . B$.

S. America (Brazil). 
126. Trochilus candidus. B. M.

Trochilus candidus, Bourc. \& Muls. Polytmus candidus, $G$. R. Gr. Thaumatias candidus, $P r . B$.

Central America (Guatimala).

1127. Trochilus aureoventris.

Orthorhynchus aureorentris, D'Orb. \& Lafr. Polytwus aureoventris, $G, R$. $G r$.

S. America

1128. TROCHILUS VIRIDISSIMUS.

B. M.

Trochilus viridissimus, $\mathrm{Gm}$. T. viridicaudus, Saucer. Ornismya viridis, Less. Troch. t. 33. Ornismya (Basilinna) viridis, Less. Smaragdites viridissimus, Boie. Polytmus viridissimus, G. R. Gr. Amazilius viridissimus, Pr. B.

Trinidad.

1129. Trochilus viridis.

B. M.

Trochilus viridis, Vieill. Ois. dor. t. 41. T. chrysobronchus, Shaw. T. chlorolencurus, Saucer. T. virescens, Dum. Orthorhynchus viridis, Cuv. Ornismya viridis, Less. Ois. Mouch. t. 60. Ornismya (Basilinna) viridis, Less. Polytmus chrysobronchus, G. R. Gr. Phœthornis chrysobronchos, Sw.

S. America (Guiana).

1130. Trochilus chlorurus.

B. M.

Trochilus viridissimus, Vieill. Ois. dor. t. 42. Ornismya viridissima, Less. Ois. Mouch. t. 75. O. albicollis, p. 'D'Orb. \& Lafr. Trochilus (Basilinna) viridissimus, Less. Polytmus, sp. No. 5 l, G. R. Gr.

S. America (Brazil).

1131. Trochilos Hillit.

Trochilus Mariæ, Hill. Gosse, B. of Jam. pl. 22.

W. Indies (Jamaica).

1132. ? Trochilus CINEREICOLIS.

Trochilus cinereicollis, Vieill. Polytmus cinereicollis, $G . R$. Gr.

S. America (Paraguay). 
1133. ? Trochilus cyanocaudus.

Trochilus cyanurus, Vieill. Polytmus cyanurus, G. R. Gr.

S. America (Paraguay).

\section{k. Leucippus, $\operatorname{Pr}$. B. 1850.}

1134. Trochilus fallax.

B. M.

Trochilus fallax, Bourc. Trochilus (Lampornis) fulviventris, Gould. Polytmus fallax, G. R. Gr. Leucippus fallax, Pr. B.

S. America (Venezuela).

1135. Trochilus chionogaster.

Trochilus leucogaster et T. chionogaster, Tschudi, Fauna Per. t. 22, f. 2. T. (-?) hypoleucus, Gould. T. Turneri, Bourc. Polytmus chionogaster et P. hypoleucus, G. R. Gr. Thaumatias leucogaster et Leucippus Turneri, $\operatorname{Pr}$. B.

S. America (Peru).

\section{Trochilus viridi-Pallens.}

Trochilus viridi-pallens, Bourc. \& Muls. Polytmus viridi-pallens, G. R. Gr. Delattria viridi-pallens, $\operatorname{Pr} . B$.

Central America (Guatimala).

1137. Trochilds glaucopis. B. M. Trochilus glaucopis, $G m . \quad$ Briss. Orn. iii. t. 36, f. 5. T. frontalis, Lath. T. pileatus, $\operatorname{Pr}$. Max. Trochilus (Mellisuga) glaucopis, Less. Ornismya glaucopis, Less. Ois. Mouch. t. 58, 59. Mellisuga glaucopis, Boie. Cynanthus glaucopis, Jard. Polytmus glaucopis, G. R. Gr. Thalurania glaucopis, Pr. B.

S. America (Brazil).

1138. Trochilus gladcopoides.

Orthorhynchus glaucopoïdes, D'Orb. \& Lafr. Trochilus D'Orbignyi, Bourc. Polytmus glaucopoïdes et Hylocharis D'Orbignyi, G.R. Gr.

S. America (Bolivia). 
1139. Trochilus columirus.

B. M.

Ornismya columbica, Bourc. Ann. Sci. Phys. \&c. 1843, t. 4. Polytmus columbicus, G. R. Gr.

S. America.

\section{$\longrightarrow$}

1140. TrochIlus QUadricolor. B. M. Trochilus quadricolor, Vieill. Ornismya cyanocephala, Less. Ois. Mouch. Suppl. t. 18. Polytmus quadricolor, G. R. Gr.

S. America (Brazil).

1141. Trochilus verticalis.

B. M.

Ornismya cyanocephala, Less. Ois. Mouch. Suppl. t. 17. Trochilus verticalis, Licht. Polytmus verticalis, G.R. Gr. Nat. Libr. xiv. pl. 9.

S. America (Brazil).

1142. 'Trochilus Francia.

B. M.

Trochilus Franciæ, Bourc. \& Muls. Polytmus Franciæ, G. R. Gr.

S. America (New Granada).

1. Glaucopes, Less. 1829.

Thalurania, Gould, 1848.

1143. Trochilus furcates.

B. M.

Trochilus furcatus, Gm. Pl. enl. 599, f. 2. Vieill. Ois, dor. t. 34. T. elegans, Shaw, Nat. Misc. pl. 397. Trochilus (Mellisuga) furcatus, Less. T. (Cæligena) furcatus, Tschudi. Orthorhynchus furcatus, Cuv. Ornismya furcata, Less. Ois. Mouch. t. 18. Cynanthus furcatus, Jard. Nat. Libr. xir. pl. 23. Polytmus furcatus, G. R. Gr. Thalurania furcata, Gould.

S. America (Guiana, Brazil).

1144. Trochilus Nigrofasciatus.

Trochilus (- nigrofasciatus, Gould. Polytmus nigrofasciatus, G. R. Gr. Thalurania nigrofasciata, Gould.

S. America (Brazil). 
1145. Trochilus viridipectus.

Thalurania viridipectus, Gould. Polytmus viridipectus, G. R. $G r$.

S. America (Columbian Andes).

1146. Trochilus eriphile.

B. M.

Ornismya eriphile, Less. Ois. Mouch. Suppl. t. 25. 'Trochilus (Mellisuga) eriphile, Less. Polytmus eriphile, G. R. Gr. Thalurania eriphile, $\operatorname{Pr} . B$.

S. America (Brazil).

1147. Trochilus Waterion,

Trochilus Watertonii, Bourc. Polytmus Watertoni, G. R. Gr. Thalurania Watertoni, Gould.

S. America (Guiana).

1148. Trochilus cyanifrons. Trochilus cyanifrons, Bourc. Polytmus cyanifrons, G. R. Gr. Thalurania cyanifrons, $\mathrm{Pr}$. B.

S. America (New Granada).

1149. Trochilus Wagleri.

B. M.

Ornismya Wagleri, Less. Ois. Mouch. t. 73. Trochilus (Mellisuga) Wagleri, Less. Trochilus sapphirinus, Vieill. Ois, dor. t. 57. Cynanthus Wagleri, Jard. Nat. Libr. xv. pl. 16. Hylocharis Wagleri, G. R. Gr. Thalurania Wagleri, Pr. B.

S. America (Brazil).

1150. Trochilus Fanny.

Trochilus Fanny, Bourc. \& Muls. Hylocharis Fanny, G. R. Gr.

S. America.

1151. ?'Trochilus FURCIFER.

Trochilus furcifer, Shaw. T. caudacutus, Vieill. Polytmus furcifer, G.R. Gr.

S. America (Paraguay). 
m. Topaza, G. R. Gr. 1840.

Topazes, Less. 1829.

1152. Trochilus peita.

B. M.

Trochilus pella et T. paradiseus, $L . \quad P l$. enl. 599, f. $1,2$. $E d w . B$. pl. 33. Ois. dor. t. 2. T. violaceus, Gm. Lampornis pella, Sw. Polytmus pella et Eulampis violaceus, Boie. Troehilus (Les Topazes) pella, Less. Less. Col. t. 2, 3, 4, 5. Nat. Libr. xv. pl. 24, 25. Topaza pella, G. R. Gr.

S. America (Guiana).

1153. Trochilus pyra.

Trochilus pyra, Gould. Topaza pyra, G. R. Gr.

S. America (Brazil).

n. Florisuga, $\operatorname{Pr}$. B. 1850.

1154. Trochilus fuscus.

B. M.

Trochilus ater, Pr. Max. T. fuscus, Vieill. T. niger, Sw. Zool. Illustr. pl. 82. T. atratus, Licht. Lampornis niger, Sw. L. atratus, Hartl. Mellisuga atra, Steph. Ornismya lugubris, Less. Ois. Mouch. t. 38, 39 . Colibri leucopygius, Spix, Av. Bras. t. 81, f. 3. Trochilus (Eulampis) lugubris, Less. Eulampis niger, Boie. Topaza atra, G. R. Gr. Florisuga atra, $\operatorname{Pr} . B$.

S. America (Brazil).

1155. Trochilus opacus.

Trochilus opacus, Licht. Topaza opaca, G. R. Gr. Trochilus (Lampornis) opacus, Tschudi.

S. America (Peru).

1156. Trochilus mellivorus.

B. M.

Trochilus mellivorus, $L . \quad P l$. enl. 640, f. 2 . T. fimbriatus, Gm.? Pl. enl. 276, f. 2? Edw. B. pl. 35, f. 1. Mellisuga surinamensis, Steph. Basilinna mellivorus, Boie. Vieill. Ois. dor. t. 22, 23, 24. Ornismya mellivora, Less. Ois. Mouch. dor. t. 22, 23, 24. Ornismya mestives Jacobines) mellivorus, Less. Or-
t. 21, 22. Trochilus (Les,
thorhynchus mellivorus, Cuv. Lampornis mellivorus, Jard. Nat. Libr. xiv. pl. 30. Topaza mellivora, G.R. Gr. Florisuga mellivora, $\operatorname{Pr} . \boldsymbol{B}$.

S. America (Guiana); W. Indies (Martinique). 
1157. Trochilus Flabelliferus.

Trochilus ( $\longrightarrow$ ?) flabelliferus, Gould. Topaza Aabellifera, G. R. Gr. Florisuga flabellifera, Pr. B.

Central America (Mexico).

1158. Trochilus Henrica.

B. M.

Ornismya Henrica, Less. \& DeLattr. Topaza henrica, G. R.

$G r$. Delattria henrica, Pr. B. Trochilus henrica, Hartl.

Central America (Mexico).

1159. Trochilus Caroli.

Trochilus Caroli, Bourc. Hylocharis Caroli, G. R. Grr.

S. America.

o. Lafresnaya, Pr. B. 1850.

1160. Trochilus Lafresnayi.

B. MI.

Trochilus Lafresnayi, Bross. T. flavicaudatus, Fras. Calothorax Lafresnayi, G. R. Gr. Lafresnaya flavicaudatus, Pr. B. Phætornis Lafrenagi, Hartl.

S. America (New Granada).

1161. Trochilus Gayr.

Truchilus Gayi, Bourc. \& Muls. T. Saulii, DeLattr. \& Bourc. Calothorax Gayi et C. Saulii, G.R. Gr. Lafresnaya Gayi et L. Saulæ, $\operatorname{Pr}, B$.

S. America (Ecuador)

$$
\text { p. Thaumastura, Pr. B. } 1850 .
$$

1162. Trochilus Cora.

B. M.

Orthorhynchus cora, Less. \& Garn. Voy. de la Coqu. t. 31, f. 4. Trochilus (Heliactin) cora, Less. T. (Phætornis) cora, Tschudi. Ornismya cora, Less. Ois. Mouch. t. 6. Troch, t. 39, 40. Heliactin cora, Boie. Calothorax cora, G. R. Gr r. Thaumastura cora, Pr. B. Nat. Libr. xiv. pl. 25.

S. America (Peru). 
1163. Trochilus Labrador.

Ornismya fanny, Less. Trochilus labrador, Bourc. An. Sc. Phys. \& c. de ĹLyon, 1838, t. Calothorax fanny, G. R. Gr. Thaumastura fanny, $\operatorname{Pr} . B$.

Central America (Mexico).

1164. Trochilus Yarreliti.

Trochilus Yarrellii, Bourc. Calothorax Yarrellii, G. R. Gr.

S. America (Banda Oriental).

1165. Trochilus vesper.

B. M.

Ornismya vesper, Less. Ois. Mouch, t. 19. Troch. t. 6, 48. Trochilus (Les Lucifers) vesper, Less. Cynanthus vesper, Jard. Nat. Libr. xiv. pl. 24. Calothorax vesper, G. R. Gr. Thaumastura vesper, $\mathrm{Pr}$. $B$.

Central America (Mexico); S. America (Peru).

1166. TrochilUS ANgele.

B. M.

Ornismya angelæ, Less. Illustr. de Zool. t. 49, 50. Trochilus Leadbeateri, Lodd. MSS. T. Buquetii, Par. Mus. Calothorax angelæ, G.R. Gr. Heliomaster angelæ, Pr. B.

S. America (Chili).

q. Calothorax, G. R. Gr. 1840.

Lucifers, Less. 1829.

1167. Trochilds enicurus.

Trochilus enicurus, Vieill. Pl col 66, 3 B. M. ropygia, Less. Ois. Mouch. t. 15. Trochilus (Heliactin) heteropygia, Less. Orthorhynchus enicurus, Cuv. Cynanthus enicurus, Jard. Nat. Libr. xiv. t. 27. Calothorax enicurus, G. R. Gr. Steph. Gen. Zool. xiv. pl. 28.

S. America (Brazil); West Indies (Trinidad?). 
1168. Trochilus LUCIFER.

B. M.

Cynanthus lucifer, Sw. Nat. Libr. xv. pl. 14. Ornismya cyanopogon, Less. Ois. Mouch. t. 5. Col. Suppl. t. 9, 10. Trochilus simplex, Less. Col. t. 23. Trochilus (Les Lucifers) cyanopogon et Tr. (Glancis) simplex, Less. Caluthorax lucifer et Polytmus simplex, G. R. Gr.

Central America (Mexico); S. America (Bolivia).

1169. Trochilus Eliza.

B. M.

Trochilus Eliza, DeLattr. \& Less. Calothorax Eliza, G. $R$. $G r$.

Central America (Mexico).

1170. Trochitus Evelyne.

Trochilus Evelynæ, Bourc. Calothorax Evelynæ, G. R. Gr.

Bahama Islands (New Providence).

r. Docimaster, Gould, 1850.

1171. Trochilus ensiferus.

B. M.

Ornismya ensifera, Boiss. Mag. de Zool. 1840. Ois. t. 15 . Trochilus Derbianus, Fras. T. ensiferus, Hartl. Mellisuga ensifera, G. R. Gr. Docimaster ensiferus, Gould.

S. America (New Granada, Ecuador).

s. Delattria, $\operatorname{Pr}$. B. 1850.

1172. Trochilus FULGENS.

B. M.

Trochilus fulgens, Sw. Nat. Libr. xv. pl. 18. Ornismya

Rivolii, Less. Ois Mouch. t. 4, Trochilus (Cæligena) Rivolii, Less. Mellisuga fulgens, G.R. Gr. Delattria fulgens, Pr. B.

Central America (Mexico).

1173. Trochilus Clemencie.

Ornismya Clemenciæ, Less. Ois. Mouch. t. 80. Col. Suppl. t. 8. Trochilus (Cæligena) Clemenciæ, Less. Mellisuga Clemencix, G. R. Gr. Delattria clemenciæ, $P r . B$.

Central America (Mexico). 
t. Heliodoxa, Gould, 1849.

Leadbeatera, $P r . B .1850$.

1174. Trochilus Leadbeateri.

B. M.

Trochilus Leadheateri, Bourc. Ann. Sci. Phys. \&c. de Lyon, 1843, t. 5. T. Otero et T. (Lampornis) Otero, T'schudi. Mellisuga Leadbeateri, G. R. Gr Heliodoxa Leadbeateri, Gould. Leadbeatera grata, $\operatorname{Pr}$. $B$.

S. America (Venezuela).

1175. Trochilus Jacurus.

Heliodoxa jacula, Gould. Leadbeatera jacula, Pr. B.

S. America (New Granadia).

1176. Trochilus Jamesoni.

Trochilus Jamesoni, Bourc.

S. America (Ecuador).

1177. Trochilus Bougueri.

Trochilus Bongueri, Bourc.

S. America (Ecuadoŕ).

1178. Trochiles SchiodteI.

Trochilus Schiodtei, Bourc. \& Muls.

S. America (Ecuador).

u. Heliomaster, Pr. B. 1850.

1179. Trochiles LONGIRostris.

B. M.

Trochilus longirostris, Vieill. Ois. dor. t. 59. T. superbus, Shaw, Nat. Misc. pl. 517. Orthorhynchus superbus, Cav. Trochilus (Cæligena) superba, Less. Ornismya superba, Less. Ois. Mouch. t. 2. Troch. t. 34. Col. Suppl. t. 33. Mellisuga superba, Steph. M. longirostris, G.R. Gr. Calliphlox longirostris, Boie. Heliomaster longirostris, Pr. B. Temm. $\quad \stackrel{P l}{\text { : }}$ col. 299 , f. 1 .

Island of Trinidad; S. America (Bolivia). 
1180. Trochilus mesoledeus.

B. M.

Trochilus mesoleucus, Temm. $\mathrm{Pl}$. col. 317, f. 1, 2, 3. T. squamosus, Temm. Pl. col. 203, f. 1. Orthorhynchus mesoleucus, Cuv. Trochilus (Cæligena) mesoleucos, Less. Ornismya Temminckii et O. mesoleuca, Less. Ois. Mouch. t. 20, 29, 30. Troch. t. 45. Mellisuga mesolenca et M. squamosa, Steph. Calliphlox mesoleucus, Boie. Heliomaster mesoleucus, Pr. B. Nat. Libr. xv. p. 17.

S. America (Brazil).

1181. Trochilus Villavicensis.

Trochilus Villavicensis, Bourc.

S. America (Ecuador).

v. Helianthea, Gould, 1848.

1182. Trochilus heliantheus.

B. M.

Ornismya helianthea, Less. Mellisuga helianthea, G. R. Gr. Helianthea typica, Pr. B. Trochilus heliantheus, Hartl.

S. America (New Granada).

1183. Trochilus Lutetia.

Trochilus Lutetiæ, DeLattr. \& Bourc. Mellisuga Lutetiæ, G.

R. $G r$. Helianthea Lutetiæ, $\operatorname{Pr} . B$.

S. America (New Granada).

1184. Trochilus Bonaparter.

B. M.

Ornismya Bonapartei, Boiss. Trochilus aurogaster, Lodd. Mellisuga Bonapartei, G. R. Gr. Helianthea Bonapartei, Gould. Ann. Sci. Phys. \&ce de Lyon, 1842, t. 14.

S. America (New Granada).

1185. Trochilus Eos.

Helianthea Eos, Gould, Proc. Z. S. 1848, p]. 1. Mellisuga Eos, G. R. Gr.

Mountains of New Granada and Venezuela.

1186. Trochilus violifer.

Trochilus (- ?) violifer, Gould. Mellisuga violifera, $G$. R. Gr. Helianthea violifera, $\operatorname{Pr} . B$.

S. America (Bolivia). 
x. Pterophanes, Gould, 1849.

1187. Trochilus Temminchit.

B. M.

Ornismya Temminckii, Boiss. Mag. de Zool. 1840. Ois. t. 14. Trochilus cyanopterus, Lodd. Mellisuga Temminckii, G. R. Gr. Pterophanes Temminckii, $\operatorname{Pr} . B$.

S. America (New Granada).

y. Bourcieria, $\operatorname{Pr}, B .1850$.

1188. Trochilus Prunelli.

B. M.

Trochilus Prunellii, Bourc. \& Muls. Ann. Sci. Phys. 1843, t. 1. Mellisuga Prunellii, G. R. Gr. Bourcieria Prunellii, $\operatorname{Pr} . B$.

S. America (Columbia).

1189. Trochilus torquatus.

B. M.

Ornismya torquata, Boiss. Trochilus insectivorus, Tschudi, Fauna Per. t. 23, f. 1. Mellisuga torquata, G. R. Gr. Bourcieria torquata, $\operatorname{Pr} . B$.

S. America (Peru).

1190. Trochilus Wilsoni.

Trochilus Wilsoni, DeLattr. \& Bourc. Mellisuga Wilsoni, G. R. Gr. Bourcieria Wilsoni, Pr. B.

S. America (Néw Granarla).

1191. Trochilus Conradi.

Trochilus Conradi, Bourc. Mellisuga Conradii, G. R. Gr. Bourcieria Conradi, $\operatorname{Pr} . B$.

S. America (Venezuela).

z. Cæligena, Less. 1829.

1192. TrochilUS CELIGENA.

B. M.

Ornismya cæligena, Less. Troch. t. 53. Trochilus (Cæligena) Less. Lampornis cæligena, Jard. Nat. Libr. pl. 4. Mellisuga cæligena, G.R. Gr. Cæligena typica, Pr. B.

Central America (Mexico). 
a, a. Heliangelus, Gould.

1193. Trochilus CLARISSE. B. $M$.

Ornismya clarissæ, Longuem. Mag. de Zool. 1842. Ois. t. 46. Mellisuga clarissæ, G. R. Gr. Heliangelus clarisse, Gould. Trochilus clarissæ, Hartl.

S. America (New Granada).

1194. Trochilus Spencei.

Trochilus Spencei, Bourc. Mellisuga Spencei, G. R. Gr. Heliangelus Spencei, Gould.

S. America (Venezuela).

1195. Trochilus mavors.

Heliangelus mavors, Gould, Proc. Z. S. 1848, pl. 2. Mellisuga mavors, G. R. Gr.

Cordilleras of New Granada and Venezuela.

1196. Trochilus Parzudaki.

B. M.

Ornismya Parzudaki, Less. Trochilus exortis, Frus. Mellisuga Parzudaki et M. exortis, G. R. Gr. Heliangelus Parzudaki et Cynanthus vel Eriopus exortis, $\operatorname{Pr}$. $B$.

S. America (New Granada).

1197. Trochilus strophianus.

Trochilus ( - ?) strophianus, Gould. Mellisuga strophiana, G. R. Gr. Heliangelus strophianus, Gould.

S. America (Ecuador).

1198. Trochilus AMETHYSTICOLlis.

B. M.

Orthorhynchus amethysticollis, D'Orb. \& Lafr. Voy. dans l'Amer. Mer. Ois. t. 60, f. 2. Trochilus (Lampornis) amethysticollis, Tschudi. Mellisuga amethysticollis, G.R. Gr. Heliangelus amethysticollis, $\operatorname{Pr}$. B.

S. America (Bolivia). 


\section{b, b. Doryfera, Gould, 1847.}

1199. Trochilus Lunovicie.

Trochilus Ludoviciæ, Bourc. \& Muls. Mellisuga Ludoviciæ, G. R. Gr. Trochilus (Doryfera) Louise, Gould? Dorifera Ludoviciæ, $\operatorname{Pr} . B$.

S. America.

1200. Trochilus JohanNz.

Trochilus Johannæ, Bourc. Trochilus (Doryfera) Johannæ,

Gould. Mellisuga johannæ, G. R. Gr.

S. America (Peru).

1201. Trochilus violifrons.

Trochilus (Doryfera) violifrons, Gould. Mellisuga violifrons, G. R. Gr.

S. America.

1202. Trochilus Rubineus.

B. M.

Trochilus rubineus, Lath. Pl. enl. 274, f. 4. Ois. dor. t. 28. T. obscurus, Gm. T. ruficaudatus, Vieill. T. rubineus major, Vieill. Ois. dor. t. 27. Orthorhynchus rubineus, Cuv. . Trochilus (Calliphlox) rubineus, Less. Mellisuga rubinea, Steph. Calliphlox ruficaudatus, Boie. Ornismya rubinea, Less. Ois. Mouch. t. 44, 45, 46. Cynanthus rubineus, $J$ ard. Heliomaster rubineus, $\operatorname{Pr}$. $B$. Heliodoxa? rubinea, Gould.

S. America (Guiana).

\section{TrochILUS RUBINOIDES.}

Trochilus rubinoïdes, Bourc. \& Muls. Heliodoxa rubinoïdes, Gould. Heliomaster rubinoïdes, $\operatorname{Pr}, B$.

S. America (New Granada).

1204. Trochilus rhami. B. M.

Ornismya rhami, Less. Mellisuga rhami, G.R. Gr. Lampornis? rhami, Pr. B. Trochilus rhami, Hartl.

Central America (Mexico). 
1205. Trochilus flavescens.

B. M.

Trochilus flavesceus, Lodd. T. fuscicaudatus, Fras. Ornismya paradisea, Boiss. Mellisuga flavescens et Hylocharis fuscicaudata, $G . R$. Gr. Amazilius flavescens, $\operatorname{Pr} . B$.

S. America (New Granada).

1206. Trochilus Jardiner.

Trochilus Jardinei, Bourc.

S. America (Ecuador).

1207. Trochilds Matrhewsit.

Trochilus Matthewsii, Lodd. et Bourc. Mellisuga Matthewsii, G. R. Gr.

S. America (Peru).

c, c. Aglæactis, Gould, 1848.

1208. Trochilus CUPRIPENNis.

B. $\mathrm{M}$.

Trochilus cupripennis, Bourc. et Muls. Mellisuga cupripennis et M. caumatonota, G. R. Gr. Aglæactis cupripennis et A. caumatonotus, Gould.

S. America (New Granada).

1209. Trochilus Castelnaudi.

Trochilus Castelnaudii, Bourc. \& Muls. Aglæactis Castelnaudi, $\operatorname{Pr} . B$.

S. America (- ?).

1210. Trochilus pamela.

B. .11.

Orthorhynchus pamela, D'Orb. \& Lafr. Vry. dans l'Amer. $M e r$. Ois. t. 6 o, f. 1. Hylocharis pamela, G. $\mathscr{R}$. Gr. Aglæactis pamela, Gould.

S. America.

$$
\text { d, d. Metallura, Gould, 184\%. }
$$

1211. Trochilus smaragdinicollis.

B. M.

Orthorhynchus smaragdinicollis, D'Orb. \& Lafr. Voy. dans l'Amer. Mer. Ois. t. 59, f. 1 . Mellisuga smaragdinicollis, $G$. R. Gr. Metallura smaragdinicollis, $\operatorname{Pr}, \boldsymbol{B}$.

S. America (Bolivia). 
1212. Trochilus cUPricaUda.

B. M.

Trochilus (- ?) cupricauda, Gould. Mellisuga cupreocauda, G. R. Gr. Metallura cupreocauda, Gould.

S. America (Bolivia).

1213. Trochilus anEOCAUDA.

B. M.

Trochilus ( - ?) æneocauda, Gould. Mellisuga æneocauda, G. R. Gr. Metallıra æneocauda, Gould.

S. America (Bolivia).

\section{Trochilus Williami.}

Trochilus Williami, Bourc. Mellisuga Williami, G. R. Gr. Metallura Williami, Gould.

S. America (New Granada).

1215. Trochilus tyrianthinus.

B. $\mathrm{M}$.

Ornismya Allardi, Bourc. Ann. Sci. Phys. \&c. de Lyon, 1840 , t. 3. O. Paulinæ, Boiss. Mag. de Zool. 1840. Ois. t. 13. Trochilus tyrianthinus, Lodd. Mellisuga tyrianthinus, $G$. $R$. $G r$. Metallura Allardi, Gould. M. tyrianthinus, $\operatorname{Pr} . B$.

S. America (New Granada).

\section{Trochilus Benjamint.}

Trochilus Benjamini, Bourc.

S. America (Ecuador).

$$
\text { e, e. Oxypogon, Gould, } 1848 .
$$

1217. Trochilus Guerinit.

B. M.

Ornismya Guerinii, Boiss. Trochilus parvirostris, Fras. T. Guerini, Bourc. Mellisuga Guerinii, G.R. Gr. Oxypogon Guerinii, Gould, Monogr. Troch. i. pl. 11.

S. America (New Granada).

1218. Trochilus Lindeni. B. M. Ornismya Lindenii, Parzud. Rev. et Mag. de Zool. 1849, t. 8. Mellisuga Lindenii, G. R. Gr. Oxypogon Lindenii, Gould, Monogr. Troch. i. pl. 12.

S. America (Venezuela). 
1219. Trochilus heteropogon.

B. M.

Ornismya heteropogon, Boiss. May. de Zool. 1840. Ois. t. 12. Trochilus corruscans, Fras. Mellisuga heteropogon, G. $R$. $G r$. Ramphomicron heteropogon, $\boldsymbol{P r} . \boldsymbol{B}$.

S. America (New Granada).

1220. Trochilus Rufice PS.

Trochilus (- ?) ruficeps, Gould. Ramphomicron ruficeps, Pr. B.

S. America (Bolivia).

1221. Trochilus Herrani.

Trochilus Herrani, Delattr. \& Bourc. Calothorax Herrani, G. R. Gr.

S. America (New Granada).

1222. Trochilus Stanleyi.

B. M.

Trochilus Stanleyi, Bourc.

S. America (Ecuador).

f, f. Ramphomicron, Pr. B. 1850.

1223. Trochilus Microrhynchus.

B. $M$.

Ornismya microrhyncha, Boiss. Mag. de Zool. 1840. Ois. t. 16. Trochilus brachyrhynchus, Fras. Mellisuga microrhyncha, $G$. R. Gr. Ramphomicron microrhynchus, $P r . B$. Trochilus microrhynchus, Hartl.

S. America (New Granada).

1224. Trochilus Sabine.

B. $\mathbf{M}$.

Trochilus Sabinæ, Bourc. Mellisuga Sabinæ, G. R. Gr. Ramphomicron Sabinæ, Pr. B.

S. America (New Granada).

1225. Trochilus inornatus.

B. M.

Trochilus (- ${ }^{\text {? }) ~ i n o r n a t a, ~ G o u l d . ~ M e l l i s u g a ~ i n o r n a t a, ~}$ G. R. Gr. Ramphomicron inornatus, $P r . B$.

S. America (Bolivia).

1226. Trochilus melanogenys.

Trochilus melanogenys, Fras. Ramphomicron melanogenys, Pr. B.

S. America (New Granada). 
g, g. Abeillia, $\operatorname{Pr} . B .1850$.

1227. Trochilus Abeillit.

B. M.

Ornismya Abeillii, De'attr. et Less. Mellisuga Abeillii, G. $R$.

Gr. Ramphomicron Abeillii et Abeilla typica, Pr. B.

Central America (Mexico).

h, h. Cometes, Gould, 1847.

Sappho, Reichenb. 1849.

1228. Trochllus sparganurus.

B. M.

Trochilus sparganurus, Shaw, Gen. Zool. viii. pl. 39. T. chrysurus, Cuv. T. chrysochloris, Vieill. T. radiosus, Temm. Ornismya Sapho, Less. Ois. Mouch. t. 27, 28. Troch. t. 49. Trochilus (Lesbia) Sapho, Less. T. (Cynanthus) chrysurus, Tschudi. Orthorhynchus chrysurus, D'Orb. \& Lafr. Cynanthus sparganurus, Jard. Nat. Libr. Xv. pl. 23. Cometes sparganurus, Pr. B. C. Sapho, Gould. Mellisuga sparganura, G. R. Gr.

S. America (Peru? Bolivia).

1229. Trochilus phaon.

Cometes phaon, Gould. Mellisuga phaon, G. R. Gr.

S. America (Peru).

i, i. Lesbia, Less. 1829 .

Cynanthus, Pr. B. 1850.

B. M.

1230. Trochilus Bifurcatus.

Cynanthus bifurcatus, $S w$. C. nuna, Jard. Ornismra nuna, Less. Ois. Mouch. Suppl. t. 35. Trochilus (Leshia) nuna, Less. Mellisuga bifurcata, G, R. Gr.

Central America (Mexico)? S. America (Chili? Peru, New Granada).

1231. Trochilus Amaryluis.

Trochilus Amaryllis, Bourc. \& Muls.

S. America (New Granada). 
1232. Trochilus Forficatus.

B. II.

Trochilus forficatus, $L . E d w . B . p l$. 33. Ois. dor. t. 60 . T. cyanurus, L.? Steph. T. bipartitus, Lath. MSS. Ornismya Kingii, Less. Troch. t. 38. Trochilus (Lesbia) Kingii, Less. Mellisuga forficata et M. cyanura, G. R. Gr. Orthorhynchus forficatus, Cuv. Cynanthus forficatus, $\operatorname{Pr}_{\mathbf{r}} B$.

S. America (Guiana? Chili): West Indies (Jamaica)?

1233. Trochiles Mocos.

B. M.

Trochilus mocoa, Bourc. Truchilus (Lesbia) smaragdinus, Gould. Mellisuga smaragdinus, G. R. Gr. Cynanthus mocoa, $\operatorname{Pr}, B$.

S. America (New Granada, Bolivia).

1234. Trochilus Silphia.

B. M.

Ornismya silphia, Less. Trochilus Gouldii, Lodd. T. silphia, Hartl. Mellisuga Gouldii, G. R. Gr. Cynanthus Gouldi, Pr. B. Trochilus (Lesbia) gracilis, Gould. Mellisuga gracilis, G. R. Gr. Cynanthus gracilis, $\operatorname{Pr} . B$.

S. America (New Granada, Peru).

1235. Trochilus Victoria.

Trochilus Victoriæ, Bourc. Rev. Zool. 1846, t. 4. Mellisuga Victorix, G. R. Gr. Cynanthus Victoriæ, Pr. B.

S. America (New Granada).

1236. Trochilus Eucharis.

Trochilus Eucharis, Bourc. \&. Muls.

S. America ?)

j, j. Platures, Less. 1829.

Discosura, Pr. B. 1850.

Ocreatus, p. Gould, 1846.

1237. Trochiles longicaedes.

B. M.

Trochilus longicaudus, Gm. T. platurus, Lath. Ois. dor. $\mathrm{t}$. 52. Smaragdites longicaudus, Boie. Orthorhynchus platurus, Cuv. Trochilus (Platurus) - , Less. Ornismya platura, Less. Ois. Mouch. t. 40. Col. Suppl. 1. 31. Cynanthus platurus, Sw. Mellisuga longicauda, G.R. Gr. M. platura, Steph.

S. America (Guiana). 
1238. ? Trochilus ligonicaudus.

Trochilus (Ovreatus) ligonicaudus, Gould. Mellisuga ligonicauda, G. R. Gr. Discosura ligonicauda, $\operatorname{Pr}$. $B$.

S. America (Brazil).

\author{
k, k. Loddigesia et Loddigiornis, Gould, 1850.
}

1239. Trochilus mirabilis.

Trochilus mirabilis, Lodd. et Bourc. Mellisuga mirabilis, G. R. Gr. \& Mitch. Gen. of B. pl. 37. Loddigesia mirabilis, Gould.

S. America (Peru).

\title{
1, 1. Spathura, Gould, 1850. Steganurus, Reichenb. 1849. \\ Ocreatus, p. Gould, 1846.
}

1240. Trochilus Underwoodir.

B. M.

Ornismya Underwondii, Less. Troch. t. 37. T. ventilabrum, Lath. O. Kieneri, Less. Troch. t. 65. Trochilus (Platurus) Underwoodii, Less. Cynanthus Underwoodii, Jard. Nat. Libr. xv. pl. 22. Mellisuga Underwoodii, G. R. Gr. Spathura Underwoodii, Gould, Monogr. Troch. i. pl. 9.

S. America (New Granada, Venezuela).

1241. Trochilus peruvianus.

Spathura peruviana, Gould, Monogr. Troch. i. pl. 10.

S. America (Peru).

1242. Trochilus AdDe.

B. M.

Trochilus Addæ, Bourc. Trochilus (Ocreatus) rufocaligatus, Gould. Mellisuga rufocaligata, G. R. Gr. Spathura addæ, Pr. B. Gould, Monogr. Troch. i. pl. 11.

S. America (Bolivia). 
m, m. Selasphorus, Sw. 1831.

Les Rubis, Less.

1243. Trochilus ruber.

B. M.

Trochilus ruber, $L$. T. rufus, Gm. T. collaris, Lath. Gen. Syn. pl. 35. Edw. B. pl. 32. Vieill. Ois.dor. t.61,62. T. sitkeusis, Ratke. Orthorhynchus collaris, Cuv. Trochilus (Calliphlox) sasin, Less. Ornismya sasin, Less. Ois. Mouch. t. 66, 67. Suppl. t. 11, 12, 13. Troch. t. 43. Calliphlox sitkensis, Boie. Selasphorus rufus, $S w$. S. ruber, $\operatorname{Pr} . B$. Mellisuga? rubra et M. collaris, Steph. Natr. Libr. xพ. pl. 9. Audub. B. of Amer. pl. 379. Brandt, Icon. Av. Ross. 1. 1, 2.

N.-W. America.

1244. Trochilus anna.

B. MI.

Ornismya anna, Less. Ois. Mouch. t. 47. Suppl.7. Trochilus icterocephalus, Nutt. Trochilus (Calliphlox) anna, Less. Mellisuga anna, G. R. Gr. Selosphorus anna, Pr. B. Nat. Libr. xiv. pl.6. Audub. B. of Amer. pl. 425.

N.-W. America (California).

1245. Trochilus platycercos. Trochilus platycercus, $S w$. Ornismya tricolor, Less. Ois. Mouch. Suppl. t. 14. Troch. t. 60. O. montana, Less. Troch. t. 63. Truchilus (Calliphlox) tricolor et T. (Les Amethystes) moutana, Less. Cynanthus? tricolor, Jard. Nat. Libr. xv. pl. 13. Mellisuga platycerca et M. montana, G. R. Gr. Selasphorus platycercus, $S w$.

Central America (Mexico).

1246. Trochilus Costa.

Ornismya Costæ, Bourc. Ann. Sci. Phys. \&.c. de Lyon, 1840, t.

2. Voy. de la Venus, Ois. t. 2. Mellisuga costæ, G. R. Gr.

Selosphorus costæ, $\operatorname{Pr}$. B.

N.-W. America (California).

1247. Trochilus Jourdani.

Ornismya Jourdani, Bourc. Ann. Sc. Phys. \&-c. de Lyon, 1840, t. 5, 6. Mellisuga Jourdani, G. R. Gr. Calothorax Jourdani, $\operatorname{Pr} . B$.

Trinidad. 
1248. Trochilus Heliodore.

B. M.

Ornismya Heliodore, Bourc. Ann. Sci. Phys. \&c. de Lyon, 1842, t. 15, 16. Mellisuga Heliodori, G. R. Gr. Calothorax Heliodori, $\operatorname{Pr}, B$.

S. America (New Granada).

1249. Trochilus Ros A.

B. M.

Trochilus rosæ, Bourc. \& Muls, Mellisuga rosæ, G. R. Gr. Calothorax Jourdani, p. Pr. B.

S. America (Venezuela).

1250. Trochilus Heloisa.

B. $\mathrm{M}$.

Ornismya Heloisa, DeLattr. \&- Less. Mellisuga Heloise, $G$. R. $G r$.

S. America.

1251. Trochilus Tindal.

Trochilus Tindali, Tschudi. Trochilus (Calothorax) calliope,

Gould. Calothorax calliope, G. R. Gr.

Central America (Mexico).

1252. Trochilus Mulasant.

B. M.

Ornismya Mulsanti, Bourc. Ann. Sc. Phys. \&c. de Lyon, 1842, t. 20. Mellisuga Mulsanti, G. R. Gr. Calothorax Mulsanti, Pr. B.

S. America (Bolivia).

n, n. Heliactin, Boie, 1831.

Heliactinia, Reichenb. 1849.

1253. Trochilus cornutus.

B. $\mathrm{M}$.

Trochilus cornutus, Pr. Max. T.bilophus, Temm. Pl. col. 18, f.3. T. dilophus, Vieill. Heliactin bilophus, Boie. H. cornutus, $\mathrm{Pr}$. B. Orthorhynchus bilophus, Cuv. Trochilus (Heliactin) chrysolophus, Less. Ornismya chrysolopha, Less. Ois. Mouch. t. 7, 8. Suppl. t. 32. Mellisuga cornuta, G, $R$. Gr. M. bilopha, Steph. Nat. Libr. xiv. pl. 21, 22.

S. America (Brazil). 
0, o. Gouldia, $\operatorname{Pr} . B .1850$.

1254. Trochilus LangsdorfFir.

B. $M$.

Trochilus Langsdorffi, Vieill. $P l$. col. 66, f. 1. Ornismya Langsdorffi, Less. Ois. Mouch. t. 26. Suppl. t. 16. Troch. t. 35, 36. Trochilus (Heliactin) Langsdorffi, Less. Colibri hirundinaceus, Spix, Av. Bras. t. 81, f. 2, Heliactin Langsdorffi, Boie. Orthorhynchus Langsdorfii, Cuv. Cynanthus Langsdorffi, Jard. Nat. Libr. xv. pl. 10. Mellisuga Langsdorffii, Steph. Gouldia Langsdorffi, Pr. B.

S. America (Brazil).

1255. Trochilus Conversi.

Trochilus Conversi, Bourc. et Muls. Rev. Zool. 1846, t. 3. Mellisuga Conversi, G. R. Gr. Gouldia conversi, Pr. B.

S. America (New Granada).

1256. Trochilus Popelairi.

Trochilus Popelairi, Dubus, Esquis. Ornith. t. 6. Mellisuga

Popelairi, G. R. Gr. Gouldia Popelairi, Pr. $B$.

S. America (Columbia).

$$
\text { p, p. Tryphæna, Gould, } 1850 .
$$

1257. Trochilus Duponti.

B. M.

Ornismya Dupontii, O.zemes et O. cælestis, Less. Ois. Mouch.

Suppl. t. 1. Trochilus (Les Lucifers) Dupontii, Less. Cynanthus Dupontii, Jard. Nat. Libr. xiv. pl. 26. Mellisuga Dupontii, G. R. Gr. Tryphæna Dupontii, Gould, Monogr. Troch. pl. 14.

Central America (Mexico, Guatimala).

1258. Trochilus amethystinds.

B. M.

Trochilus amethystinus, $G m . \quad P l$. enl. 672, f. 1. (?) T. campestris, Pr. Max.? Omismya amethystina, Less. Ois. Mouch. t. 47. Suppl. t. 20, 21, 22. Trochilus (Calliphlox) amethystinus, Less. Orthorhynchus amethystinus, Cuv. Calliphlox amethystinus, Boie. Mellisuga amethystinus, Steph. Cynanthus amethystinus, Jard. Nat. Libr. xv. pl. 9. Tryphæna amethystina, $\operatorname{Pr}$. $B$.

S. America (Guiana, Brazil). 
1259. Trochilus aMethystordes.

B. $M$.

Ornismya amethystö̈des, Less. Troch. t. 25, 26, 27, 30. 0 . orthura, Less. Troch. t. 28, 29. Trochilus (Calliphlox) amethystoides, Less. T. (Les Rubis) optthura,' Less. Cynanthus amethystoides, Jard. Mellisuga amethrstoïdes et M. orthura, G.R. Gr. Triphæna amethystoïdes, Pr. B. Nat. Libr. xv. pl. 8.

S. America (Brazil).

1260. Trochilds Mitchellit.

Trochilus Mitchellii, Bourc. Mellisuga Mitchellii, G.R. $G r$.

Central America (Mexico).

$$
\text { q, q. Cynanthus, } S w .1827 .
$$

1261. Trochiles COLUBRIS.

B. M.

Trochilus colubris, $L . \quad E d w . B$. pl. 38. Vieill. Ois. dor. t. 31, 32. Wils. Amer. Orn. pl. 10, f. 3, 4. Audub. B. of Amer. pl. 47. Nat. Libr. xiv. pl. 1. T. tomineo, L. Sagra, Voy. de II Isle Cuba, t. 21, f. 1. Ornismya colubris, Less. Ois. Mouch. t. 48, 48*. Troch. t. 1. Trochilus (Calliphlox) colubris, Less. Orthorhynchus colubris, Cuv. Calliphlox colubris, Boie. Cynanthus colubris, $S w$. Mellisuga colubris, Steph.

$\mathrm{N}$. America and W. Indies.

1262. Trochilts Alexandri.

Trochilus Alexandri, Bourc et Muls.

B. M.

Central America (Mexico).

1263. ? Trochilus Swainsoni.

B. M

Trochilus Swainsoni, Less. Troch. t. 66. T. (Glaucis) Swainsoni, Less. Phæthornis Swainsoni, Jard. Nat. Libr. xv. pl. 30 .

S. America (Brazil). 
r, r. Lophornis, Less. 1829.

Bellatrix, Boie, 183 .

1264. Trochilus ornatus.

B. M.

Trochilus ornatus, Bodd. Pl. enl. 640, f. 3. Vieill. Ois, dor. 1. 49, 50,51. T. auratus, $G m$. Ornismya ornata, Less. Ois. Mouch.t. 41. Troch. t. 24. Trochilus (Lophornis) ornatus, Less. Orthorhynchus ornatus, Cuv. Bellatrix ormatus, Boie. Mellisuga ornata, Steph. Lophornis ornata, G. R. Gr. L. auratus, Pr. B. Nat. Libr. xiv. pl. 15, 16. Selasphorus ornatus, $S w$.

S. America (Guiana, Brazil).

1265. Trochilus Regulus.

Ornismya (Lophorinus) Delattrei, I.ess. Mellisuga Delattri, G. R. Gr. Lophornis Delattrii, Pr. B. Trochilus (Lophornis) regulus, Gould. Mellisuga regula, G. R. Gr. Lophornis regulus, Gould.

S. America (Columbia).

1266. Trochilus Helene.

Ornismya Helenæ, Delattr. Mellisuga Helenæ, G. R. Gr. Lophornis Helenæ, Pr. $B$.

Central America (Guatimala).

1267. Trochilus Gouldit.

Ornismya Gouldii, Less. Troch. t. 36. Trochilus (Lophornis) Gouldii, Less. Mellisuga Gouldii, G. R. Gr. Lophoruis Gouldii, $\operatorname{Pr} . B$.

S. America (Brazil).

1268. Trochilus magnificus.

B. M.

Trochilus magnificus, Vieill. $P l$. col. 299, f. 2. T. decorus,

Licht. Ornismya strumaria, Less. Ois. Mouch." t. 42, 43. Colihri Helios, Spix, Av. Bras. t. 82, f. 2, Bellatrix magnificus, Boie. Mellisuga magnifica, Steph. Orthorhynchus magnificus, Cuv. Lophornis magnifica, $\operatorname{Pr} . B$.

S. America (Brazil).

1269. Trochllus REgINA.

Lophornis reginæ, Gould. Mellisuga reginæ, G. R. Gr.

S. America. 
1270. TROCHILUS CHALYBæUS.

B. M.

Trochilus chalybæus, Vieill. T. festivus, Licht. Bellatrix festivus, Boie. Ornismya Vieillotii, Less. Ois. Mouch. t. 64. Troch. t. 8, 9, 10, 11 . O. Audenetii, Less. Col. Suppl.t. 2. Trochilus (Lophornis) Vieillotii et T. (L.) Audenetii, Less. Orthorhynchus chalybæus, Cuv Mellisuga chalybæa, Steph. M. Audenetii, G.R. Gr. Lophornis chalybæa er L. Audenetii, Pr. B. Colibri mystax, Spix, Av. Bras. t. 82, f. 3 . Nat. Libr. xiv. pl. 18. Selasphorus chalybeus, $S w$.

S. America (Brazil).

\section{s, s. Sephanoïdes, Less. 1829. \\ Eustephanus, Reichenb. 1849.}

1271. TrochILUS GaLERITUS.

B. M.

Trochilus galeritus, Mol. T. flammifrons, Lyell. T. sephanoïdes, Hartl. Orthorhynchus sephanoïdes, Less. Voy. de la Coqu. t. 31, f. 2. Mellisuga Kingii, Vigors. M. galerita, G. R. Gr. Orthorhynchus sephanoides, Cuv. Trochilus (Sephanoïdes) ___ Less. Ornismya sephanoides, Less. Ois. Mouch. t. 14. Col. Suppl. t. 5. Sephanoïdes Kingii, Less. S. galerita, Pr. B. Nat. Libr. xiv. pl. 12.

S. America (Chili).

1272. Trochilus Stokesir.

B. M.

Trochilus Stokesii, King. Nat. Libr. xv. pl. 5. Ornismya Stokesii, Less. Troch. t. 50. Trochilus (Les Sephanoides) Stokesii, Less. Mellisuga Stokesii, G.R. Gr. Sephanoïdes Stokesii, $\operatorname{Pr}$. B.

Island of Juan Fernandez.

1273. Trochiles Fernandensis.

Trochilus Fernandensis, King. Ornismya cinnamomea, Gerv. Mag. de Zool. 1835. Ois. t. 43. O. Rohinson, Less. Mellisuga fernandensis, G. R. Gr. Sephanoïdes fernandensis, Pr. B.

Island of Juan Fernandez. 
t, t. Chrysolampis, Boie, 1831.

1274. Trochilus moschitus.

B. $M$.

Trochilus moschitus ( $P l$. enl. 227, f. 2). T. elatus ( $P l$. enl. $640, f, 1)$ et T. pegasus, $L$. T. guianensis, T. striatus, T. leucogaster ( $P l$. enl. 672, f. 3 ) et T. carbunculus, $\mathrm{Gm}$. T. hypophæus, Lath. Vieill. Ois. dor. t. 29, 30, 46, 55, 56, 28, 54. Nat. Libr. xiv. pl.11. Orthorhynchus moschitus, Cuv. Trochilus (Chrysolampis) moschitus, Less. Mellisuga moschita, Steph. Ornismya moschita, Less. Ois. Mouch. t. 52, 53, 54. Troch. t. 15. Chrysolampis moschitus, C. elatus, C. guanensis et C. carbunculus, Boie. Sw. B. of Braz. pl. 30.

S. America (Guiana).

u, u. Orthorhynchus, Cuv.

1275. Trochilus cristatus.

B. M.

Trochilus cristatus, L. Sw. B. of Braz. pl. 21. T. puniceus, Gm.? T. pileatus, Lath. Edw. B. pl. 37. Vieill. Ois. dor. t. 63? Mellisuga cristata et M. pileata, Steph. Ornismya cristata, Less. Troch. t. 4. Orthorhynchus cristatus et O. pileatus, Cuv.

Islands of Trinidad, Martinique, and Barbadoes.

1276. Trochilus Exilis.

B. M.

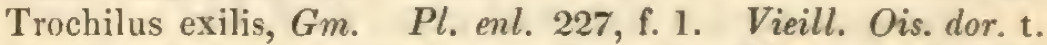
31, 32. Ornismya cristata, p., Less. Ois. Mouch. t. 31, 32. Bellatrix exilis, Boie. Mellisuga exilis, G. R. Gr. Orthorhynchus chloroliphus, $\mathrm{Pr} . \mathrm{B}$.

West Indies.

1277. Trochilus Vietllotil.

B. M.

Trochilus niger, L.P Briss. Orn. iii. t. 36, f. 8 ? T. Vieillotii, Shaw. Vieill. Ois. dor. t. 53. T. humilis, Gosse, B. of Jam. pl. 21. Hylocharis niger, G. R. Gr.?

W. Indies (St. Domingo, Jamaica).

1278. ? Trochilus melanolophus.

Trochilus melanolophus, Vieill. Orthorhynchus melanolophus, $\operatorname{Pr} . B$. 
v, v. Cephallepis, Lodd. 1831.

1279. Trochilus Delalandir.

B. M.

Trochilus Delalandii, et T. versicolor, Vieill. $P l$. col. 18, f. 1, 2. Sw. B. of Braz. pl. 22. Nat. Libr. xiv. pl. 1, xv. pl. 7. Erman, Verz, von Thier, \&c. t. 2. Ornismya Delalandii, Less. Ois. Mouch. t. 23, 24. Suppl.t. 19. Troch. t. 41. Trochilus (Smaragdites) Delalandii, Less. Orthorhynchus Lalandii, Cuv. Mellisuga Delalandii, Steph. Cephallepis Lalandei, Lodd. Hylocharis versicolor, G. R. Gr.

S. America (Brazil).

1280. Trochilus Loddigesi.

Trochilus Loddigesi, Gould. Nat. Libr, xv. t. 6. T. opisthocomus, Erman, Verz. von Thier. t. 2, f. 3. Ornismya Loddigesi, Less. Troch. t. 51. Trochilus (Smaragdites) Loddigesi, Less. Cephallepis Loddigesii, Lodd.

S. America (Brazil).

$$
\begin{gathered}
\text { w, w. Patagona, G. R. Gr. } 1840 . \\
\text { Patagones, Less. } 1829 .
\end{gathered}
$$

1281. Trochilus gigas.

B. M.

Trochilus gigas, Vieill. Orthorhynchus gigas, $C u v$. Trochilus (Les Patagons) tristis, Less. Ornismya tristis, Less. Ois. Mouch. t. 3. Cynanthus gigas, Jard. Nat. Libr. xv. pl. 3. Patagona gigas et Hylocharis gigas, G. R. Gr r.

S. America (Chili).

$$
\begin{aligned}
& \text { x, x. Eriopus, Gould, } 1847 . \\
& \text { Eriocnemus, Reichenb. } 1849 . \\
& \text { Vestipedes, Less. }
\end{aligned}
$$

1282. Trochilus vestitus."

B. M. Ornismya vestita, Longuem. O. glomata, Less. Trochilus uropygialis, Fras. Eriopus vestitus, Gould. Hylocharis vestitus, G.R. Gr. Trochilus vestitus, Hartl.

S. America (New Granada).

1283. Trochilus cupreotentris.

Trochilus cupreoventris, Fras. Ornismya maniculata, Less. O. vestita, \&, Less. Eriopus cupreoventris, Gould. Hylocharis cupreoventris, $G . R$. Gr.

S. America (New Granada). 
1284. Trochilus Simplex.

Eriopus simplex, Gould, Proc. Z. S. 1849, p. 96.

S. America (New Granada).

1285. Trochilus ldeiani.

B. M.

Trochilus luciani, Bourc. Eriopus luciani, $\operatorname{Pr}, B$. Hylocharis luciani, G. R. Gr.

S. America.

1286. Trochilus mosquera.

Trochilus mosquera, Bourc. \& Delattr. Eriopus mosquera, Gould. Hylocharis mosquera, G. R. Gr.

S. America (New Granada).

1287. 'Trochilus ALINe.

B. M.

Ornismya aline, Bourc. Eriopus aline, Gould. Hylocbaris aline, G. R. Gr.

S. America (Columbia).

1288. Trochilus Derbyi.

Trochilus Derbyi, Bourc. \& Delattr. Eriopus Derbyi, Gould.

Hylocharis Derbyi, G. R. Gr.

S. America (New Granada).

1289. Trochilus aUreliz.

Trochilus aureliæ, Bourc. \& Muls. Hylocharis aureliæ, G. R.

Gr. Eriopus? aureliæ, $\operatorname{Pr}, B$.

S. America (New Granada).

1290. Trochilus Isaacsoni.

Ornismya Isaacsoni, Parduz. Hylocharis Isaacsoni, G. R. Gr. Eriopus? Isaacsoni, $\mathrm{Pr}$. B.

S. America (New Granada).

1291. Trochilus Godini.

Trochilus Godini, Bourc.

S. America (Ecuador). 
y, y. Avocettes, Less. 1829. Avocettula, Reichenl. 1849. Avocettinus, Pr. B. 1850.

1292. Trochilus Recurvirostris.

B. M.

Trochilus recurvirostris, Sw. Zool. Illustr. pl. 105. Nat. Libr. xiv. pl, 3. Ornismya recurvirostris, Less. Ois. Mouch. Suppl. t. 34. O. avocetta, Less. Col. Suppl. t. 24. Troch. t. 23. Trochilus (Les Avocettes) recurvirostris et T. (Les A.) a rocetta, Less. Mellisuga? recurvirostris, Steph. Hylocharis recurvirostris et H. avocetta, $G . R$. Grr. Campylopterus recurvirostris, Sw. Avocettinus recurvirostris et A. Lessoni, $\operatorname{Pr}$. B.

S. America (Guiana).

1293. Trochilus eurypterus.

Trochilus eurypterus, Lodd. Polytmus eurypterus, G. R. Gr.

S. America (New Granada).

1294. Trochilus Georgin z.

Trochilus Georginæ, Bourc. Polytmus Georginæ, G. R. Gr.

S. America (New Granada).

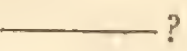

1295. TROCHILUS OURISSIA.

Trochilus ourissia, $L . \quad P l$. enl. 227, f. 3. T. maugæus, Vieill. T. maugeana, Shaw. T. tabaci, Gm. T. tabagensis, Lath. Ois. dor. t. 37, 38. Ornismya Maugei, Less. Ois. Mouch. t. 68, 69. Trochilus (Mellisuga) Maugei, Less. Hylocharis ourissia, G. R. Gr. Thaumatias ourissia, Pr. B. Orthorhynchus Maugeanus, Cuv. Mellisuga Maugeana et M. surinamensis, p., Steph.

S. America (Guiana); W. Indies (Porto Rico).

1296. Trochilus Canivetil.

B. $M$.

Ornismya Caniveti, Less. Ois. Mouch. Suppl. t. 37, 38. Trochilus (Mellisuga) Canivetii, Less. Cynanthus Canivetii, Jard. Hylocharis Caniretii, G.R.Gr. Thanmatias Canivetii, $\operatorname{Pr} . B$.

S. America (Brazil). 
1297. Trochilus ELEgANS.

B. M.

Trochilus elegans, Vieill. Ois. dor. t. 14. Ornismya Swainsoni, Less. Ois. Mouch. t. 70. Hylocharis elegans, G. R. Gr. Lampornis elegans, $\operatorname{Pr} . B$. Polytmus elegans, Vieill.

S. America (Brazil).

1298. Trochilds Ricordi.

B. M.

Ornismva Ricordii, Gerv. Mag. de Zool. 1835. Ois. t. 41, 42. O. Parzudaki, Less. Hylocharis Ricordii, G. R. Gr. Orthorhynchus Ricordii, Sagra, Voy. de l'Ile de Cuba, t. 21, f. 2 . Trochilus Ricordii, $\mathrm{Pr} . \mathrm{B}$.

West Indies (Cúba).

1299. Trochilus Cathenine.

Ornismya Catherinz, Salé, Rev. et Mag. de Zool. 1849, p. 499.

W. Indies (St. Domingo).

1300. Trochilus JULiæ.

B. II.

Ornismya Juliæ, Bourc. Ann. Sci. Phys. \&c. Lyon, 1843, t. 21. O. feliciana, Less. Hylocharis Juliæ et H. feliciana, $G$. R. Gr.

S. America (New Granada).

$$
\text { z, z. Saucerottia, Pr. B. } 1850 .
$$

1301. Trochilus Sadcerottit.

Trochilus Saucerottii, Bourc. \& Muls. Polytmus Saucerottei, G. R. Gr. Saucerottia typica, $\operatorname{Pr} . B$.

S. America (New Granada).

1302. Trochilus Goudotr.

B. M. Trochilus Goudoti, Bourc. Polytmus Goudoti, G. R. Gr. Saucerottia Goudoti, Pr. B.

S. America (New Granada).

1303. Trochiles erythonotes,

B. $\mathrm{M}$. Ornismya erythronotos, Less. Ois. Mouch. t. 61. Trochilus (Les Amaziles) erythronotos, Less. Cynanthus erythronotos, Jard. Polytmus erythronotos, G.R.Gr. Saucerottia erythronota, Pr. B.

S. America (Brazil?); Central America (Mexico). 
1304. Trochilus Devillei.

Trochilus Devillej, Bourc. \& Muls.

Central America (Guatimala).

1305. Trochilus Lessoni.

Ornismya Lessonii, Delattr. Hylocharis Lessoni, G. R. Gr.

S. America.

1306. Trochilus caligatus.

Trochilus (G. R. Gr. Saucerottia caligata, $\operatorname{Pr} . B$.

S. America (New Granada).

a, a, a. Amazilis, Less. 1829.

Amazilia, Reichenb. 1849.

Amazilius, Pr. B. 1850.

1307. TrochILUS AMIZILI.

B. M.

Orthorhynchus amazilii, Less. Voy. de la Coqu. Ois. t. 31, f. 3.

Ornismya amazili et Trochilus (Les Amazilis) amazili, Less.

Ois. Mouch. t. 12, 13. Trochilus latirostris, Sw. Orthorhvnchus amisili, Cuv. Trochilıs (Lampornis) amazilia, Tschudi. Phætornis? amazili, Jard. Amiziles latirostris et Polytmus amazili, G. R. Gr. Amizilia latirostris, Pr. $B$.

S. America (Peru).

1308. Trochilus Norrissi.

Trochilus Norrissii, Bourc. Polytmus Norrissii, G. R. Gr.

Amazilius Norrissii, $\operatorname{Pr}$. $B$.

S. America (Ecuador).

1309. Trochilus Riefferi.

B. M.

Trochilus Riefferi, Bourc. Polytmus Riefferi, G. R. Gr. Amazilius Riefferi, $\operatorname{Pr}$. $B$.

S. America (New Granada and Gorgona Island).

1310. ? Trochilus PhäBe.

Ornismya Phœbe, Less. \& Delattr. Mellisuga Phœbe, G. R. Gr.

S. America (Peru). 
1311. TrochILUS AgLAite.

Trochilus aglaiæ, Bourc. \& Muls. Polytmus aglaiæ, G. R. Gr. Amazilius aglaiæ, $\operatorname{Pr}$. B.

S. America ?)

1312. Trochilus corallirostris.

B. M.

Trochilus corallirostris, Bourc. \& Muls. Polytmus corallirostris, G.R. Gr. Amazilius corallirostris, $\operatorname{Pr}$. B.

Central America (Guatimala).

1313. Trochilus Arsinoe.

Ornismya Arsinoe, Less. Ois. Mouch. Suppl. t. 28. Trochilus (Les Amaziles) Arsinoe, Less. Cynanthus arsinöe, Jard. Polytmus arsinöe, G. R. Gr. Amazilius arsinöe, Pr. B.

Central America (Mexico).

1314. Trochilus Sophie.

Trochilus Sophiæ, Boure. Polytmus Sophiæ, G. R. Gr. Amazilius sophiæ, $P r, B$.

S. America (New Granada).

1315. Trochilus EdWard. B. M. Trochilus Edward, Delattr. \& Bourc. Polytmus Edwardsii, $G$. $R$. Gr. Amazilius Edwardsii, $P r, B$.

Central America (Panama).

1316. Trochilus Dumerilit.

Ornismya Dumerilii, Less. Ois. Mouch. Suppl. t. $36 . \quad$ Polytmus Dumerilii, G. R. Gr. Amazilius Dumerilii, Pr. B.

S. America (Chili?).

$$
\begin{aligned}
& \text { b, b, b. Chrysures, Less. } 1829 . \\
& \text { Chrysurontia, Pr. B. } 1850 .
\end{aligned}
$$

1317. Trochilus GNone.

B. $M$.

Ornismya œnone, Less. Col. Suppl. t. 30. Trochilus (Les Chrysures) œnone, Less. Cynanthus cenone, Jard. Polytmus œnone, G. R. Gr. Chrysuronia œnone, $\operatorname{Pr}$. B.

Island of Trinidad. 
1318. Trochilus Josephin .

Trochilus Josephinæ, Bourc. \& Muls.

America (- ? )

1319. Trochilus chrysurus.

Trochilus chrysurus, Shaw. Ornismya chrysura, Less. Ois. Mouch. Suppl. t. 4. Trochilus (Les Chrysures) chrysura, Less. Phæthornis? chrysurus, Jard. Polytmus chrysurus, G. R. Gr . Chrysuronia chrysura, $\operatorname{Pr}$. $B$.

S. America (Brazil).

1320. Trochilus ELICLE.

Trochilus eliciæ, Bourc. \& Muls. Polytmus eliciæ, G. R. Gr. Chrysuronia eliciæ, $\operatorname{Pr} . B$.

S. America (Brazil).

1321. Trochilus phaeopygus.

Trochilus phaeopygus, Licht. T. (Lampornis) phaeopygus, Tschudi. Polytmus phaeopyga, G. R. Gr. Chrysuronia phaeopygus, $\operatorname{Pr}$. $B$.

S. America (Peru).

$$
\begin{aligned}
& \text { c, c, c. Polytmus, Less. } 1832 . \\
& \text { Trochilus, G. R. Gr. } 1840 .
\end{aligned}
$$

1322. Trochilus polytmus.

B. M.

Trochilus polytmus, L. Edw. B. pl. 34. Vieill. Ois. dor. t. 67. Trochilus (Les Polytmus) cephalatra, Less. Ornismya cephalatra, Less. Ois. Mouch. t. 17. Mellisuga polytmus, Boie. $\quad$ Cynanthus polytmus, Jard. Nat. Libr. xv. pl. 21. Polytmus atricapillus, Vieill. P. cephalatra, Pr.B. Gosse, B. of Jam. pl. 19, 20.

W. Indies (Jamrica).

$$
\text { d, d, d. Hylocharis, Boie, } 1831 .
$$

1323. Trochilus sappririnus.

B. M.

Trochilus sapphirinus, Gm. Vieill. Ois. dor. t. 35, 58. T. latirostris, $\mathrm{Pr}$. Max.? Ornismya sapphirina, Less. Ois. Mouch. t. 55, 57. Troch. t. 15. Trochilus (Hylocharis) sapphirinus, Less. Hylocharis sapphirinus, Boie. Mellisuga sapphirina, Steph. Cynanthus saphurinus, Jard. Nat. Libr. xiv. pl. 28.

S. America (Guiana, Brazil). 
1324. Trochitus Lacteus.

B. M.

Ornismya lactea, Less. Ois. Mouch. t. 56. Trochilus (Hylocharis) lacteus, Less. Hylocharis lactea, G. R. Gr.

S. America (Brazil).

1325. Trochilus Pucherani.

Trochilus Pucherani, Bourc. \& Muls.

S. America (Brazil).

1326. Trochilus cyaneus.

B. M.

Trochilus cyaneus, Vieill. Ornismya cyanea, Less. Ois. Mouch. t. 71. Suppl. t. 23. Troch. t. 22. Trochilus (Les Bleuets) cyaneus, Less. Hylocharis cyaneus, Boie. Thaumatias cyaneus, Pr. B. Nat. Libr. xiv. pl. 7.

S. America (Brazil).

1327. Trochilus Doubleday.

Trochilus Doubledayi, Bourc. Hylocharis Doubledayii, G. R. $G r$. Thaumatias Doubledayi, $P r . B$.

S. America (Brazil ?).

1328. Trochilus Duxchassaingui.

Trochilus Duxchassaingui, Bourc.

S. America (Ecuador).

1329. Trochilus Felicle.

Ornismya Feliciæ, Less.

S. America (Brazil).

1330. Trochilus Cardeleds.

B. M.

Trochilus cæruleus, Vieill. Ois. dor, t. 40. Ornismya Audebertii, Less. Ois. Mouch. t. 51. Trochilus (Hylocharis) Audebertii, Less. Cynanthus? cæruleus, Jard. Hylocharis cærulea, G.R. Gr. Thaumatias cærulea, $\operatorname{Pr}, B$.

S. America (Guiana).

1331. Trochilus Pheton.

Trochilus Phæton, Bourc. \& Muls.

America (- ?). 
1332. Trochilus Bicolor.

B. M.

Trochilus bicolor, Gm. Ois. dor, t. 36, T. smaragdo-sapphirinus et T. lucidus, Shaw. T. azureus, Licht. T. Splendidus, Vieill. Ornismya bicolor, Less. Ois. Mouch. t. 49, 50. Troch. t. 16. Trochilus (Hylocharis) bicolor, Less. Hylocharis bicolor, G. R. Gr. Thaumatias bicolor, $\operatorname{Pr} . B$. Mellisuga smaragdo-sapphirina et M. lucida, Steph.

S. America (Guiana).

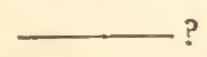

1333. Trochilus Grayi.

B. M.

Trochilus Grayi, Delattr. \& Bourc. Hylocharis Grayii, G. R. Gr.

S. America (New Granada).

1334. Trochilus simila.

B. M.

Trochilus simila, Bourc. Hylocharis simila, G. R. Gr.

S. America (Bolivia).

1335. Trochilus LEucotis.

B. M.

Trochilus leucotis, Vieill. T. leucocrotaphus, Shaw. T. melanotis, $S w$. T. cuculliger, Licht.? Ornismya Arsennii, Less. Ois. Mouch. t. 9. Suppl. t. 27. Hylocharis leucotis, G. R. Gr. Thaumatias leucotis, $\operatorname{Pr} . B$.

S. America (Mexico, Brazil).

1336. Trochilus Guimet.

B. M.

Trochilus Guimeti, Bourc. \& Muls. Ann. Sci. Phys. \&॰. de Lyon, 1843, t. 2. Hylocharis Guimeti, G. R. Gr.

S. America (Venezuela).

B. M

1337. Trochilus prasinus.

Trochilus viridissimus, var. Lath. T. prasinus, Jard. Nat. Libr. xiv. pl. 8. Ornismya prasina, Less. Ois. Mouch. t. 65. Trochilus (Hylocharis) prasinus, Less. Hylocharis prasina, G. R. Gr.

S. America (Brazil). 
1338. Trochilus Cyanogenys.

B. M. Trochilus cyanogenys, $P r . M a x . \quad$ T. viridissimus, var. $\beta$., Lath. Edw, B. pl. 360, f. 1 . Trochilus (Hylocharis) Wiedii, Less. Ornismya Wiedii, Less. Ois. Mouch. Suppl. t. 26. Hylocharis cyanogenys, G. R. Gr. Saucerottia cyanogenys, $\operatorname{Pr}$. B.

S. America (Brazil).

1339. TROCHILUS VIRIDIG ASTER.

B. M. Trochilus viridigaster, Bourc. Hylocharis viridigaster, $G$. R. $G r$.

S. America (New Granada).

1340. Trochildus Maria.

Trochilus mariæ, Bourc. \& Muls. Hylocharis mariæ, G. $R$. $G r$.

S. America (Venezuela).

1341. Trochilus chrysogaster.

B. M.

Trochilus chrysogaster, Bourc. Hylocharis chrysogaster, $G$. R. Gr.

S. America (New Granada).

1342. Trochilus angustipennis.

Trochilus angustipenuis, Fras. Hylocharis angustipennis, $G$. R. Gr.

S. America (Sta Fè de Bogota).

1343. Trochilus Portmann. B. M. Ornismya Portmanni, Boure. Hylocharis Portmanni, G. R. Gr.

S. America (Columbia).

1344. Trochilus alice.

Trochilus alice, Bourc. \& Muls.

S. America (Venezuela).

1345. Trochilus atala.

Trochilus atala, Less. Troch. t. 42. Hylocharis atala, G. R. $G r$. Saucerottia atala, $\operatorname{Pr} . B$.

S. America (Brazil). 
1346. Trochilus mellisugus.

Trochilus mellisugus, $L$. $P l$. enl. 276. f. 3 . Orthorhynchus mellisugus, Cuv. Mellisuga concinna, Steph. Polytmus mellisugus, G. R. Gr. Thaumatias mellisugus, $\boldsymbol{P r}$. B. Ornismya alhirostris, p. Less.

S. America (Guiana).

1347. ? Trochilus MinULLUS.

Trochilus minnllus, Vieill. Ornismya minima, Less. Ois. Mouch.t. 79. Polytmus minullus, G. R. Gr.

W. Indies (St. Domingo).

$$
\text { e, e, e. Augastes, Gould, } 1850 .
$$

1348. Trochilus SUPERBUS.

B. M.

Trochilus superhus, Vieill. T. scutatus, Natt. $P l . c o l .299$, f. 3. Ornismya Nattereri, Less. Ois. Mouch. t. 16. Trochilus (Les Coquets) Nattereri, Less. Orthorhynchus scutatus, Cuv. Mellisuga scutata, Steph. Hylocharis superba, G. R. Gr. Augastes scutata, Gould. A. superba, Pr. B. Nat. Libr. xiv. pl. 14.

S. America (Brazil).

1349. Trochilus Lumachellus.

B. M. Ornismya lumachella, Less. Hylocharis lumachella, G. $R$. Gr. Augastes lumachella, Gould.

S. America (Brazil).

$$
\text { f, f, f. Heliothrix, Boie, } 1831 .
$$

1350. Trochilus auritus.

Trochilus auritus, Gm. Vieill Ois dor t 25, 26. B. M. Trochlus aurits, Gm. Vieill. Ois. dor. 1.25, 26. Ornithorhynchus auritus, Cuv. Trochilus (Les Jacobines) auritus, Less. Ornismya aurita et $\mathrm{O}$. nigrotis, Less. Ois. Mouch. t. 10, 11. Heliothryx auritus, Boie. Mellisuga aurita, Steph. Sw. B. of Braz. pl. 29.

S. America (Guiana, Brazil).

1351. Trochilus adriculatus. B. M. Ornismya Poncheti, Less. Trochilus auriculatus, Licht. Erman, Verz. von Thier. und Pflang. t. 2, f. 1, 2. Heliothrix Poucheti, $\operatorname{Pr} . B$. H. auriculatus, G. R. Gr.

S. Ameriea (Brazil). 
1352. Trochilus Barrotil.

Trochilus Barrotii, Bourc. Ann. Sci. Phys. \&c. de Lyon, 1843, t. 4. Heliothrix Barrotii, G. R. Gr.

S. America (New Granada).

1353. Trochilus Geoffroyil.

B. M.

Trochilus Geoffroyii, Bourc. \& Muls. Ann. Sci. Phys. \&c. de Lyon, 1843, t. 3. Petasophora? Geoffroyi, Gould. Polytmus Geoffroyi, G. R. Gr. Colibri Geoffroyi, Pr. B.

S. America (New Granadia).

1354. ? Trochilus Azaræ.

Trochilus Azaræ, Vieill. Hylocharis Azaræ, G. R. Gr.

S. America (Paraguay).

1355. ? Trochilus cinnamomeus.

Ornismya cinnamomea, Less. Polytmus cinnamomeus, G. $R$. $G r$.

Central America (Mexico, Guatimala).

1356. ?? Trochilus Minimus.

Truchilus minimus, $L$. Briss. Orn. iii. t. 36, f. 1. Pl. enl. 276, f. 1. Edw. B. pl. 105. Shaw, Nat. Misc. pl. 489. Vieill. Ois. dor. t. 64. Mellisuga minima, Steph.

S. America.

1357. ?? Trochilus Gibsoni.

Trochilus Gibsoni, Lodd. Mellisuga Gibsoni, G. R. Gr.

America ?)

1358. ??'Trochilus MULTICOLOR.

Trochilus multicolor, Gm. Lath. Gen. Syn. Suppl. pl. 111. Vieill. Ois. dor. t. 69. Nat. Misc. pl. 81. Less. Ois. Mouch. t. 72. Trochilus (Glaucis) multicolor, Less. Polytmus? multicolor, G. R. Gr.

America ?? (—? ). 


\section{MELIPHAGID Æ.}

Meliphagidx, Vigors, $1825 ;$ Sw. $1831-37 ;$ G. R. Gr. $1840-$ 41-46; Pr. B. 1849-50;Bl. 1849.

Trochilidæ, p. 1831 ; Maluridæ, p. $1850 ; \operatorname{Pr} . B$.

Pycnonotidæ, p. Bl. 1849.

Gymnopides, p.; Meliphagides, Sundev. 1835.

Menurinæ, p. Gould, 1848.

\section{i. Myzomeline.}

Myzumelinæ, G. R. Gr. 1840-41-46; Cab. 1847; Pr. B. 1849. Meliphaginæ, p. $B l .1849 ;$ Pr. B. 1850.

\section{Myzomela.}

Myzomela, Vig.\& Horsf. 1826.

Phylidonyris, Less. 1831.

1359. Mrzomela Sayguinolentá.

B. M.

Certhia sanguinolenta, C. dibapha et C. erythropygia, Lath. C. australasiæ, Leach, Zool. Misc. pl. 11. Meliphaga sanguinea, Steph. M. cardinalis, Vig. \&. Horsf. M. sanguinolenta, Temm. Melithreptus sanguinolentus, M. erythropterus, et M. dibaphus, Vieill. Myzomela sanguinolenta, Gould, B. of Austr. iv. pl. 63. M. dibapha, G. R. Gr.

New South Wales and N. Australia.

1360. MYzomela erythrocephala.

Myzomela erythrocephala, Gould, B. of Austr. iv. pl. 64.

N. Australia.

1361. Myzomela pectoralis.

Myzomela pectoralis, Gould, B. of Austr. iv. pl. 65.

N. Australia.

1362. Myzomela obscura.

B. M.

Myzomela obscura, Gould, B. of Austr. iv. pl. 67.

N. Australia. 
1363. Myzomela nigra.

B. MI.

Myzomela nigra, Gould, B. of Austr. iv. pl. 66.

Australia (except Van Diemen's Land).

1364. Mrzomela Lafargei.

Myzomela - Homb. \&. Jacq. Voy. au Pole Sud, Ois. t. 22 , f. 5 .

1365. Mrzomela solitaria.

Myzomela —, Homb. \& Jacq. Voy. au Pole Sud, t. 22, f. 6 .

1366. Myzomela chermesina.

B. M.

Myzomela chermesina, G. R. Gr. \& Mitch. Gen. of B. pl. 38.

$\longrightarrow$

1367. Myzomela cardinalis.

B. M.

Certhia cardinalis, Gm. Lath. Gen. Syn. t. 33, f. 2 . Nectarinia cardinalis, Steph. Melithreptus cardinalis, Vieill. Meliphaga cardinalis, p. Vig. \&. Horsf. Myzomela nigriventris, Peale, U. S. Expl. Exped. viii. 150, pl. 41, f. 1.

New Hebrides (Island of Tanna) and Navigators' Islands.

1368. Mrzomela rubratra.

Cinnyris rubrater, less. Kittl. Kupf. t. 8, f. 1.

Islands of the S. Sea.

1369. Myzomela Vieilloti.

Certhia cardinalis, Vieill. Ois. dor. t. 58. Shaw, Gen. Zool. viii. pl. 35 , low. f. ठ .

Island of the S. Sea?

1370. Mrzomela jugularis.

Myzomela jugularis, Peale, U. S. Expl. Exped. viii. p. 150, pl. 41, f. 2.

Feejee Islands. 
1371. Myzomela vulnerata.

Myzomela vulnerata, Müll. Verh. Nat. Gesch. Nederl. t. 10, f. 3,4 .

Timor.

1372. Mrzomera Botei.

Myzomela Boiei, Mill. Verh. Nat. Gesch. Nederl. t. 10, f. $1,2$.

Banda.

1373. ? Myzomela guttata.

Certhia guttata, Vieill. Ois. dor. t. 59. Meliphaga guttata, Temm. Myzomela guttata, G. R. Gr.

New Holland (?)

\section{Acanthorhynchus.}

Acanthorhynchus, Gould, 1837.

Leptoglossus, $S w .1837$.

1374. Acanthorhynchus tenuirostris.

B. M.

Certhia tenuirostris, Lath. C. cucullata et C. semitcrquata, Shaw. Melithreptus tenuirostris, M. cucullatus et M. collaris, Vieill. Ois.dor. t. 60, 56. Meliphaga cucullata, Steph. M. tenuirostris, Vig. \&. Horsf. Philemon cucullatus, Vieill. Philedon cucullatus, $C u v$. Acanthorhynchus tenuirostris et A. dubius, Gould, B. of Austr. iv. pl. 61. Leptoglossus cucullatus, $S w$. Nectarinia semitorquata, $C u v$. Furnarius semitorquatus, Steph.

New South Wales and Van Diemen's Land.

1375. ACANThorhyychus superciltosus. B. $M$. Acanthorhynchus superciliosus, Gould, B. of Austr. iv. pl. 62.

IV. Australia. 


\section{GLyciphila.}

Glyciphila, Sw. 1837.

1376. Glyciphila melanops.

B. II.

Certhia melanops, Lath. Vieill. Ois. dor. t. 86. C. mellivora, Shaw. Philedon mellivora, Cuv. P. rubrifrons, Less. Meliphaga fulvifrons et M. melanops, Vig. \& Horsf. M. albirentris, Steph. Melithreptus melanops, Vieill. Glyciphila fulvifrons, $S w$. G. melanops, G. R. Gr. Gould, B. of Austr. iv. pl. 28.

Australia (except N. Australia).

1377. Glyciphila albifrons.

B. M.

Glyciphila albifrons, Gould, B. of Austr. iv. pl. 29.

New South Wales; S. and W. Australia.

1378. Glyciphila fasciata.

B. M.

Glyciphila fasciata, Gould, B. of Austr. ir.pl. 30.

N. Australia.

1379. GLYCIPHILA OCULARIS.

B. 11 . Glyciphila ocularis et G. subocularis, Gould, B. of Austr. iv. pl. 31 .

Australia (except Van Diemen's Land).

1380. ? Gliteiphila agilis.

Certhia agilis, Lath. Melithreptus agilis, Vieill. Glyciphila agilis, G. R. Gr.

Australia.

1381. ? Glyciphila aurita.

Merops aurita, Lath. Philemon auritus, Vieill. Glyciphila aurita, G. R. Gr.

Australia.

1382. ? GLYCiPHILA FUSCA.

Certhia fusca, Gm. Vieill. Ois. dor. t. 65. Glyciphila fusca, G. R. Gr. Tichodroma? fusca, Cur.

Islands of the S. Sea? 


\section{Entomophila.}

i. Entomophila, Gould, 1837.

1383. Entomophila Picta.

B. M.

Entomophila picta, Gould, B. of Austr. iv. pl. 50.

New South Wales.

1384. ENTomophila albogularis.

B. M.

Entomophila albogularis, Gould, B. of Austr. iv. pl. 51.

N. Australia.

1385. Entomophila rufigularis.

Entomophila rufigularis, Gould, B. of Austr. iv. pl. 52.

N. Australia.

b. Melicophila, Gould, 1844 .

1386. Entomophila picata.

B. M.

Melicophaga picata, Gould, B. of Austr. iv. pl.49. Entomoplila picata, $G, R$. Gr.

S. and W. Australia.

\section{ii. Meliphagine.}

Meliphaginæ, G. R. Gr. 1840-41-46; Cab. 1847; Pr. B. 1849 ; Bl. 1849.

Brachypodinæ, Sw. 1837.

Phyllorninæ, Bl. $1849-50$.

Phyllornithinæ, p. Cab. 1847.

Timaliinæ, p. $\operatorname{Pr}, B .1850$.

1. Meliphaga.

a. Meliphaga, Lew. 1808.

Zanthomyza, $S w .1837$.

Xanthomyza, Strickl. 1841. 
1387. Meliphaga phrygia.

B. M.

Merops phrygius, Lath. Shaw, Zool. N. Holl. pl. 4. Meliphaga phrygia, Lew. B. of N. Holl. pl. 14. Philemon phrygius, Vieill. Turdus squamatus, Vieill. Levaill. Ois. de $l^{\prime} A f r$. t. 116. Zanthomyza phrygia, Sw. Xanthomyza phrygia, Gould, B. of Austr. iv. pl. 48. Philedon phrygius, Cuv. Anthochæra phrygia, Vig. \&. Horsf.

New South Wales and S. Australia.

\section{b. Ptilotis, Sw. 1837 .}

1388. Meliphaga chrysotis.

B. M.

Certhia chrysotis, Lath. C. xanthotis, Shaw. Vieill. Ois. dor. t. 84. Meliphaga chrvsotis, Lew. B. of N. Holl. pl. 5. G. R. $G r . \quad M$. Lewinii, G. R. Gr. M. xanthotis, Steph. Ptilotis chrysotis, Gould, B. of Austr. iv. pl. 32. P. Lewinii, Sw. Philedon xanthotis, Cuv. Philemon chrysotis, Vieill.

New South Wales.

1389. Meliphaga anologus.

Ptilotis —, Voy. au Pole Sud, t. 17, f. 2.

1390. Meliphaga sonora.

B. M.

Ptilotis sonora, Gould, B. of Austr. iv. pl. 33. Meliphaga sonora, G. R. Gr.

New South Wales; and S. and W. Australia.

1391. Meliphaga versicolor.

Ptilotis versicolor, Gould, B. of Austr. iv. pl. 34. Meliphaga versicolor, G. R. Gr.

N. Australia.

1392. Meliphaga flavigula. B. M. Ptilotis flavigula, Gould, B. of Austr. ir. pl. 35. Meliphaga flavigula, G.R. Gr. Melithreptes flavicollis, Vieill.?

Van Diemen's Land. 
1393. Melithaga levcotis.

B. $\mathbf{M}$.

Turdus lencotis, Lath. Lew. B. of N. Holl.pl. 20. Meliphaga leucotis, Temm. Pl. col. 435. Ptilotis leucotis, Gould, B. of Austr. iv. pl. 36 Philedon leucotis, Cuv. Jard. \& Selby, Illustr. Orn. pl. 35, f. 2. Voy. de l'Astrol. t. 8, f. 1.

New South Wales and W. Australia.

1394. Meliphaga aUricomis.

B. $M$.

Muscicapa auricomis, M. novæ hollandiæ et M. mystacea, Lath. Certhia auriculata, Shaw. C. chrysotis, Don. Nat. Rep. pl. 112. Turdus melanops, Lath. Philedon auricomis, Cuv. Philemon auricomis et P. errthrotis, Vieill. Ois. dor. t. 85. Ptilotis auricomis, Sw. Zool. Illustr. pl. 43. Gould, B. of Austr. iv. pl. 37. P. melanops, G. R. Gr. Meliphaga auricomis, Vig. \& Horsf. M. melanops, Temm.

New South Wales.

1395. Meliphaga cratitia.

Ptilotis cratitia, Gould, B. of Austr. iv. pl. 38. Meliphaga cratitia, G. R. Gr.

S. Australia.

1396. Melifhaga ornata.

B. $M$.

Ptilotis ornata, Gould, B. of Austr. iv. pl. 39. Meliphaga ornata, $G . R . G r$.

W. Australia.

1397. Meliphaga plemula.

Ptilotis plumula, Gould, B. of Austr, iv. pl. 40. Meliphaga plumula, G. R. Gr.

W. Australia.

1398. Meliphaga flavescens.

B. M.

Ptilotis flavescens, Gould, B. of Austr. iv. pl. 41. Meliphaga flavesceus, G.R. Gr.

N. Australia. 
1399. Meliphaga flava.

Ptilotis flava, Gould, B. of Austr. iv. pl. 42. Meliphaga flava, G. R. Gr.

N. Australia.

1400. Meliphaga penicillata.

B. M.

Ptilotis et Meliphaga penicillata, Gould, B. of Austr. iv. pl. 43.

New South Wales and S. Australia.

1401. Meliphaga fusca.

B. M.

Ptilotis et Meliphaga fusca, Gould, B. of Austr. iv. pl. 44.

New South Wales.

1402. Meliphaga chry sops.

B. M.

Sylvia chrysups, Lath. Meliphaga chrysops, Vig. \&orsf. Ptilotis chrysops, Gould. Jard. \& Selby, Illust. Orn. pl. 35, f. 1. B. of Austr. iv. pl. 45.

New South Wales and S. Australia.

1403. Meliphaga unicolor.

B. M. Ptilotis unicolor, Gould, B. of Austr. iv. pl. 46. Meliphaga unicolor, G. R. Gr.

N. Australia.

1404. Meliphaga maculata.

Meliphaga maculata, Temm. $P l$. col. 29, f. 1. Philedon maculatus, Cuv. Ptilotis maculata, Müll.

Timor.

1405. Meltphaga reticulata.

B. M.

Meliphaga reticulata, Temm. $P l$. col. 29, f. 2. Philedon reticulatus, Cuv. Ptilutis reticulata, Müll.

Timor.

1406. Meliphaga limbata.

Ptilotis limbata, Milll.

Timor. 
1407. Meliphaga fumata.

Ptilotis fumata, Muill. Pr. B. Consp. p. 392. Meliphaga concolor, Temm. MSS.

New Guinea.

c. Meliornis, G. R. Gr. 1840.

Meliphaga, Vig. \& Horsf. 1826.

1408. Meliphaga nove hollandie.

Certhia nove hollandim, Lath. M. Vig. \& Horsf. M. Balgonera, Steph. M. barbata, Sw. Gould, B. of Austr. iv. pl.23. Melithreptus novæ hollandiæ, Vieill. Philedon novæ hollandiæ, Cuv. Meliornis novæ hollandiæ, G. R. Gr. Vieill. Ois. dor. t. 57.

New South Wales; S. Australia and Van Diemen's Land.

1409. Meliphaga australasiana.

B. M.

Certhia australasiana, Shaw. Vieill. Ois. dor. t. 55. C. pyrrhopterus, Lath. Meliphaga australasiana, Vig. \& Horsf. M. inornata, Gould, B. of Austr. iv. pl. 27. Melithreptus pyrrhopterus et M. melanoleucus, Vieill. Philedon australasianus, Cuv. Meliornis australasiana, $\operatorname{Pr} . B$.

New South Wales; S. Australia and Van Diemen's Land.

1410. Meliphaga longirostris. B. $M$. Meliphaga longirostris, Gould, B. of Austr. iv. pl. 24. Meliornis longirostris, $\mathrm{Pr} . \mathrm{B}$.

S. Australia.

1411. Meliphaga sericea.

B. M.

Meliphaga sericea et M. sericeola, Gould, B. of Austr. iv. pl. 25. Melithreptus ater, Vieill. Ois. dor, t. 71? Meliornis sericea, $\operatorname{Pr} . B$.

New South Wales.

1412. Meliphaga mistaCalis.

Meliphaga mystacalis, Gould, B. of Austr. iv. pl. 26. Meliornis mystacalis, $\operatorname{Pr} . B$.

W. Australia. 
1413. Meliphaga melaiodera.

Philedon melanodera, Quoy \& Gaim. Voy. de l Astrol.t. 8, f. 1. Meliphaga melanodera, G. R. Gr. Ptilotis melanodera, Less.

S. Australia.

1414. ? Meliphaga pyrrotis.

Ptilotis pyrrotis, Less.

1415. P Meliphaga flavicans.

Melithreptus flavicans, Vieill.

Australia.

1416. ? Meliphaga gilvicapilla.

Melithreptus gilvicapillus, Vieill.

Australia.

d. Creadion, Vieill. 1816.

1417. Meliphaga carunculata.

B. M.

Certhia carunculata, Gmel. Vieill. Ois. dor. t. 69, 70. Meliphaga carunculata, Temm. Creadion musicus, Vieill. C. tabuensis, Steph.

Friendly, Navigators', and Feejee Islands.

e. Acanthogenys, Gould, 1837.

1418. Meliphaga rufigularis.

B. M. Acanthogenys rufigularis, Gould, B. of Austr.iv. pl. 53. Anthothæra rufogularis, Hartl. Meliphaga rufogularis, G. R. Gr.

New South Wales; S. and W. Australia.

\section{f. Anthochæra, Vig. \&. Horsf. 1826.}

1419. Meliphaga mellivora.

B. $M$.

Certhia mellivora et Merops chrysoptera, Lath. White's Journ. pl. p. 240. C. goruck, Shaw. Philedon goruck, Cuv. Philemon chrysoptera, Vieill. Ois. dor. t. 88. Meliphaga chrysoptera et M. guruck, Steph. Anthochæra mellivora, Vig. \& Horsf. Gould, B. of Austr. iv. pl. 56. Creadion mellivora Vieill. Gould, B. of Austr. ir. pl. 56.

New South Wales; S. Australia and Van Diemen's Land. 
1420. Melipilaga lunulata.

B. M.

Anthochæra lunulata, Gould, B. of Austr. iv. pl. 57.

W. Australia.

1421. Meliphaga paradoxa.

B. M.

Merops carunculata, Lath. White's Voy. pl. p. I44. Corvus paradoxus, Daud. Tr. d'Orn. ii. t. 16. C. carunculatus, Vieill. Meliphaga carunculata, Temm. Anthochæra Lewinii, Vig. \& Horsf. Phil. Voy. Bot. Bay, pl. p. 164. A. carunculata, Gould, B. of Austr. iv. pl. 55. Philedou paradoxus, Cuv.

New South Wales; S. and W. Australia.

1422. Meliphaga inauris.

B. M.

Anthochæra inauris, Gould, B. of Austr. iv. pl. 54. A. carunculata, Vig. \& Horsf. Creadion carunculatus, Vieill. Gal. des Ois. t. 94 .

Van Diemen's Land.

\section{Anthornis.}

a. Anthornis, $G . R . G r .1840$. Anthomyza, $S w, 1837$.

1423. ANTHORnis MELANURA.

B. M.

Certhia melanura, Sparr. Mus. Carls. t. 5. Vieill. Ois. dor. t. 64. C. sannio, Gm. C. olivacea, Forst. Cinnyris melanura. Vieill. Philemon Dumerilii, Less. Voy. de la Coqu. t. 21, f. 2. Anthomyza cæruleocephala, $S w$. Anthornis melanura, $G . R$. Gr. Furnarius? sannio, Steph. Philedon Dumerilii, Cuv. Meliphaga melanura, Hartl.

New Zealand.

1424. Anthornis nelanocephala.

B. M. Anthornis melanocephala, G. R. Gr. Voy. Ereb. \& Terr. Birds, pl. 2.

Chatham's Island, near New Zealand. 
b. Prosthemadera, G. R. Gr. 1840.

1425. Anthornis note zealandie.

B. M.

Merops novæ seelandiæ, Gm. Brown, Illustr.pl. 9. M. concinnata, Lath. Levaill. Ois. de l'Afr. t. 92 . Sturnus crispicollis, Daud. Certhia concinnata, Forst. Meliphaga concinnata, Temm. M. novæ hollandiæ, Steph. Philedon novæ seelandiæ, Cuv. Anthochera novæ zealandiæ, Vig. \&. Horsf. P. circinnatus, Garn. Philemon concinnata, Vieill. Prosthemadera novæ zealandiæ, G. R. Gr.

New Zealand.

\section{Pogonornis.}

Pogonornis, G. R. Gr. 1846.

1426. Pogonornis cincta.

B. M.

Meliphaga cincta, Dubus, Bull. Acad. Sc. Brux. 1839, t. Ptilotis auritus, Lafr. Mag. de Zool. 1840. Ois. t. 11. P. cincta, G. R. Gr. Pogonornis cincta, G. R. Gr.

New Zealand.

\section{Tropidorhynchus.}

a. Tropidorhynchus, Vig.\& Horsf. 1826.

Philedon, p. Cuv. 1817.

Philemon, p. Vieill.

1427. TropidorHYNCHUS CORNICULATUS.

B. M.

Merops corniculatus et M. monachus, Lath. White's Voy. pl. p. 190. Levaill. Ois. de l'Amer. t. 24. Meliphaga corniculata, Temm. M. monacha, Steph. Tropidorhynchus corniculatus, Vig. \& Horsf. Jard. \& Selby, Illustr. of Orn. pl. 133. Gould, B. of Austr. iv. pl. 58. T. diemenensis, Less.? T. monachus, Vig. \&. Horsf. Philedon corniculatus, Cuv. Creadion corniculatus et Philemon monachus, Vieill.

New South Wales.

1428. TROPIDORHYNCHUS ARGENTICEPS.

Tropidorhynchus argenticeps, Gould, B. of Austr. iv. pl. 58.

Voy. au Pole Sud, Ois. t. 18, f. 2.

N. Australia. 
1429. TropidorhyNCHUS GILOLENSIS.

Tropidorhynchus gilolensis, Temm. Pr. B. Consp. p. 390. Gilolo.

1430. TropidorhyNChUS BUCEROIDES.

Philedon buceroïdes, Sw. P. corniculatus, Mus. Par. Less. Tr. d'Orn. t. f. 2. Tropidorhynchus buceroïdes, G. R. Gr. Australia.

1431. TropidorhynchUS CITREOGULARIS. B. M.

Tropidorhynchus citreogularis, Gould, B. of Austr. iv. pl. 60 .

New South Wales.

1432. TropidoRHYNCHUS SORDIDUS.

Tropidorhynchus sordidus, Gould.

N. Australia.

1433. TROPIDORHYNCHUS CHRYSOTIS.

Tropidorhynchus chrysotis, Less. Voy. de la Coqu. t. 21, bis.

Philedon chrysotis, Cur. Myzanthe flaviventer, Less.

New Guinea.

1434. Tropidorhynchus inornatus.

B. M.

Tropidorhynchus inornatus, G. R. Gr. \& Mitch. Gen. of B. pl. 39. T. timoriensis, Müll. \& Schl.? Philedon (vulturin), Voy. au Pole Sud, Ois. t. 18, f. 1 ?

Timor.

1435. TropidorhynChUS NOVæ GUINEF.

Tropidorhynchus novæ guineæ, Mïll. \&- Schl.

New Guinea.

1436. Tropidorhynches subcornutus.

B. M.

Tropidorhynchus subcornutus, Temm. Voy. au Pole Sud, Ois.

t. 16, f. 1. T. subcorniculatus, Homb. \&. Jacq.? 
1437. TROPIDORHYNCHUS BOUROENSIS.

Tropidorhynchus bouroensis, Less. T. buruensis, $\operatorname{Pr}$. B. Philedon bouroensis, Quoy \& Gaim. Voy. de l'Astrol. t. 8, f. 2.

Moluccas (Island of Bouro).

1438. Tropidorhynchus cinerascens.

Tropidorhynchus cinerascens, Miill. Pr. B. Consp. p. 390. Timor.

b. Leptornis, Homb. \& Jacq. 1845.

1439. TropidorhINCHUS SYLVARUM.

Léptornis —? Voy. au Pole Sud, Ois. t. 17, f. 1.

c. Entomyzon, 1825, et Entomyza, 1837, $S w$.

1440. TropidorhynchUS Cyanotis.

B. M.

Gracula cyanotis, Turdus cyaneus et Merops cyanops, Lath. Lew. B. of N. Holl. pl. 4. Meliphaga cyanops, Lew. B. of $N$. Holl. pl. 4. M. graculina, Steph. Entomyzon et Entomyza cyanotis, Sw. Gould, B. of Austr. iv. pl, 68. Philemon cyanops et P. cyanotis, Vieill. Tropidorhynchus cyanotis, Vig. $₫$ Horsf. Certhia graculina, Shaw. Vieill. Ois. dor. t. 87. Gymnops cyanotis et Philedon graculina, Cur.

New South Wales.

1441. Tropidorhynchus albipennis.

B. $\mathbf{M}$ Entomyza albipennis, Gould, B. of Austr. iv. pl. 69. Tropidorhynchus albipennis, $G . R$. $G r$.

N. Australia.

1442. Troptdorhynchus? ANg Ustipluma.

Entomyza? angustipluma, Peale, U. S. Expl. Exped. viii. 147, pl. 40, f. 2 .

Island of Hawaii. 
1443. TropidorhynChUS SAMOENSIS.

Merops samoensis, Hombr. \& Jacq. Tropidorhynchus samoensis, G. R. Gr. Entomyza? olivacea? Peale, U. S. Expl. Exped. viii. p. 145, pl. 40, f. 1.

Navigator's Islands.

1444. ? TROPIDORHYNCHUS MOLUCCENSIS.

Merops moluccensis, Gm. Philedon moluccensis, Cuv. Philemon cinereus, Vieill. Meliphaga moluccensis, Temm. Tropidorhynchus? moluccensis, G.R. Gr.

Molucca.

1445. ?? TROPIDORHYNCHUS? VIRIDIS.

Philemon viridis, Vieill.

Australia.

1446. ?? TropidoRhYNCHUS? N出VIUS.

Philemon nævius, Vieill.

Australia.

5. Phyllornis.

Phyllornis, Boie, 182-?

Chloropsis, Jard. 182-?

1447. Phyllornis Hardwickit.

B. M.

Chloropsis Hardwickii, Jard. \& Selby. C. curvirostris, Sw. C. chrysogaster, McClell. C. auriventris, Deless. Mag. de Zool. 1840. Ois. t. 17. C. cyanopterus, Hodgs. Phyllomis Hardwickii, Hodgs.

India (Nepal).

1448. Phyllornis aurifrons.

B. M.

Phyllornis aurifrons, Temm. Pl. col. 484, f. 1. Chloropsis malabaricus, Jard. \&. Selby, Illustr. of Orn. pl. 5.

India (Assam, Arakan, and Sumatra). 
1449. Phyllornis malabaricos.

B. $M$.

Turdus malabaricus, Gm. Chloropsis aurifrons, Jerd. C. casmarhynchus, Tick. C. malaccensis, Gr. Phyllornis malabaricus, Boie. P. icterucephalus, Less.? P. casmarhynchus, G. R. Gr.

Ceylon.

1450. Phyllornis Jerdoni.

B. M.

Chloropsis gampsorhyuchus, Tick. C. easmarhynchus, $G r$. C. cochinsinensis, Jerd. Ill. Ind. Zool. pl. 43. Phyllornis Jerdoni, $B l$.

India .

1451. Phyllornis cochinchinensis.

B. M. Turdus cochinchinensis, Gm. Pl. enl. 643, f. 3. T. malabaricus (Lath.), Bl. Certhia cosinsinica, Shaw. Vieill. Ois. dor. t. 77, 78. Meliphaga javensis, Horsf. Brachypus? cochinchinensis, Steph. Chloropsis malabaricus, Eyton. C. moluccensis, Gr. Philedon cosinsinica, Cuv. Philemon nigricollis, Vieill. Phyllornis cochinchinensis, Boie. P. moluccensis. G. R. Gr.

India; Java, Sumatra and Borneo.

1452. Phyllornis Sonveratii.

B. $\mathrm{M}$.

Chloropsis Sonneratii et C. gampsorhynchus, Jard. \& Selby, Illustr. of Orn. pl. 100 et 7. C. zosterops, Vig. Turdus viridis, Horsf. Phyllornis Mülleri, Temm. P. Sonneratii, G. R. Gr.

Java, Sumatra and Borneo.

1453. Phylloris media.

Phyllornis media, Müll. Pr. B. Consp. p. 396.

Sumatra.

1454. Phyllornis icterocephala.

Phyllornis icterocephala v. malabaricus, Temm. Pl, cul.512, f. 2 .

Sumatra. 
1455. Phyllornis cyanopogon.

B. M.

Phyllornis cyanopogon, Temm. $\mathrm{Pl}$. col. 512, f. $1 . \quad$ P. mysticalis, G. R. Gr. Chloropsis mysticalis, $S w$.

Sumatra.

1456. Phyllornis venusta.

Phyllornis venusta, Temm. Pr. B. Consp. p. 396.

Sumatra.

\section{iii. Melithreptinæ.}

Melithreptinæ, G. R. Gr. $184 \mathrm{l}-46$; Cab. 1847; Pr. B. 1849.

Manorhininæ, G. R. Gr. 1840.

Meliphaginæ, p. et Timalinæ, p. Pr. B. 1850.

Sturninæ, p. et Turdinæ, p. Pr. B. 1831.

Malurinæ, p. Cab. 1847.

\section{Manorhina.}

Manorhina, Vieill. 1816.

Myzantha, Vig. \&. Horsf. 1826.

Philanthus, Less. 1831.

1457. Manorhina melanophrys.

B. M.

Turdus melanophrys, Lath. Manorhina viridis, Vieill. M. melanophrys, G.R.Gr. Gould, B. of Austr. iv. pl. 80. Myzantha flavirostris, Vig. \& Horsf. Jard. \& Selby, Illustr. of Orn. pl. 78.

New South Wales.

1458. Manorhina garrula.

B. M.

Merops garrulus et Gracula melanocephala, Lath. Philemon garrulus et P. melanocephalus, Vieill. Myzantha garrula, Vig. \&. Horsf. Meliphaga garrula, Steph. "Manorhina garrula, G. R. Gr. Gould, B. of Austr. iv. pl. 76.

New South Wales; S. Australia and Van Diemen's Land.

1459. Manorhina obscura.

Myzantha obscura, Gould, B. of Austr. iv. pl. 77. Manorhina obscura, G.R. Gr.

W. Australia. 
1460. Manorhina flavigula.

Myzantha flavigula, Gould, B. of Austr. pl. 77. Manorhina flavigula, G. R. Gr.

New South Wales.

1461. Manorhina Lutea. B. M.

Myzautha lutea, Gould, B. of Austr. iv. pl. 78. Manorhina lutea, G. R. Gr.

N. Australia.

1462. ? Manorhina citreola.

Myzantha citreola, Gould. Manorhina citreola, G.R. Gr.

Australia.

\section{Psophodes.}

Psophodes, Vig. \& Horsf. 1826.

Timalia, p. Sw.

1463. Psophodes crepitans.

B. M. Muscicapa crepitans et Corvus olivaceus, Lath. Psophodes crepitans, Vig. \&. Horsf. Jard. \& Selby, Illustr. of Orn. pl. 60. Gould, B. of Austr. iii. pl. 15. Meliphaga crepitans, Steph. Pica olivacea, Vieill. P. gularis, Wagl. Dasyornis Abeillei, Less. Timalia crepitans, $\$ w$.

New South Wales.

1464. Psophodes nigrogularis.

B. M.

Psophodes nigrogularis, Gould, B. of Austr. iii. pl. 16.

W. Australia.

\section{Sphenostoma.}

Sphenostoma, Gould, 1837.

1465. Sphenostoma CRistatum.

Sphenostoma cristatum, Gould, B. of Austr. pl.

Australia. 


\section{Melithreptus.}

a. Melithreptus, Vieill. 1816.

Hæmatops, Gould, 1836.

Eidopsarus et Gymnophrys, $S w .1837$.

1466. Melithreptus lunatus.

B. M.

Certhia lunata, Shaw. Vieill. Ois. dor. t. 61. Meliphaga lunata, Steph. M. lunulata et M. atricapilla, Temm. Pl. col. 335, f. 1. M. torquata, Sw. Zool. Illustr. pl. 116. Philedon luvatus, Cuv. Melithreptus lunulatus, Vieill. Gould, Jard. \&. Selby, Ill. of Orn. pl. 134, f.2. Hæmatops lunulatus, Gould, B. of Austr. iv. pl. 72. Gymnophrys torquatus, Sw.

New South Wales and S.Australia.

1467. Melithreptus atricapillus.

Certhia atricapilla, Lath. Meliphaga atricapilla, Temm. Melithreptus atricapillus, Vieill. Philedon atricapillus, Cuv.

Australia.

1468. Melithreptus Chloropsis.

Melithreptus chloropsis, Gould, B. of Austr. iv. pl. 73.

W. Australia.

1469. Melithreptus albigularis.

Melithreptus albigularis, Gou!d, B. of Austr. iv. pl. 74.

New South Wales and N. Australia.

1470. Meltitheptus melanocephalus.

B. M. Melithreptus melanocephalus, Gould, B. of Austr. iv. pl. 75. Meliphaga atricapilla, Jard. \&. Selby, Illustr. of Orn. pl. 134, f. 1. Eidoplsarus affinis, Less.

Van Diemen's Land.

1471. Melithreptus virescens: B. M.

Hæmatops validirustris, Guuld, B. of Austr. iv. pl. 70. Sturnus virescens, Wayl. Eidopsarus hicinctus, Sw. Melithreptus virescens, G. R. Gr. MI. validirostris, Gould. Voy. au Pole Sud, Ois. t. 17, f. 1.

Van Diemen's Land. 
1472. Melithreptus gularis.

B. M.

Hæmatops gularis, Gould. Melithreptus gularis, G. R. Gr. Gould, B. of Austr. iv. pl. 71 .

New South Wales and S. Australia.

1473. ? Melithreptus pipilans.

Certhia pipilans, Lath. Melithreptus pipilans, Vieill. M. atricapillus, p.? G.R. Gr. Meliphaga pipilans, Steph.

Australia.

1474. ?Melithreptus inornatus.

Sylvia inornata, Lath. Motacilla inornata, Vieill. Melithreptus? inornatus, G. R. Gr.

Australia.

1475. MELITHREPTUS INDISTINCTUS.

Meliphaga indistincta, Vig. \& Horsf. Melithreptus indistinctus, G. R. Gr.

Australia.

1476. ? MeLithreptus BREVirostris.

Meliphaga brevirostris, Vig. \&. Horsf. Melithreptus brevirostris, G. R. Gr.

Australia.

1477. ?? MelithreptUS ALBICA PILLUS.

Melithreptus albicapillus et M. albicollis? Vieill.

Australia.

b. Plectroramphus, G. R. Gr. 1840 .

Plectrorbyncha, Gould, 1837.

1478. Melithreptus lanceolatus.

B. M.

Plectrorhyncha lanceolata, Gould, B. of Austr. iv. pl. 47. Plectroramphus lanceolatus et Melithreptus lanceolatus, $G$. R. $G r$.

New South Wales. 


\section{CERTHIAD艮.}

Certhiadæ, Vigors, 1825 ; Sw. 1831-37; Pr. B. 1838-49-50; G.R. Gr. $1840-41-46 ; C a b .1847 ; B l .1849$.

Anabatidx, Cab. $1847 ;$ Pr. B. 1849-50.

Trochilidæ, p. 1831 et Maluridæ, p. $1850, \operatorname{Pr} . B$.

Sylviadæ, p. Vigors, 1825; Sw. 1827; D'Orb. \& Lafr. 1838.

Dendrocolaptidæ, Selys, 1842 ; Pr. B. 1850.

Tichodromidæ et Megapodiidæ, p. Selys, 1842.

Cracidæ, p. Vigors, 1825.

Menuridæ, p. Pr. B. $1849-50$.

Timalidæ, p. Liotrichidæ, p. et Eriodoridæ, p. Cab. 1847.

Brachyuridæ, p. $B l .1849$.

Uppucerthidæ, $D^{\prime} O r b$. \& Lafr. 1838.

Timalides, p. Anabatides, Certhiades et Menurides, Sundev. 1835.

\section{i. Furnarinne.}

Furnariinæ, G. R. Gr. 1840-41-46, Cab. 1847 ; Pr. B. $1849-$ 50.

Certhianæ, p. Pr. B. 1831 ; Sw. 1837.

Anabatina, p.; Sittinæ, p. et Troglodytinæ, p. Sw. 1837.

Tichodromina? p. Sw. 1827.

Upupinæ, p. Pr. B. 1831 .

\section{Furnarius.}

Furnarius, Vieill. 1816.

Opetiorhynchus, Temm. 1820.

Figulus, Spix, 1824.

1479. Furndrius rufus.

B. M.

Merops rufus, Gm. Turdus badius, Licht. Furmarius rufus,

Vieill. F. ruficaudus, Harll. Opetiorhynchus ruficaudus, $P r$.

Max. Figulus albogularis, Spix, Av. Bras. t. 78, f. .

Nectarinia rufa, Cuv.

S. America (Brazil). 
1480. Furvarius figulus.

B. M.

Turdus figulus, $I l l$. Opetiorhynchus rufus, Pr. Max. Furnarius figulus, G. $R$. $G r$. F. melanotis, $S w$. T. superciliaris, Less.? F. rufus, Hartl.?

S. America (Brazil).

1481. Furnarius rectirostris.

Opetiorhynchus rectirostris, $\operatorname{Pr}$. Max. Furnarius rectirostris, G. R. Gr.

S. America (Brazil).

1482. Furnarius roseus.

Furnarius roseus, Less. Illustr. de Zool. t. 5.

B. M.

S. America.

1483. Furnarius griseus.

Furnarius griseus, $S w$. Campylorbynchus griseus, $\mathrm{Cab}$.

B. M.

S. America (Guiana).

1484. Furnarius levcopus.

B. M.

Furnarius leucopus, $S w$.

S. America (Guiana).

1485. Furnarius longipennis.

Furnarius longipennis, $S w$.

S. America (Peru).

1486. Furnarius fasciatus.

Furnarius fasciatus, $S w$.

S. America (Peru). 


\section{Cinclodes.}

a. Cinclodes, G. R. Gr. 1840.

Cillurus, Cab. 1844.

1487. Cinclodes patagonicus.

B. M.

Motacilla patagonica, $G m$. M. gracula, Forst. Sylvia patagonica, Lath. Dixon's Voy. pl. p. 359. Furnarius Lessonii, Dum. F. chilensis, Less. Tr. d'Orn. t. 75, f. 1. F. patagonicus, Hartl. Opetiorhynchus rupestris, Kittl. Mem. de l'Acad. St. Petersb. 1831, t. 1. O. patagonicus, G. R. Gr. Uppucerthia rupestris, $D^{\prime} O r b$. \& Lafr. Cinclodes patagonicus, $G$. R. Gr.

S. America (Patagonia, Chili, Bolivia).

1488. Cinclones vulgaris.

B. M.

Uppucerthia vulgaris, D'Orb. \& Lafr. Voy. dans $\mathrm{l}$ Amer. Mer. Ois. t.57, f. 1. Opetiorhynchus vulgaris et Cinclodes vulgaris, G. R. Gr.

S. America (La Plata, Patagonia, Bolivia).

1489. Cinclodes antarcticus.

B. M.

Certhia antarctica et Furnarius fuliginosus, Less. Opetiorhynchus antarcticus, G. R. Gr. Cinclodes antarcticus, G. R. Gr. C. fuliginosus, Less.

S. America.

1490. Cinclodes nigrofumosus.

B. M.

Uppucerthia nigrofumosa, D'Orb. \& Lafr. Voy. dans l'Amer. $M e r$. Ois. t. 57, f. 2. Opetiorhynchus nigrofumosus, $G$. $R$. Gr. O. lanceolatus, Gould, Voy. of Beagle, Zool. pl. 20. Cinclodes nigrofumosus, $G . \boldsymbol{R}$. $\boldsymbol{G} r$. C. inornatus, Less.

S. America (Bolivia, Peru).

1491. Cinclodes montanus.

B. M.

Uppucerthia montana, D'Orb. \&. Lafr. Voy. dans $l$ Amer. Mer. Ois. t. 56, f. 1. Cinclodes montanus, G. $R$. Gr.

S. America (Peru). 
1492. Cinclodes andecola.

B. $M$.

Uppucerthia andæcola, D'Orb. \& Lafr. Voy. dans l'Amer. Mer.

Ois. t. 56, f. 2. Cinclodes andæcola, G. R. Gr.

S. America (Bolivia).

1493. Cinclodes paliatus.

Cinclodes palliatus, Tschudi, Fauna Per. t. 16, f. 2.

S. America (Peru).

b. Upucerthia, I. Geoffr. 1832.

1494. Cinclodes dumetorius.

B. $M$.

Upucerthia dumetoria, I. Geoffi. \&. D'Orb. Uppucerthia dumetorum, D'Orb. \& Lafr. Voy. of Beagle, Birds, pl. 19. Cinclodes dumetorius, G. R. Gr.

S. America (Chili).

c. Ochetorhynchus, Meyen, 1834.

1495. Cinclodes ruficaudus.

Ochetorhynchus ruficaudus, Meyen, Nov. Act Acad. Nat. Cur. xvi. Suppl. t. 11. Uppucerthia rupestris, p. D'Orb. \&.

Lafr. Cinclodes ruficaudus, G. R. Gr.

S. America (Chili, Bolivia).

\section{Lochmias.}

Lochmias, Sw. 1827.

Picerthia, I. Geoffr. 1832.

1496. Lochmias St. Hilarii.

B. M.

Furnarius St. Hilarii, Less. Myiothera nematura, Licht. Lochmias squamatula, $S w$. B. of Braz. pl. 38. L. St. Hilarii, G.R. Gr. L. nematura, Cab. Myrmothera nematura, Menétr.

S. America (Brazil). 


\section{Enicornis.}

Enicornis, G. R. Gr. 1840.

Eremobius, Gould, 1839.

Henicornis, $\operatorname{Pr} . B .1850$.

1497. Enicornis Phenicura.

Eremobius phænicura, Gould, Voy. of Beagle, Birds, pl. 21.

Enicornis phænicura, $G, R$. $G r$.

S. America (Patagonia).

1498. Enicornis metanura.

B. M.

Enicornis melanura, G. R. Gr. \& Mitch. Gen. of B. 1, pl.

S. America (Chili).

\section{Limnornis.}

Limnornis, Gould, 1839.

Cinnicerthia, Less.

1499. LIMNORNIS CURVIROSTRIS.

Limnornis curvirostris, Gould, Voy. of Beagle, Birds, pl. 25.

S. America (La Plata).

1500. ? LIMNORNis RECTIROSTRIS.

B. M.

Limnornis rectirostris, Gould, Voy. of Beayle, Birds, pl. 24.

S. America (La Plata).

1501. LIMNORNIS UNIROFUS.

Limnornis unirufus, Lafr. Cinnicerthia cinnamomea, Less.

S. America (New Granada).

1502. LimNorxis Canifrons.

Limnornis canifrons, Lafr.

S. America (New Granada). 


\section{Geositta.}

a. Geositta, $S w .1837$.

1503. Geositta cunicularia.

B. $M$.

Alauda cunicularia, Vieill. A fissirostris, Kittl. Mern. de l Acad. St. Petersb. 1835, t. 3. Geositta anthoïdes, Sw. G. cunicularia et Furnarius cunicularius, G.R.Gr. Certhilauda cunicularia, D'Orb. \& Lafr. Voy. dans l'Amer. Mer. Ois. t. 43, f. 1.

S. America (Paraguay, La Plata, Bolivia, Chili).

1504. Geositta peruviana.

Geositta peruviana, Lafr.

S. America (Peru).

1505. Geositra tenuirostris.

B. II.

Certhilauda tenuirostris, D'Orb. \& Lafr. Voy. dans TAmer. Mer. Ois. t. 43, f. 2. Gensitta tenuirostris, G.R. Gr.

S. America (Bolivia).

1506. Geositta maritima.

Certhilauda maritima, D'Orb. \& Lafr. Voy. dons I Amer. Mer Ois. t. 44, f. 1. Geositta maritima, G. R. Gr.

S. America (Bulivia).

b. Geobates, Sw. 1837 .

1507. Geositta Brevicauda.

B. II.

Geobates brevicauda, $S w$. Geositta brevicauda, G. R. Gr.

S. America (Brazil).

\section{Cinclocerthis.}

Cinclocerthia, G. R. Gr. 1840.

Stenorhynchus, Gould, 1835.

1508. Cinclocerthia ruficauda.

B. MI

Stenorhynchus ruficaudus, Gould. Cinclocerthia ruficauda, Gould.

W. Indies (St. Nevis). 
ii. Synallaxine.

Synallaxinæ, G. R. Gr. $1846 ;$ Pr. B. $1849 ; B l .1849$.

Certhianæ, $S w .1827$.

Sylviana, p. Vigors, 1825.

Sylvinæ, p. Sittinæ, p. et Certhinæ, p. Pr. B. 1831.

Oxyrhynchinæ, Sw. 1831 .

Dacnidinx, p. Cab. 1847.

Anabatinæ, Sw. 1837; G. R. Gr. $1840-41$; Cab. 1847 ; Pr. B. $1849-50$.

\section{Synallaxis.}

a. Synallaxis, Vieill. 1818.

Parulus, Spix, 1824.

1509. Synaliaxis RUficapilla.

B. M.

Synallaxis ruficapilla, Vieill. Gal. des Ois. t. 174. S. albescens, Temm. Pl. col. 227, f. 2 ? S. cinereus, Pr. Max. S. pusillus, Nordm.? Sphenura ruficeps, Licht. Parulus ruficeps, Spix, Av. Bras. t. 86, f. 2.

S. America (Brazil, Guiana, Bolivia).

1510. ? Synallaxis Azaræ.

B. M.

Synallaxis Azaræ, D'Orb.

S. America (Paraguay).

1511. Synalulaxis humicola.

B. M.

Synallaxis humicola, Kittl. Mem. l Acad. des Sci. Petersb. 1830 , t. 6 .

S. America (Chili).

1512. Synallaxis Boliviensis.

B. M.

Synallaxis humicola, D'Orb. \& Lafr. Voy. dans l'Amer. Mer.

Ois. t. 17, f. 2.

S. America (Boliria). 
1513. Synallaxis eggithaloides.

Synallaxis ægithaloides, Kittl. Mem. l'Acad. des Sci. Petersb. 1830, t. 7. S. Thelotii, Less.

S. America (Chili, Patagonia, Bulivia).

1514. Synallaxis LevCocephata.

Synallaxis leucocephala, $D^{\prime} O r b . \& L a f r$.

S. America (Patagonia).

1515. Synallaxis Fuliginiceps.

Synallaxis fuliginiceps, $D^{\prime} O r b$. \& Lafr. Voy. dans $l$ Amer. Mer. Ois. t. 17, f. 1 .

S. America (Bolivia).

1516. Synallaxis Candei. Synallaxis Caudei, D'Orb. \& Lafo.

S. America (Carthagena).

1517. Synallaxis Rutilans.

Synallaxis rutilans, Temm. $P l . c o l .227$, f. 1.

S. America (Brazil).

1518. Syxat.laxis cinerascens.

Synallaxis cinerascens, Temm. Pl. col. $22 \pi$, f. 3.

S. America (Brazil).

1519. SyNallaxis Setaria.

Synallaxis setaria, Temm. Pl. col. 311, f. 2.

S. America (Brazil).

1520. Syyalaxis Rufigularis.

B. I.

Synallax is rufogularis, Gould, Voy. of Beagle, Birds, pl. 23.

S. America (Patagonia, Chili).

1521. SyNallaxis BrorNea.

Synallaxis brunnea, Gould.

S. America (Patagonia). 
1522. Symallaxis sordida. Synallaxis sordida, Less. Beagle, Birds, pl. 24.

B. M. S. flavogularis, Gould, Voy. of S. America (Patagonia).

1523. Synallaxis Pallida.

Synallaxis pallida, Pr. Max.

S. Amer:ca (Brazil).

1524. Synallaxis melanops.

B. M. Synallaxis melanops, Vieill. S. dorsomaculata, D'Orb. \&.Lafr. Voy. dans l'Amer. Mer. Ois. t. 14, f. 2.

S. America (Paraguay, La Plata).

1525. Syrallaxis TORQUata.

B. M. Synallaxis torquata, $\mathrm{Pr}$. Max. S. bitorquata, Lafr. \& D'Orb. Voy. dans l'Amer. Mer. Ois. t. 15, f. 2.

S. America (Bolivia).

1526. Synallaxis Maximitiani.

B. M.

Synallaxis torquata, D'Orb. \& Lafr. Voy. dans IAmer. Mer.

Ois. t. 15, f. 1. S. Maximiliani, D'Orb.

S. America (Paraguay, Bolivia).

1527. Synallaxis patagonica.

Synallaxis patagonica, $D^{\prime} O r b$.

S. America (Patagonia).

1528. Srnallaxis albiceps.

B. MI.

Synallaxis albiceps, D'Orb. \& Lafr. Voy.dans l'Amer. Mer. Ois.

t. $16, \mathrm{f} .2$.

S. America (Bolivia).

1529. SYNALLAXIS STRIATICEPS.

Synallaxis striaticeps, D'Orb. \& Lafr. Voy. dans l'Amer. Mer. Oie. t. $16, \mathrm{f} .1$.

S. America (Bolivia, La Plata). 
1530. Synallaxis troglodytoides.

Synallaxis troglody toides, $D^{\prime} O r b$.

S. America (Patagonia).

1531. Synaliaxis Ruficauda.

Synallaxis ruficauda et Sylvia russeola, Vieill. S. caudacutus, Pr. Max. Sphenura mentalis, Licht. Parulus ruficeps, Spix, Av. Bras. t. 86, f. 1. Opetiorbynchus inundatus, Temm.

S. America (Brazil).

1532. SynalLaxis MaLUROIDES.

Synallaxis maluroides, D'Orb. \& Lafr. Voy. dans l'Amer. Mer. Ois. t. 14, f. 3, 4 .

S. America (La Plata).

1533. Synatlaxis anthoides.

Synallaxis anthoïdes, King.

S. America (Patagonia).

1534. Synallaxis Phrygaxophila.

B. M.

Synallaxis phryganophila, Vieill. S. tecellata, Temm. Pl. col. 3 il 1, f. 1.

S. America (Brazil, Paraguay, La Plata).

1535. SrNallaxis CinNaMomea.

Certhia cinnamomea, Gm. Vieill. Ois. dor. t. 62. Gal.des Ois. t. 173. Synallaxis cinnamomea, $S w$. S. ruficauda, Spix, Av. Bras, t. 85, f. 2. Sphenura cinnamomea, Licht.

S. America (Guiana).

1536. Synaltaxis Lafresnayi.

Synallaxis cinnamomeus, Lafr.

S. America (Columbia).

1537. Synalitaxis striaticollis.

Synallaxis striaticollis, Lafr.

S. America (Columbia). 
1538. Syxallaxis unirufa.

Synallaxis unirufus, Lafr.

S. America (Columbia).

1539. Synallaxis Fuliginosa.

B. M. Synallaxis fuliginosus, Laf $r$.

S. America (Columbia).

1540. Synatuaxis BRACHyURUs.

Symallaxis brachyurus, Lafr.

S. America (Columbia).

1541. Synatlaxis golaris.

Synallaxis gularis, Lafr .

S. America (Columbia).

1542. Synaliaxis terrestris.

B. MI.

Synallaxis terrestris, Jard.

Island of Tobago.

1543. Synallaxis Flammulatus.

Synallaxis flammulatus, Jard. Contr. Ornith. $1850, \mathrm{pl}$.

S. America (Quito).

1544. Synallaxis

Picolaptes

f. 1. Synallaxis ou Oxyurus, Lafr.

S. America.

1545. Syxallaxis griseicapilla.

Dendrocopus griseicapillus, Vieill. Dendrocolaptes griseicapillus, G. R. Gr. Synallaxis ou Anabates, Lafr.

S. America (Paraguay). 
b. Oxyurus, $S w .1827$.

1546. Synallaxis Spinicauda.

B. M.

Motacilla spinicauda, Gm. M. seticauda, Forst. Sylvia spinicauda, Lath. Certhia spinicauda, Temm. Oxyurus australis et O. ornatus, $S w$. O. patagonicus, Less. O. Supinieri, Gould. O. spinicaudus, G. R. Gr. Synallaxis Supinieri, Less. Voy. de la Coqu. t. 29, f. I. S. spinicauda, G. R. Gr.

S. America (Patagonia, Chili, Island of Chiloë).

c. Sylviorthorhynchus, O. Des Murs, 1847. Schizura, Cab. 1848.

1547. Synallaxis Des Merit.

Sylviorthorhynchus maluroides, O. Des Murs, Iconogr. Ornith.

t. 45. S. Des Murii, Gay. Schizura maluroides, Cab.

S. America (Chili).

\section{Anumbius.}

Anumbius, D'Orb. \& Lafr. 1838.

Sphenopyga, Cab, 1847.

Synallaxis, $S w .1837$.

1548. Anumbius acuticaudatus.

B. $M$.

Anthus acuticaudatus, Less. Anumbius anthoides, $D^{\prime} O r b$. \&

Lafr. A. acuticaudatus, G.R. Gr. Synallaxis major, Gould, Voy. of Beagle, Birds, pl. 22. Furnarius annumbi, Vieill.

S. America (Paraguay, La Plata).

1549. Anumbius ruber.

B. M.

Anumbius ruber, D'Orb. \& Lafr. Furnarius ruber, Vieill.

S. America (La Plata, Bolivia).

1550. Anumbius striaticolis.

Anumbius striaticollis, D'Orb. \& Lafr.

S. America (La Plata). 
155l. ANumbius striaticeps.

Anumbius striaticeps, D'Orb. \& Lafr.

S. America (Bolivia).

1552. Anembius RUfifrons.

B. M

Anabates rufifrons, Spix, Av. Bras. t. 85, f. 1. Sphenura frontalis, Licht. Malurus garrulus, Sw. Zool. Illustr. pl. 138. Anumbius rufifrons, $D^{\prime} O r b . \&$ Lafr. Synallaxis rufifrons, G. R. Gr.

S. America (Brazil, Bolivia).

\section{Diglossa.}

Diglossa, Wagl. 1832.

Serrirostrum (1838) et Uncirostrum (1839), D'Orb. \& Lafr.

Anchilorhinus et Agrilorhinus, $\operatorname{Pr} . B .1838$.

Campylups, Licht. 1837.

1553. Diglossa baritula.

B. M.

Diglossa baritula, Wagl. G.R.Gr. \&-Mitch. Gen. of B. i. pl. 42. Uncirostrum Brelayi, Lafr. Agrilorhinus vel Anchilorhinus sittaceus, $\mathrm{Pr}$. B. Campylops hamulus, Licht. Kuster, Voeg. Laniada, t. 1.

S. America (Mexico).

1554. Diglossa D'Orbignyit.

Uncirostrum D’Orbignyii, Boiss. Diglossa baritula, p. $G$. R. Gr. D. D'Orbignyi, Hartl.

S. America.

1555. Diglossa sittoides.

B. M.

Serrirostrum sittoïdes, D'Orb. \&. Lafr. Voy. dans l'Amer. Mer. Ois. t. 58, f. 2. Agrilorhinus olivaceus, Fras. Diglossa sittoïdes, Hartl.

S. America (Bolivia).

1556. Diglossa similis.

Diglossa similis, Lafr.

S. America (New Granada). 
1557. Diglossa mystacalis. Diglossa mystacalis, Lafr. Gen. of B. i. pl. 42 , f. 1 .

S. America (Bolivia).

155̃8. Diglossa brunneiventris.

B. M.

Diglossa brunneiventris, O. Des Murs. Iconogr. Ornith. t. 43.

S. America (Peru).

1559. Diglossa aterrima.

B. M.

Diglussa aterrima, Lafr.

S. America (New Granada).

1560. Diglossa Lafresnayit.

B. M.

Uncirostrum Lafresnayii, Boiss. Diglossa Lafresnayii, Hartl. Agrilorhinus Bonapartei, Fras. Diglossa Bonapartei, $\operatorname{Pr}$. B.

S. America (New Granada).

1561. Diglossa humeralis.

B.M. Agrilorhinıs humeralis, Fras. Diglossa Lafresnayii, p. G. R. Gr. D. humeralis, Hartl.

S. America (New Granarla).

1562. Diglossa CYANEA.

B. M.

Uncirostrum cyaneum, Lafr. Agrilorhinus personatus, Fras. Diglossa cyanea, G. R. Gr. D. melanopis, Tschuli. D. personata, Hartl.

S. America (New Granada, Peru).

1563. Diglossa albilatera.

B. M. Diglossa albilatera, Lafr.

S. America (Columbia).

1564. Diglossa major.

Diglossa major, $\mathrm{Cab}$.

S. America (Guiana). 
1565. Diglossa carbonaria.

B. M.

Serrirostrum carbonarium, D'Orb. \& Lafr. Voy. dans l'Amer. Mer. Ois. t. 58, f. 1. Diglossa carbonaria, Hartl.

S. America (Bolivia).

\section{Anabates.}

a. Anabates, Temm. 1820 .

Anabacerthia, Lafr. 18 ??

1566. Anabates guianensis.

Motacilla guianensis, $G m$. Pl. enl. 686, f. 2. Sylvia guianensis, Lath. Anabates ruficaudus, Temm. A. guianensis, Steph.

S. America (Guiana).

1567. ANaBATES AMAURoTIs.

Anabates amaurotis, Temm. Pl.col. 238, f. 2. Xenops nigricapillus, Less.

S. America (Brazil).

1568. Anabates subulatus.

Sphenura subulatus, Spix, Av. Bras. t. 83, f. 1. Anabates subulatus, G. R. $G r$.

S. America (Brazil).

1569. Anabates cristatus.

B. M.

Anabates cristatus, Spix, Av. Bras. t. 84.

S. America (Brazil, La Plata).

1570. ANabates unirufus.

Anabates unirufus, D'Orb. \& Lafr Voy. dans l'Amer. Mer. Ois. t. 55 , f. 1 .

S. America (Bolivia).

1571. Anabates gutturalis.

Anahates gutturalis, D'Orb. \&. Lafr. Voy. dans l'Amer. Mer. Ois. t. 55, f. 3 .

S. America (Patagonia). 
1572. Anabates subcristatus.

Anabates subcristatus, Sw. B. of Bras. pl. 81. A. lophotes, S. America.

1573. Anabates leucopthalmus. B. M. Anabates leucopthalmus, Pr. Max. Sphenura sulphurascens, Licht. Xenops gularis, Vlenc. Philydor albogularis, Spix, Av. Bras. t. 74. Anabates albogularis, Cuv.

S. America (Brazil).

1574. ANaBATES ERYTHROPTHALMUS.

Anabates erythropthalmus, $\boldsymbol{P r}$. Max. O. Des Murs, Iconogr. Ornith.t. 44.

S. America (Brazil).

1575. Anabates ferruginolentus.

Anabates ferruginolentus, $\boldsymbol{P r}$. Max.

S. America (Brazil).

1576. Anabates ARADOIDES.

Anabates aradoides, Lafr. Mag. de Zool. 1832. Ois. t. 8.

S. America.

\section{Anabates RUficaudatus.}

Anabates ruficaudatus, D'Orb. \& Lafr.

S. America (Bolivia).

1578. Anabates certhioides.

Anabates certhioides, D'Orb. \& Lafr.

S. America (La Plata).

1579. Anabates montanus.

Anabates montanus, Tschudi, Fauna Per. t. 20, f. 2.

S. America (Peru).

1580. Anabates OCHRolemus.

Anabates ochrolæmus, Tschudi, Fauna Per. t. 20, f. 2.

S. America (Peru). 
1581. Anabates melanorhynchus.

Anabates melanorhynchus, Tschudi, Fauna Per. t. 21, f. 1.

S. America (Peru).

1582. Anabates striatus.

B. M.

Anabates (Sphenura) striatus, Spix, Av. Bras. t. 83, f. 2. (striolatus). A. ruficollaris, G.R.Gr. A. striolatus, Hartl. Xenops ruficollaris, Less. Cent. de Zool. t. 36. Thripophaga striolata, p. $\operatorname{Pr} . B$.

S. America (Brazil).

1583. Anabates striaticollis.

Anabates striaticollis, Lafr. Anabacerthia,- Lafr.

S. America.

1584. Anabates nigropectus.

Anabates nigropectus, Lafir. Rev. et Mag. de Zool. 1850, p. t. 1, f. 3 .

S. America.

1585. Anabates pyrrhodes. Anabates pyrrhodes, $\mathrm{Cab}$.

S. America (Guiana).

1586. Axabates squamiger. B. M.

Sittasomus perlatus, Less. Anabates squamiger, D'Orb. \& Lafr. Voy. dans l Amer. Mer. Ois. t. 54, f. 2.

S. America (Bolivia).

1587. Anabates gutturatus.

Anabates gutturatus, D'Orb. \& Lafr.

S. America (Bolivia).

1588. Anabates strigllatus.

Thamnophilus strigilatus, Spix, Av. Bras. t. 36, f. 1.

S. America (Brazil). 
1589. Anabates? maculatus.

Dendrocopus maculatus, Vieill. Dendrocolaptes maculatus, G. R. Gr. Dendrocolaptes (Xiphocolaptes) maculatus, Less. Anabates? maculatus, Lafr.

S. America (Paraguay).

1590. ANabates? miniatus.

Dendrocolaptes miniatus, $\mathrm{Ill}$. Dendrocopus rubricaudatus, Vieill. Dendrocolaptes (Xiphocolaptes) rubricaudatus, Less. Picolaptes miniatus, G. R. Gr. Anabates ou Synallaxis? Lafr.

S. America (Paraguay).

1591. Anabates? superciliosus.

Dendrocolaptes superciliosus, Licht. Dendrocopus pyrrbophæus, Vieill. Dendrocolaptes (Xiphocolaptes) pyrrophius, Less. Picolaptes superciliosus, G. R. Gr. Anabates ou Synallaxis? Lafr.

S. America (Paraguay).

b. Philydor, Spix, 1824.

Dendroma, $S w .1837$.

1592. Anabates poliocephalus."

B. M.

Sphenura poliocephala, Licht. Philydor ruficollis, Spix, Av. Bras. t. 75. Xenops rufifrons, Valenc. Anabates poliocephalus, $G . R$. Gr. A. ruficollis, Cuv. Dendroma caniceps, $S w$. D. poliocephalum, Harth. " Dendrocopus rufus, Vieill." Lafr. Dendrocolaptes rufus, G. R. Gr.

S. America (Brazil).

\section{c. Thripophaga, Cab. 1847.}

1593. Axabates macrourus.

Sphenura striolata, Licht. Anabates striolata, Temm. Pl. col. 288, f. 1. A. macrourus, $\operatorname{Pr}$. M. Thripopbaga striolata, $\operatorname{Pr}$. $B$.

S. America (Brazil). 
1594. Anabates Boissonneautil. Anabates Boissonneautii, Lafr. A. auritus, Licht. Tripophaga aurita, $\mathrm{Cab}$.

S. America (New Granada, Peru).

\section{OXYRHAMPHUS.}

Oxyrhamphus, Strickl. 18 ??

Oxyrhynchus, Temm. 1820.

1595. OXYRAMPHUS FLAMMICEPS.

B. M.

Oxyrhynchus flammiceps, Temm. Pl. col. 125. O. cristatus, Sw. Zool. Illustr. pl. 49. O. serratus, Mikan. Delic. Flor. et Fauna Bras. t. Oxyramphus flammiceps, Strickl.

S. America (Brazil).

\section{DENDROCOLAPTIN正.}

Dendrocolaptinæ, G. R. Gr. 1840-41-47; Cab. 1847 ; Pr. B. $1849-50 ; B l .1849$.

Certhianæ, p. Pr. B. $1831 ; S w .1837$.

Certhiana, $S w .1827$.

\section{Dendrocolaptes.}

a. Dendrocolaptes, Herm. 1811.

Dendrocopus, Vieill. 1816.

Orthocolaptes et Xiphocolaptes, Less. 1840.

1596. Dendrocolaptes Certhia.

B. M.

Picus certhia, Bodd. Pl. enl. 621. Gracula cayanensis, Gm.

G. scandens, Lath. Vieill. Ois. dor. t. 79. Levaill. Promer. t.

26. Dendrocolaptes cayanensis, Licht. D. major, Hoffm.

D. picumnus, Licht. $\quad$ D. communis, Less. D. certhia, $G . R$.

Gr. Dendrocopus scandens, Vieill. Dendrocolaptes (Orthocolaptes) cayanensis, Less.

S. America (Guiana, Bolivia, Honduras?) 
1597. ? Dendrocolaptes validus.

Dendrocolaptes validus, Tschudi, Fauna Per. t. 21, f. 2. D. cayanensis, p. Lafr. Dendrocops validus, $\operatorname{Pr}$. B.

S. America (Peru).

1598. ? DendRocolaptes CRassirostris.

Dendrocolaptes crassirostris, Such. D. cayanensis rel D. platyrostris, p. Lafr.

S. America (Brazil).

1599. Dendrocolaptes Major.

B. M.

Dendrocopus major, Vieill. Dendrocolaptes rubiginosus, Lafr. Mag. de Zool. 1833. Ois. t. 16. D. major, G. R. Gr. Dendrocolaptes (Xiphocolaptes) major, Less.

S. America (Paraguay, Bolivia).

1600. ? Dendrocolaptes CYanotis.

Dendrocolaptes cyanotis, Licht. Levaill. Promer. t. 25. D. major, p. vel D. falcirostris, p. Lafr.

S. America.

1601. Dendrocolaptes decumanus.

B. II.

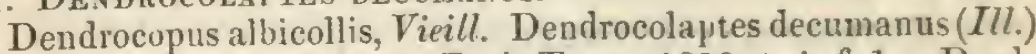
et D. picumnus, p. Licht. Berl. Trans. 1820, t. I, f. 1. D. albicollis, D'Orb. \& Lafr. Dendrocolaptes (Xiphocolaptes) decumanus, Less. Spix, Ax. Bras. t. 87.

S. America (La Plata, Bolivia).

1602. Dendrocolaptes falcirostris.

B. M.

Dendrocolaptes falcirostris, Spix, Av. Bras. t. 88. D. albicollis, p. G. R. Gr. Dendrocolaptes lineatocephalus, $G . R$. Gr. \& Mitch. Gen. of B. i. pl. . Dendrocops lineatocephalus, $\operatorname{Pr}$. B.

S. America (Brazil).

1603. Dendrocolaptes promeropirhyNChris.

B. M. Dendrocolaptes (Xiphocolaptes) promeropirhynchus, Less. $\mathrm{Pi}$ colaptes promeropillynchus, G. R. Gr. D. meropirostris, Lafr.

S. America. 


\section{b. Dendrocops, $S w .1837$.}

Premnocopus, Cab. 1847.

1604. Dendrocolaptes platyrostris.

B. $\mathbf{M}$.

Dendrocolaptes platyrostris, Spix, Av. Bras. t. 89. Dendrocups platyrostris, $S w$.

S. America (Brazil).

1605. ? DENDRocolaptes MELANOCEPS.

Dendrocolaptes (Orthocolaptes) melanoceps, Less. D. platyrostris, p. Lafr. Dendrocops melanoceps, $P r$. B.

S. America.

1606. Dendrocolaptes multistrigatus.

Dendrocolaptes multistrigatus, Eyton.

S. America.

1607. DENDROCOLAPTES FortiRostris.

Dendrocolaptes fortirostris, Such. Dendrocops fortirostris, Pr. B.

S. America (Brazil).

1608. Dendrocolaptes undulatus.

Premnocopus undulatus, $\boldsymbol{C a b}$.

S. America (Guiana).

c. Dendroplex, Sw. 1827.

1609. Dendrocolaptes picus.

B. 11 .

Dendrocolaptes picus, Licht. D. minor, Herm. D. chrysolojhus, Ill. D. rectirostris, $D^{\prime} O r b$. \&. Lafr. Oriolus picus, Gm. Pl. enl. 605. O. picoides, Shaw. Dendrocopus rectirostris, Vieill. Dendrocolaptes (Orthocolaptes) picus, Less. Dendroplex — ? Sw. Levaill. Promer. t. 27.

S. America (Guiana, Brazil, Bolivia).

1610. Dendrocolaptes picirostris.

B. M.

Dendroplex picirostris, Lafr. O. Des Murs, Iconogr. Ornith. t. 51 .

S. America (New Granada). 
d. Nasica, Less. 1831.

1611. Dendrocolaptes longirostris.

B. $\mathrm{M}$.

Dendrocolaptes longirostris, $I l l$. Levaill. Promer. t. $24 . \quad$ Nasica nasalis et N. albicollis, Less. N. longirostris, $\operatorname{Pr} . B . \&$. Lafr.

S. America (Brazil).

1612. Dendrocolaptes flavigaster.

Xiphorhynchus flavigaster, $S w$.

Less. Nasica flavigaster, Lafr. Des Murs, Iconogr. Ornith. t. 52. Picolaptes obsoletus, p. ? G. R. Gr.

Central America (Mexico).

1613. Dendrocolaptes rufigula.

Nasica rufigula, Less.

S. America.

1614. Dendrocolaptes guttatus.

B. M.

Dendrocolaptes guttatus, Licht. Dendrocops guttatus, $\operatorname{Pr} . B$.

Dendrocolaptes (Orthocolaptes) guttatus, Less. Nasica guttatus, Lafr.

S. America (Brazil, Bolivia?)

1615. Dendrocolaptes pardalotus.

B. M.

Dendrucopus pardalotus, Vieill. Levaill. Promer. t. 30. Dendrocolaptes flimmeus, Licht. D. guttatus, p. G. R. Gr. Dendrocolaptes (Orthocolaptes) guttatus, p. Less. Dendrocops guttatus, p. $\operatorname{Pr}$. B. Nasica pardalotus, $\operatorname{Lafr}$.

S. America (Guiana, Brazil); Central America (Panama).

1616. Dendrocolaptes chunchotambo.

Dendrocolaptes chunchotambo, Tschudi, Fanna Per. t. 22, f. 1.

Dendrocops chunchotambo, $\operatorname{Pr}$. B. Nasica pardalotus, p.

Lafr.? N. chunchutambo, Lafr.

S. America (Peru). 
1617. Dendrocolaptes triangularis.

Dendrocolaptes triangularis, Lafr. Mag. de Zool. 1845. Ois. t. 52. Nasica triangularis, Lafr. Dendrocincla? triangularis G. R. Gr.

S. America (Bolivia).

1618. Dendrocolaptes guttatoldes.

Nasica guttatoides, Lafr. Rev. Zool. 1850, p. 587.

S. America (Bolivia).

1619. Dendrocolaptes multiguttatus.

Nasica multiguttatus, Dev. et O. Des Murs, Rev. Zool. 1850, p. 417 .

S. America (Brazil).

1620. Dendrocolaptes Beacperthuysit.

Nasica Beauperthysii, Pucher. et Lafr. Rev. Zool. 1850, p. 419.

S. America (Peru, River Amazou).

1621. Dendrocolaptes D'Orbignyanus.

B. $M$. Nasica D'Orbignyanus, Pucher. et Lafr. Rev. Zool. 1850, p. 420 .

S. America (Peru).

1622. Dendrocolaptes ocellatus.

B. MI. Dendrocolaptes ocellatus aut guttatus, Spix, Av. Bras. t. 91, f. 1. Picolaptes ocellatus, G. R. Gr. Nasica ocellatus, Lafr.

S. America (Brazil).

1623. Dexprocolaptes obsoletus.

B. 11 . Dendrocolaptes obsoletus, Ill. Picolaptes obsoletus, G. $R$. Gr. Nasica obsoletus, Lafr.

S. America (Brazil, Peru). 
1624. Dendrocolaptes susurrans.

Dendrocolaptes susurrans, Jard. Nasica susurrans, Lafr.

Island of Tobago.

1625. Dendrocolaptes'Bridgesii.

Nasica Bridgesii, Eyton, Contr. to Ornith. 1849.

S. America (Bolivia).

e. Xiphorhynchus, $S w .1827$.

1626. Dendrocolaptes trochilirostris.

B. M.

Dendrocolaptes trochilirostris, Licht. Berl. Trans. 1818, t. 3. Dendrocopus falcularius, Vieill. Gal. des Ois. t. 175. Xiphorhynchus trochilirostris et X. falcularius, $G . R$. Gr. Dendrocolaptes procurvus, Temm. $P l$. col. 28, f. 4. Xiphorhynchus procurvus, G. R. Gr.

S. America (Brazil).

1627. Dendrocolaptes procurvoides.

Xiphorhynchus procurvoides, Lafr. Rev. Zobl. 1850, p. 376.

S. America (Guiana).

1628. Dendrocolaptes Lafresnatanus.

Dendrocolaptes procurvus, $D^{\prime} O r b$. \& Lafr. D. Lafresnayanus, D'Orb. Voy. dans l'Amer. Mer. Ois. t. 55, f. 2. Xiphorhynchus Lafresnayanus, G.R. Gr.

S. America (Bolivia).

1629. Dendrocolaptes Pucheran.

Dendrocolaptes Pucheranii, Lafr. Xiphorhynchus Pucheranii, O. Des Murs, Iconogr. Ornith. t. 68.

S. America (New Granada).

1630. P DENDROCOLAPTES LEUCOGASTER.

Xiphorhynchus leucogaster, $S w$. Picolaptes obsoletus, p.? $G$. R. Gr. 
f. Picolaptes, Less. 1830.

Tripobrosus, $\mathrm{Cab} .1847$.

1631. Dendrocolaptes angustirostris.

B. $M$.

Dendrocopus angustirostris, Vieill. Dendrocolaptes bivittatus, Licht. Berl. Trans. 1820, t. 2, f. 2. Spix, Av. Bras. t. 90, f. 1. D. rufus, $\operatorname{Pr}$. Max. Dendrocolaptes (Xiphocolaptes) bivittatus et angustirostris, Less. Picolaptes coronatus, Less. P. tenuirostris, p. G. R. Gr. P. bivittata, G. R. Gr.

S. America (Paraguay, Bolivia).

1632. Dendrocolaptes squamatus.

B. $\mathrm{M}$.

Dendrocolaptes squamatus, Licht. Berl. Trans. 1820, t. 2, f. 1. Dendrocolaptes (Xiphocolaptes) squamatus, Less. Picolaptes squamatus, G. R. Gr.

S. America.

1633. Dendrocolaptes Wagleri.

Dendrocolaptes Wagleri, Spix, Av. Bras. t. 90, f. 2. Picolaptes Wagleri, $G$. $R$. Gr.

S. America (Brazil).

1634. Dendrocolaptes tenuirostris.

Dendrocolaptes tenuirostris, Licht. Spix, Av. Bras. t. 91, f. 2. D. gracilirostris, Steph. Dendrocolaptes (Xiphocolaptes) tenuirostris, Less. Picolaptes Spixii et P. guttata, Less. Cent. Zool. t.32. P. tenuirostris, G. R. Gr.

S. America (Brazil).

1635. Dendorocolaptes lacrymier.

B. M.

Dendroculaptes lacrymiger, $L$ ifr. O. Des Murs, Iconogr. Ornith. t. 70. Picolaptes lacrymiger, $P r . B$.

S. America (Venezuela, Brazil).

1636. Dendrocolaptes aFFinis.

Dendrocolaptes affinis, Lafr. D. angustirostris, Pr. B. Picolaptes affinis, G. R. Gr.

S. America. 
1637. Dendrocolaptes Souleyeti.

Dendrocolaptes Souleyetii, Lafr. O. Des Murs. Iconoyr. Ornith. t. 70. Picolaptes Souleyetii, Pr. B.

S. America.

1638. Dendrocolaptes lineaticeps.

Picolaptes lineaticeps, Lafi. Rev. Zool. 1850, p. 277.

S. America.

1639. Dendrocolaptes albolineatus.

Dendrocolaptes albolineatus, Lafr. Picolaptes albolineatus, G. R. Gr.

S. America (Columbia).

1640. Dendrocolaptes validirostris.

Picolaptes validirostris, Eyton.

S. America.

1641. Den DRocolaptes atripes.

B. M. Picolaptes atripes, Eyton.

S. America.

1642. Dendrocolaptes fuscus.

Dendrocopus fuscus, Vieill. Dendrocolaptes (Xiphocolaptes) fuscus, Less. Dendrocolaptes fuscus, $G . R$. Gr. Picolaptes tenuirostris, p. et P. fuscus, Lafr.

S. America.

1643. Dendrocolaptes maculiventris. Picolaptes maculiventris, Less.

S. America. 
g. Glyphorhynchus, $\operatorname{Pr}$. Max. 1831.

Sittacilla, Less. 1837.

Xenophasia, $S_{w} .183$ ?

Sphenorhynchus, $\operatorname{Pr}$. Max. 1831.

1644. DENDROCOLAPTES CUNEATUS.

B. M.

Dendrocolaptes cuneatus, Licht. Berl. Trans. 1818, t. 3, f. 2 . Mag. Zool. 1833. Ois. t. 17. Spix, Av. Bras. t. 91, f. 3. Sphenorhynchus aut Glyphorhynchus ruficaudus, $\operatorname{Pr}$. Max. G. cuneatus, G. R. Gr. Zenophasia platyrhyncha, Sw. Sittacilla — Less. Neops spirurus, Vieill. Levaill. Promer. t. 31, f. 1. Sittasomus flammulatus, Lafr.

S. America.

h. Dendrocincla, G. R. Gr. 1840.

Dryocopus, $\operatorname{Pr}$. Max. 1831.

1645. Dendrocolaptes turdinus.

B. M.

Dendrocolaptes turdinus, Licht. Berl. Trans. 1818, t. 2, f. 1. Dryocopus turdinus, $\operatorname{Pr}$. Max. Dendrocincla turdina, $G$. $R$. Gr.

S. America.

1646. Dendrocola Ptes Fumigatus.

B. M.

Dendrocolaptes fumigatus, Licht. Levaill. Promer. t. 28. Dendrocopus fuliginosus, Vieill. Dendrocincla fumigata, $G$. R. $G r$.

S. America.

1647. Dendrocolaptes merula.

Dendrocolaptes merula, Licht. Dendrocincla merula, $G . R$. Gr.

S. America.

1648. Dendrocolaptes Perrotit.

Dendrocolaptes Perrotii, Lafr. Mag. Zool. 1844. Ois. t. 54.

Dendrocincla Perrotii, G. R. Gr.

S. America (Columbia). 
1649. DENDROCOLAPTES ATRIRostris.

Dendrocolaptes atrirostris, D'Orb. \& Lafr. Voy. dans $I$ Amer. Mer. t. 54, f. 1. Dendrocincla atrirostris, G. R. Gr.

S. America (Bolivia).

\section{i. Dendrexetastes, Eyton, 1851.}

1650. Dendrocolaptes capitoides.

Dendrexetastes capitoïdes, Eyton.

S. America.

\section{Sittasomus.}

Sittasomus, Sw. 1827.

1651. Sittasomus erythacus.

B. M.

Dendrocolaptes erythacus, Licht. Berl. Trans. 1820, t. 1, f. 2 ,

3. D. zenops et D. sylviellus, Temm. Pl.col. 72,f. 1. Sittasomus sylviellus, $S w$. S. olivaceus, $\operatorname{Pr}$. Max. S. erythacus, G. R. Gr. S. Temminckii, Less. Synallaxis sylviellus, Cur: Kittl. Kupf.der Vög. t. 24, f. 2.

S. America (Brazil, Bolivia).

1652. Sittasomus sflvioides.

Sittasomus sylviöides, Lafr.

Central America (Mexico).

1653. Sittasomus amazonus.

Sittasomus amazonus, Deville \&. O. Des Murs.

S. America (River Amazon).

1654. SitTasomes griseus.

Sittasomus griseus, Jard.

W. Indies (Tobago). 
iii. Certhiane.

Certhianæ, Pr. B. $1831-38-49-50$; Sw. 1837 ; G. R. Gr. $1840-41-47 ; C a b .1847 ; B l .1849$.

Tichodromina? p. Sw. 1827.

Tichodrominæ, $\operatorname{Pr} . B .1849$.

Sittinæ, p. $S u .1831 ; B l .1849$.

\section{Certhia.}

Certhia, $L .1735$.

1655. Certhia familiaris.

B. M.

Certhia familiaris, Brehm. Naum. Vög. Deuts. 1826, v. t. 140. C. scandulaca, $P$ all. C. macrodactyla, C. septentrionaIis, C. brachydactyla, et C. megarhvnchos, Brehm. Yarr. Brit. $B$. 2 ed. ii. 165 , fig. $P l$. enl. 681 , f. 1 . Gould, B. of Eur. pl. 237.

Great Britain; Europe.

1656. Certhia Nattereri.

B. M.

Certhia Nattereri, Pr. B. C. Costæ, Parzud.

Central Europe.

1657. Certhia americana.

Certhia familiaris, Wils. Amer. Orn. pl. 8, f. 1. C. americana, Pr. B. Audub. B. of Amer. pl. 419.

N. America.

1658. Certhia nipalevsis.

B. M.

Certhia nipalensis, Hodgs. Gould, B. of Asia, pl. C. himalayana, $B l$.

India (Nepal).

1659. ? Certhia discolor.

Certhia discolor, $B l$.

India (Sikim). 
1660. Certhia himalayana.

B. M.

Certhia limalayana, Vig. Gould, B. of Asia, pl. . C. asiatica, $\$ w$.

India.

\section{Caulodromus.}

Caulodromus, G. R. Gr. 1847.

Rimator, $B l .1847$.

Merva, Hodys. 1847.

1661. Caulodrouus Gracei.

B. M.

Caulodromus Gracei, G. R. Gr. \&. Mitch. Gen. of B.p!.44, f. 2. Rimator malacoptilus, $B l$. Merva Jerdoni, Hodys. M:Clell. Calc. Journ. N. H. 1847, pl. 3, f. 2.

India.

\section{Salporis.}

Salpornis, G. R. Gr. 1847.

1662. Satpornis spilonota.

B. M.

Certhia spilonota, Franlkl. Salpornis spilonota, G. R. Gr. SMitch. Gen. of B. pl. 44, f. 1.

India.

\section{Tichodroma.}

Tichodroma, Ill. 1811.

Petrudroma, Vieill. 1816.

1663. Tichodroma muraria.

B. $\mathrm{M}$.

Certhia muraria, $L$. Tichorlroma muraria, $I l l$. T. phænicoptera, Temm. T. europæa, Steph. Petrodroma muraria, Vieill.

Europe.

1664. ? TICHODROMA NIPALENSIS.

B. M.

Tichodroma phænicoptera, $\nabla$. subbemalayana, Hodgs. ' $Г$. nipalensis, $\operatorname{lr}$. $B$. ' T. muraria; Hodgs. \&- $\boldsymbol{B} l$.

India (Nepal). 


\section{Climacteris.}

Climacteris, Temm. 1820.

1665. Climacteisis leucoph a. A.

B. M.

Certhia leucophæa et C. leucoptera, Lath. Vieill. Ois. dor. t. 127? Climacteris leucophas, Strickl. C. picumnus, Temm. Pl. col. 28, f. 1. Gould, B. of Austr. iv. pl. 98. Nectarinia picumnus, $C u v$.

Australia.

1666. Climacteris Bailloni.

B. M.

Petrodroma Bailloni, Vieill. N. Dict. d'Hist. Nat. xxvi. t. M, 33, f. 3. Climacteris scandens, Temm. Pl. col. 281, f. 2. Gould. B. of Austr. iv. pl. 93. Kittl. Kupf.V̈̈g. t. 6, f. 1. C. Bailloni, G.R. Gr. Nectarinia scandens, Cuv.

Australia.

1667. Climacteris erythrops.

B. M.

Climacteris erythrops, Gould, B. of Austr. iv. pl. 95.

Australia.

1668. Climacteris RUfa.

B. M.

Climacteris rufa, Gould, B. of Austr. iv. pl. 94.

Australia.

1669. Climacteris metandra.

Climacteris melanura, Gould, B. of Austr. iv, pl. 97.

Australia.

1670. Climacteris melanotus.

Climacteris melanotus, Gould, B. of Austr. iv. pl. 96.

167 1. Climacteris mysticalis.

B. M.

Meliphaga mysticalis, Temm. Pl. col.335, f. 2. (M. striolata?) Kittl. Kupf. Vög. 1. 6, f. 2. Climacteris mysticalis, G. R. Gr. C. striolata, Hartl. 
iv. SitTiNe.

Sittinæ, Pr. B. 1831-38-49-50; Sw. 1837; G. R. Gr. 1840 -41-46; Cab. 1847; Bl. 1849.

Anabatinæ, p. $S w .1837$.

Dendroculaptinæ, p. Cab. 1847.

\section{Sitta.}

a. Sitta, L. 1735 .

1672. SitTA EUROPAA.

B. M. Sitta europxa, L. Nils. Skandin. Fauna, t. . S. uralensis, Licht. S. europæa var. sibirica, Pall. S. sericea, Temm. S. asiatica, Gould, B. of Eur. pl. 236. S. septentrionalis, Brehm.

N. Europe.

1673. SitTA C.ESIA.

B. M

Sitta europæa, Leach. Sitta cæsia, Meyer. S. pinetorum, S. foliorum et S. adrena, Brehm. S. affinis, Blyth. Yarr. Brit. B. 2 ed. ii. 183, fig. European Nuthateh, Mont. Pl. enl. 623, f. 1. Gould, B. of Eur. pl. 234.

Great Britain; Medial and Southern Europe.

1674. SitTa syriaca.

B. $M$.

Sitta syriaca, Ehrenb. Pr. B. Fauna Ital. t. , f. . S. Nenmeyeri, Michahell. S. saxatilis, Schinz. S. rupestris, Temm. S. rufescens, Gould, B. of Eur. pl. 235.

East Europe and West Asia.

1675. Sitta Ruseilia.

Sitta roseilia, Pr. B. Consp. p. 227.

Japan.

1676. Sitta himatayexsis.

B. M.

Sitta himalayensis, Jard. \&. Selby, Ill. of Orn. pl. 146. Gould, B. of Asia, pl. S. indica, Burt.? S. himalayana, Bl.

S. nipalensis, Hodgs. S. vitticauda, James.

India (Nepal, Darjeeling). 
1677. Sitta castaneoventris.

B. M.

Sitta castaneoventris, Frankl. Jard. \& Selby, Ill. of Orn. pl. 145. S. castanea, Less. S. ferrugineoventris, Gould, B. of India. Asia, pl. 9. Guér. Icongr. t. 23, f. 3.

1678. Sitta cinnamoventris. Sitta cinnamoventris, $\boldsymbol{B} \boldsymbol{l}$. neoventris, Hodgs.

B. M. Gould, B. of Asia, pl. S. castaIndia (Nepal, Darjeeling).

1679. SitTA LEUCOPSIS.

Sitta leucopsis, Gould, B. of Asia, pl. 10. S. castanilia, Pr. B. MSS.

India (Himalayah Mountains).

1680. Sitta CaRolinensis.

Sitta carolinensis, Lath. B. M. B . Wils. Amer. Orn. pl. 2, f. 3. Audub. B. of Amer. pl. 152. S. jamaicensis, L.? Sloane, Jamaica, t. 254, f. 1 ? S. melanocephala, Vieill. Gal. des Ois. t. 71.

N. America.

1681. Sitta canadensis.

B. $M$.

Sitta canadensis, Lath. Pl. enl. 623 , f. 2 . S. varia, Wils. Amer. Orn. pl. 2, f. 4. S. stulta, Vieill. Audub. B. of Amer. pl. 105.

N. America.

1682. Sitta pusilla.

B. MI.

Sitta pusilla, Lath. Wils. Amer. Orn. pl. 15, f. 2. Audub. B. of Amer. pl. 105.

N. America.

1683. Sitta Pygman.

Sitta pygmæa, Vig. Beechey's Voy. Birds, pl. 4, f. 2. Audub. B. of Amer. pl. 419.

N.-W. America. 
b. Callisitta, Pr. B. 1850.

1684. Sitta formosa.

B. $M$.

Sitta formosa, Bl. Gould, B. of Asia, pl. 7. Callisitta formosa, $\operatorname{Pr} . B$.

India (Nepal, Sikim, Bootan).

c. Orthorhynchus, Horsf. 1820.

Dendrophila, $S w .1837$.

1685. Sitta frontalis.

B. M.

Sitta frontalis, Horsf. Sw. Zool. Illustr. pl. 2. S. velata, Temm. Pl. col. 72, f. 3 . Dendrophila frontalis, Sw. D. velata, Hartl.

Malayan Peninsula and Archipelago.

1686. ? Sitta CORallina.

B. M.

Sitta corallina, Hodgs.

India (Nepal).

1687. Sitta flavipes.

B. M.

Dendrophila flavipes, $S w$. D. gymnophrys, $\operatorname{Pr} . B$. D. azurea, Hartl. Sitta flavipes, G. R. Gr. \& Mitch. Gen. of B. pl. 45. S. azurea, Less. S. gymnophrys, Kuhl.

Malayan Archipelago.

\section{d. Sittella, $\$$ w. 1837.}

1688. Sitta chrtsoptera.

B. M.

Sitta chrysoptera, Lath. Gen. Syn. pl. 127. Sittella chrysoptera, Sw. Gould, B. of Austr. iv. pl. 101. Neops chrysoptera, Vieill.

Australia.

1689. Sitta pileata.

B. M.

Sittella pileata et S. melanocephala, Gould, B. of Austr. iv. pl. 104.

Australia. 
1690. Sitta leucocephala.

B. M.

Sittella leucocephala. Gould, B. of Austr. iv. pl. 102.

Australia.

1691. Sitta leucoptera.

B. M.

Sittella leucoptera, Gould, B. of Austr. iv. pl. 103.

Australia.

\section{Acanthisttita.}

Acanthisitta, Lafr. 1843.

1692. Acanteisitita longipes.

Motacilla longripes, Gm. Sylvia longipes, Lath. Acanthisitta

longipes, G. R. Gr. Zool. of Ereb. \&. Terr. Birds, pl. 3, f. 1.

New Zealand.

1693. Acanthisitita CHLORIS.

B. M.

Sitta chloris, Sparrm. Mus. Carls. pl. 33. Motacilla citrinella, Forst. Motacilla citrina, Gm. Sylvia citrina, Lath. Sitta punctata, Quoy \&. Gaim. Voy. de l'Astrol. t. 18, f. 1. Acanthisitta chloris, G. R. Gr. Zool. Ereb. \&. Terr. pl. 3, f. 1. A. tenuirostris, Lafr. Acanthiza tenuirostris, Lafr.

New Zealand.

\section{Xenops.}

a. Xenops, Hoff. 1811.

Neops, Vieill. 1816.

1694. Xenops aenibarbis.

B. M.

Xenops geniharbis, Ill. $P l$. col. 150, f. 1. X. Hoffmanseggi, Cuv. Levaill. Promer. t. 31, f. 2. Neops ruficauda, Vieill.

S. Anerica (Brazil).

1695. Xenops Rutilans. B. MI.

Xenops rutilans, Temm. Pl. col. 72, f. 1. X. rutilus, Licht. X. genibarbis et $\mathbf{X}$. aftisis, Sw. Zool. Illustr. t. 100 . Neops ruficauda, Vieill. Gal. des Ois. t. 170.

S. America (Brazil, Bolivia). 
1696. Xenops dentirostris.

Xenops dentirostris, $S w$.

S. America (Demerara?)

1697. Xenops Abeirlit.

Xenops Abeillii, Less.

S. America (— ?)

b. Anabazenops, Lafr. 1842 .

Anabasitta, Lafr. 1838.

1698. Xenops atricapillus.

B. $M$.

Anabates atricapillus, $\operatorname{Pr}$. Max. Sphenura superciliaris, Licht. Philydor superciliaris, Spix, Av. Bras. t. 73, f. 1. Anabates superciliaris, Cuv. Xenops Canivetii, Less. Cent. de Zool. t. 16. A. melanocephalus, Less.

S. America (Brazil).

1699. Xenops fuscus.

Xenops anabatoïdes, Temm. Pl. col. 150, f. 2. X. fuscus, Lafr. Sitta fusca, Vieill. Anabaxenops fuscus, Lafr. Sphenura albicollis, Licht.

S. America (Brazil).

1700. Xenofs rufosuperciliatus.

B. M.

Xenops rufosuperciliatus, Lafr. Mag. de Zool. 1832. Ois. t. 7.

Anabaxenops rufosuperciliatus, Hartl.

S. America (La Plata, Bolivia).

1701. Xenops LEucophrYs.

B. M.

Anabates leucophrys, Jard. \&. Selby, Illustr. of Orn. pl. 93.

S. America.

1702. Xenops Griseds.

Xenops griseus, Less. Anabates griseus, G. R. Gr.

S. America.

1703. Xenots RUFUS.

Xenops rufus, Less. Anabates rufus, G. R. Gr.

S. America. 


\section{c. Dendrodendron, G. R. Gr. 1842. \\ Dendrodramus, Gould, 1841. \\ Pygarrhichus, Licht. 1837.}

1704. XENops aldBogularis.

Dendrodromus leucostrnus, Gould, Voy. of Beagle, Birds, $p$ 27. D. albogularis, $L a f r$. Dromodendron leucosternum, $G$. R. Gr. Dendrocolaptes albogularis, King. Dendrocolaptes (Xiphocolaptes) albogularis, Less. Picolaptes albogularis, $G$. R. Gr. Pygarrhicus leucosternus, Cab.

S. America (Chili and Island of Chiloë).

\section{v. ORThonYCHINA.}

Orthonychinæ, G. R. Gr. 1840-47; Cab. 1847; Pr. B. 1849 -50 ,

Sittinæ, p. Pr. B. 1831.

Buphaginæ, p. Sw. 1837.

\section{Orthonyx.}

a. Orthonyx, Temm. 1820 .

1705. Orthonyx SPINICAUDA.

Orthonyx spinicauda, Temm. Pl. col. 428. O. maculatus,

Steph. O. Tenminckii, Vig. \&. Horsf.

Australia.

\section{b. Mohoua, Less. 1837.}

1706. ORthoNYx OCHRocephalus.

B. M.

Muscicapa ochrocephala, $G m$. M. chloris, Forst. Certhia heteroclitus, Quny. \& Gaim. Voy. de l'Astrol. t. 17, f. 1 . Orthonyx icterocephalus, Lafr. Mag. de Zool. 1840. Ois. t. 8. O. vchrocephalus, G. R. Gr. \& Mitch. Gen. of B. pl. 46. Mohoua hua, Less. M. ochrocephala, G. R. Gr. 
vi. Menurine.

Menurinæ, G. R. Gr. 1847 ; Cab. 1847; Pr. B. 1849-50.

Sylviana, p. Vigors, 1825.

Troglodytæen,$S w .1831-37$.

Troglodytinæ, G. R. Gr. 1840-41; Cab. 1847; Pr. B. 184950.

Laniinæ, p., Sylvinæ, p. et Penelopidæ, p. Pr. B. 1831.

Certhinæ, p. Pr. B. 1838.

Myiotherinæ, p. et Eriodorinæ, p. Cab. 1847.

Crateropodinæ, p. Sw. 1837.

Pittinæ, p. $\operatorname{Pr} . B .1850$.

\section{Menura.}

Menura, Davis, 1800.

Parkinsonius, Bechst.

170\%. Menura superba.

B. $M$.

Menura superba, Davis, Linn. Trans. vi. pl. 22. Gould, B. of Austr. iii. pl. 14. M. lyra, Shaw. M. novæ hollandiæ, Lath. M. vulgaris, Flem. M. paradisea, Sw. M. lyrata, Less. Vieill. Ois. dor. t. 14, 15. Gal. des Ois. t. 192. Parkinsonius mirabilis, Bechst. Megapodius menura, Wagl.

Australia.

1708. Menura Alberti.

Menura Alberti, Gould, B. of Austr. Suppl. pl. Australia.

\section{RHINOCRYPTA.}

Rhinocrypta, G. R. Gr. 1841.

Rhinomya, D'Orb. \&. J. Genff. 1832.

1709. Rhinocrypta lanceolata.

B. M.

Rhinomya lanceulata, D'Orb. \& Lafr. Voy. dans l'Amer. Mer. Ois. t. 7, f. 1. Mag. de Zool. 1832. Ois. t. 3. Compl. Buff.

Ois. t. 7, f. 1, 2. Rlinocrypta lanceolata, G. R. Gr.

S. America (Patagonia). 


\section{Pteroptochos.}

a. Hylactes, King, 1839 .

Megalonyx, Less. 1830.

Pteroptochos, p. Kittl.

1710. Pteroptochos Tarnet.

B. M.

Hylactes Tarnii, King. Megalonyx ruficeps, D'Orb. \& Lafr. Voy. dans l'Amer. Mer. Ois. t. 8, f. 1.

S. America (Chili).

1711. Pteroptochos megapodius.

B. $M$.

Pteroptochos megapodius, Kittl. Vög. von Chili, t. 4. Kupf. der Vög. t. 16, f. 1. Megalonyx rufus, Less. Cent. de Zool. t. 66. Leptonyx macropus, Sw. Zool. Illustr. n. s. pl. 117. Hylactes megapodius, G. R. Gr. H. rufus, Cab.

S. America (Chili).

b. Pteroptochos, Kittl. 1830.

Leptonyx, Sw. 1832.

1712. Pteroptochos albicollis.

B. M.

Pteroptochos albicollis, Kittl. Vög. von Chili, t. 3. Kupf. der Vög. t. 16, f. 2. Megalonyx medius, Less. Illustr. de Zool. t. 60. M. albicollis, D'Orb. \& Lafr. Leptonyx albicollis, D'Orb. \& Lafr. Voy. dans l'Amer. Mer. Ois. t. 8, f. 2.

S. America (Chili).

1713. Pteroptochos rubecula.

B. $M$.

Pteroptochos rubecula, Kittl.Vög. von Chili, t. 2. Megalonyx vel Leptonşx rubecula rel rufogularis, D'Orb. \& Lafr. Voy.

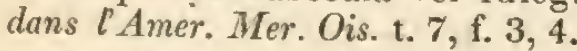

S. America (Chili).

c. Merulaxis, Less. 1830.

Platyurus, $S w .1837$.

Sarochalinus, Cab. 1847. 
1714. Pteroptochos ater.

B. M.

Merulaxis ater, Less. Cent. de Zool.t. 30. M. rutilus, Less. Malacorhynchus cristatellus, Menetr. Mem. Acad. St. Petersb. 1831, t. 12. Platyurus corniculatus, Sw. B. of Braz. pl. 55, 56. Sarochalinus ater, $\mathrm{Cab}$.

S. America (Brazil).

1715. Pteroptochos RHYNCOLOPHUS.

Myiothera rhynolopha, Pr. Max. Malacorhynchus rhynolophus, Menetr. Sarochalinus rhynolophus, $C a b$. Pteroptochos rhyncolophus, G. R. Gr.

S. America (Brazil).

d. Triptorhinus, Cab. 1847.

1716. Pteroptochos paradoxus.

B. M.

Troglodytes paradoxus, Kittl. Vög. von Chili, 1.5. Platyurus lepturus, Sw. Leptonyx paradoxus, Lafr. Malacorhynchus chilensis, Menetr. Sylviaxis paradoxus, Less. Pteroptochos chilensis, G.R. Gr. Triptorhinus paradoxus, $\mathrm{Cab}$.

S. America (Chili).

1717. Pteroptochos orthonyx.

Merulaxis orthonyx, Lafr. Mag. de Zool. 1844, t. 53. Triptorhinus orthonyx, $C a b$. Pteroptochos orthonyx, G.R. Gr.

S. America (Columbia).

e. Scytalopus, Gould, 1836 .

Sylviaxis, Less. 1840.

1718. Pteroptochos magellanicus.

B. $M$.

Motacilla magellanica, Gm. Sylria magellanica, Lath. S. obscura, King. Pteroptochos magellanicus, G. R. Gr. Scytalopus fuscus, Gould. Jard. \& Selby, Illustr. of Ornith. n. s., pl. 19. S. magellanicus, G.R. Gr. Conirostrum fuliginosum et Sylviaxis magellanicus, Less.

S. America (Patagonia). 
1719. ? Pteroptochos Niger.

Platyurus niger, Sw. Scytalopus niger, Cab. Voy. au Pole Sud, Ois. t. 19, f. 5 ?

S. America (Chiii).

1720. Pteroptochos SPelunce.

Malacorhynchus speluncæ, Menetr. Mem. de l'Acad. St. Petersb. 1835, t. 13, f. 1. Pteroptochos speluncæ, G. R. Gr.

S. America (Brazil).

\section{Pteroptochos senilis.}

Merulaxis senilis, Lafr. Pteroptochos senilis, G. R. Gr.

S. America.

1722. Pteroptochos indigotica.

Myiothera indigotica, Licht. Malacorhynchus albiventris et M. indigoticus, Menetr. Mem. de l'Acad. St. Petersb. 1835, t. 13, f. 2. Scytalopus albogularis, Gould. S. indigoticus, $C a b$. S. undulatus, Jard. \& Selby. Pteroptochus indigoticus et P. albiventris, G. R. Gr.

S. America.

1723. Pteroptochos analis.

Merulaxis analis, Lafr. Pteroptochos analis, G. R. Gr.

S. America.

1724. Pteroptochos rufocapillus.

Megalonyx rufocapillus, Less. Pteroptochos rufocapillus, $G$. R. Gr.

Island of Chiloë.

1725. Pteroptochos nanus.

Megalonyx nanus, Less. Pteroptochos nanus, G. R. Gr.

Island of Chiloë. 
1726. Pteroptochos squamiger.

B. M.

Merulaxis squamiger, Lafr. Pteroptochos squamiger, $G . R$. Gr.

S. America (New Granada).

1727. Pteroptochos acutirostris.

Pteroptochus vel Scytalopus acutirostris, Tschudi.

S. America (Peru).

1728. Pteroptochos femoralis.

Pteroptochus vel Scytalopus femoralis, Tschudi.

S. America (Peru).

1729. Pteroptochos griseicollis.

Merulaxis griseicollis, Lafr. Pteroptochos griseicollis, $G$. R. $G r$.

S. America (New Granada).

1730. Preroptochos guttatus.

Sylviaxis guttatus, Less. Pteroptochos? guttatus, G. R. Gr.

S. America.

\section{Cyphorhinus.}

Cyphorhinus, Cab. 1844.

1731. Cxphorhinds musicus.

B. M.

Formicarius musicus, Bodd. Pl. enl. 706, f. 1. Turdus cantans, $G m$. T. arada, Lath. Platyurus rubecola, $S w . B$. of Braz. pl. 14. Cyphorhinus musicus et C. carinatus, $G$. $\mathscr{R}$. Gr. C. cantans, Cab. Troglodytes arada, D'Orb. \& Lafr.

S. America (Guiana, Bolivia).

1732. CyphorhinUs AFFinis.

Platyurus affinis, Sw. B. of Braz.pl.57. Cyphorhinus thoracicus, Tschudi, Fauna Per. t. 16, f. 1.

S. America (Brazil, Peru). 
1733. Cyphorhinus leucophrys.

Troglodytes leucophrys, Tschudi. Cyphorhinus leucophrys, $\mathrm{Cab}$.

S. America (Peru).

1734. Cyphorhinus leucostictus.

Troglodytes leucostictus, Licht. Cyphorhinus leucostictus, Cab.

S. America (Guiana).

\section{Tesia.}

a. Tesia, 1837; Anura, 1841, et Pnoëpyga, 1845, Hodgs.

Micrura, Gould, 1837.

Aipunemia, $S w .1837$ ?

Apynemia, Strickl. 1844?

B. M.

1735. Tesia squamata.

Tesia albiventer, Hodgs. Pnoëpyga (Tesia) albiventer et $\mathrm{P}$. (T.) rufiventer, Hodgs. Micrura squamata, Gould, Icones Av. pl. . Pnoëpyga squamata, $B l$.

India (Nepal).

1736. TESLA CONCOLOR.

B. M.

T'esia concolor, Hodgs. Pnoëpyga (Tesia) concolor, Hodgs. Pnoëpyga unicolor, Hodgs. P. squamata, p. Bl.

India (Nepal).

1737. Tesia pusilita.

B. M.

Pnoëpyga (Tesia) pusillus, Hodgs. Pnoëpyga pusilla, $B l$.

India (Nepal).

1738. Tesia caudata.

Tesia caudata et Pnoëpyga caudata, $B l$.

India (Sikim). 
b. Oligura, Hodgs. 1845.

1739. Tesia castaneocoronata.

B. M.

Sylvia? castaneocoronata, Burt. Tesia flaviventris, Oligura (Tesia) flaviventer, et O. flaviventer, Hodgs. Tesia castaneocoronata, G. R. Gr.\& Mitch. Gen. of B. pl. 47, upp. fig.

India (Nepal, Sikim).

1740. Tesia cyaniventer.

B. M.

Tesia cyaniventer, Oligura (Tesia) cyaniventer, et Oligura cyaniventer, Hodgs. Tesia auriceps, Hodgs. Saxicola? oliracea, Mc Clell.

India (Nepal, Sikim, Assam).

$$
\text { c. Microura, Pr. B. } 1850 \text { (nec Gould). }
$$

1741. Tesia?

Microura squamata (Gould?), Pr. B. Consp. p. 258.

Java.

1742. Tesia? seperciliaris.

Microura superciliaris, Miell. Pr. B. Consp. p. 258. Java.

\section{Sylvietta.}

Sylvietta, Lafr. 1839.

Oligura, 1845, et Trochlotides, Rüpp.

1743. Sylvietta micrurus.

B. M.

Troglolytes micrurus et $\mathrm{T}$. brevicaudatus, et Oligura micrurus, Riipp. Fauna Abyss. t. 41, f. 1.

N.-E. Africa.

1744. Sylinietta RUfescens.

B. M.

Dicæum rufescens, Vieill. Levaill. Ois. d'Afr. t. 135. Sylvietta crombec, Lafr. Drymoica? rufescens, G. R. Gr. Oligura rufescens, $\mathrm{Cab}$.

S. Africa. 
1745. Sylvietta? Brachyura.

Sylvia brachyura, Vieill. Levaill. Ois. d'Afr. t. 125. Phyl-

lopnenste chloris, Boie. Sylvietta brachyura, Lafr. Voy. en Abyss. 1839-43, t. 6. Drymoica? brachyura, G. R. Gr.

W. and S. Africa.

1746. Sylvietta ICTEROPYGialis.

Sylvietta icteropygialis, Laf $r$.

S. Africa.

1747. Sylvietta lutescens.

Sylvietta lutescens, Less.

W. Africa (Gambia).

\section{Tatare.}

Tatare, Less. 1831.

Tatarea, Reichenb. 1849.

1748. Tatahe longirostris.

B. M.

Sitta ototare, Less. Voy. de la Coq. Ois. t. 20, f. 2. Turdus longirostris, Gm. Oriolus musæ. Forst. Tatare otaitiensis et T. fuscus, Less. $\quad$ T. otatare, $\operatorname{Pr} . B$. T. longirostris, G. $R$. Gr. Society Islands.

1749. TAtare LUSCiniUs.

'I'hryothorus luscinius, Quoy \& Gaim. Voy. de l'Astrol. Ois. t. 5, f. 2. Tatare luscinius, $P r . B$. T. longirostris, p. G. R. Gr. Voy. au Pole Sud, Ois. t. 20, f. 5.

Islands of the South Sea.

\section{Troglodytes.}

a. Troglodytes, Vieill. 1807. Anorthura, Renn. 1831.

1750. TroglodYtes PARVulus.

B. M.

Motacilla troglodytes, Gm. Sylvia troglodytes, Lath. Troglodytes parvulus, Koch. Naum. Vog. Deuts. ii. t. 83, f. 4, (1822). Troglodytes europæus, Cuv. Leach. T. vulgaris, Flem. T. regulus, Meyer. T. troglodytes (Cuv.), Schleg. T. punctatus, (K̈och), Boie. T. domesticus et T. sylvestris, Brehm. Anorthura communis, Renn. A. troglodytes, Macgill. Yarr. Brit. B. 2 ed. ii. 169.

Great Britain; Europe. 
1751. Troglodytes Fumigatus.

Troglodytes fumigatus et T. europæus, Temm. Japan.

1752. Troglodytes subhemalayants.

Troglodytes subhemalayanus et T. nipalensis, Hodgs.

B. M.

India (Nepal).

1753. Troglodytes punctatus.

Troglodytes punctatus, $B l$.

India (Darjeeling).

1754. Troglodytes hyemalis.

Troglodytes hyemalis, Vieill. Audub. B. of Amer. pl. 360, f. $1,2,3$. T. parvulus, var. americanus, Licht. Sylvia troglodytes, Wils. Amer. Orn. pl. 8, f. 6.

N. and N.-W. America; S. America (Brazil, La Plata).

1755. TROGLODYTES AMERICANUS.

Troglodytes americanus, $A u d u b$. B. of Amer. pl. 179. T. sylvestris, Gamb.

N. America.

1756. Troglodytes Parkmanit.

Troglodytes Parkmanii, Audub.

N.-W. Annerica.

1757. Trog LODYTES BREVIROSTRIS.

Troglodytes brevirostris, Nutt. Audub. B. of Amer. pl. 175.

N. America (Texas, S. Carolina).

1758. Troglodytes adon.

Troglodytes ædon, Vieill. T. furvus, p. Licht. Sylvia domestica, Wils. Amer. Orn. pl. 8, f. 3. Audub. B. of Amer. pl. 83.

N. and N.-W. America.

1759. Trog lodytes Furtus.

B. M.

Motacilla furva, Gm. Brown, Zool. Illustr. pl. 18. Sylvia furva, Lath. Troglodytes furrus, Licht. T. americana, Cuv. Gal. des Ois. t. 167.

N. and S. America (Bolivia). 
1760. Troglodytes hornensis.

Troglodytes hornensis, Less. 'T. magellanicus, Gould.

S. America.

1761. Troglodytes platensis.

B. M.

Sylvia platensis, Lath. $\quad P l$. enl. 730, f. 2 . Troglodytes platensis, Vieill. T. musculus, Licht. T. hyemalis et T. fulvus, D'Orb. \& Lafr. Thryothorus platensis, Pr. Max.

S. America (Brazil, Paraguay).

1762. Troglodytes chilensis.

Troglodytes chilensis, Less. T. guereza, Cuv.

S. America.

1763. Troglodytes $\mathbb{E Q U I N O C T I A L I S . ~}$

B. M.

Troglodytes æquinoctialis, $S w$. B. of Bras. pl. 13.

S. America (Brazil).

1764. Troglodytes tecellatus.

Troglodytes tecellata, $D^{\prime} O r b$. \& Lafr.

S. America (Peru).

1765. Troglodytes pallidus.

Troglorlytes pallida, D'Orb. \& Laf'r.

S. America (Patagonia).

1766. Troglonytes guarayanus.

Trogludytes guarayana, $D^{\prime} O r b$. \& Lafr.

S. America (Bolivia).

1767. Troglodytes MuRinus.

Troglodytes murinus, Less.

S. America (Peru).

1768. Trogiodytes audax.

Troglodytes audax, Tschudi.

S. America (Peru). 
1769. Troglodytes rufulus.

Troglodytes rufulus, Cab.

S. America (Guiana).

b. Thryothorus, Vieill. 1816.

1770. Troglodytes ludovicianus.

Sylvia ludoviciana, Lath. (150). Pl. enl. 730, f. L. Certhia caroliniana, Wils. Amer. Om. pl. 12, f. 3. Thryothorus littoralis, Vieill. T. ludovicianus, Steph. Troglodytes ludovicianus, Licht. Audub. B. of Amer. pl. 78.

N. America.

1771. Troglodytes arundinaceus.

B. M.

Certhia palustris, Wils. Amer. Orn. pl. 12, f. 4. Troglodytes arundinaceus, Vieill. Ois. de l'Amer. Septr.t. 108. T. palustris, Licht. Thryothorus arundiceus, Vieill. T. palustris, Pr. B. Audub. B. of Amer. pl. 100.

S. America.

1772. ?Troglodytes Eidouxi.

Thryothorus Eidouxi, Pr.B. Voy. au Pole Sud, Ois. t. 19, f. 6. Troglodytes, No. 15. G.R. Gr.

America.

1773. Troglodytes Bewickir.

'Troglodytes Bewicki, Audub. B. of Amer. pl. 18. T. spilurus, Vig. Beechey's Voy. Zool. pl. 4, f. 1. Thryothorus Bewicki, Pr. B.

N. and N.-W. America.

1774. Troglodytes steldaris.

Troglodytes stellaris, Licht.

Carolina?

1775. Troglodytes maculipectus.

B. $M$.

Thryothorus maculipectus, Lafr. 'Troglodytes maculipectus, G. R. Gr.

Central America (Mexico, Honduras). 
1776. Troglodytes leucotis.

Thryothorus leucotis, Lafr. Troglodytes leucotis, G. R. Gr.

S. America (Columbia? Mexico?).

1777. Troglodytes rufalbus. B. M.

Thryothorus rufalbus, Lafr. Troglodytes rufalbus, G. R. Gr. Central America (Mexico).

1778. Troglodytes leveogaster.

Troglodytes leucogastra, Gould. Troglodytes (Thryothorus) leucogastra, Lafr.

Central America (Mexico).

1779. Trog lodytes meXicanus.

B. M.

Thryothorus mexicanus, Sw. Zool. Illustr. n. s. pl. . T. guttulatus, Lafr. Troglodytes guttatus, Hartl. T, albicollis, Cuv. T. mexicanus, G.R. $G r$.

Central America (Mexico).

1780. TROGLODYTES ERYTHROPHTHALMUS.

Troglodytes erythrophthalmus, Licht.

Central America (Mexico).

1781. Troglodytes DeFLEXUS.

Troglodytes deflexus, Licht.

Central America (Mexico).

1782. Troglodytes albindCha.

Troglodytes albinucha, Cabot.

Central America.

1783. Troglodytes rutilus.

B. M.

Thryothorus rutilus, Vieill. Troglodytes rutilans, Sw.B. of Braz. pl. 15. T. rutilus, G. R. Gr.

S. America. 
1784. Troglodytes melanos.

Thryothorus melanos, Vieill. Troglodytes melanos, G. R. Gr. S. America.

1785. Troglodytes polyglottus.

Thryothorus polyglottus, Vieill. Troglodytes polyglottus, $G$. $R$. Gr. T. omnisomus, Licht.

S. America (Paraguay).

1786. Troglodytes coraya.

B. $M$.

Turdus coraya, Gm. Pl. enl. 701, f. 1. Sphenura coraya, Licht. Myiothera coraya, Spix, Av. Bras. t. 73, f. 2. Troglodytes coraya, G. R. Gr. Thriothorus coraya, Hartl.

S. America (Brazil).

1787. Troglodytes modulator.

Thryothorus modulator, D'Orb. Troglodytes coraya, D'Orb. \&. Lafr.

S. America (Bolivia).

1788. Troglodytes fasciativentris.

Thryothorus fasciatoventris, Lafr. Troglodytes fasciativentris, G. R. Gr.

S. America (New Granada).

1789. Troglodites rosaceus.

Thryothorus rosaceus, Less. Vieill. Ois. de l'Amer. Sept. t. 107? Troglodytes rosaceus, G. R. Gr.

S. America (La Plata, Chili).

1790. Troglodytes genibarbis.

Thryothorus genibarbis, $S w$. Troglodytes genibarbis, G. $R$. $G r$.

S. America (Brazil).

1791. Troglodytes striatulus.

Thryothorus striatulus, Lafr. Troglodstes striatulus, G. R. Gr.

S. America (New Granada). 
1792. Troglonytes interscapularis.

Troglodytes interscapularis, Licht. Thryothorns interscapularis, $\operatorname{Pr} . B$.

S. America.

1793. Troglody'tes LoNgirostris.

B. M.

Thryothorus longirostris, Vieill. Troglodytes longirostris et T. striolatus, G.R. Gr. Gal. des Ois. t. 168. T. striolatus, Sw. B. of Braz. pl. 16. Campylorhynchus striolatus, Spix, Av. Bras. t. 79, f. 2 .

S. America (Brazil).

1794. Troglodytes alBipectus.

'Thryothorus albipectus, $C a b$.

S.America (Guiana).

c. Salpinctes, Cab. 1847.

1795. Troglodites obsoletes.

Troglodytes obsoletus, Say. T. latifasciatus, Licht. Myiothera vel Thryothorus obsoletus, $\operatorname{Pr}$. B. Amer. Orn. pl. 1, f. 2. Audub. B. of Amer. pl. 360, f. 4. Salpinctes obsoletus, Cab.

N. W. America.

1796. Trog LODYTES MEXICANUS.

Thryothorns mexicanus, $S w$. Troglodytes mexicanus, $G . R$.

$G r$. T. murarius, Licht. Salpinctes mexicanus, Cab.

Central America.

d. Ramphocænus, Vieill. 1818.

Acontistes, Sundev. 1835.

Scolopacinus, $\operatorname{Pr}, B .1837$.

1797. Troglodxtes melanurus.

B. M.

Ramphocenus melanurus, Vieill. Gal. des Ois. t. 128. Troglodytes rectirostris, Sw. Zool. Illustr. pl. 140. T. gladiator, $\operatorname{Pr}$. B. Thriothorus? rectirostris, Steph.

S. America (Brazil). 
1798. Troglodytes Rufiventris.

Scolupacinus rufirentris, $P r$. B. Ramphocænus rufirentris, G. R. Gr. \& Mitch. Gen. of B. pl. 47, low. fig.

S. America.

1799. Troglodytes trinitatis.

Ramphocænus trinitatis, Less.

Antilles.

1800. Troglodytes viridis.

Ramphocænus viridis, Less.

S. America (Brazil).

\section{Chamea.}

Chamæa, Gamb. 1847.

1801. Cham

Parus fasciatus, Gamb. Journ. Acad. Nat. Sc. Philad. 1845, pI. 8, f. 5. Chamæa fasciata, Gamb.

N.-W. America.

\section{Campylorhynchus.}

a. Campylorhynchus, Spix, 1824.

Cichla, Wagl. 1827.

Ramphocinclus, Lafr. 1843. Pr. B. 1850.

L'Herminierus, Less.

1802. Campylorhynchus varlegatus.

B. M.

Turdus variegatus, Gm. T. scolopaceus, Licht. Campylorhynchus scolopaceus, Spix, Av. Bras. t. 79, f. 1. C. variegatus, G. R. Gr. Opetiorhynchus turdinus, Pr. Max.? Ramphocinclus tremulus, Lafr. R. variegatus, $P r . B$. Herminierus guadelupensis et $\mathbf{H}$. infaustus, Less. Picolaptes scolopaceus, Lafr. Mag. de Zool. 1833. Ois. t. 46. Formicarius tremulus, G. R. Gr. Anumbius scolopaceus, D'Orb. Troglodytes longirostris, p. Less.

S. America (Brazil). 
1803. Campylorhynchus gutturalis.

Thryothorus gutturalis, Less. Ramphocinclus gutturalis, Lafr. Formicarius gutturalis et Campylorhynchus gutturalis, G. R. Gr.

W. Indies.

1804. CamptlorhynchUS BrachyURUS.

Turdus brachyurus, Vieill. Ramphocinclus brachyurus, Lafr. Formicarius brachyurus et Campylorhynchus brachyurus, $G$. R. Gr.

W. Indies (Guadeloupe).

b. Campylorhynchus, $\operatorname{Pr} . B .1850$.

1805. Campylorhynchus NuChalis.

Campylorhynchus nuchalis, $\mathrm{Cab}$.

S. America (Venezuela).

1806. Campylorhynchus zonatus.

B. M.

Picolaptes zonatus, Less. Cent. de Zool. t. 70. Campylorhynchus zonatus, Lafr.

S. America (California).

1807. Campylorhynchus zonatoides.

Campylorhynchus zonatoïdes, Lafr.

S. America.

1808. CAMpylorhynchus Megalopterus.

Campylorhynchus megalopterus, Lafr. O. Des Murs, Iconogr. Ornith. pl. 54.

Central America (Mexico).

1809. Camprlorhy nchus brtenneicapillus.

B. M.

Picolaptes brunneicapillus, Lafr. Mag. de Zool. 1833. Ois. t.

54. Campylorhynchus brunneicapillus, Lafr.

S. America. 
1810. Campromhynchus gettatus.

Thryothorus guttatus, Gould. Campylorhynchus guttatus, Lafr.

Central America (Mexico).

1811. Campylorhynchus capistratus.

Picolaptes capistratus, Less. Campylorhynchus capistratus, G.R.Gr. C. rufinucha, Lafr. O. Des Murs, Iconogr. Ornith. pl. 63 .

Central America (Mexico).

1812. Campylorhynchus brevirostris.

Campylorhynchus brevirostris, G. R. Gr.

S. America (New Granada).

1813. Campylorhynchus cinnamomeds.

Picolaptes cinnamomeus, Less. Campylorhynchus cinnamomeus, Lafr.

S. America (Ecuador).

1814. Campylorhynchus pallescexs.

Campylorhynchus pallescens, Lafr.

S America (Mexico?).

1815. Camprlorhynchus UNicolor.

Campylorhynchus unicolor, Lafr.

S. America.

1816. Camprorirynchus UNicolorordes.

Campylorhynchus unicoloroïdes, G.R. Gr.

S. America (Bolivia). 


\section{Tribe 3. DENTIROSTRES.}

Passeres, p. Linn. 1735 ; Mahr. 1752.

Picæ, p. Mahr. 1752.

Accipitres, p. Linn. 1760.

Plumipedes, p. Schaff. 1774.

Fissipedes anisodactyles - Conico-convexirostres, p. - F. a. conicosubulirostres, p. Schaff. 1774.

Anomalipedes, p. Schaff. 1774.

Scutipedes, p.-Negliges, p.-Chanteurs, p. Scop. 1777.

Crenirostres-Conirostres, p.-Subulirostres, Cuv. 1799-1800.

Gregarii, p.-Canori-Passerini, p.-Serrati, Illiger, 1811.

Canori-Passerini-Coraces, p. Temm. 1815.

Egithali-Pericalles, p.-Textores, p. - Baccivori, p.-Myiotheres -Colluriones-Canori-Anerpontes, p.-Antriades, Vieill. 1816.

Dentirostres-Conirostres, p. Cuv. 1817.

Omnivores, p.-Insectivores-Granivores, p. Temm.1820.

Plereoramfi, p.-Ifanti, p. - Euristomi, p.-Piezoramfi, p.-Egitali -Rafioramfi-Metrioramfi, p.-Collurioni, Ranz. 1823.

Dentirostres-Conirostres, p.-Scansores, p. Vigors, 1826.

Volucres, p.-V. Oscines, Sundev. 1835.

Oscines, p. Keys. \& Bl. 1840.

Excurtrices, p.-Cantatrices, Macgill. 1840.

Oscines-Clamatores, p. Cabanis, 1847.

\section{LUSCINID无.}

Luscinidæ, G. R. Gr. 1841-48.

Sylviadæ, Vig. 1825; Sw. 1827-31-37; G. R. Gr. $1840 ;$ Cab. 1847.

Sylvila, $\operatorname{Pr} . B .1831$.

Pipridæ, p.; Meliphagidæ, p. Vig. 1825.

Motacillidæ, Gr. 1842;Bl.1849; Pr. B. 1849-50.

Trochilidæ, p. 1831; Turdidæ, p. $1849-50 . ;$ Menuridæ, p. $1849-$ 50, $\operatorname{Pr} . B$.

Rhænemididæ, p.; Sylvicolidæ, Cab. 1847.

Paridæ, Pr. B. 1831-49-50; Selys, 1842.

Sphenuridæ, p. ; et Brachyuridæ, p. Bl. 1849.

Cantores, p.; Parides; Alaudides, p.; Timalides, p.; Anabates, p. ; Certhiades, p. Sundev. 1835. 


\section{i. Malurine.}

Malurinæ, G. R. Gr. 1840-41-48; Cab. 1847; Pr. B. 184950.

Sylviana, p. et Motacillina, p. Vig. 1825.

Turdinæ, p. et Sylvinæ, p. Pr. B. 1831.

Crateropodinæ, p. Sw. 1837.

Calamoherpinæ, p. Pr. B. 1850.

\section{Orthotomus.}

Orthotomus, Horsf. 1820.

Edela, Less. 1830.

1817. Orthotomus Longicaudus.

B. MI. Motacilla longicauda et M. sutoria, Gm. Sylvia guzurata, Lath. Orthotomus longicaudus, $B l$. O. Bennettii et $O$. Lingo, Sykes. O. sutoria et O. patia, Hodgs. O. sphenurus, Swo. Malurus longicaudus, Steph. Sylria ruficapilla, Hutt. Cent. de Zool. f. 71. Mag. de Zool. 1836, t. 52, 53.

India (Nepal, Madras); Ceylon; Malayan Peninsula and Archipelago.

1818. Orthotomus cineraceds.

Orthotomus cineraceus, $\boldsymbol{B l}$.

Malacca.

1819. Orthotomus Sepium.

B. M. Orthotomus sepium, Horsf. Pl. col. 599, f. 1. Mag. de Zool. 1836. Ois. t. 58.

Java.

1820. Orthotomos cucullatus.

Orthotomus cucullatus, Temm. $\mathrm{Pl}$. col. 599, f. 3 .

Java and Sumatra.

1821. Orthotomes atrog Ularis. Orthotomus atrogularis, Temm. Pl. col. (with t. 599).

Malacca and Borneo. 
1822. ORthotonus EDELA. B. M.

Orthotoma edela, Temm. $P l$. col. 599, f. 2. Edela ruficeps,

Less. Cent. de Zool. t. 71. Motacilla sepium, Raff.

Malayan Peninsula and Archipelago.

1823. ORthotomos SERICEUS.

Orthotomus sericeus, Temm.

Borneo.

1824. Orthotomus (?) Longirostris.

Orthotomus longirostris, $S w$.

S.-W. Coast of Australia (?)

2. Prinia.

\section{Prinia, 1820.}

Daseocharis, Cab. 1851.

1825. Prinia familiaris.

B. M.

Prinia familiaris, Horsf. Zool. Res. pl. . Orthotomus prinia, Temm. Daseocharis familiaris, Cab. Sw. Zool. Illustr. n. s. pl. 97.

Java.

1826. Prinia olivacea.

Motacilla olivacea, Raff. Prinia olivacea, $B l$.

Sumatra.

1827. Prinia Hodgsoxi.

Prinia Hodgsoni, $B l$. P. gracilis, Jerd.

B. M

India (Nepal, Madras).

1828. Prinia flaviventris.

Orthotomus flaviventris, Deless. Prinia flaviventris, $B l$.

India and Malayan Peninsula. 
1829. Prinia socialis.

Prinia socialis, Sykes.

B. MI

India (Deccan).

1830. Prinia Stewartil.

Prinia Stewartii, $B l$. Sylvia kalaphutki, B. Ham. MSS.

India (Bengal).

1831. Prinia gracilis.

Prinia gracilis, Frankl.

India.

1832. Prinia rufescens.

Prinia rufescens, $\boldsymbol{B l}$.

India (Arakan).

1833. Prinia pileata.

Prinia pileata, $B l$.

India.

1834. Prinia icterica.

Prinia icterica, Strickl.

Fernando Po.

3. Drimoica.

a. Drymoica, Swains. 1827.

Drymœea, Cab. 1851.

1835. Drymoica maculosa.

B. M.

Motacilla maculosa, Bodd. Pl. enl. 752, f. 2. M. macroura, Gm. M. macroura, var. ocellata, Burch. Drymoica maculosa, G. R. Gr. Sylvia macroura, Lath. Curruca macroura, Cuv. Malurus capensis, Steph. Anabates macrourus, Licht. Drymœea macroura, Cab. Levaill. Ois. de lAfr. t. 129, 130, f. 1 .

S. Africa. 
1836. Drimoica Capensis.

B. M.

Drymoica capensis, Smith, Ill. Zool. S. Afr. pl. 76, f. 1.

S. Africa.

1837. Drymoica substriata.

B. $M$.

Drymoica substriata, Smith, Ill. Zool. S. Afr. pl. 72, f. 1 .

S. Africa.

1838. Drymoica Pallidda.

B. M.

Drymoica pallida, Smith, Ill. Zool. S. Afr. pl. 72, f. 2. Drymoeca pallida, $C a b$. Sylvia limonella, Licht.

S. Africa.

1839. Drymoica affinis.

B. $M$.

Drymoica affinis, Smith, Ill. Zool. S. Afr. pl. 77, f. 1 .

S. Africa.

1840. Drymoica mxstacea.

Prinia mystacea, Rüpp. Drymoica mystacea, Rüpp. Syst. Uebers. t. 10.

N.-E. Africa.

1841. Drymoica superciliosa.

Drymoica superciliosa, Swains. B. of W, Afr. ii. pl. 2.

W. Africa.

1842. Drymoica subfiava.

Motacilla subflava, Gm. $\quad P l$. enl. 584, f.2, 3. Drymoica subflara, G. R. Gr. Sylvia subflava, Lath. S. flavicans, Vieill. Curruca subflava, Cuv. Malurus subtlarus, Steph. Levaill. Ois. de l'Afr, t. 127.

S. and W. Africa.

1843. Drymoica gracmis.

Malurus gracilis, Rüpp. Zool. Atlas, t. 2, f. b. $\quad P l . c o l .466$, f. 1. Drymœea gracilis, $\mathrm{Cab}$. Sylvia gracilis, Licht. S. textrix, Aud. Descr. de l'Egypte, Ois. t. 5, f. 4. Cisticola gracilis,

Less. Prinia vel Drymoica gracilis, Rüpp.

N. Africa (Egypt). 
1844. Drymoica coriphea.

Drymoica coriphea, G. R. Gr. Levaill. Ois. de l'Afr. t. 120. Sylvia coryphæus, Vieill. Arundinaceus coryphæus, Less. Thamnobia coryphæa, $\mathrm{Cab}$.

S. Africa.

1845. Drymoica diophrys.

Motacilla diophrys, Shaw, Nat. Misc. pl. 973. Levaill. Ois. de l'Afr. t. 128. Drymoica diophrys, G. R. Gr. Malurus diophrys, Steph.

S. Africa.

1846. Drymotca pectoralis. B. M.

Drymoica pectoralis, Smith, Ill. Zool. S. Afr. pl. 75, f. 2.

S. Africa.

1847. Drymorca thoracica.

Motacilla thoracica, Shaw, Nat. Misc. pl.969. Gen.Zool. х. pl. p. 562. Drymoica thoracica, G. R. Gr. Apalis thoracica, Sw. Zool. Illustr. n. s. pl. 119. Sylvia gutturalis, Boie. Regulus? thoracica, Steph. Levaill. Ois. de l'Afr. t. 123.

W. and S. Africa.

1848. Drymoica ocularius.

B. M.

Drymoica ocularius, Smith, Ill. Zool.S. Afr. pl.75, f. 1.

S. Africa.

1849. Drymoica aberRans.

B. $M$.

Drymoica aberrans, Smith, Ill. Zool. S. Afr. pl. 78.

S. Africa.

1850. Drtaroica Smithit.

B. M. Drymoica Smithi, Pr. B. D. ruficapilla, Smith, Ill. Zool. S. Afr. pl. 73, f. 1. Sylvia fulvicapilla, Vieill.? Smith.

S. Africa. 
1851. Drymoica fulvicapilla.

Sylvia fulvicapilla, Vieill. Lenaill. Ois. de l'Afr. t. 124. Drymoica fulvicapilla, G. R. Gr.

S. Africa.

1852. Drymoica NigRirostris.

B. $\mathrm{M}$.

Drymoica ruficapilla aut D. nigrirostris, $F r$.

W. Africa (River Nun).

1853. Drymoica Levaillantir.

B. M.

Drymoica Levaillantii, Smith, Ill. Zool. S. Afr. pl.73, f. 2. Malurus tiniens, Licht. Edon tiniens, G.R. Gr. Cisticola Levaillantii, $\mathrm{Cab}$.

S. Africa.

1854. Drymoica lugubris.

Sylvia (Cisticola) lugubris, Rüpp. Drymoica lugubris, Rüpp. Syst. Uebers, t. 11.

N.-E. Africa.

1855. DRYMOICA ERYTHROGENYS.

Drymoica erythrogenis, Rüpp. Sylvia (Cisticola) erythrogenis, Rüpp. Syst. Uebers. t. 12.

N.-E. Africa.

1856. Drymoica robusta

Drymoica robusta, Rüpp. Syst. Uebers. t. 13.

N.-E. Africa.

1857. Drymoica rUficeps.

Malurus ruficeps, Rüpp. Zool. Allas, t. 36, f. a. Prinia (?) vel

Drymoica ruficeps, $R$ upp.

N.-E. Africa.

1858. Drymoica SUBRUficapilla.

B. $\mathbf{M}$.

Drymoica subruficapilla, Smith, Ill. Zool. S. Afr. pl. 76, f. 2 .

S. Africa. 
1859. Drymoica undata.

Motacilla undata, Gm. Pl. enl. 582, f. 2. Sylvia undata, Lath. Drymoica undata, G. R. Gr.

S. Africa.

1860. Drimoica Strangei.

Drymoica Strangei, Fr.

B. M.

W. Africa (Accra).

1861. Drymoica mentalis.

B. $M$.

Drymoica mentalis, Fr. Jard. Contr. of Orn. pl.

W. Africa (Accra).

1862. Drymoica lateralis.

Drymoica lateralis, $F r$.

B. M.

W. Africa (Cape Palmas).

1863. Drymoica RUfifrons.

Prinia rufifrons, Rïpp. Faun. Abyss. t. 41, f. 1. Drymoica rufifrons, Rüpp.

N.-E. Africa.

1864. Drymoich RUFA.

B. M.

Drymoica rufa, $F r$.

W. Africa (River Quorra).

1865. Drymoica erythroptera.

Drymoica erythroptera, Jard. Contr. of Ornith. 1849, pl.

1866. Drpmoica natalensis.

B. M.

Drymoica natalensis, Smith, Ill. Zool. S. Afr. pl. 80.

S. Africa.

1867. Drymoica chiniana.

B. M.

Drymoica chiniana, Smith, Ill. Zool. S. Afr. pl. 79.

S. Africa. 
1868. Drymoica cherina.

B. M.

Drymoica cherina, Smith, Ill. Zool. S. Afr. pl. 77, f. 2.

S. Africa.

1869. Drymoica terRestris.

B. M.

Drymoica terrestris, Smith, Ill. Zool.S.Afr. pl. 74, f. 2.

S. Africa.

1870. DRYMOICA UROPYGIALIS.

B. M.

Drymoica uropygialis, $F r$.

W. Africa (Accra).

1871. Drymoica inquieta.

B. $M$.

Malurus inquietus, Rüpp. Zool. Atlas, t. 36, f. b. Prinia (?) vel Drymoica inquieta, Rüpp.

Arabia.

1872. DRymorca Clamans.

B. M.

Malurus clamans, Rüpp. Pl. col. 466, f. 2. Prinia et Drymoica clamans, Rüpp. Zool. Atlas, t. 2, f. a. Curruca clamans, Cuv.

N.-E. Africa.

1873. Drymoica pulchella.

Malurus pulchellus, Rüpp. Zool. Atlas, t. 35, f. a. Prinia vel Drymoica pulchella, Rüpp.

N.-E. Africa.

1874. Drymoica stricklandi.

B. M.

Prinia Stricklandi, Pr. B. Prinia olivacea, Strickl.

Fernando Po.

1875. Drymoica RUFOGULARIS.

B. M.

Drymoica rufogularis, $F r$.

Fernando Po.

1876. DrTmoica subcinnamomea.

B. M.

Drymoica subcinnamomea, Smith, Ill. Zool. S. Afr.pl. 111, f. 1 .

S. Africa. 


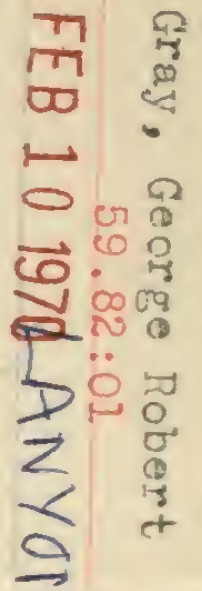




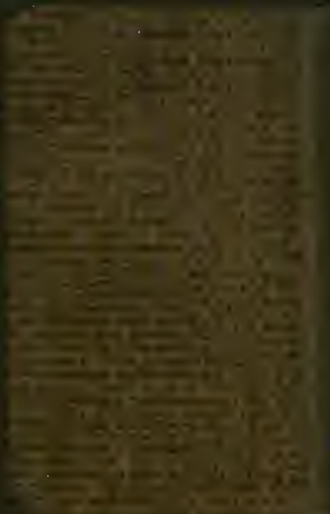

